\title{
Procura por Simetrias de Lie na Evolução do Código Genético
}

\section{Lígia Braggion}

Orientação: Prof. Dr. José Eduardo Martinho Hornos

Dissertação apresentada ao Instituto de Ciências Matemáticas de São Carlos - USP, como parte dos requisitos para a obtenção do título de Mestre em Ciências - Área de Ciências de Computação e Matemática Computacional. 
Aos meus pais,

Angelo e Dalzisa. 
Este trabalho teve o apoio financeiro da Fapesp. 


\section{Agradecimentos}

A todos que se fizeram presentes durante estes sete anos em São Carlos, em especial:

- ao Prof. Hornos, meu orientado e educador, pelo incentivo, pela paciência, compreensão e confiança.

- ao prof. Forger, pela grande ajuda.

- aos meus pais, para quem também dedico este trabalho, que sempre me incentivaram e me apoiaram, estando sempre ao meu lado quando precisei.

- ao João, Maria Helena, Denise e Edymara, pelo incentivo, apoio e atenção.

- às grandes amigas de "festa ", Claudia, Silvia Arantes e Silvia Marinelli, pelas boas noitadas.

- as amigas de toda hora, Claudia Pio, Iaio e Cris, "na saúde e na doença, na alegria e na tristeza".

- a todos os amigos e professores de graduação e pós, em especial ao mestre João Peneireiro (professor e amigo).

- aos colegas de grupo, Mauro, Esmerindo, Jean, Trovão, Zésinho, Fernandinho, Antônio Sérgio e Marcio.

- finalmente ao Vaguinho ("Xuxinho"), pela paciência, companheirismo e pelo amor. 


\section{Índice}

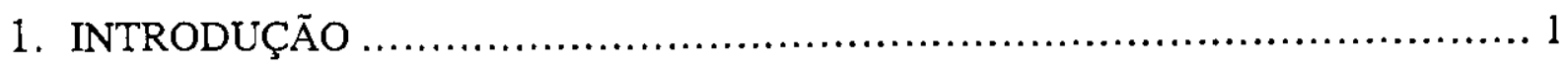

2. O CÓDIGO GENÉTICO................................................. 3

2.1 Proteínas e Aminoácidos ......................................................

2.2 DNA, RNA e Bases Fundamentais ............................................4

2.3 Processo de Síntese de Proteínas.............................................6

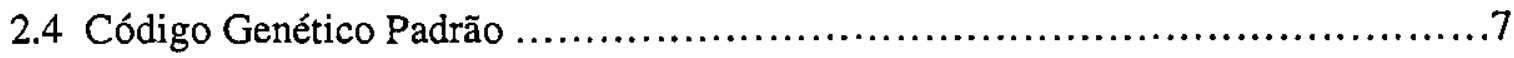

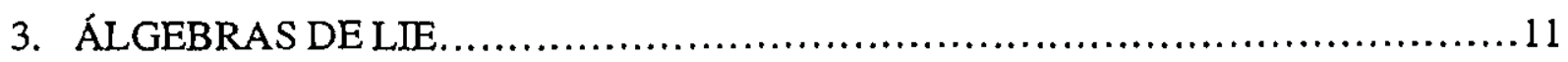

3.1 Introdução às Álgebras de Lie................................................1

3.2 Álgebras de Lie Clássicas................................................ 13

3.3 Álgebras de Lie e Subálgebras............................................18

3.4 Ideais e Ideais Próprios................................................. 18

3.5 Representações Adjuntas das Álgebras de Lie................................ 18

3.6 Soma Direta e Soma Semi-Direta........................................... 20

3.7 Álgebras de Lie Solúveis e Nilpotentes....................................... 20

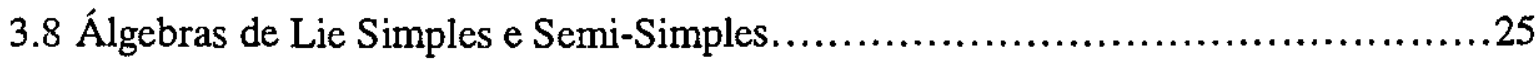

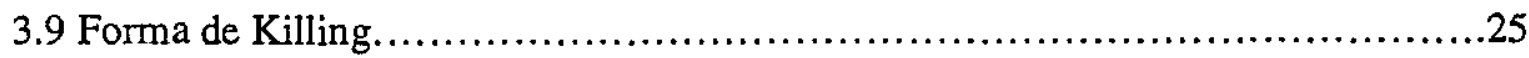

3.10 Os Operadores de Casimir.................................................. 30

3.11 Sistema de Raízes......................................................... 33

3.11.1 Forma Canônica de Cartan Wey....................................... 33

3.11.2 A Matriz de Cartan....................................................... 38

3.11.3 Forma Canônica de Chevalley ........................................ 39

3.12 Representações das Álgebras de Lie........................................40

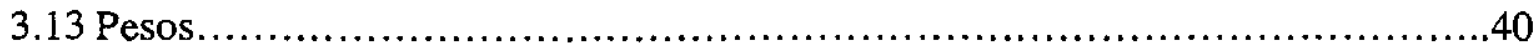

4. DETERMINÇÃO DAS CADEIAS SOBREVIVENTES ...........................43

4.1 Representações do Espaço dos Códons........................................ 43

4.2 Quebra de Simetria por Cadeias de Subálgebras................................51

4.3 Quebra da Simetria Primordial para Simetria su(2).............................55

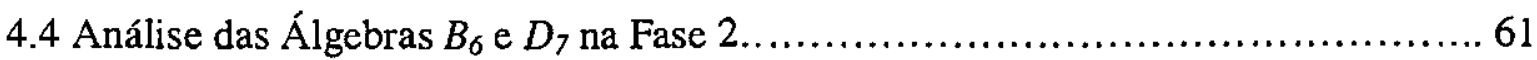




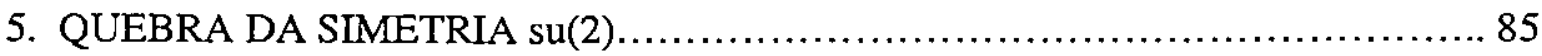

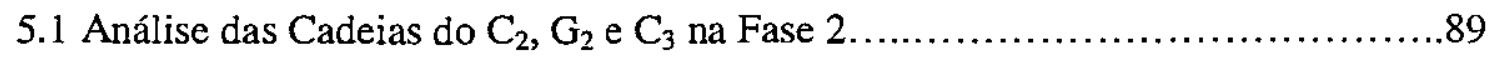

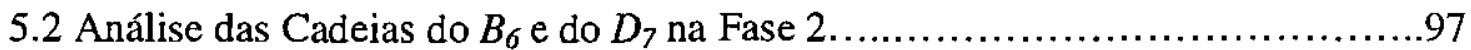

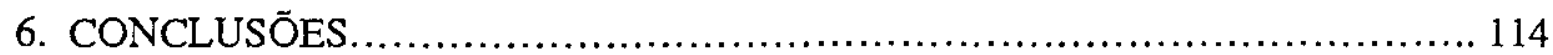

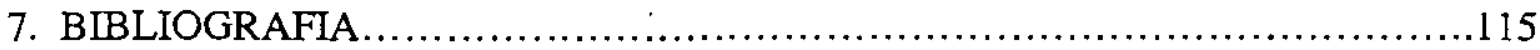




\section{Lista de figuras}

Figura 1: Estrutura dos aminoácidos.............................................

Figura 2: Estrutura molecular das bases fundamentais do DNA . .........................4

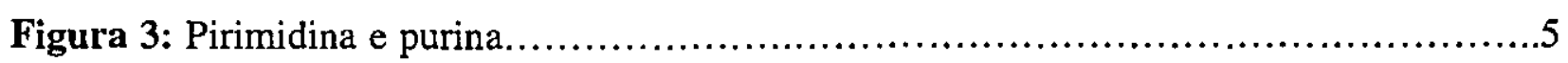

Figura 4: Fluxo de informação do DNA para proteínas ..................................

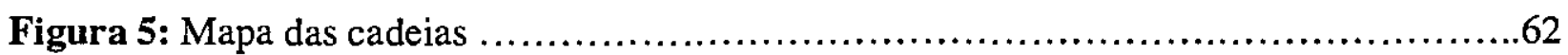




\section{Lista de Tabelas}

Tabela 1: $O$ código genético

8

Tabela 2-a ${ }_{1}$ : Pesos máximos e dimensões das representações irredutíveis das álgebras de Lie clássicas $A_{r}$ de rank baixo 45

Tabela 2-a 2 : Pesos máximos e dimensões das representações irredutíveis das álgebras de Lie clássicas $A_{r}$ de rank baixo.

Tabela 2-b: Pesos máximos e dimensões das representações irredutíveis das álgebras de Lie clássicas $B_{r}$ de rank baixo

Tabela 2-c: Pesos máximos e dimensões das representaçōes irredutíveis das álgebras de Lie clássicas $C_{r}$ de rank baixo.

Tabela 2-d: Pesos máximos e dimensões das representações irredutíveis das álgebras de Lie clássicas $D_{r}$ de rank baixo

Tabela 2-e: Pesos máximos e dimensões das representações irredutíveis das álgebras de Lie excepcionais $E_{6}, E_{7}, E_{8}, F_{4}$ e $G_{2}$, de rank baixo .50

Tabela 3: Representações do tipo códon de álgebras de Lie simples .51

Tabela 4: Subálgebras maximais semi-simples das álgebras de Lie simples .52

Tabela 5: Dimensões e multiplicidades do código genético padrão

Tabela 6: Quebra da representação do tipo códon do su(3) nas cadeias não-sobreviventes su(3)

$\supset \mathrm{su}(2)$ e su(3) つ so(3)

Tabela 7: Quebra da representação do tipo códon do sp(4) na cadeia sobrevivente

$\mathrm{sp}(4) \supset \mathrm{su}(2) \oplus \mathrm{su}(2)$, e na cadeia não-sobrevivente $\mathrm{sp}(4) \supset \mathrm{su}(2)$

Tabela 8: Quebra da representação do tipo códon do $G_{2}$ na cadeia sobrevivente $G_{2} \supset \mathrm{su}(2) \oplus \mathrm{su}(2)$ e nas cadeias não-sobrevivente $G_{2} \supset \mathrm{su}(3)$ e $G_{2} \supset \mathrm{su}(2)$ .58

Tabela 9-a: Quebra da representação do tipo códon do sp(6) nas cadeias não-sobreviventes $\mathrm{sp}(6) \supset \mathrm{su}(2)$ e $\operatorname{sp}(6) \supset \mathrm{su}(2) \oplus \mathrm{su}(2)$

Tabela 9-b: Quebra da representação do tipo códon do $\mathrm{sp}(6)$ nas cadeias sobreviventes $\mathrm{sp}(6) \supset \mathrm{sp}(4) \oplus \mathrm{su}(2) \supset \mathrm{su}(2) \oplus \mathrm{su}(2) \oplus \mathrm{su}(2)$ e $\mathrm{sp}(6) \supset \mathrm{sp}(4) \oplus \mathrm{su}(2) \supset \mathrm{su}(2) \oplus \mathrm{su}(2) \ldots 60$ Tabela 10: Quebra da representação tipo códon do $\mathrm{sp}(4)$ na cadeia $\mathrm{sp}(4) \supset \mathrm{su}(2) \oplus \mathrm{su}(2) \supset \mathrm{o}(2) \oplus \mathrm{su}(2) \supset \mathrm{so}(2) \oplus \mathrm{su}(2)$ 90

Tabela 11a: Quebra da representação tipo códon do $G_{2}$ na cadeia 
$\mathrm{G}_{2} \supset \mathrm{su}(2) \oplus \mathrm{su}(2) \supset \mathrm{o}(2) \oplus \mathrm{su}(2) \supset \mathrm{o}(2) \oplus \mathrm{o}(2)$

Tabela 11b: Quebra da representação tipo códon do $G_{2}$ na cadeia

$\mathrm{G}_{2} \supset \mathrm{su}(2) \oplus \mathrm{su}(2) \supset \mathrm{su}(2) \oplus \mathrm{o}(2) \supset \mathrm{su}(2) \oplus \mathrm{so}(2)$

Tabela 12: Quebra da representação tipo códon do sp(6) na cadeia

$\mathrm{sp}(6) \supset \mathrm{sp}(4) \oplus \mathrm{su}(2) \supset \mathrm{su}(2) \oplus \mathrm{su}(2) \supset \mathrm{o}(2) \oplus \mathrm{su}(2)$.

Tabela 13: Quebra da representação tipo códon do sp(6) na cadeia

$\mathrm{sp}(6) \supset \mathrm{sp}(4) \oplus \mathrm{su}(2) \supset \mathrm{su}(2) \oplus \mathrm{su}(2) \oplus \mathrm{su}(2) \supset \mathrm{su}(2) \oplus \mathrm{o}(2) \oplus \mathrm{su}(2)$

$\supset \mathrm{su}(2) \oplus \mathrm{o}(2) \oplus \mathrm{so}(2)$

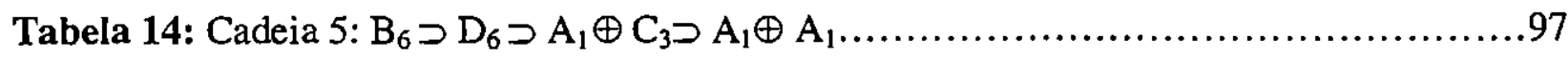

Tabela 14a: Quebra da cadeia 5 em $L_{2, z}^{2}$ (não-sobrevivente) ...........................98

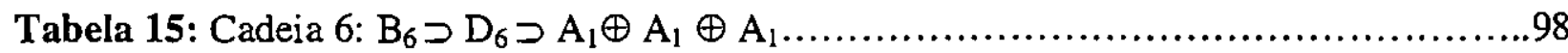

Tabela 15a: Quebra da cadeia 6, primeiro em $L_{1,2}^{2}$ e depois em $L_{1,2}$ (não-sobrevivente)........99

Tabela 15b: Quebra da cadeia 6, primeiro em $L_{1, z}^{2}$ e depois em $L_{2, z}^{2}$ (não-sobrevivente).....100

Tabela 16: Cadeia 7: $D_{7} \supset B_{3} \oplus B_{3} \supset B_{3} \oplus G_{2} \supset G_{2} \oplus G_{2} \supset G_{2} \oplus A_{1} \supset A_{1} \oplus A_{1} \oplus A_{1} \ldots \ldots . .100$

Tabela 16a: Quebra da cadeia 7, em $L_{3,2}^{2}$ (não-sobrevivente)............................ 101

Tabela 16b: Quebra da cadeia 7, primeiro em $L_{1,2}$ e depois em $L_{3, z}^{2}$ (não-sobrevivente).....102

Tabela 17: Cadeia 8: $D_{7} \supset A_{3} \oplus D_{4} \supset A_{3} \oplus A_{1} \oplus C_{2} \supset A_{3} \oplus A_{1} \oplus A_{1} \supset A_{2} \oplus A_{1} \oplus A_{1}$

$\supset A_{1} \oplus A_{1} \oplus A_{1}$ 102

Tabela 17a: Quebra da cadeia 8 primeiro em $L_{2, z}^{2}$ e depois em $L_{3, z}^{2}$ e $L_{3, z}$ (não-sobrevivente)

Tabela 17b: Quebra da cadeia 8 em $L_{2 . z}$ (não-sobrevivente) ........................... 104

Tabela 18: Cadeia $9: D_{7} \supset A_{1} \oplus A_{1} \oplus D_{5} \supset A_{1} \oplus A_{1} \oplus C_{2} \supset A_{1} \oplus A_{1} \oplus A_{1} \oplus A_{1} \ldots \ldots \ldots . . .104$

Tabela 18a: Quebra da cadeia 9 em $L_{3,2}^{2}$ e depois em $L_{1, z}$ (não-sobrevivente)................105

Tabela 18b: Quebra da cadeia 9 em $L_{3, z}$ e depois em $L_{1, z}$ (não-sobrevivente).................105

Tabela 19: Cadeia 10: $D_{7} \supset A_{1} \oplus A_{1} \oplus D_{5} \supset A_{1} \oplus A_{1} \oplus A_{4} \supset A_{1} \oplus A_{1} \oplus C_{2} \supset A_{1} \oplus A_{1} \oplus A_{1} \oplus A_{1}$. . .106

Tabela 19a: Quebra da cadeia 10 em $L_{1, z}$ e em $L_{2, z}$, simultaneamente (não-sobrevivente)...107

Tabela 20: Cadeia 11: $D_{7} \supset A_{1} \oplus A_{1} \oplus D_{5} \supset A_{1} \oplus A_{1} \oplus A_{4} \supset A_{1} \oplus A_{1} \oplus A_{1} \oplus A_{2} \supset A_{1} \oplus A_{1} \oplus A_{1} \oplus A_{1}$. 
Tabela 20a: Quebra da cadeia 11 em $L_{3 . \mathrm{z}}$ e $L_{3, \mathrm{z}}$ e $L_{1, \mathrm{z}}$ simultaneamente (não-sobrevivente)..108 Tabela 21: Cadeia 12: $D_{7} \supset A_{3} \oplus D_{4} \supset A_{3} \oplus A_{1} \oplus C_{2} \supset A_{3} \oplus A_{1} \oplus A_{1} \oplus A_{1}$

$\supset A_{2} \oplus A_{1} \oplus A_{1} \oplus A_{1} \supset A_{1} \oplus A_{1} \oplus A_{1} \oplus A_{1}$

Tabela 22: Cadeia 13: $D_{7} \supset B_{3} \oplus B_{3} \supset B_{3} \oplus G_{2} \supset G_{2} \oplus G_{2} \supset G_{2} \oplus A_{1} \oplus A_{1} \supset A_{1} \oplus A_{1} \oplus A_{1} \oplus A_{1}$

Tabela 22a: Quebra da cadeia $13 \mathrm{em}\left(L_{1.2}, L_{2.2}^{2}, L_{3 . \mathrm{z}}\right)$ (não-sobrevivente).

Tabela 23: Cadeia 14: $D_{7} \supset B_{3} \oplus B_{3} \supset B_{3} \oplus A_{1} \oplus A_{1} \oplus A_{1} \supset G_{2} \oplus A_{1} \oplus A_{1} \oplus A_{1}$

$\supset \mathrm{A}_{1} \oplus \mathrm{A}_{1} \oplus \mathrm{A}_{1} \oplus \mathrm{A}_{1} \oplus \mathrm{A}_{1}$

Tabela 23a: Quebra da cadeia $14 \mathrm{em} L_{1, \mathrm{z}}$ e depois em $L_{5, \mathrm{z}}$ (não-sobrevivente). 111

Tabela 24 : Cadeia $15: D_{7} \supset A_{3} \oplus D_{4} \supset A_{3} \oplus A_{1} \oplus A_{1} \oplus A_{1} \oplus A_{1} \supset A_{2} \oplus A_{1} \oplus A_{1} \oplus A_{1} \oplus A_{1}$

$\supset \mathrm{A}_{1} \oplus \mathrm{A}_{1} \oplus \mathrm{A}_{1} \oplus \mathrm{A}_{1} \oplus \mathrm{A}_{1}$

Tabela 24a: Quebra da cadeia $15 \mathrm{em}\left(L_{2 . \mathrm{z}}, L_{3 . \mathrm{z}}\right)$ (não-sobrevivente). 


\section{Resumo}

Apresentamos nesta dissertação, uma análise do modelo algébrico para o código genético proposto por Hornos e Hornos, o qual procura explicar as degenerescências do código genético como o resultado de uma sequência de quebras de simetrias que tenha ocorrido durante a evolução. Fizemos uma procura sistematizada por possíveis simetrias no código genético, através de uma análise minuciosa de todas as álgebras de Lie simples que possuem uma representação irredutível de dimensão 64 e de todas as suas cadeias de subálgebras maximais. Os resultados confirmam e sistematizam as conclusões de Hornos e Hornos e Forger et all. 


\section{Abstract}

We introduce in this dissertation an analysis of the algebraic approach to the genetic code proposed by Hornos and Hornos, which aims at explaining the degeneracies in the genetic code as the result of a sequence of symmetry breakings that have occurred during the evolution. We making a systematic search for symmetries possible in the genetic code, through a detailed analysis of the all simple Lie algebras that possess an irreducible representation of 64 dimension and of every possible chains of maximal subalgebras. The results confirm and systematize the Hornos and Hornos and Forger et all conclusion. 


\section{Capítulo 1}

\section{Introdução}

A descoberta da estrutura molecular do DNA feita por Watson e Crick ${ }^{11}$ em 1953 foi um marco divisório na história da ciência. O DNA é conhecido desde 1869 e foi reconhecido como o carregador de informação genética em 1944 mas suas bases biológicas, estabilidade e capacidade de reprodução continuaram um mistério. Com a descoberta do modelo de dupla hélice, geneticistas criaram uma nova área, a biologia molecular.

O modelo da dupla hélice de Watson e Crick deixou claro que a informação genética é armazenada no DNA na forma de sequiências de nucleotídeos, mas ele não fornece nenhuma sugestão de como a informação é exatamente codificada em uma seqüência, nem como ela se expressa para exercer suas funções biológicas específicas. Teoricamente esta questão consiste no problema de decifrar o código genético. Uma das primeiras tarefas da biologia molecular foi procurar como o código genético conduziu a síntese de proteínas, a mais complicada classe de moléculas que aparecem em organismos vivos.

O primeiro passo nessa direção foi a descoberta de Crick $^{12}$ em 1960, que o código genético é um código triplo, em outras palavras, que sua unidade elementar de informação, chamada códon, é uma sequiência de três bases nucleicas cada qual representando um único aminoácido. Logo em seguida trabalhos experimentais ${ }^{13-16}$ levaram a uma completa classificação da correspondência entre códons e aminoácidos, totalmente esclarecida em $1966 \mathrm{e}$ reuniu-os em uma tabela padrão a qual pode ser encontrada em qualquer livro texto sobre bioquímica ou genética, neste trabalho ela está reproduzida como tabela 1.

Uma característica marcante da tabela do código genético é sua degenerescência. Esta degenerescência é inevitável e se deve ao fato de que existem 64 códons enquanto que somente 20 aminoácidos são encontrados em todos os organismos vivos. Experiências acumuladas em 
mais de 50 anos de pesquisa em física tem revelado que a degenerescência é uma consequiência de simetria.

Recentemente foi proposto um modelo matemático para a evolução do código genético universal baseado na teoria das álgebras de Lie e no conceito de quebra espontânea de simetrias $^{1-3}$. A teoria foi construída a partir de uma procura sistemática de simetrias que se ajustassem às degenerescência do código genético. Todas as álgebras simples classificadas foram investigadas com a exigência da existência de uma representação irredutível de dimensão 64 associada aos códons que codificam os 20 aminoácidos fundamentais e o códon de terminação. Essa investigação foi feita realizando as quebras de tais simetrias através de cadeias de subálgebras de maneira que cada representação irredutível da subálgebra anterior se decompõe em uma família de representações irredutíveis da subálgebra seguinte. Os resultados indicavam a inexistência de uma álgebra de Lie simples que atravês de uma cadeia de subálgebras apropriada reproduzisse exatamente a degenerescência do código genético. No entanto essa investigação mostrou que somente a álgebra simplética $\mathrm{sp}(6)$ em sua cadeia maximal que contêm a álgebra $\operatorname{sp}(4) \oplus \operatorname{su}(2)$ que por sua vez se decompõe em $\operatorname{su}(2) \oplus \operatorname{su}(2) \oplus$ $\mathrm{su}(2)$ consegue reproduzir corretamente as degenerescências do código universal, se no último estágio do processo de quebra, ocorrer uma interrupção parcial, ou seja, um congelamento apropriado.

No entanto, este modelo, o qual introduziu as técnicas algébricas em genética, apresenta os resultados da procura por simetrias no código genético sem mostrar os detalhes que levaram a essa conclusão. Este trabalho tem como objetivo refazer toda essa análise de forma sistemática, mostrando e explicando os argumentos e expondo os resultados em sua íntegra, de maneira que a comunidade cientifica possa ter acesso aos detalhes técnicos do processo.

Essa dissertação é organizada como se segue. No capítulo 2 apresentamos uma breve discussão as principais características do código genético. O capítulo 3 é reservado para as álgebras de Lie. A determinação das cadeias sobreviventes candidatas a reproduzirem as degenerescências do código genético é feita no capítulo 4. No capítulo 5, exibimos a quebra da simetria $\mathrm{su}(2)$ nas cadeias sobreviventes do capitulo 4. Finalmente apresentamos as conclusões no capítulo 6 e a bibliografia no capítulo 7 . 


\section{Capítulo 2}

\section{O Código Genético}

Este capítulo exibe algumas noções de genética, apresentando uma breve discussão sobre as principais características do código genético.

\subsection{Proteínas e Aminoácidos}

A família dos aminoácidos contêm mais que 200 variedades mas somente 20 aminoácidos fundamentais aparecem nas proteínas em todas formas de vida independentemente das espécies. Algumas vezes um aminoácido não usual aparece em uma proteína, mas ele é uma modificação de algum dos aminoácidos fundamentais que ocorre depois da proteína ser construída na célula .

A estrutura molecular dos aminoácidos é muito simples. Consiste de um grupo comum em que um carbono $\alpha$ forma ligações covalentes com um grupo amino $\left(\mathrm{NH}_{2}\right)$, uma carboxila $(\mathrm{COOH})$, um hidrogênio e um radical que especifica o aminoácido em particular. A estrutura dos aminoácidos é mostrada na figural.<smiles>[R]C(N[TlH])C(=O)O</smiles>

Figura 1: Estrutura dos aminoácidos. 
$O$ radical pode variar do simples caso $R=H$, para glicina, para estruturas mais complexas as quais podem envolver anéis aromáticos ou cadeias alifáticas. Varias propriedades físico-químicas dos aminoácidos tem sido tabuladas, algumas importantes do ponto de vista genético. E o caso das polaridades que desempenham um importante papel na relação entre proteína e água.

Proteínas são construídas como uma cadeia de aminoácidos através de ligações peptídicas na qual o carbono carboxila forma uma ligação covalente com o hidrogênio do aminoácido ao lado. Depois a proteína é agrupada e se dobra tomando uma forma muito complicada, chamada estrutura terciária.

\subsection{DNA, RNA e Bases Fundamentais}

Em todas as formas de vida na terra, a informação genética é relatada em dois polímeros chamados de DNA (ácido dexórribonucleico) e RNA (ácido ribonucleico), compostos por estruturas unitárias chamadas nucleotídeos. Cada uma dessas moléculas é feita de uma molécula de açúcar (dexórribose no DNA, ribose no RNA), um grupo fosfato e uma base nucleica que é escolhida dentre quatro bases fundamentais a Adenina (A), Citosina (C), Guanina (G) e Timina (T) no DNA ou Uracila (U) no RNA. No mecanismo responsável pela sustentação da vida e, em particular, a síntese de proteínas, o DNA é o material genético primário, enquanto que o RNA é o material genético secundário.

A estrutura molecular das bases fundamentais do DNA é mostrada na figura 2, onde Timina, Citosina e Uracila são derivadas de uma molécula mãe, a pirimidina, Adenina e Guanina são derivadas da purina. Na figura 3 mostramos as moléculas mães, purina e pirimidina.<smiles>Nc1ncnc2[nH]cnc12</smiles>

Adenina<smiles>Nc1nc2[nH]cnc2c(=O)[nH]1</smiles>

Guanina<smiles>Nc1cc[nH]c(=O)n1</smiles>

Citosina<smiles>O=c1cc[nH]c(=O)[nH]1</smiles>

Uracila

Figura 2: Estrutura molecular das bases fundamentais do DNA. 
<smiles></smiles>

Pirimidina<smiles>c1nc2cnc[nH]c-2n1</smiles>

Purina

Figura 3: Pirimidina e purina.

As moléculas de DNA são conhecidas desde 1869, mas elas não foram reconhecidas como carregadoras de informação hereditária até 1944 . Antes métodos cromatográficos seguros foram avaliados e acreditou-se que a composição das 4 bases fundamentais encontradas no DNA eram as mesmas em todas formas de vida. Entre 1944 e 1953 foi feita por Chargaff e colaboradores uma análise quantitativa da composição das quatros bases e descobriram que a composição das bases era a mesma em diferentes tecidos da mesma espécie mas diferia de espécie para espécie. Outro resultado importante dessa pesquisa, conhecido como regra de Chargaff, foi o fato de que a razão do conteúdo de $\mathrm{A}$ para o conteúdo de $\mathrm{T}$ e do conteúdo de $\mathrm{C}$ para $G$ é sempre um, independente da espécie.

Análises de difração de raios- $x$ de elementos acessíveis mostrou que o DNA é uma molécula fina e longa com dois espaçamentos regulares ao longo do eixo do filamento. Durante este período também foi estabelecida as dimensōes das bases purinas e pirimidinas, a qual faz a distinção estereoquímica das bases. Entretanto, nenhum dos modelos para o DNA propostos até 1953 proporcionou uma explicação precisa parà o mecanismo da replicação, a favor da estabilidade ou a favor da regra de Chargaff do conteúdo de base.

A questão da replicação precisa, estabilidade e base completa não foi respondida pelos modelos para o DNA proposto até 1953. O modelo de Watson-Crick para estruturas tridimensionais de DNA não somente expõe todas as propriedades físicas e químicas encontradas em DNA mas também propõe um mecanismo pelo o qual a informação genética poderia ser replicada com precisão.

O modelo de Watson-Crick propôs que o DNA é uma estrutura de dupla hélice com duas hélices de mão direita enroladas paralelamente. Cada hélice é composta por uma longa seqüência de nucleotídeos, que são estruturas unitárias compostas por uma base fundamental, 
um açúcar (desoxirribose) e um grupo fosfato. A dupla hélice aparece através de pontes de hidrogênio ligando as bases nucleicas de uma fita correspondendo com as bases de outra fita de acordo com as regras emparelhamento de Watson-Crick, ou seja, A emparelha somente com $\mathrm{T}$ e C emparelha somente com G.

O modelo de dupla hélice de Watson e Crick, não explica somente a física básica e as propriedades químicas do DNA mas também proporciona um mecanismo pelo qual a informação genética pode ser replicada com grande precisão. Por exemplo as regras do conteúdo de bases de Chargaff é uma consequiência direta do emparelhamento de WatsonCrick. Além disso a estrutura de dupla hélice reforça a estabilidade do DNA porque as duas fitas podem ser separadas somente desenrolando-as completamente e quebrando cada ponte de hidrogênio (o que requer uma energia da ordem de $0.1 \mathrm{eV}$ por ponte). O processo de replicação precisa, necessita da continuidade genética, células mães e células filhas feita possivelmente pela replicação semi-conservativa. A existência de muitas enzimas específicas (DNA polimerases), junto com um número de outras moléculas regulares torna possível o desenrolamento e duplicação das fitas com fidelidade. Outras enzimas são responsáveis pela recombinação genética das duas fitas.

No RNA, existe somente uma fita, mas o emparelhamento das bases através de pontes de hidrogênio ocorre porque a fita pode dobrar para trás sobre ela mesma e formar seguimentos helicoidais duplos de bases nucleicas emparelhadas interrompidas pelos loops com bases nucleicas desemparelhadas. Este tipo de estrutura é comum em todas formas de RNA, isto é, para o RNA mensageiro (mRNA), para o RNA transportador (tRNA) e para o RNA ribossômico (rRNA), os quais desempenham diferentes funções no processo de síntese de proteínas, que veremos no próximo item deste capítulo.

\subsection{Processo de Síntese de Proteínas}

O processo de síntese de proteínas implica no fluxo de informação do DNA, que situase nos núcleos das células eucariontes, para os ribossomos que são organelas citoplasmátcas onde ocorre a síntese de proteínas. Em 1961 F. Jacob e J. Monod propuseram um mecanismo de transcrição e tradução destas informações postulando a existência do RNA mensageiro. Esquematicamente nós mostramos o fluxo de informação do DNA para proteínas na figura 4. 
DNA $\rightarrow$ RNA $\rightarrow$ proteína

transcrição traduçāo

Figura 4: Fluxo de informação do DNA para proteínas.

O primeiro passo na síntese de proteínas é a transcrição, durante a qual a informação da sequiência de aminoácidos de uma proteína em particular, contida no DNA, é copiada por uma molécula mRNA, de acordo com as regras da dualidade de Watson-Crick, daí esta molécula de mRNA leva a informação para os ribossomos.

Uma molécula de mRNA contêm a sequiência de uma proteína específica para ser sintetizada no ribossomo. Esta molécula de mRNA é muito menor que a molécula de DNA que é copiada, mas mesmo assim pode carregar milhões de nucleotídeos.

O segundo passo na síntese de proteínas é a tradução, a qual ocorre nos ribossomos. O processo de tradução depende crucialmente do tRNA a qual carrega um aminoácido específico do citoplasma para os ribossomos e simultaneamente lê a informação contida em uma seqüência de três bases do mRNA escolhidas dentre as quatro bases fundamentais(Uracila, Citosina, Guanina e Adenina), chamada códon. O sítio de reconhecimento do molde no tRNA é uma seqüência de três bases chamada anticódon a qual reconhece o códon no mRNA representando o aminoácido correto.

O processo de tradução é iniciado quando o ribossomo reconhece o sinal de começo exibido pelo mRNA e se acopla na molécula de mRNA, começando a deslizar ao longo dela, daŕ, sempre que uma molécula de tRNA apropriada, carregada com um aminoácido e exibindo o correto anticódon para acoplar com o códon exibido no mRNA, entra o ribossomo, o aminoácido será liberado e ligado a cadeia de aminoácidos já existente através da formação de uma nova ponte peptidica. Posteriormente, a molécula de tRNA descarregada também será liberada, e o ribossomo se move para o próximo códon sobre o mRNA, para repetir o processo, até encontrar um sinal de fim e a molécula mRNA ser liberada do ribossomo completamente.

\subsection{O Código Genético Padrão}


O código genético, totalmente decifrado em 1966, é um mapa que associa a cada tripleto de base (códon), um aminoácido. As bases em cada códon são selecionadas dentre as quatro bases fundamentais, Uracila(U), Citosina(C), Guanina(G), e Adenina(A) formando 64 possíveis códons. Somente 20 aminoácidos aparecem em todas as proteínas e 61 trincas correspondem a estes aminoácidos, enquanto três códons indicam o final do processo de formação do peptídeo. Os 64 códons são mostrados na tabela 1 junto com a abreviação dos aminoácidos associados a eles.

\begin{tabular}{|c|c|c|c|c|c|}
\hline \multirow{2}{*}{$\begin{array}{l}\text { PRIMEIRA } \\
\text { POSIÇĀOO }\end{array}$} & \multicolumn{4}{|c|}{ SEGUNDA POSICCĀO } & \multirow{2}{*}{$\begin{array}{c}\text { TERCEIRA } \\
\text { POSIÇÃOO }\end{array}$} \\
\hline & $U$ & $C$ & $A$ & $G$ & \\
\hline \multirow{4}{*}{$U$} & Fen & Ser & Tir & Cis & $\bar{U}$ \\
\hline & Fen & Ser & Tir & Cis & $c$ \\
\hline & Leu & Ser & Fim & Fim & $A$ \\
\hline & Leu & Ser & Fim & $\operatorname{Trp}$ & $G$ \\
\hline \multirow{4}{*}{$c$} & Leu & Pro & His & $\overline{A r g}$ & $\bar{U}$ \\
\hline & Leu & Pro & His & Arg & $c$ \\
\hline & Leu & Pro & $G l n$ & Arg & $A$ \\
\hline & Leu & Pro & Gln & Arg & $G$ \\
\hline \multirow{4}{*}{$A$} & lle & $T m$ & Asn & Ser & $\bar{U}$ \\
\hline & lle & $T m$ & Asn & Ser & $c$ \\
\hline & lle & $T_{m}$ & Lis & Arg & $A$ \\
\hline & Met & $T m$ & Lis & Arg & $G$ \\
\hline \multirow{4}{*}{$G$} & Val & $\overline{A l a}$ & $\overline{A s p}$ & $G l i$ & $\bar{U}$ \\
\hline & Val & Ala & Asp & Gli & $c$ \\
\hline & Val & Ala & Glu & Gli & $A$ \\
\hline & Val & Ala & Glu & Gli & $G$ \\
\hline
\end{tabular}

Tabela 1: O código genético.

Uma das propriedade importante do código genético é a sua degenerescência. O código é altamente degenerado, em outras palavras, muitos aminoácidos são determinados por mais de uma trinca, apenas o Triptofano e a Metionina são codificados por uma trinca, a Serina, Arginina, e Leocina estão associadas a 6 códons, a Isoleocina e o código terminal são tripletos e os demais aminoácidos tem degenerescência 2 ou 4.

Um significado biológico da extensa degenerescência do código genético é que a degenerescência diminui os efeitos deletérios das mutações. Se o código não fosse degenerado, 20 códons designariam os aminoácidoș, e 44 levariam ao término da cadeia, daí a probabilidade de mutação para o término da cadeia seria então muito mais alta com um código não- 
degenerado do que com o real, e mutações no termino da cadeia levam a proteínas inativas, enquanto que a substituição de um aminoácido por outro geralmente é menos prejudicial.

O código genético não é só altamente degenerado, mas a distribuição de códons representando o mesmo aminoácido mostra certas regularidades, tal como o significado de um códon ser dado na terceira base. Esta observação levou Crick a postular as " wobble hypothesis " de acordo com o emparelhamento entre a terceira base de um códon e a primeira base no correspondente anticódon não obedecendo necessariamente a regra rigorosa do emparelhamento W-C, ou seja, outros emparelhamentos são possíveis, como por exemplo o emparelhamento entre $\mathrm{G}$ e $\mathrm{U}$. Este permite um anticódon com $\mathrm{G}$ na primeira posição e simultaneamente reconhece códons com $\mathrm{U}$ e com $\mathrm{C}$ na terceira posição, tal que o aparecimento de um tRNA com um tal anticódon leva os dois códons a designarem o mesmo aminoácido. Logo depois foi encontrado anticódons que freqüentemente continham bases não usuais na primeira posição, tal como a inosina que permite regras de emparelhamento não convencionais na terceira base do códon. Existe agora uma extensa lista de regras "instáveis" e de correspondências códonanticódon em muitos tipos diferentes de organismos ${ }^{24}$.

A tentativa de explicar o código genético neste caminho, puramente na área biológica teve um ponto negativo, ela não explicava o fato de que apesar da riqueza de diversidade observada entre as espécies no processo de tradução, em particular considerando a grande variedade de anticódons e, mais geralmente de moléculas de tRNA, o código genético é quase universal. Na verdade o código genético padrão presente na tabela 1 durante a primeira década após sua descoberta acreditava-se ser estritamente universal, muito embora, agora sabemos que não é, os desvios encontrados em códigos não padrões são pequenos, em cada caso a modificação afeta somente um número pequeno de códons, aminoácidos designados e aplicados a uma classe de espécies muito restrita ou para códigos de organelas tais como mitocondrias e cloroplastos $^{24}$. Os argumentos normalmente usados por biologistas e geneticistas neste contesto é o primeiro apresentado por $\mathrm{Crick}^{25}$ quando formulou a famosa hipótese do "frozen accident" de acordo com o código genético, passando por uma fase primordial da evolução, foi em um certo estágio do congelamento em suas formas observadas, isto é, quando a maquinaria da síntese de proteínas em organismos foi tão complexa que, depois mudanças se tornaram letais. Universalmente seria uma consequiência do fato de que este congelamento ocorreu muito cedo na evolução até mesmo antes a bifurcação de formas de vidas em diferentes espécies. A análise em códigos não padrões e suas origens ${ }^{24}$ interpretaram com evidencia que o congelamento não 
é completo, alguns tipos de fusões podem ocasionalmente ocorrer. Uma simples afirmação que o código genético foi congelado em algum estágio de sua evolução não proporcionou nenhuma informação de como eram as leis que governavam a evoluçăo antes de ocorrer o congelamento. A hipótese do "frozen accident" estabelece que esta evolução primordial foi totalmente um problema de "oportunidade". Um argumento estatístico calculado por Bertman e Jungck ${ }^{26}$ mostra que o número de códigos genéticos possíveis é da ordem de $10^{71}$. Em vista desse argumento, é um desafio identificar as leis que vem governando a evolução do código genético.

O modelo algébrico para o código genético dirigi-se exatamente para esse problema, baseado na iđéia de que a degenerescência observada no código genético é uma reflexão de uma simetria primordial que no curso da evolução do código genético se deu em uma sequiência de passos. Uma das principais vantagens deste modelo é que as exigências de compatibilidade com alguma simetria reduz radicalmente o número de possibilidades mencionadas levando a uma possibilidade não insignificante para o código genético presente ser justamente o caminho. Neste sentido, o modelo algébrico é compatível com a idéia do congelamento.

Finalmente, nós gostaríamos de ressaltar que a decisão de aplicar técnicas em teoria de grupos para analisar a degenerescência do código genético está baseađa em experiências acumuladas em física, onde estas técnicas são úteis para analisar uma grande variedade de fenômenos estenđendo-se de física das partículas até vibração molecular. 


\section{Capítulo 3}

\section{Álgebras de Lie}

\subsection{Introdução às Álgebras de Lie}

Uma álgebra de Lie é por definição um espaço vetorial $L$ sobre um corpo $K$ real ou complexo no qual é definido uma operação binária [. , .] , chamada de comutador que satisfaz as seguintes propriedades:

1. $[\alpha X+\beta Y, Z]=\alpha[X, Y]+\beta[Y, Z]$ para $\alpha, \beta \in K$ (Bilinearidade)

2. $[X, Y]=-[Y, X]$ (Anti-simetria)

3. $[X,[Y, Z]]+[Y,[Z, X]]+[Z,[X, Y]]=0$ para todos $X, Y, Z \in L$ (Identidade de Jacobi).

O produto de dois geradores quaisquer de uma álgebra deve ser ainda um outro gerador dessa álgebra e, portanto deve ser uma combinação linear de todos geradores da álgebra

$$
\left[X_{\rho}, X_{\sigma}\right]=\sum_{\tau} c_{\rho \sigma}^{\tau} X_{\tau}
$$

as constantes $c_{\rho \sigma}^{\tau}$ são conhecidas como constantes de estrutura da álgebra com $c_{\rho \sigma}^{\tau}=-c_{\sigma p}^{\tau}$.

Uma álgebra de Lie é comutativa ou abeliana se para todos geradores $X, Y \in L$ nós temos $[X, Y]=0$.

Os protótipos das álgebras de Lie são os conjuntos $g l(n, C)$ de todas as matrizes $(n \times n)$ complexas as quais sob adição e multiplicação por escalar formam um espaço vetorial complexo de dimensão $n^{2}$ no qual o comutador é derivado da multiplicação de matrizes pela fórmula 
$[X, Y]=X Y-Y X$.

Uma base muito simples para essa álgebra foi dada por Weyl . Cada geradores da base será uma matriz $e_{i j}, i, j=1,2, \ldots, n$ contendo apenas um elemento diferente de zero e igual a um na posição $i j$ :

$$
\left(e_{i j}\right)_{m n}=\delta_{i m} \delta_{n j} .
$$

Assim, por exemplo, uma base para álgebra $g l(2, C)$ é formada pelas quatro matrizes $2 \times 2$ $e_{I I}=\left(\begin{array}{ll}1 & 0 \\ 0 & 0\end{array}\right) \quad e_{22}=\left(\begin{array}{ll}0 & 0 \\ 0 & 1\end{array}\right) \quad e_{12}=\left(\begin{array}{ll}0 & 1 \\ 0 & 0\end{array}\right) \quad e_{2 l}=\left(\begin{array}{ll}0 & 0 \\ 1 & 0\end{array}\right)$,

de forma que qualquer matriz complexa de dimensão 2 possa ser escrita como uma combinação linear delas.

Em geral, definimos uma álgebra de Lie a partir das relações de comutação entre seus geradores. No caso da álgebra $g l(n)$, cada gerador é escrito da forma $\left(e_{i j}\right)_{m n}=\delta_{i m \imath} \delta_{n j}$. Seja $\left[e_{a b}, e_{c d}\right]_{m n}$ um gerador de $g l(n)$, temos que

$$
\begin{aligned}
{\left[e_{a b}, e_{c d}\right]_{m n} } & =\left(e_{a b}, e_{c d}\right)_{m n}-\left(e_{c d} e_{a b}\right)_{m n} \\
& =\left(e_{a b}\right)_{m j}\left(e_{c d}\right)_{j n}-\left(e_{c d}\right)_{m j}\left(e_{a b}\right)_{i n} \\
& =\sum_{j} \delta_{a m} \delta_{b j} \delta_{c j} \delta_{d n}-\delta_{c m} \delta_{d j} \delta_{a j} \delta_{b n} \\
& =\delta_{a m} \delta_{b c} \delta_{d n}-\delta_{c m} \delta_{d a} \delta_{b n}=\delta_{b c} e_{a d}-\delta_{d a} e_{c b}
\end{aligned}
$$

Portanto, a relação de comutação para a álgebra $g l(n)$ é

$$
\left[e_{a b}, e_{c d}\right]=\delta_{b c} e_{a d}-\delta_{d a} e_{c b} .
$$

No caso da álgebra $g l(2)$ as relações de comutação são:

$$
\begin{array}{ll}
{\left[e_{11}, e_{22}\right]=0} & {\left[e_{11}, e_{22}\right]=e_{11}-e_{22}} \\
{\left[e_{11}, e_{12}\right]=e_{12}} & {\left[e_{11}, e_{21}\right]=-e_{21}} \\
{\left[e_{22}, e_{12}\right]=-e_{12}} & {\left[e_{22}, e_{21}\right]=e_{21} .}
\end{array}
$$

Observa-se que o conjunto das matrizes da forma $e_{i i}$ são comutantes e que o comutador entre um gerador deste tipo e um outro qualquer é sempre proporcional a esse outro. Neste caso, os geradores $e_{i i}$ formam uma subálgebra conhecida como subálgebra de Cartan, e as relações de comutação estão numa forma canônica conhecida como forma canônica de Cartan Weyl. 
Um dos problemas centrais na teoria das álgebras de Lie é a determinação e classificação de todas álgebras de Lie não isomorfas. As álgebras matriciais $A_{n}, B_{n}, C_{n}, D_{n}$ proporcionam grandes classes de álgebras de Lie. Estas álgebras matriciais esgotam todas as possíveis álgebras de Lie matriciais $\left(\mathrm{Ado}^{20}\right.$, 1947). Além destas, Killing e Cartan mostraram que há apenas mais cinco tipos de álgebras de Lie, denominadas de álgebras de Lie excepcionais, denotadas por $E_{6}, E_{7}, E_{8}, F_{4}$ e $G_{2}$.

\section{2 Álgebras de Lie Clássicas}

Apresentaremos nesta seção álgebras de Lie matriciais clássicas. Iniciaremos apresentando a simetria $A_{n-1}$ que se refere a álgebra $s l(n)$ de $\operatorname{rank} r=n-1$ e dimensão $r(r+2)$ correspondente ao conjunto de matrizes de traço nulo:

$$
\operatorname{Tr} X=0,
$$

onde $X$ é um gerador da álgebra.

Podemos dar uma base para as matrizes de traço nulo, a partir da base de Weyl (3.3). Basta tomarmos

$$
A_{i i}=e_{i i}-e_{i+1, i+1}, A_{i j}=e_{i j},
$$

onde os $A_{i j}$ são geradores da base de $s l(n)$ e os $e_{i j}$ são geradores da base de Weyl. A partir da base de Weyl podemos escrever as relações de comutaçăo desta álgebra, ou seja:

$$
\begin{aligned}
{\left[A_{i i}, A_{k l}\right] } & =\left[e_{i i}-e_{i+1, i+1}, e_{k l}\right] \\
& =\left[e_{i i}, e_{k l}\right]-\left[e_{i+1, i+1}, e_{k l}\right] \\
& =\delta_{k i} e_{i l}-\delta_{i l} e_{k i}-\delta_{k, i+1} e_{i+1, l}-\delta_{i+1, l} e_{k, i+1} \quad k \neq l \\
& =\delta_{k i} e_{k l}-\delta_{i l} e_{k l}-\delta_{k, i+1} e_{k, l}-\delta_{i+1, l} e_{k, l} \\
& =\left(\delta_{k i}-\delta_{i l}-\delta_{k, i+1}-\delta_{i+1, l}\right) A_{k l}
\end{aligned}
$$

$$
\begin{aligned}
{\left[A_{i j}, A_{j i}\right] } & =\left[e_{i j}, e_{j i}\right] \\
& =\delta_{j j} e_{i i}-\delta_{i i} e_{j j} \\
& =e_{i i}-e_{j j}
\end{aligned}
$$

$\left[A_{i i}, A_{j j}\right]=\left[e_{i i}-e_{i+1, i+1}, e_{j j}-e_{j+1, j+1}\right]$ 


$$
\begin{aligned}
= & {\left[e_{i i}, e_{j j}\right]-\left[e_{i+1, i+1}, e_{j j}\right]-\left[e_{i i}, e_{j+l, j+1}\right]+\left[e_{i+l, i+1}, e_{j+l, i+1}\right] } \\
= & \delta_{j i} e_{i j}-\delta_{i j} e_{j i}-\delta_{j, i+1} e_{i+l, j}+\delta_{l+l, j} e_{j, i+1}-\delta_{j+l, i} e_{i, j+1}+\delta_{i, j+1} e_{j+l, i}+\delta_{j+l, i+l} e_{i+l, j+l} \\
& +\delta_{i+l, j+l} e_{j+1, i+1}=0
\end{aligned}
$$

$$
\begin{aligned}
{\left[A_{i j}, A_{k l}\right] } & =\left[e_{i j}, e_{k l}\right] \\
& =\delta_{k j} e_{i l}-\delta_{i l} e_{k j} \\
& =\delta_{k j} A_{i l}-\delta_{i l} A_{k j} .
\end{aligned}
$$

Portanto a álgebra $s l(n)$ apresenta as seguintes relaçōes de comutaçāo

$$
\begin{aligned}
& {\left[A_{i i}, A_{k l}\right]=\left(\delta_{k i}-\delta_{i l}-\delta_{k, i+1}-\delta_{i+1, l}\right) A_{k l}} \\
& {\left[A_{i j}, A_{j i}\right]=e_{i i}-e_{j j}} \\
& {\left[A_{i i}, A_{j j}\right]=0} \\
& {\left[A_{i j}, A_{k l}\right]=\delta_{k j} A_{i l}-\delta_{i l} A_{k j} .}
\end{aligned}
$$

Por exemplo, a álgebra $s l(2)$ formada pelas matrizes

$$
A_{I I}=\left(\begin{array}{cc}
1 & 0 \\
0 & -1
\end{array}\right), \quad A_{l 2}=\left(\begin{array}{ll}
0 & 1 \\
0 & 0
\end{array}\right), \quad A_{2 L}=\left(\begin{array}{ll}
0 & 0 \\
1 & 0
\end{array}\right),
$$

apresenta as relações de comutação

$$
\begin{aligned}
& {\left[A_{11}, A_{12}\right]=2 A_{12}} \\
& {\left[A_{11}, A_{21}\right]=-2 A_{21}} \\
& {\left[A_{12}, A_{21}\right]=A_{11} .}
\end{aligned}
$$

Note que as relações já estão na forma de Cartan-Weyl, com $A_{\|}$pertencendo à subálgebra de Cartan.

Agora mostraremos as simetrias $\boldsymbol{B}_{n}$ e $\boldsymbol{D}_{n}$ correspondentes as álgebras ortogonais so(n), formada por matrizes anti-simétricas de dimensão ímpar e par, respectivamente:

$$
X^{T}=-X,
$$

onde $X^{T}$ significa transposição. Assim o conjunto das matrizes complexas anti-simétricas de dimensão ímpar formam a álgebra ortogonal $o(2 n+1, C)$ ou $B_{n}$. Aquelas de dimensão par formam a álgebra ortogonal $o(2 n, C)$ ou $D_{n}$. Caso nos restrinjamos às matrizes anti-simétricas de traço nulo, teremos as álgebras de Lie ortogonais especiais, $s o(2 n+1)$ para $B_{n}$ e $s o(2 n)$ para $D_{n}$. Esta álgebra tem rank $r=n$ e dimensão $n(2 n+1)$ e $n(2 n-1)$ respectivamente. Como vimos 
nos casos anteriores podemos construir uma base para as álgebras ortogonais especiais a partir da base de Weyl (3.3):

$$
X_{i j}=A_{i j}-A_{j i}, i \neq j=1,2, \ldots, n .
$$

Por exemplo para a álgebra $s o(3)$, temos os geradores

$$
X_{12}=\left(\begin{array}{ccc}
0 & 1 & 0 \\
-1 & 0 & 0 \\
0 & 0 & 0
\end{array}\right), X_{13}=\left(\begin{array}{ccc}
0 & 0 & 1 \\
0 & 0 & 0 \\
-1 & 0 & 0
\end{array}\right), X_{23}=\left(\begin{array}{ccc}
0 & 0 & 0 \\
0 & 0 & 1 \\
0 & -1 & 0
\end{array}\right) \text {. }
$$

As relaçōes de comutação para estas matrizes são:

$\left[X_{12}, X_{13}\right]=-X_{23}$,

$\left[X_{12}, X_{23}\right]=X_{13}$,

$\left[X_{23}, X_{13}\right]=X_{12}$.

Podemos re-escrever estas relaçōes numa forma mais apropriada tomando

$L_{1}=X_{23}, L_{2}=X_{13}, L_{3}=X_{12}$.

Assim,

$\left[L_{j}, L_{k}\right]=\varepsilon_{j k m} L_{m}$.

Apesar destas relações de comutação não estarem na forma canônica, isto pode ser feito facilmente, basta tomarmos novos elementos

$$
J_{0}=i L_{3}, \quad J_{ \pm}=\frac{i}{\sqrt{2}}\left(L_{1} \pm i L_{2}\right) \text {. }
$$

Dessa forma, temos as relaçōes de comutação na forma canônica

$$
\left[J_{0}, J_{ \pm}\right]= \pm J_{ \pm},\left[J_{+}, J_{-}\right]=J_{0} \text {. }
$$

Em geral, não é uma tarefa fácil re-escrever os elementos das álgebras ortogonais na forma canônica.

Quanto a simetria $C_{n}$ ela é formada pelas álgebras de Lie $\operatorname{sp}(2 n, C)$, isto é, pelo conjunto de matrizes complexas $X(2 n \times 2 n)$ satisfazendo

$$
X^{T} \omega+\omega X=0
$$

onde as matrizes $X$ e $\omega$ têm as formas:

$X=\left(\begin{array}{cc}A & B \\ C & -A^{T}\end{array}\right), \quad \omega=\left(\begin{array}{cc}0 & 1_{n} \\ -1_{n} & 0\end{array}\right)$,

e $A, B$ e $C$ são matrizes complexas simétricas (nxn). A dimensão desta álgebra de Lie é $n(2 n+1)$. 
A álgebra de Lie $s p(2 n)$ unitária é obtida impondo que as matrizes envolvidas sejam anti-hermitianas, ou seja,

$$
X^{T}+X=0 \quad \text { ou } \quad A^{T}+A=0 \quad, \quad B^{T}+C=0=C^{T}+B,
$$

e a álgebra de Lie $s p(2 n, R)$ é obtida impondo que as matrizes envolvidas sejam todas reais ao invés de complexas.

As relações de comutação para a álgebra de Lie simplética pode ser definida pela manipulação direta de matrizes. Lembrando a relação útil da base de Weyl

$$
e_{i j} e_{k l}=\delta_{j k} e_{i l},
$$

os geradores de $s p(2 n, C)$ podem ser escritos em termos das matrizes (2nx2n) abaixo expressadas na forma de blocos $(2 \times 2)$ :

$$
\begin{array}{rlr}
A_{i j} & =\left(\begin{array}{cc}
e_{i j} & 0 \\
0 & -e_{j i}
\end{array}\right) & (1 \leq i, j \leq n), \\
B_{i j} & =\left(\begin{array}{cc}
0 & e_{i j}+e_{j i} \\
0 & 0
\end{array}\right) & (1 \leq i \leq j \leq n), \\
C_{i j} & =\left(\begin{array}{cc}
0 & 0 \\
e_{i l}+e_{j i} & 0
\end{array}\right) & (1 \leq i \leq j \leq n) .
\end{array}
$$

Assim podemos escrever as relações de comutação para esta álgebra definindo

$$
\begin{aligned}
& A_{i j}=e_{i j}-e_{j+n, i+n}, \\
& B_{i j}=e_{i, j+n}+e_{j, i+n}, \\
& C_{i j}=e_{i+n, j}+e_{j+n, i}, \\
& \text { então, }
\end{aligned}
$$

$$
\begin{aligned}
{\left[A_{i j}, A_{k l}\right]=} & {\left[e_{i j}-e_{j+n, i+n}, e_{k l}-e_{l+n, k+n}\right] } \\
= & {\left[e_{i j}, e_{k l}\right]-\left[e_{i j}, e_{l+n, k+n}\right]-\left[e_{j+n, i+n}, e_{k l}\right]+\left[e_{j+n, i+n}, e_{l+n, k+n}\right] } \\
= & \delta_{k j} e_{i l}-\delta_{i l} e_{k j}-\delta_{l+n, j} e_{i, k+n}+\delta_{i, k+n} e_{l+n, j}-\delta_{k, i+n} e_{j+n, l}+\delta_{j+n, l} e_{k, i+n}+\delta_{l+n, i+n} e_{j+n, k+n} \\
& -\delta_{j+n, k+n} e_{l+n, l+n} \\
= & \delta_{k j} e_{i l}-\delta_{i l} e_{k j}+\delta_{l+n, i+n} e_{j+n, k+n}-\delta_{j+n, k+n} e_{l+n, i+n} \\
= & \delta_{k j} A_{i l}-\delta_{i l} A_{k j}
\end{aligned}
$$

$$
\begin{aligned}
{\left[A_{i j}, B_{k l}\right] } & =\left[e_{i j}-e_{j+n, i+n}, e_{k, l+n}+e_{l, k+n}\right] \\
& =\left[e_{i j}, e_{k, l+n}\right]+\left[e_{i j}, e_{l, k+n}\right]-\left[e_{j+n, i+n}, e_{k, l+n}\right]-\left[e_{j+n, i+n}, e_{l, k+n}\right]
\end{aligned}
$$




$$
\begin{aligned}
= & \delta_{k j} e_{i, l+n}-\delta_{l, l+n} e_{k j}+\delta_{l j} e_{i, k+n}-\delta_{i, k+n} e_{l j}-\delta_{k, i+n} e_{j+n, l+n}+\delta_{j+n, l+n} e_{k, i+n}-\delta_{l, i+n} e_{j+n, k+n} \\
& +\delta_{j+n, k+n} e_{l, i+n} \\
= & \delta_{j+n, l+n} e_{k, i+n}+\delta_{j+n, k+n} e_{l, i+n}+\delta_{k j} e_{i, l+n}+\delta_{l j} e_{i, k+n} \\
= & \delta_{k j} B_{i l}-\delta_{j l} B_{i k}
\end{aligned}
$$

$$
\begin{aligned}
{\left[A_{i j}, C_{k l}\right]=} & {\left[e_{i j}-e_{j+n, i+n}, e_{k+n, l}+e_{l+n, k}\right] } \\
= & {\left[e_{i j}, e_{k+n, l}\right]+\left[e_{i j}, e_{l+n, k}\right]-\left[e_{j+n, i+n}, e_{k+n, l}\right]-\left[e_{j+n, i+n}, e_{l+n, k}\right] } \\
= & \delta_{k+n, j} e_{i l}-\delta_{i l} e_{k+n j}+\delta_{l+n, j} e_{i, k}-\delta_{i, k} e_{l+n, j}-\delta_{k+n, i+n} e_{j+n, l}+\delta_{j+n, l} e_{k+n, i+n}-\delta_{l+n, i+n} e_{j+n, k}+ \\
& +\delta_{j+n, k} e_{l+n, i+n} \\
= & -\delta_{i l} e_{k+n j}-\delta_{i, k} e_{l+n, j}-\delta_{k+n, i+n} e_{j+n, l}-\delta_{l+n, i+n} e_{j+n, k} \\
= & -\delta_{i k} C_{j l}-\delta_{i l} C_{j k}
\end{aligned}
$$

$$
\begin{aligned}
{\left[B_{i j} B_{k l}\right]=} & {\left[e_{i, j+n}+e_{j, i+n}, e_{k, l+n}+e_{l, k+n}\right] } \\
= & {\left[e_{i, j+n}, e_{k, l+n}\right]+\left[e_{i, j+n}, e_{l, k+n}\right]+\left[e_{j, i+n}, e_{k, l+n}\right]+\left[e_{j, i+n}, e_{l, k+n}\right] } \\
= & \delta_{k, j+n} e_{i, l+n}-\delta_{l, l+n} e_{k, j+n}+\delta_{l, j+n} e_{i, k+n}-\delta_{i, k+n} e_{l, j+n}+\delta_{k, i+n} e_{j, l+n}-\delta_{j, l+n} e_{k, i+n}+\delta_{l, i+n} e_{j, k+n}- \\
& -\delta_{j, k+n} e_{l, i+n} \\
= & 0
\end{aligned}
$$

$$
\begin{aligned}
{\left[C_{i j}, C_{k l}\right]=} & {\left[e_{i+n, j}+e_{j+n, i}, e_{k+n, l}+e_{l+n, k}\right] } \\
= & {\left[e_{i+n, j}, e_{k+n, l}\right]+\left[e_{i+n, j}, e_{l+n, k}\right]+\left[e_{j+n, i}, e_{k+n, l}\right]+\left[e_{j+n, i}, e_{l+n, k}\right] } \\
= & \delta_{k+n, j} e_{i+n, l}-\delta_{i+n, l} e_{k+n, j}+\delta_{l+n, j} e_{i+n, k}-\delta_{i+n, k} e_{l+n, j}+\delta_{k+n, i} e_{j+n, l}-\delta_{j+n, l} e_{k+n, i}+\delta_{l+n, i} e_{j+n, k}- \\
& -\delta_{j+n, k} e_{l+n, i} \\
= & 0
\end{aligned}
$$

$$
\begin{aligned}
{\left[B_{i j}, C_{k l}\right]=} & {\left[e_{i, j+n}+e_{j, i+n}, e_{k+n, l}+e_{l+n, k}\right] } \\
= & {\left[e_{i, j+n}, e_{k+n, l}\right]+\left[e_{i, j+n}, e_{l+n, k}\right]+\left[e_{j, i+n}, e_{k+n, l}\right]+\left[e_{j, i+n}, e_{l+n, k}\right] } \\
= & \delta_{k+n, j+n} e_{i, l}-\delta_{i, l} e_{k+n, j+n}+\delta_{l+n, j+n} e_{i, k}-\delta_{i, k} e_{l+n, j+n}+\delta_{k+n, i+n} e_{j, l}-\delta_{j, l} e_{k+n, i+n}+\delta_{l+n, i+n} e_{j, k}- \\
& -\delta_{j k} e_{l+n, i+n} \\
= & \delta_{i k} A_{j l}+\delta_{j k} A_{i l}+\delta_{i l} A_{j k}+\delta_{j l} A_{i k}
\end{aligned}
$$

Portanto temos as seguintes relações de comutação para a álgebra simplética: 
1- $\left[A_{i j}, A_{k l}\right]=\delta_{k j} A_{i l}-\delta_{i l} A_{k j}$

$2-\left[A_{i j}, B_{k l}\right]=\delta_{k j} B_{i l}-\delta_{j l} B_{i k}$

3- $\left[A_{i j}, C_{k l}\right]=-\delta_{i k} C_{j l}-\delta_{i l} C_{j k}$

$4-\left[B_{i j}, B_{k l}\right]=0$

5- $\left[C_{i j}, C_{k l}\right]=0$

6- $\left[B_{i j}, C_{k l}\right]=\delta_{i k} A_{j l}+\delta_{j k} A_{i l}+\delta_{i l} A_{j k}+\delta_{j l} A_{i k}$.

Nas próximas seções apresentaremos alguns resultados e definições muito importantes para o entendimento das álgebras de Lie.

\section{3 Álgebras de Lie e Subálgebras}

Um conjunto $H$ de uma álgebra de Lie $A$ é chamado de subálgebra de $A$ se $H$ é um subespaço de $A$ e satisfaz, $[X, Y] \in H$ para qualquer $X, Y \in H$, e uma subálgebra $H$ de $A$ é dita ser Abeliana se $[X, Y]=O$ para qualquer $X, Y \in H$.

\subsection{Ideais e Ideais Próprios}

Um subconjunto $H$ de $A$ é um ideal ou uma subálgebra invariante de $A$ se $H$ é um subespaço linear de $A$ e o comutador $[X, Y] \in H$ para qualquer $X \in H, Y \in A$, isto é,

$$
\left[X_{\rho}, X_{\sigma}\right]=\sum_{\tau} c_{\rho \sigma}^{\tau} X_{\tau} \quad \rho, \tau \in H, \sigma \in A
$$

Um ideal próprio é todo ideal com exceção da álgebra inteira e do elemento nulo. Restringindo as atenções aos ideais próprios eliminamos os ideais impróprios formado pela álgebra inteira e pelo subconjunto contendo apenas o elemento nulo.

$O$ conjunto de todos os geradores $X_{\sigma}$ de uma álgebra $A$ que satisfaz a condição

$$
\left[X_{\rho}, X_{\sigma}\right]=0 \quad \operatorname{com} \rho \in H, \sigma \in A,
$$

é dito formar o ideal maximal ou centro da álgebra. Os geradores do centro de uma álgebra formam uma subálgebra Abeliana, e que comuta com todos os geradores do grupo $G$. 


\subsection{Representações Adjuntas das Álgebras de Lie}

Fixando qualquer gerador $X$ de uma álgebra de Lie $A$ definimos uma transformação linear chamada adjunta (ad):

$\operatorname{ad}(X): Z \rightarrow[X, Z]$, para qualquer $Z \in A$.

Considere qualquer $K \in A$, então

$$
\begin{aligned}
{[\operatorname{ad}(Y), \operatorname{ad}(Z)] K } & =\operatorname{ad}(Y) \operatorname{ad}(Z) K-\operatorname{ad}(Z) \operatorname{ad}(Y) K \\
& =\operatorname{ad}(Y)[Z, K]-\operatorname{ad}(Z)[Y, K] \\
& =[Y,[Z, K]]-[Z,[Y, K]] \\
& =[[Y, Z], K] \\
& =\operatorname{ad}([Y, Z]) K
\end{aligned}
$$

onde usamos a identidade de Jacobi. A transformação "ad" nos dá uma representação da álgebra de Lie conhecida como representação adjunta.

Como exemplo, temos para álgebra so(3), cujos geradores são $L_{1}, L_{2}$ e $L_{3}$ satisfazendo a relação de comutação

$$
\left[L_{i}, L_{j}\right]=\varepsilon_{i j l} L_{l} .
$$

Daí,

$\left(\operatorname{ad} L_{l}\right) L_{l}=\left[L_{1}, L_{l}\right]=0$

$\left(\operatorname{ad} L_{1}\right) L_{2}=\left[L_{1}, L_{2}\right]=L_{3} \quad \Rightarrow a d L_{1} \rightarrow\left(\begin{array}{ccc}0 & 0 & 0 \\ 0 & 0 & -1 \\ 0 & 1 & 0\end{array}\right)=R_{1}$,

$\left(\operatorname{ad} L_{1}\right) L_{3}=\left[L_{1}, L_{3}\right]=-L_{2}$

$\left(\operatorname{ad} L_{2}\right) L_{1}=\left[L_{2}, L_{1}\right]=-L_{3}$

$\left(a d L_{2}\right) L_{2}=\left[L_{2}, L_{2}\right]=0 \quad \Rightarrow a d L_{1} \rightarrow\left(\begin{array}{ccc}0 & 0 & 1 \\ 0 & 0 & 0 \\ -1 & 0 & 0\end{array}\right)=R_{2}$,

$\left(\operatorname{ad} L_{2}\right) L_{3}=\left[L_{2}, L_{3}\right]=L_{1}$

$\left(\operatorname{ad} L_{3}\right) L_{J}=\left[L_{3}, L_{1}\right]=L_{2}$ 
$\left(a d L_{3}\right) L_{2}=\left[L_{3}, L_{2}\right]=-L_{1} \quad \Rightarrow a d L_{1} \rightarrow\left(\begin{array}{ccc}0 & -1 & 0 \\ 1 & 0 & 0 \\ 0 & 0 & 0\end{array}\right)=R_{3}$.

$\left(\right.$ ad $\left.L_{3}\right) L_{3}=\left[L_{3}, L_{3}\right]=0$

Portanto, podemos escrever as relações de comutação como:

$$
\left[R_{i}, R_{j}\right]=\varepsilon_{i j l} R_{i} .
$$

\subsection{Soma Direta e Soma Semi-Direta}

O conceito de soma de álgebras é importante pois nos dá noções de decomposição de uma álgebra de Lie, o que leva a classificação de possíveis simetrias.

Uma álgebra de Lie $A$ é dividida em soma direta de subálgebras de Lie se para todo par de subálgebras $A_{i}, A_{j}$ temos $A_{i} \cap A_{j}=0, i, j=1,2, \ldots, n$ e é denotada por

$$
A=A_{1} \oplus A_{2} \oplus \ldots \oplus A_{n} .
$$

Se uma álgebra de Lie $A$ tem duas subálgebras $A_{1}, A_{2}$ tal que

$$
\left[A_{1}, A_{1}\right] \subset A_{1},\left[A_{2}, A_{2}\right] \subset A_{2},\left[A_{1}, A_{2}\right] \subset A_{1},
$$

então a álgebra de Lie $A$ é dita ser a soma semi-direta de $A_{1}$ e $A_{2}$, onde $A_{1}$ é um ideal da soma semi-direta. Normalmente nós escrevemos uma soma semi-direta primeiro dando o ideal e então a subálgebra residual, dessa forma

$$
A=A_{1} \oplus{ }_{s} A_{2}
$$

\section{7 Álgebras de Lie Solúveis e Nilpotentes}

Nesta seção apresentaremos os conceitos de álgebras de Lie solúveis e álgebras de Lie Nilpotentes. Para facilitar tais definições citaremos o seguinte teorema (Dynkin) e faremos alguns exemplos. Denotemos então por $T^{(m)}$ o espaço vetorial das matrizes $(m \times m)$ triangulares superiores com elementos diagonais iguais e por $S^{m_{1}, m_{2} \ldots, m_{k}} \mathrm{o}$ conjunto de todas transformaçōes lineares $A$ agindo no espaço

$$
V=V_{1}+V_{2}+\cdots+V_{K},
$$


de tal maneira que

i) $A \in S^{m_{1}, m_{2}, \ldots, m_{k}}$ deixe os subespaços $V_{i}$ invariantes;

ii) Em cada subespaços $V_{i}$ com base $\xi_{1}^{i}, \xi_{2}^{i}, \ldots, \xi_{k}^{i}, A \in V_{i}$

$$
\left(\begin{array}{llll}
\gamma_{i} & & & a_{i j} \\
& \gamma_{i} & & \\
& & \ddots & \\
0 & & & \gamma_{i}
\end{array}\right)
$$

Com estas definiçōes estabelecemos o seguinte teorema:

Teorema 1 Uma álgebra de Lie solúvel arbitrária é isomorfa a uma subálgebra de alguma álgebra de Lie $T^{(m)}$. Uma álgebra de Lie nilpotente é isomorfa a uma subálgebra de alguma álgebra de Lie $S^{m_{1}, m_{2} \ldots m_{2}}$.

Consideremos então, a título de exemplo, as matrizes

$$
B=\left(\begin{array}{cccc}
a_{1} & b_{1} & c_{1} & d_{1} \\
0 & e_{1} & f_{1} & g_{1} \\
0 & 0 & h_{1} & i_{1} \\
0 & 0 & 0 & j_{1}
\end{array}\right), \quad B^{\prime}=\left(\begin{array}{cccc}
a_{2} & b_{2} & c_{2} & d_{2} \\
0 & e_{2} & f_{2} & g_{2} \\
0 & 0 & h_{2} & i_{2} \\
0 & 0 & 0 & j_{2}
\end{array}\right) \text {, }
$$

multiplicando $B$ por $B^{\prime}$ e $B^{\prime}$ por $B$ temos respectivamente

$$
\begin{aligned}
& B B^{\prime}=\left(\begin{array}{cccc}
a_{1} a_{2} & a_{1} b_{2}+b_{1} e_{2} & a_{1} c_{2}+b_{1} f_{2}+c_{1} h_{2} & a_{1} d_{2}+b_{1} g_{2}+c_{1} i_{2}+d_{1} j_{2} \\
0 & e_{1} e_{2} & e_{1} f_{2}+f_{1} h_{2} & e_{1} g_{2}+f_{1} i_{2}+g_{1} j_{2} \\
0 & 0 & h_{1} h_{2} & h_{1} i_{2}+i_{1} j_{2} \\
0 & 0 & 0 & j_{1} j_{2}
\end{array}\right), \\
& B^{\prime} B=\left(\begin{array}{cccc}
a_{1} a_{2} & a_{2} b_{1}+b_{2} e_{1} & a_{2} c_{1}+b_{2} f_{1}+c_{2} h_{1} & a_{2} d_{1}+b_{2} g_{1}+c_{2} i_{1}+d_{2} j_{1} \\
0 & e_{1} e_{2} & e_{2} f_{1}+f_{2} h_{1} & e_{2} g_{1}+f_{2} i_{1}+g_{2} j_{1} \\
0 & 0 & h_{1} h_{2} & h_{2} i_{1}+i_{2} j_{1} \\
0 & 0 & 0 & j_{1} j_{2}
\end{array}\right),
\end{aligned}
$$

logo o comutador de duas matrizes do tipo $B$ é uma matriz do tipo $C$

$$
\left[B, B^{\prime}\right]=C=\left(\begin{array}{cccc}
0 & a & b & c \\
0 & 0 & d & e \\
0 & 0 & 0 & f \\
0 & 0 & 0 & 0
\end{array}\right),
$$

sendo $C^{\prime}$ e $C^{\prime \prime}$ matrizes da forma de $C$ 


$$
C^{\prime}=\left(\begin{array}{cccc}
0 & a_{3} & b_{3} & c_{3} \\
0 & 0 & d_{3} & e_{3} \\
0 & 0 & 0 & f_{3} \\
0 & 0 & 0 & 0
\end{array}\right), \quad C^{\prime \prime}=\left(\begin{array}{cccc}
0 & a_{4} & b_{4} & c_{4} \\
0 & 0 & d_{4} & e_{4} \\
0 & 0 & 0 & f_{4} \\
0 & 0 & 0 & 0
\end{array}\right)
$$

multiplicando $C^{\prime}$ por $C^{\prime \prime}$ e $C^{\prime \prime}$ por $C^{\prime}$ temos respectivamente

$$
C^{\prime} C^{\prime \prime}=\left(\begin{array}{cccc}
0 & 0 & a_{3} d_{4} & a_{3} e_{4}+b_{3} f_{4} \\
0 & 0 & 0 & d_{3} f_{4} \\
0 & 0 & 0 & 0 \\
0 & 0 & 0 & 0
\end{array}\right), \quad C^{\prime \prime} C^{\prime}=\left(\begin{array}{cccc}
0 & 0 & a_{4} d_{3} & a_{4} e_{3}+b_{4} f_{3} \\
0 & 0 & 0 & d_{4} f_{3} \\
0 & 0 & 0 & 0 \\
0 & 0 & 0 & 0
\end{array}\right),
$$

então o comutador de duas matrizes do tipo $C$ é uma matriz do tipo $D$

$$
\left[C^{\prime}, C^{\prime \prime}\right]=D=\left(\begin{array}{cccc}
0 & 0 & a_{3} d_{4}-a_{4} d_{3} & a_{3} e_{4}+b_{3} f_{4}-a_{4} e_{3}+b_{4} f_{3} \\
0 & 0 & 0 & d_{3} f_{4}-d_{4} f_{3} \\
0 & 0 & 0 & 0 \\
0 & 0 & 0 & 0
\end{array}\right),
$$

sendo $D^{\prime}$ e $D^{\prime \prime}$ matrizes da forma de $D$

$$
D^{\prime}=\left(\begin{array}{cccc}
0 & 0 & a & b \\
0 & 0 & 0 & c \\
0 & 0 & 0 & 0 \\
0 & 0 & 0 & 0
\end{array}\right), \quad D^{\prime \prime}=\left(\begin{array}{cccc}
0 & 0 & d & e \\
0 & 0 & 0 & f \\
0 & 0 & 0 & 0 \\
0 & 0 & 0 & 0
\end{array}\right)
$$

calculando o comutador de $D^{\prime}$ e $D^{\prime \prime}$ obtemos

$$
\left[D^{\prime}, D^{\prime \prime}\right]=\left(\begin{array}{cccc}
0 & 0 & 0 & 0 \\
0 & 0 & 0 & 0 \\
0 & 0 & 0 & 0 \\
0 & 0 & 0 & 0
\end{array}\right)
$$

Tendo em vista a propriedade acima, dizemos que as matrizes da forma de $B$ são solúveis. Formalmente, se $N$ é um ideal de uma álgebra de Lie $A$, então $[N, N]$ é também um ideal de $A$, de fato,

$$
[A,[N, N]] \subset[N,[N, A]]+[N,[A, N]] \subset[N, N],
$$

em particular $A$ é um ideal de $A$ e portanto $[A, A]$ é ainda um ideal de $A$, logo pode acontecer que a seqüência de ideais

$$
A^{(0)}=A, A^{(l)}=\left[A^{(0)}, A^{(0)}\right], \ldots, A^{(k)}=\left[A^{(k-1)}, A^{(k-1)}\right], \quad k=0,1,2, \ldots
$$

termine em $O$, isto é, $A^{(k)}=0$, para algum $k$. Definimos então, que uma álgebra de Lie é chamada solúvel se para algum inteiro positivo $k, A^{(k)}=0$. 
Como exemplo, consideremos a álgebra de Lie $e(2)$ dos geradores do grupo Euclidiano $E(3)$ em duas dimensões: $\left\{P_{x}, P_{y}, L_{z}\right\}$. Calculemos, sendo

$$
\begin{aligned}
& A=\alpha_{1} P_{x}+\alpha_{2} P_{y}+\alpha_{3} L_{z} \\
& B=\beta_{1} P_{x}+\beta_{2} P_{y}+\beta_{3} L_{z}
\end{aligned}
$$

o comutador

$$
[A, B]=\alpha_{3} \beta_{1}\left[L_{z}, P_{x}\right]+\alpha_{3} \beta_{2}\left[L_{z}, P_{y}\right]-\alpha_{2} \beta_{3}\left[L_{z}, P_{y}\right]-\alpha \beta\left[L_{z}, P_{x}\right]=f\left(P_{x}, P_{y}\right) .
$$

Como a álgebra de $(P)$ é comutativa, $A^{(2)}=0$. Portanto $e(2)$ é solúvel.

Observaremos agora o comportamento das matrizes triangulares superiores de diagonal idêntica na seqüência de operações seguinte. Tomemos como exemplo matrizes $B$ e $B^{\prime}$

$$
B=\left(\begin{array}{cccc}
a_{1} & b_{1} & c_{1} & d_{1} \\
0 & a_{1} & e_{1} & f_{1} \\
0 & 0 & a_{1} & g_{1} \\
0 & 0 & 0 & a_{1}
\end{array}\right), \quad B^{\prime}=\left(\begin{array}{cccc}
a_{2} & b_{2} & c_{2} & d_{2} \\
0 & a_{2} & e_{2} & f_{2} \\
0 & 0 & a_{2} & g_{2} \\
0 & 0 & 0 & a_{2}
\end{array}\right)
$$

multiplicando $B$ por $B^{\prime}$ e $B^{\prime}$ por $B$ temos respectivamente

$$
\begin{aligned}
& B B^{\prime}=\left(\begin{array}{cccc}
a_{1} a_{2} & a_{1} b_{2}+b_{1} a_{2} & a_{1} c_{2}+b_{1} e_{2}+c_{1} a_{2} & a_{1} d_{2}+b_{1} f_{2}+c_{1} g_{2}+d_{1} a_{2} \\
0 & a_{1} a_{2} & a_{1} e_{2}+e_{1} a_{2} & a_{1} f_{2}+e_{1} g_{2}+f_{1} a_{2} \\
0 & 0 & a_{1} a_{2} & a_{1} g_{2}+g_{1} a_{2} \\
0 & 0 & 0 & a_{1} a_{2}
\end{array}\right), \\
& B^{\prime} B=\left(\begin{array}{cccc}
a_{1} a_{2} & a_{1} b_{2}+b_{1} a_{2} & c_{1} a_{2}+b_{2} e_{1}+a_{1} c_{2} & d_{1} a_{2}+b_{2} f_{1}+c_{2} g_{1}+a_{1} d_{2} \\
0 & a_{1} a_{2} & a_{1} e_{1}+e_{1} a_{2} & f_{1} a_{2}+e_{2} g_{1}+a_{1} f_{2} \\
0 & 0 & a_{1} a_{2} & a_{1} g_{2}+g_{1} a_{2} \\
0 & 0 & 0 & a_{1} a_{2}
\end{array}\right),
\end{aligned}
$$

logo o comutador de duas matrizes do tipo $B$ é uma matriz do tipo $C$

$$
\left[B, B^{\prime}\right]=C=\left(\begin{array}{cccc}
0 & 0 & b_{1} e_{2}-b_{2} e_{1} & b_{1} f_{2}+c_{1} g_{2}-b_{2} f_{1}-c_{2} g_{1} \\
0 & 0 & 0 & e_{1} g_{2}-e_{2} g_{1} \\
0 & 0 & 0 & 0 \\
0 & 0 & 0 & 0
\end{array}\right)
$$

calculando o comutador de uma matriz do tipo $B$ com uma do tipo $C$ temos

$$
B^{\prime \prime} C=\left(\begin{array}{cccc}
0 & 0 & a_{3}\left(b_{1} e_{2}-b_{2} e_{1}\right) & a_{3} b_{1} f_{2}+a_{3} c_{1} g_{2}-a_{3} b_{2} f_{1}-a_{3} c_{2} g_{1}+b_{3} e_{1} g_{2}-b_{3} e_{1} g_{1} \\
0 & 0 & 0 & a_{3}\left(e_{1} g_{2}-e_{2} g_{1}\right) \\
0 & 0 & 0 & 0 \\
0 & 0 & 0 & 0
\end{array}\right)
$$




$$
\begin{aligned}
& C B^{\prime \prime}=\left(\begin{array}{cccc}
0 & 0 & a_{3}\left(b_{1} e_{2}-b_{2} e_{1}\right) & g_{3} b_{1} e_{2}+g_{3} b_{2} e_{1}+a_{3} b_{1} f_{2}+a_{3} c_{1} g_{2}+a_{3} b_{2} f_{1}-a_{3} c_{2} g_{1} \\
0 & 0 & 0 & a_{3}\left(e_{1} g_{2}-e_{2} g_{1}\right) \\
0 & 0 & 0 & 0 \\
0 & 0 & 0 & 0
\end{array}\right) \\
& {\left[C, B^{\prime \prime}\right]=D=\left(\begin{array}{cccc}
0 & 0 & 0 & b_{3} e_{1} g_{2}-b_{3} e_{2} g_{1}-g_{3} b_{1} e_{2}+g_{3} b_{2} e_{1} \\
0 & 0 & 0 & 0 \\
0 & 0 & 0 & 0 \\
0 & 0 & 0 & 0
\end{array}\right)}
\end{aligned}
$$

finalmente, o comutador de uma matriz do tipo $B$ com uma do tipo $D$ se anula, de fato

$$
\begin{aligned}
B^{\prime \prime} D & =\left(\begin{array}{cccc}
0 & 0 & 0 & z a_{2} \\
0 & 0 & 0 & 0 \\
0 & 0 & 0 & 0 \\
0 & 0 & 0 & 0
\end{array}\right), \quad D B^{\prime \prime}=\left(\begin{array}{cccc}
0 & 0 & 0 & z a_{2} \\
0 & 0 & 0 & 0 \\
0 & 0 & 0 & 0 \\
0 & 0 & 0 & 0
\end{array}\right), \\
{\left[B^{\prime \prime}, D\right] } & =\left(\begin{array}{llll}
0 & 0 & 0 & 0 \\
0 & 0 & 0 & 0 \\
0 & 0 & 0 & 0 \\
0 & 0 & 0 & 0
\end{array}\right) .
\end{aligned}
$$

Dizemos que as matrizes do tipo $B$ são nilpotentes. Note que essas matrizes são solúveis também, de fato, toda álgebra de Lie nilpotente é necessariamente solúvel, mas uma álgebra de Lie solúvel não precisa ser nilpotente. Podemos expressar formalmente a propriedade acima definindo álgebras de Lie Nilpotentes, ou seja, uma álgebra de Lie é chamada nilpotente se na seqüência de ideais

$$
A_{(0)}=A, A_{(1)}=\left[A_{(0)}, A\right], \ldots, A_{(n)}=\left[A_{(n-1)}, A\right]
$$

para algum $n$ inteiro positivo, $A_{(n)}=0$.

Apresentaremos agora um resultado ${ }^{17}$ importante para a classificação das álgebras de Lie, e veremos que a estrutura dessas álgebras é passiva de análise, o que facilita a procura por uma determinada simetria.

Teorema 2 (Levi-Malcev) Qualquer álgebra de Lie $A$ pode ser escrita como uma soma semi-direta

$$
A=N \oplus_{s} S,
$$

de uma álgebra de Lie solúvel $N$ e uma álgebra de Lie semi-simples $S$.

Este teorema implica em 
$[N, N] \subset N, \quad[S, S] \subset S, \quad[N, S] \subset N$,

isto é, qualquer álgebra de Lie $A$ é uma soma semi-direta $N \oplus_{S} S$, de um ideal solúvel maximal $N$ e uma subálgebra semisimples de $A,{ }_{s} S$. Um exemplo vem dos geradores $\{p, R\}$, onde $\left\{p /\right.$ são geradores das translações e $\mathrm{R}$ são geradores das rotações, do grupo euclidiano $\mathrm{E}_{3}$, que satisfazem as relações

$$
[p, p] \subset p, \quad[R, R] \subset R, \quad[p, R] \subset p,
$$

e portanto temos a decomposição

$$
\mathrm{E}_{3}=T \oplus_{s} R
$$

\section{8 Álgebras de Lie Simples e Semi-Simples}

Uma álgebra de Lie $A$ é dita ser simples se ela não contem um ideal alem de A e $\{0\}$, enquanto que uma álgebra é dita ser semi-simples se não contêm ideal Abeliano. Uma álgebra simples é necessariamente semi-simples, embora o inverso não precisa ocorrer. A concepção de uma álgebra de Lie semi-simples atinge significância através do seguinte teorema ${ }^{17}$ :

Teorema 3 Uma álgebra de Lie $A$ é semi-simples se e somente se $A$ pode ser escrito como uma soma direta

$$
A=A_{1} \oplus \ldots \oplus A_{n},
$$

onde $A_{i}$ é um ideal de $A$, com cada ideal formando uma álgebra de Lie simples.

Este resultado é importante pois, dada uma álgebra de Lie arbitrária $A$, ele é útil para estabelecer critérios para classificar se $A$ é semi-simples.

\subsection{Forma de Killing}

Associado a qualquer grupo de Lie ou álgebra de Lie existe um tensor simétrico definido em termos das constantes de estrutura

$$
g_{\sigma \lambda}=g_{\lambda \sigma}=\sum_{\rho} \sum_{\tau} c^{\tau}{ }_{\sigma \rho} c^{\rho}{ }_{\lambda \tau}
$$

que é conhecido como tensor métrico ou forma de Killing. Note que esta matriz é $n \times n$, onde $\mathrm{n}$ é a quantidade de elemento da álgebra. Cartan sugeriu um simples teste para decidir se uma álgebra de Lie é semi-simples. 
Teorema 4 Uma álgebra de Lie $A$ é semi-simples, se somente se, $\operatorname{Det}\left|g_{\alpha \beta}\right| \neq 0$.

Vamos demonstrar que se uma álgebra não é semi-simples seu determinante é necessariamente nulo. Para isso vamos mostrar que a existência de um ideal abeliano implica em que pelo menos uma linha da forma de Killing é identicamente nula.

A existência de um ideal Abeliano significa que existem $r$ geradores que comutam dentre os $n$ geradores da álgebra, isto significa que as constantes de estrutura tem a propriedade

$$
c_{\bar{\alpha} \bar{\beta}}^{\rho}=0 \quad \bar{\alpha}, \bar{\beta}=1, \ldots, r \quad \rho=1, \ldots, n,
$$

onde denotamos as variáveis que percorrem os geradores do ideal Abeliano com uma barra.

A existência do ideal também garante que

$$
c_{\alpha \bar{\beta}}^{\rho}=0 \quad \bar{\beta}<r \text { e } \rho>r .
$$

porque o comutador de um gerador do ideal com um gerador arbitrário pertence ao ideal.

A forma de Killing é

$$
g_{\alpha \beta}=\sum_{\rho \sigma} c_{\alpha \rho}^{\sigma} c_{\sigma \beta}^{\rho} \text {. }
$$

Seja $\bar{\alpha}$ a linha que será demonstrada nula e pertence ao ideal Abeliano, então

$$
g_{\bar{\alpha} \beta}=\sum_{\bar{\rho} \bar{\sigma}} c_{\bar{\alpha} \rho}^{\sigma} c_{\bar{\sigma} \beta}^{\bar{\rho}}
$$

e como $c \frac{\bar{\sigma}}{\alpha \rho}=0$ temos,

$g_{\bar{\alpha} \beta}=0, \bar{\alpha}=1, \ldots, r$ e $\rho$ arbitrário, como queríamos demonstrar. Assim uma álgebra simples tem determinante necessariamente nulo.

A condição de Cartan dada no teorema 4 é justamente a condição da inversa $g^{\sigma \lambda}$ de $g_{\sigma \lambda}$ existir, e portanto para álgebras semi-simples podemos escrever

$$
g^{\mu \sigma} g_{\sigma v}=\delta^{\mu}{ }_{v} .
$$

Consideremos como um primeiro exemplo a álgebra de Lie so(3) cujos geradores satisfazem as seguintes relações de comutação:

$\left[X_{1}, X_{2}\right]=X_{3}, \quad\left[X_{2}, X_{3}\right]=X_{1}, \quad\left[X_{3}, X_{1}\right]=X_{2}$,

usando a equação $g_{\sigma \lambda}=g_{\lambda \sigma}=\sum_{\rho} \sum_{\tau} c^{\tau}{ }_{\sigma \rho} c^{\rho}{ }_{\lambda \tau}$ calculamos os elementos

$g_{11}=c^{\tau}{ }_{1 \rho} c^{\rho}{ }_{1 \tau}=c^{3}{ }_{12} c^{2}{ }_{13}+c^{2}{ }_{13} c^{3}{ }_{12}=(1)(-1)+(-1)(1)=-2$

$g_{22}=c^{\tau}{ }_{2 \rho} c^{\rho_{2 \tau}}=c^{3}{ }_{21} c^{l}{ }_{23}+c^{l}{ }_{23} c^{3}{ }_{21}=(-1)(1)+(1)(-1)=-2$

$g_{33}=c^{\tau}{ }_{3 \rho} c^{\rho_{3 \tau}}=c^{2}{ }_{31} c^{l}{ }_{32}+c^{\prime}{ }_{32} c^{2}{ }_{31}=(1)(-1)+(-1)(1)=-2$ 
$g_{12}=c^{\tau}{ }_{1 \rho} c^{\rho}{ }_{2 \tau}=0$

$g_{13}=c^{\tau}{ }_{1 \rho} c^{\rho}{ }_{3 \tau}=0$

$g_{2 l}=c^{\tau}{ }_{2 \rho} c^{\rho}{ }_{1 \tau}=0$

$g_{23}=c^{\tau}{ }_{2 \rho} c^{\rho}{ }_{3 \tau}=0$

$g_{31}=c^{\tau}{ }_{3 \rho} c^{\rho}{ }_{1 \tau}=0$

$g_{32}=c^{\tau}{ }_{3 \rho} c^{\rho}{ }_{2 \tau}=0$.

Daí,

$$
g_{\sigma \lambda}=\left(\begin{array}{ccc}
-2 & 0 & 0 \\
0 & -2 & 0 \\
0 & 0 & -2
\end{array}\right) .
$$

Encontramos que $g_{\sigma \lambda}=-2 \delta_{\sigma \lambda}, \operatorname{logo}$ det $\left|g_{\sigma \lambda}\right| \neq 0$, portanto a álgebra so(3) é semi-simples e a forma de Killing é definida negativa.

Seguindo a mesma estratégia do exemplo acima calculamos agora a forma de Killing para a álgebra de Lie $s o(2,1)$ cujos geradores satisfazem as relações de comutação

$\left[X_{1}, X_{2}\right]=X_{3}, \quad\left[X_{2}, X_{3}\right]=-X_{1},\left[X_{3}, X_{1}\right]=X_{2}$,

e os elementos da forma de Killing são

$$
\begin{aligned}
& g_{11}=c^{\tau}{ }_{1 \rho} c^{\rho_{1 \tau}}=c^{3}{ }_{12} c^{2}{ }_{13}+c^{2}{ }_{13} c^{3}{ }_{12}=(1)(-1)+(-1)(1)=-2 \\
& g_{22}=c^{\tau}{ }_{2 \rho} c^{\rho}{ }_{2 \tau}=c^{3}{ }_{21} c^{1}{ }_{23}+c^{1}{ }_{23} c^{3}{ }_{21}=(-1)(-1)+(-1)(-1)=2 \\
& g_{33}=c^{\tau}{ }_{3 \rho} c^{\rho}{ }_{3 \tau}=c^{2}{ }_{31} c^{1}{ }_{32}+c^{l}{ }_{32} c^{2}{ }_{31}=(1)(1)+(1)(1)=2 . \\
& g_{12}=c^{\tau}{ }_{1 \rho} c^{\rho}{ }_{2 \tau}=0 \\
& g_{13}=c^{\tau}{ }_{1 \rho} c^{\rho_{3 \tau}}=0 \\
& g_{21}=c^{\tau}{ }_{2 \rho} c_{1 \tau}{ }_{1 \tau}=0 \\
& g_{23}=c^{\tau}{ }_{2 \rho} c^{\rho_{3 \tau}}=0 \\
& g_{31}=c^{\tau}{ }_{3 \rho} c^{\rho}{ }_{1 \tau}=0 \\
& g_{32}=c^{\tau}{ }_{3 \rho} c^{\rho_{2 \tau}}=0 .
\end{aligned}
$$

Então

$$
g_{\sigma \lambda}=\left(\begin{array}{ccc}
-2 & 0 & 0 \\
0 & 2 & 0 \\
0 & 0 & 2
\end{array}\right),
$$


é a matriz de Killing dessa álgebra. Logo, det $g_{\sigma \lambda}=-8$. Portanto, a álgebra $s o(2,1)$ também é semi-simples.

Calcularemos agora a Forma de Killing genérica para a álgebra gl(n). Para isso definiremos $e_{\mu v}, e_{\sigma /}$ como os geradores da álgebra $g l(n)$, a qual apresenta as relações de comutação

$$
\begin{gathered}
{\left[e_{\mu \nu}, e_{\sigma \gamma}\right]=\delta_{\sigma v} e_{\mu \gamma}-\delta_{\mu \gamma} e_{\sigma v}} \\
=c^{\alpha \beta}{ }_{\mu \nu, \sigma \gamma} e_{\alpha \beta},
\end{gathered}
$$

onde $c_{\mu v, \sigma \gamma}^{\alpha \beta}$ é a constante de estrutura dessa álgebra. Podemos escrever esta constante da seguinte forma

$$
c_{\mu \nu, \sigma \gamma}^{\alpha \beta} e_{\alpha \beta}=\delta_{\sigma \nu} \delta_{\mu}^{\alpha} \delta_{\nu}^{\beta}-\delta_{\mu \gamma} \delta_{\sigma}^{\alpha} \delta_{\nu}^{\beta} .
$$

Então temos que

$$
\left[e_{\mu \nu}, e_{\sigma \gamma}\right]=\left(\delta_{\sigma \nu} \delta_{\mu}^{\alpha} \delta_{\nu}^{\beta}-\delta_{\mu \gamma} \delta_{\sigma}^{\alpha} \delta_{\nu}^{\beta}\right) e_{\alpha \beta},
$$

ou seja,

$$
g_{\mu v, \lambda \rho}=c_{\mu \nu, \sigma \gamma}^{\alpha \beta} c_{\lambda \rho, \alpha \beta}^{\sigma \gamma}
$$

Portanto:

$$
\begin{aligned}
g_{\mu v, \lambda \rho} & =\left(\delta_{\sigma \nu} \delta_{\mu}^{\alpha} \delta_{\nu}^{\beta}-\delta_{\mu \gamma} \delta_{\sigma}^{\alpha} \delta_{v}^{\beta}\right) c_{\lambda \rho, \alpha \beta}^{\sigma \gamma} \\
& =\delta_{\sigma \nu} c_{\lambda \rho, \mu \gamma}^{\sigma \gamma} \delta_{\mu \gamma} c_{\lambda \rho, \sigma v}^{\sigma \gamma} \\
& =c_{\lambda \rho, \mu \gamma}^{\gamma}-c_{\lambda \rho, \sigma \nu}^{\sigma \mu} \\
& =\delta_{\mu \rho} \delta_{\lambda}^{\nu} \delta_{\gamma}^{\gamma}-\delta_{\gamma \lambda} \delta_{\mu}^{\nu} \delta_{\rho}^{\gamma}-\delta_{\sigma \rho} \delta_{\lambda}^{\sigma} \delta_{\nu}^{\mu}+\delta_{\lambda \nu} \delta_{\sigma}^{\sigma} \delta_{\rho}^{\mu} \\
& =\delta_{\mu \rho} \delta_{v \lambda} n-\delta_{\rho \lambda} \delta_{\mu \nu}-\delta_{\lambda \rho} \delta_{\mu \nu}+\delta_{\nu \lambda} \delta_{\mu \rho} n \\
& =2 n \delta_{\mu \rho} \delta_{v \lambda}-2 \delta_{\mu \nu} \delta_{\lambda \rho},
\end{aligned}
$$

que é a forma de Killing para a álgebra $g l(n)$.

No caso particular da álgebra de lie $g l(2)$, cujos geradores são $e_{11}, e_{22}, e_{12}, e_{21}$, usando a fórmula (3.11) temos

$$
g_{\mu \nu, \lambda \rho}=4 \delta_{\mu \rho} \delta_{v \lambda}-2 \delta_{\mu \nu} \delta_{\lambda \rho}
$$

$g_{11,11}=2, g_{11.22}=-2, g_{11.12}=0, g_{11,21}=0, g_{22,11}=-2, g_{22,22}=2, g_{22.12}=0, g_{22,21}=0$

$g_{12,11}=0, g_{12.22}=0, g_{12.12}=0, g_{12,21}=4, g_{21,11}=0, g_{21,22}=0, g_{21,12}=4, g_{2 l, 21}=0$,

ou seja, 


$$
g=\left(\begin{array}{cccc}
2 & -2 & 0 & 0 \\
-2 & 2 & 0 & 0 \\
0 & 0 & 0 & 4 \\
0 & 0 & 4 & 0
\end{array}\right)
$$

Neste caso observa-se que o determinante desta matriz é zero, portanto temos que a álgebra $g l(2)$, não é semi-simples.

Usando a forma geral de Killing para a álgebra $g l(n)$ calcularemos a Forma de Killing geral para a Álgebra $s l(n)$. Seja $A_{i i}=e_{i i}-e_{i+l, i+1}$ os geradores da álgebra $s l(n)$. A partir da fórmula (3.11), e da definição de transformação adjunta apresentada na seção 3.5 temos que:

$$
\operatorname{adX}(Y)=[X, Y] \text {, }
$$

em termos de coordenadas,

$$
(a d X(Y))^{i}=[X, Y]^{i}=c_{l k}^{i} x^{l} y^{k},
$$

isto é,

$$
(a d X)_{k}^{i}=c_{l k}^{i} x^{i} .
$$

Assim, definimos então o produto escalar

$$
(X, Y)=\operatorname{Tr}(a d X . a d Y),
$$

daí temos

$$
\begin{aligned}
(X, Y) & =g_{\mu v, \lambda \rho} x_{\mu v} y_{\lambda \rho} \\
& =2 n \operatorname{Tr}(X, Y)-2 \operatorname{Tr} X \operatorname{Tr} Y \quad, \quad \text { para } \mu=v, \lambda=\rho .
\end{aligned}
$$

O conjunto $s l(n)$, por definição consiste de geradores de $g l(n)$ satisfazendo a condição $\operatorname{Tr} X=0$, e ele é um ideal de $g l(n)$. Apresentaremos agora um lema importante que será usado nessa construção.

Lema Seja $N$ um ideal de uma álgebra de Lie $A$. Se $X$ e $Y \in N$ então

$$
(X, Y)_{N}=(X, Y)_{A} \text {, }
$$

isto é, o valor da forma de Killing em $N$ é o mesmo que em $A$.

Pelo lema acima e pela fórmula (3.12a) temos que a forma de Killing para a álgebra $s l(n)$ é

$$
(X, Y)_{s l(n)}=2 n \operatorname{Tr}(X, Y) \quad X, Y \in \operatorname{sl}(n),
$$

ou seja,

$$
g_{\mu v, \lambda \rho}=2 n \delta_{\mu \rho} \delta_{v \lambda}
$$


Particularizando a Forma de Killing para a álgebra $s l(2)$, cujos geradores são $A_{11}, A_{12}$ e $A_{21}$, é:

$g_{11,11}=4, g_{11,12}=0, g_{1,2 I}=0$

$g_{12,11}=0, g_{12.12}=0, g_{12,21}=4$

$g_{21,11}=0, \quad g_{21,12}=4, g_{21,21}=0$.

Logo temos que:

$$
g=\left(\begin{array}{lll}
4 & 0 & 0 \\
0 & 0 & 4 \\
0 & 4 & 0
\end{array}\right),
$$

portanto como $\operatorname{det}(g)=-64$ temos que esta álgebra é semi-simples.

\subsection{Os Operadores de Casimir}

Vamos construir agora operadores quadráticos para uma álgebra de Lie $A$, tal que esses operadores comutem com todos os geradores dessa álgebra. Definimos então a quantidade

$$
C=\sum_{\rho \sigma} g^{\rho \sigma} X_{\rho} X_{\sigma}
$$

onde os $X_{\tau}$ são os geradores da álgebra de Lie $A$. Então comutando $C$ com todos os geradores de $A$ temos

$$
\begin{aligned}
{\left[C, X_{\tau}\right] } & =\sum_{\rho \sigma}\left(g^{\rho \sigma}\left[X_{\rho}, X_{\tau}\right] X_{\sigma}+g^{\rho \sigma} X_{\rho}\left[X_{\sigma}, X_{\tau}\right]\right) \\
& =\sum_{\rho \sigma \lambda}\left(g^{\rho \sigma} c_{\rho \tau}^{\lambda} X_{\lambda} X_{\sigma}+g^{\rho \sigma} c_{\sigma \tau}^{\lambda} X_{\rho} X_{\lambda}\right) \\
& =\sum_{\rho \sigma \lambda} \sum_{k s}\left(g^{\rho \sigma} c_{\rho \tau}^{\lambda} g_{\lambda k} X^{k} g_{\sigma \sigma} X^{s}+g^{\rho \sigma} c_{\sigma \tau}^{\lambda} g_{\rho k} X^{k} g_{\lambda s} X^{s}\right) \\
& =\sum_{\rho \sigma \lambda} \sum_{k s}\left(g^{\rho \sigma} g_{\sigma \tau} c_{\rho \tau}^{\lambda} g_{\lambda k}+g^{\rho \sigma} g_{\rho k} c_{\sigma \tau}^{\lambda} g_{\lambda s}\right) X^{k} X^{s} \\
& =\sum_{k s}\left\{\sum_{\rho}\left(\delta_{s}^{\rho} c_{\rho \tau k}\right)+\sum_{\sigma}\left(\delta_{k}^{\sigma} c_{\sigma \tau}\right)\right] X^{k} X^{s} \\
& =\sum\left(c_{s \tau k}+c_{k \tau \sigma}\right) X^{k} X^{s} .
\end{aligned}
$$




$$
c_{s t t}=\sum_{\lambda} c_{s \tau}^{\lambda} g_{\lambda k}=\sum_{\lambda} \sum_{l \rho} c_{s \tau}^{\lambda} c_{\lambda \rho}^{l} c_{l k}^{\rho} .
$$

Aplicando a identidade de Jacobi temos:

$$
\begin{aligned}
\sum_{\lambda} c_{\lambda \rho}^{l} c_{s \tau}^{\lambda} & =\sum_{\lambda} c_{\rho \lambda}^{l} c_{\tau s}^{\lambda} \\
& =-\sum_{\lambda}\left(c_{\tau \lambda}^{l} c_{s \rho}^{\lambda}+c_{s \lambda}^{l} c_{s \rho}^{\lambda}\right) .
\end{aligned}
$$

Portanto

$$
\begin{aligned}
c_{s \tau k} & =-\sum_{\lambda l \rho}\left(c_{\tau \lambda}^{l} c_{s \rho}^{\lambda} c_{l k}^{\rho}+c_{s \lambda}^{l} c_{\rho \tau}^{\lambda} c_{l k}^{\rho}\right) \\
& =\sum_{\lambda l \rho}\left(c_{\tau \lambda}^{l} c_{\rho s}^{\lambda} c_{l k}^{\rho}+c_{s \lambda}^{l} c_{\tau \rho}^{\lambda} c_{l k}^{\rho}\right),
\end{aligned}
$$

fazendo:

$$
\begin{aligned}
& s \rightarrow \tau \\
& \tau \rightarrow k \Rightarrow c_{s \tau k} \rightarrow c_{\tau k s} \\
& k \rightarrow s,
\end{aligned}
$$

temos que

$$
\begin{aligned}
c_{\tau k s} & =\sum_{\lambda \rho l}\left(c_{k \lambda}^{l} c_{\rho \tau}^{\lambda} c_{l s}^{\rho}+c_{\tau \lambda}^{l} c_{k \rho}^{\lambda} c_{l s}^{\rho} 0=\right. \\
& =\sum_{\lambda \rho l}\left(c_{\lambda t}^{\lambda} c_{l s}^{\rho} c_{\lambda k}^{l}+c_{s l}^{\rho} c_{\tau \lambda}^{l} c_{\rho k}^{\lambda}\right)= \\
& =\sum_{\lambda \rho l}\left(c_{\tau \lambda}^{l} c_{\rho s}^{\lambda} c_{l k}^{\rho}+c_{s \lambda}^{l} c_{\tau \rho}^{\lambda} c_{l k}^{\rho}\right)=c_{s t k} .
\end{aligned}
$$

Temos que $c_{\text {๙sk }}$ é anti-simétrico, pois fazendo $\tau \rightarrow s$ e $s \rightarrow \tau$ temos

$$
\begin{aligned}
c_{\text {tzk }} & =\sum_{\lambda \rho l}\left(c_{s \lambda}^{l} c_{\rho \tau}^{\lambda} c_{l k}^{\rho}+c_{\tau \lambda}^{l} c_{s \rho}^{\lambda} c_{l k}^{\rho}\right)= \\
& =\sum_{\lambda \rho l}\left(-c_{s \lambda}^{l} c_{\tau \rho}^{\lambda} c_{l k}^{\rho}-c_{\tau \lambda}^{l} c_{\rho s}^{\lambda} c_{l k}^{\rho}\right)=-c_{s \tau t} .
\end{aligned}
$$

Daí

$$
C_{s \tau k}+C_{k \tau s}=C_{s \tau t}+C_{s k \tau}=C_{s \tau k}-C_{s \tau k}=0 .
$$

Portanto como $c_{\tau \delta k}$ é anti-simétrico temos que $\left[C, X_{\tau}\right]=0$, para todo gerador $X_{\tau}$ da álgebra $A$.

Dessa forma o operador $C$ tem a importante propriedade de comutar com todos os geradores de uma álgebra de Lie semi-simples. Ele é conhecido por operador de Casimir .

Como exemplo calcularemos o operador de Casimir para a álgebra $s l(3)$, cujos geradores são $A_{11}, A_{22}, A_{12}, A_{21}, A_{13}, A_{3 l}, A_{23}, A_{32}$, onde $A_{i i}=e_{i i}-e_{i+1, i+1}$ e $A_{i j}=e_{i j}$. O primeiro passo é calcular o tensor métrico para esta álgebra. Então da equação (3.12b) obtemos a seguinte matriz 


\begin{tabular}{|l|l|l|l|l|l|l|l|l|}
\hline & 11 & 22 & 12 & 21 & 13 & 31 & 23 & 32 \\
\hline 11 & 12 & -6 & 0 & 0 & 0 & 0 & 0 & 0 \\
\hline 22 & -6 & 12 & 0 & 0 & 0 & 0 & 0 & 0 \\
\hline 12 & 0 & 0 & 0 & 6 & 0 & 0 & 0 & 0 \\
\hline 21 & 0 & 0 & 6 & 0 & 0 & 0 & 0 & 0 \\
\hline 13 & 0 & 0 & 0 & 0 & 0 & 6 & 0 & 0 \\
\hline 31 & 0 & 0 & 0 & 0 & 6 & 0 & 0 & 0 \\
\hline 23 & 0 & 0 & 0 & 0 & 0 & 0 & 0 & 6 \\
\hline 32 & 0 & 0 & 0 & 0 & 0 & 0 & 6 & 0 \\
\hline
\end{tabular}

onde a primeira coluna e a primeira linha representam os geradores da álgebra e os demais elementos são os respectivos elementos de matriz do tensor métrico. Invertendo esta matriz obtemos a matriz

\begin{tabular}{|l|l|l|l|l|l|l|l|l|}
\hline & 11 & 22 & 12 & 21 & 13 & 31 & 23 & 32 \\
\hline 11 & $1 / 9$ & $1 / 18$ & 0 & 0 & 0 & 0 & 0 & 0 \\
\hline 22 & $1 / 18$ & $1 / 9$ & 0 & 0 & 0 & 0 & 0 & 0 \\
\hline 12 & 0 & 0 & 0 & $1 / 6$ & 0 & 0 & 0 & 0 \\
\hline 21 & 0 & 0 & $1 / 6$ & 0 & 0 & 0 & 0 & 0 \\
\hline 13 & 0 & 0 & 0 & 0 & 0 & $1 / 6$ & 0 & 0 \\
\hline 31 & 0 & 0 & 0 & 0 & $1 / 6$ & 0 & 0 & 0 \\
\hline 23 & 0 & 0 & 0 & 0 & 0 & 0 & 0 & $1 / 6$ \\
\hline 32 & 0 & 0 & 0 & 0 & 0 & 0 & $1 / 6$ & 0 \\
\hline
\end{tabular}

Agora usando os coeficientes desta matriz inversa, calculamos o operador de Casimir para essa álgebra usando a equação (3.13):

$C=\frac{1}{6} e_{12} e_{21}+\frac{1}{6} e_{21} e_{12}+\frac{1}{6} e_{13} e_{31}+\frac{1}{6} e_{31} e_{13}+\frac{1}{6} e_{23} e_{32}+\frac{1}{6} e_{32} e_{23}+\frac{1}{9} H_{1}^{2}+\frac{1}{9} H_{2}^{2}+\frac{1}{18} H_{1} H_{2}+\frac{1}{18} H_{2} H_{1}$ Simplificando temos 
$C=\frac{1}{6}\left(e_{12} e_{21}+e_{21} e_{12}+e_{13} e_{31}+e_{31} e_{13}+e_{23} e_{32}+e_{32} e_{23}+\frac{2}{3} H_{1}^{2}+\frac{2}{3} H_{2}^{2}+6 H_{1} H_{2}\right)$,

onde $H_{1}=e_{11}-e_{22}$ e $H_{2}=e_{22}-e_{33}$, são os geradores da subálgebra de Cartan.

Neste exemplo podemos observar que o operador de Casimir é um múltiplo do elemento identidade. Esse fato é afirmado pelo lema de $\operatorname{Schur}^{18}$, o qual garante que qualquer operador que comuta com todos os elementos da álgebra será um múltiplo da identidade.

Racah $^{19}$ sugeriu a generalização dos operadores de Casimir, considerando os operadores

$$
I_{n}=\sum_{\substack{\alpha_{n} \beta_{1} \\ n=1,2, \ldots m}} c_{\alpha_{1} \beta_{1}}^{\beta_{2}} c_{\alpha_{2} \beta_{2}}^{\beta_{3}} \ldots c_{\alpha_{n} \beta_{n}}^{\beta_{1}} X^{\alpha_{1}} X^{\alpha_{2}} \ldots X^{\alpha_{n}}
$$

o qual também tem a propriedade de comutar com todos os elementos da álgebra de Lie semisimples.

\subsection{Sistema de Raízes}

Killing e Cartan mostraram que a operação de adjunção permite uma classificação das álgebras de Lie. Como resultado, uma álgebra de Lie $A$, de rank $r$, sempre apresenta uma subálgebra comutante $H$, denominada subálgebra de Cartan, cujos geradores $H_{i}, i=1,2, \ldots, r$ podem ser diagonalizados simultaneamente. Os autovalores correspondentes a cada gerador $H_{i}$, mantendo o mesmo autovetor $E_{\alpha}$, formam vetores $\alpha$ os quais são denominados de raízes. Como a subálgebra de Cartan é abeliana, então as raízes associadas aos geradores $H_{i}$ são nulas. Todas as demais raízes são distintas e sempre aparecem aos pares, ou seja, para cada raiz $\alpha$ há uma raiz - $\alpha$ Há essencialmente duas formas canônicas de apresentar as constantes de estrutura de uma álgebra de Lie: uma é conhecida como forma canônica de Cartan-Weyl e a outra denominada de forma canônica de Chevalley. Essas formas canônicas estão intimamente relacionadas com as bases nas quais as raízes são escritas.

\subsubsection{Forma canônịca de Cartan-Weyl}

Sabemos que uma base para uma álgebra de Lie pode ser linearmente transformada em outras bases. Buscaremos então, uma forma padrão para os comutadores dos geradores $X_{\tau}$ de uma álgebra de Lie semi-simples. Consideremos então a equação 


$$
[A, X]=\rho X,
$$

onde $A$ e $X$ são combinações lineares arbitrárias dos geradores de uma álgebra de Lie, então suponhamos que $A$ seja uma combinação linear arbitraria dos $X_{\tau}$

$$
A=\sum_{\mu} a^{\mu} X_{\mu}
$$

e $X$ seja outra combinação linear tal que

$$
X=\sum_{v} b^{v} X_{v} .
$$

A equação (3.18) pode ser expandida, de maneira que

$$
\begin{aligned}
{\left[\sum_{\mu} a^{\mu} X_{\mu}, \sum v b^{\nu} X_{v}\right] } & =\sum_{\nu} \rho b^{v} X_{v} \\
\sum_{\mu \nu \sigma} a^{\mu} b^{\nu} C_{\mu \nu}^{\sigma} X_{\sigma} & =\sum_{\nu} \rho b^{v} X_{v} \\
\sum_{\mu \nu \sigma} a^{\mu} b^{v} C_{\mu \nu}^{\sigma} X_{\sigma} & =\sum_{\nu} \rho b^{v} \delta_{\sigma}^{v} X_{\sigma} \\
\sum_{\mu \nu \sigma}\left(a^{\mu} C_{\mu \nu}^{\sigma}-\rho \delta_{\sigma}^{v}\right) x^{\nu} & =0
\end{aligned}
$$

Esta equação tem a forma de uma equação de autovalores para uma matriz $M_{v}^{\sigma}=\sum_{\mu} a^{\mu} C_{\mu v}^{\sigma}$ onde devemos esperar degenerescência. O fato mais importante aqui é que podemos escolher $A$ de forma que o número de soluções distintas da equação (3.18) seja máximo e que somente o autovalor $\rho=0$ seja degenerado. Esta degenerescência é chamada de rank da álgebra. Denotamos de $H_{i}$ os elementos associados as raízes nulas da equação (3.21), a equação (3.18) fica

$$
\left[A, H_{i}\right]=0, \quad i=1,2, \ldots, l
$$

Multiplicando a equação (3.22) por $\lambda^{i}$ e somando temos

$$
\lambda^{i}\left[A, H_{i}\right]=0=\left[A, \lambda^{i} H_{i}\right]=0 .
$$

Logo, concluímos que

$$
A=\lambda^{i} H_{i} .
$$

A equação (3.18) para os elementos não associados com soluções nulas, é escrito na forma

$$
\left[A, E_{\alpha}\right]=\alpha E_{\alpha} .
$$

Esta separação dos elementos será útil na seqüência de propriedades que comentaremos agora. Primeiro observamos que os operadores $H_{i}$ formam uma subálgebra. Para verificar isto observamos na identidade de Jacobi 


$$
\begin{array}{r}
{\left[A,\left[H_{\alpha}, H_{\beta}\right]\right]+\left[H_{\alpha},\left[H_{\beta}, A\right]\right]+\left[H_{\beta}\left[A, H_{\alpha}\right]\right]=0} \\
{\left[A,\left[H_{\alpha}, H_{\beta}\right]\right]=0,}
\end{array}
$$

que o comutador de $H_{\alpha}$ com $H_{\beta}$ deve ser uma combinação linear de $H_{i}$, do contrário não comuta $\operatorname{com} A$

$$
\left[H_{\alpha}, H_{\beta}\right]=C_{\alpha \beta}^{\tau} H_{\tau} .
$$

O interessante é que esta subálgebra é abeliana, isto é, $C_{\alpha \beta}^{\mathfrak{\tau}} \equiv 0$. A comutatividade de $H_{i}$ é garantida pela escolha de $A$ como combinação linear que formeça um número máximo de soluções não nula da equação (3.18). De fato, suponhamos que $\left[H_{\alpha}, H_{\beta}\right]=C_{\alpha \beta}^{\tau} H_{\tau}$ seja verdade com $C_{\alpha \beta}^{\tau} \neq 0$ para algum $\alpha$ e $\beta$, então fazemos as seguintes combinações lineares

$$
\mathrm{A}=\alpha^{i} H_{i} \quad \text { e } \quad \bar{X}=\beta^{j} H_{j} .
$$

Acrescentemos em $A=\lambda^{i} H_{i}$, a quantidade $\varepsilon$ :

$$
A^{\prime}=A+\varepsilon \mathrm{A},
$$

tão pequena tal que os autovalores não nulos de (3.18) não se altere efetivamente, isto é, continuem diferentes de 0

$$
\left[A^{\prime}, X\right]=\left(\rho+\varepsilon \rho^{\prime}\right) X \quad \text { com }\left(\rho+\varepsilon \rho^{\prime}\right) \neq 0 .
$$

Note que $A^{\prime}$ continua sendo uma combinação de $H_{i}, \log \left[A^{\prime}, X\right] \sim X$.

Como os $H_{i}$ formam uma álgebra, deve existir pelo menos um $s$ não nulo tal que

$$
[\mathrm{A}, \bar{X}]=s \bar{X} \text {. }
$$

Obtemos então

$$
\begin{aligned}
{\left[A^{\prime}, \bar{X}\right] } & =[A, \bar{X}]+\varepsilon[\mathrm{A}, \bar{X}] \\
& =\varepsilon s \bar{X}
\end{aligned}
$$

ou seja, encontramos um $A^{\prime}$ que fornece mais raízes distintas que $A$, contrariando o teorema de Cartan.

Portanto os $H_{i}$ são geradores da álgebra, que comutam entre si e geram uma subálgebra abeliana denominada subálgebra de Cartan. Veremos agora as relaçōes de comutação dos dois tipos de geradores, $H_{i}$ e $E_{\alpha}$.

Considerando o comutador

$$
\begin{aligned}
{\left[A,\left[H_{i}, E_{\alpha}\right]\right] } & =\left[A, H_{i} E_{\alpha}\right]-\left[A, E_{\alpha} H_{i}\right] \\
& =\left[A, H_{i}\right] E_{\alpha}+H_{i}\left[A, E_{\alpha}\right]-\left[A, E_{\alpha}\right] H_{i}-E_{\alpha}\left[A, H_{i}\right]
\end{aligned}
$$




$$
=\alpha\left[H_{i}, E_{\alpha}\right]
$$

concluímos que se $E_{\alpha}$ é um autovetor associado ao autovalor $\alpha$, existe $l$ autovetores $\left[H_{i}, E_{\alpha}\right]$ com o mesmo autovalor $\alpha$, onde $l$ é o rank. Mas os $\alpha_{s}$ são não degenerados e portanto os autovetores $\left[H_{i}, E_{\alpha}\right]$ devem ser proporcionais a $E_{\infty}$ isto é

$$
\left[H_{i}, E_{\alpha}\right]=\alpha_{i} E_{\alpha}=\alpha_{i} \delta_{\alpha}^{\tau} E_{\tau},
$$

e como

$$
\left[H_{i}, E_{\alpha}\right]=c_{l \alpha}^{\tau} E_{\tau}
$$

relacionando as constantes de estrutura com os $\alpha_{i}$, temos

$$
c_{i \alpha}^{\tau}=\alpha_{i} \delta_{\alpha}^{\tau} .
$$

Finalmente das equações (3.22) e (3.25) concluímos que

$$
\left[A, E_{\alpha}\right]=\left[\lambda^{i} H_{i}, E_{\alpha}\right]=\lambda^{i} \alpha_{i} E_{\alpha}=\alpha E_{\alpha} \Rightarrow \lambda^{i} \alpha_{i}
$$

e reconhecemos os $\alpha_{l}^{\prime}$ 's como componentes de um vetor $\alpha=\left(\alpha_{1}, \alpha_{2}, \ldots, \alpha_{4}\right)$, em um espaço de dimensão $l$. O vetor $\alpha$ é chamado de raiz, associada ao elemento $E_{\alpha}$ da álgebra.

Vejamos agora as propriedades do comutador de dois geradores de fora da subálgebra de Cartan. Para isso usaremos a identidade de Jacobi

$$
\left[A,\left[E_{\alpha}, E_{\beta}\right]\right]+\left[E_{\alpha},\left[E_{\beta}, A\right]\right]+\left[E_{\beta},\left[A, E_{\alpha}\right]\right]=0
$$

que fornece

$$
\left[A,\left[E_{\alpha}, E_{\beta}\right]\right]=(\alpha+\beta)\left[E_{\alpha}, E_{\beta}\right] .
$$

Usando na equação acima a relação

$$
\left[E_{\alpha}, E_{\beta}\right]=c_{\alpha \beta}^{\tau} E_{\tau},
$$

obtemos considerando que $\alpha+\beta$ seja raiz também

$$
\begin{aligned}
{\left[A, c_{\alpha \beta}^{\tau} E_{\tau}\right] } & =(\alpha+\beta) c_{\alpha \beta}^{\tau} E_{\tau} \\
{\left[A, E_{\tau}\right] } & =(\alpha+\beta) E_{\tau} \Rightarrow(\alpha+\beta)=\tau .
\end{aligned}
$$

Se caso $\alpha+\beta$ nāo for raiz, a única solução da equação (3.32) é

$$
\left[E_{\alpha}, E_{\beta}\right]=0 \text {. }
$$

Então

$$
\begin{aligned}
& {\left[E_{\alpha}, E_{\beta}\right]=c_{\alpha \beta}^{\mathrm{\tau}} E_{\alpha+\beta} \quad \text { se } \alpha+\beta \text { for raiz; }} \\
& {\left[E_{\alpha}, E_{\beta}\right]=0 \quad \text { se } \alpha+\beta \text { não for raiz. }}
\end{aligned}
$$


Notemos que se $\alpha+\beta=0$, a equação (3.32) se anula

$$
\left[A,\left[E_{\alpha}, E_{-\alpha}\right]\right]=0 .
$$

Porém a equação (3.22) sugere que $\left[E_{\alpha}, E_{-\alpha}\right]$ seja uma combinação linear de $H_{i}$

$$
\left[E_{\alpha}, E_{-\alpha}\right]=c_{\alpha-\alpha}^{i} H_{i} .
$$

Escolhida uma normalização adequada de $E_{\alpha}$, pode-se colocar a equação (3.34) na forma

$$
\left[E_{\alpha}, E_{-\alpha}\right]=\alpha_{i} E_{i} \text {. }
$$

Agora podemos escrever uma forma padrão, conhecida como base de Cartan-Weyl ou base cartesiana, das relações de comutação para uma álgebra de Lie semi-simples:

$$
\begin{aligned}
& {\left[H_{i}, H_{k}\right]=0} \\
& {\left[H_{i}, E_{\alpha}\right]=\alpha_{i} E_{\alpha}} \\
& {\left[E_{\alpha}, E_{\beta}\right]=\left\{\begin{array}{cc}
c_{\alpha \beta}^{\alpha+\beta} E_{\alpha+\beta} & \text { se } \alpha+\beta \neq 0 \text { e se } \alpha+\beta \text { for raiz } \\
0 & \text { se } \alpha+\beta \text { não for raiz }
\end{array}\right.} \\
& {\left[E_{\infty} E_{-\alpha}\right]=\alpha_{i} H_{i}=\sum_{i=1}^{r} \alpha_{i} H_{i}, \text { ré o rank } .}
\end{aligned}
$$

Dois importantes teoremas² são:

Teorema 5 Para toda raiz não nula $\alpha$ de uma álgebra de Lie semi-simples existe uma raiz $-\alpha$.

Teorema 6 Se $\alpha$ e $\beta$ são raízes então $2(\alpha, \beta) /(\alpha, \alpha)$ é um inteiro e $\beta-2 \alpha(\alpha, \beta) /(\alpha, \alpha)$ é também uma raiz A notação ( , ) é usada para indicar o produto escalar.

Teorema 7 Se $\alpha$ é uma raiz, então os únicos múltiplos $\alpha$ são: $\pm \alpha$.

Faremos a verificação deste teorema considerando as raízes da álgebra $g l(3)$. Então, das relações de comutação dadas pelas matrizes de Wyel, temos:

$$
\alpha_{12}=\left(\begin{array}{c}
1 \\
-1 \\
0
\end{array}\right), \quad \alpha_{13}=\left(\begin{array}{c}
1 \\
0 \\
-1
\end{array}\right) \text { e } \alpha_{23}=\left(\begin{array}{c}
0 \\
1 \\
-1
\end{array}\right) \text {. }
$$

Assim, fazendo $\alpha_{12}=\alpha, \alpha_{23}=\beta$ temos;

$$
\begin{aligned}
& (\alpha, \alpha)=2 \\
& (\alpha, \beta)=-1 \\
& 2(\alpha, \beta) /(\alpha, \alpha)=-1 \\
& \beta-2 \alpha(\alpha, \beta) /(\alpha, \alpha)=\alpha_{23}=\alpha+\beta .
\end{aligned}
$$


Uma raiz é positiva se a primeira componente não nula for positiva e uma raiz é simples se não for uma combinação de duas raízes positivas. No exemplo anterior $\alpha$ e $\beta$ são raízes simples e $\alpha \beta$ e $\alpha+\beta$ são positivas.

\subsubsection{A matriz de Cartan}

Cartan e Dynkin trabalhavam com raízes numa base onde as componentes $(v)_{i}$ de uma raiz qualquer $v$ são as projeçōes

$$
(v)_{i}=\frac{2}{\alpha_{i}^{2}} \alpha_{i} \cdot v,
$$

sobre as raízes simples $\alpha_{i}, i \leq r$. Esta base de dimensão igual ao rank $r$, denominada de Dynkin, é não-ortogonal e denotada por parênteses ${ }^{6}$, ( ). Por exemplo, na álgebra $g l(2)$ temos $r=1 \mathrm{e}$, portanto, uma única raiz simples: $\alpha_{l}=[1,-1]$. Esta raiz na base Dynkin é escrita como $\alpha_{l}=$ (2). Consideremos agora a álgebra $g l(3)$ onde $r=2$ e as raízes simples são $\alpha_{1}=[1,-1,0]$ e $\alpha_{2}$ $=[0,1,-1]$, na base Dynkin, usando a equação (3.37), as raízes simples passam a ser escritas como $\alpha_{1}=(2,-1)$ e $\alpha_{2}=(-1,2)$, respectivamente. Podemos formar uma matriz agrupando as raízes simples na base Dynkin como vetores colunas

$$
A=(2), \quad A=\left(\begin{array}{cc}
2 & -1 \\
-1 & 2
\end{array}\right) \text {, }
$$

para a álgebra $g l(2)$ e $g l(3)$, respectivamente, conhecida por matriz de Cartan. Em geral as componentes $a_{i k}$ da matriz de Cartan são dadas por

$$
a_{i k}=\frac{2}{\alpha_{i}^{2}} \alpha_{i} \cdot \alpha_{k}, \quad i, k \leq r,
$$

onde $\alpha_{i}$ são as raízes simples. Isto significa que as colunas da matriz de Cartan contém as raízes simples na base Dynkin para uma dada álgebra de Lie. Como uma conseqüência da classificação das álgebras de Lie feita por Cartan, a matriz de Cartan é conhecida para todas álgebras de Lie semi-simples clássicas e excepcionais. A partir da matriz de Cartan, as raízes simples e todas as demais são determinadas na base Dynkin. A matriz de Cartan (3.38) relacionada às álgebras de Lie finitas e semi-simples tem as seguintes propriedades: 
1. $a_{i i}=2$

2. $a_{i k}=0 \Rightarrow a_{k i}=0, \quad i \neq k$

3. $a_{i k} \leq 0, \quad i \neq k$, e $a_{i k}$ são inteiros

4. todos os menores principais de $A$ são positivos.

\subsubsection{Forma canônica de Chevalley}

Outra forma interessante de escrever as relações de comutação é a forma canônica de Chevalley. Ela é obtida da forma canônica de Cartan-Weyl pela prescrição

$$
h_{k}=\frac{2}{\alpha_{k}^{2}} \alpha_{k} \cdot H=\frac{2}{\alpha_{k}^{2}} \sum\left(\alpha_{k}\right)_{i} H_{i}, \quad k \leq r,
$$

onde $\alpha_{k}$ são as raízes simples e $H_{i}$ são os geradores da subálgebra de Cartan na forma canônica de Cartan-Weyl. Os geradores $E_{\alpha}$ fora da subálgebra de Cartan devem ser normalizados

$$
e_{k}=a E_{\alpha_{k}}, \quad f_{k}=a E_{-\alpha_{k}},
$$

onde $a$ é uma constante real e $E_{\alpha_{k}}$ são os geradores associados às raízes simples na forma canônica de Cartan-Weyl, para que possam satisfazer

$$
\begin{aligned}
& {\left[h_{i}, e_{k}\right]=+a_{i k} e_{k}} \\
& {\left[h_{i}, f_{k}\right]=-a_{i k} f_{k}} \\
& {\left[e_{i}, f_{k}\right]=+\delta_{i k} h_{k},}
\end{aligned}
$$

onde $a_{i k}$ são os elementos da matriz de Cartan. Assim, a forma canônica de Chevalley está intimamente relacionada com raízes na base Dynkin. Notemos que os comutadores anteriores envolvem apenas os geradores da subálgebra de Cartan e aqueles associados às raízes simples. Um outro conjunto de comutadores é dado por

$$
\left[E_{\alpha}, E_{\beta}\right]= \pm(p+1) E_{\alpha+\beta},
$$

onde $p=-1$ se $\alpha+\beta$ for uma raiz, $p$ é o menor inteiro para que $\beta$ - p $\alpha$ seja também uma raiz.

Consideremos como exemplo a álgebra $\operatorname{sl}(3)$ cujas raízes simples são $\alpha_{1}=[1,-1,0]=$ $(2,-1)$ e $\alpha_{2}=[0,1,-1]=(-1,2)$. A matriz de Cartan correspondente é

$$
A=\left(\begin{array}{cc}
2 & -1 \\
-1 & 2
\end{array}\right)
$$

agora, usando a prescrição (3.39), temos os dois geradores de traço nulo, 


$$
h_{1}=H_{1}+H_{2}=A_{11}-A_{22}, \quad h_{2}=H_{2}-H_{3}=A_{22}-A_{33},
$$

formando a subálgebra de Cartan da álgebra $s l(3)$. Os geradores associados às raízes simples são

$$
e_{1}=E_{\alpha_{1}}=A_{12}, \quad f_{1}=E_{-\alpha_{1}}=A_{21}, \quad e_{2}=E_{\alpha_{2}}=A_{23}, \quad f_{2}=E_{-\alpha_{2}}=A_{32},
$$

os geradores $A_{i k}$ são as matrizes de Weyl (3.3). Agora com esses elementos podemos escrever todas as relações de comutação,

$$
\begin{array}{lll}
{\left[h_{1}, e_{1}\right]=2 e_{1}} & {\left[h_{1}, f_{1}\right]=-2 f_{1}} & {\left[e_{1}, f_{1}\right]=h_{1}} \\
{\left[h_{1}, e_{2}\right]=-e_{2}} & {\left[h_{1}, f_{2}\right]=f_{2}} & {\left[e_{2}, f_{2}\right]=h_{2}} \\
{\left[h_{2}, e_{1}\right]=-e_{1}} & {\left[h_{2}, f_{1}\right]=f_{1}} & {\left[e_{1}, f_{2}\right]=0} \\
{\left[h_{2}, e_{2}\right]=2 e_{2}} & {\left[h_{2}, f_{2}\right]=-2 f_{2}} & {\left[e_{2}, f_{1}\right]=0 .}
\end{array}
$$

Vimos nesta seção conceitos e resultados importantes, os quais serão úteis no desenvolver das próximas seções.

\subsection{Representações das Álgebras de Lie}

Nesta seção apresentaremos o conceito de representação de uma álgebra de Lie, o qual é de central importância na formulação matemática das simetrias.

Seja $A$ uma álgebra de Lie sobre um corpo $K$ e seja $H$ um espaço linear, uma representação de $A$ em $H$ é um homorfismo $X \rightarrow T(X)$ de $A$ sobre o conjunto de operadores lineares em $H$, isto é, para $X$ e $Y$ em $A$ e $\alpha$ e $\beta$ em $K$

$$
\begin{aligned}
& \alpha X+\beta Y \rightarrow \alpha T(X)+\beta T(Y), \\
& {[X, Y] \rightarrow[T(X), T(Y)] \equiv T(X) T(Y)-T(Y) T(X) .}
\end{aligned}
$$

Notemos que em virtude da equação (3.44) a Identidade de Jacobi é automaticamente satisfeita.

As matrizes de Weyl formam uma representação matricial (irredutível) de ordem mais baixa (irreps fundamentais).

\subsection{Pesos}

Sabemos escrever as relações de comutação para qualquer álgebra de Lie clássica, através do conceito de raízes. Conhecemos as matrizes definindo estas álgebras (irrep 
fundamental), mas podemos precisar representar uma álgebra de Lie por outras matrizes (irredutíveis) de dimensão diferente das que definem a álgebra. Veremos que o conceito de pesos é uma forma útil para classificarmos as irreps de uma álgebra de Lie.

Um peso é um vetor no espaço das raízes tal que suas componentes são os autovalores dos elementos auto-comutantes, isto é, dos geradores da subálgebra de Cartan, para uma dada representação. Denotaremos então por $R_{\varphi}$ o espaço no qual uma representação $\varphi$ opera e por $N(\varphi)$ a dimensão desse espaço. As matrizes de representação podem ser construídas satisfazendo as bases de Cartan-Weyl ou de Chevalley. Dessa forma para uma representação de dimensão $n$ de um grupo de rank $l$ temos um conjunto $H_{\alpha_{t}}(i=1,2, \ldots, l)$ composto de $l$ matrizes $n \times n$ auto comutantes com $\alpha_{i} \in \Pi$ e um conjunto adicional $E_{\alpha}$ de matrizes $n \times n$ com $\alpha \in \Sigma$. Desde que $l$ matrizes $H_{\alpha_{i}}$ são auto-comutantes podemos construir um conjunto de autovetores $/ u\rangle$ para as $l$ matrizes no espaço $R$ tal que

$$
H_{\alpha_{i}}|u\rangle=\Lambda_{i}|u\rangle(i=1, \ldots, l),
$$

onde os $\Lambda_{i}$ são as componentes de um vetor $\Lambda$ em um espaço de dimensão $l$, ou seja construímos o vetor

$$
\Lambda=\left(\Lambda_{l}, \Lambda_{2}, \ldots, \Lambda_{l}\right),
$$

e definimos $\Lambda$ como o peso (ou vetor peso) do estado $|u\rangle$.

Teorema $8 \mathrm{Se}|u\rangle$ é um vetor de peso $\Lambda$, então $E_{\beta}|u\rangle$, onde $\beta$ é uma raiz, tem peso $\Lambda+\beta$. De fato

$$
\begin{aligned}
H_{\alpha_{i}} E_{\beta}\left|\mathrm{u}_{\Lambda}\right\rangle & =\left(\left[H_{\alpha_{i}}, E_{\beta}\right]+E_{\beta} H_{\alpha_{i}}\right)\left|u_{\Lambda}\right\rangle \\
& =\left[2 \frac{\left(\alpha_{i}, \beta\right)}{\left(\alpha_{i}, \alpha_{i}\right)}+\Lambda_{\alpha_{i}}\right] E_{\beta}\left|u_{\Lambda}\right\rangle \\
& =\left(\Lambda_{\alpha_{i}}+\beta_{i}\right) E_{\beta}\left|u_{\Lambda}\right\rangle .
\end{aligned}
$$

escrevendo $H_{\alpha}=\left(H_{\alpha_{1}}, \ldots, H_{\alpha_{i}}\right)$, temos

$$
H_{\alpha} E_{\beta}\left|u_{\Lambda}\right\rangle=(\Lambda+\beta) E_{\beta}\left|u_{\Lambda}\right\rangle \text {. }
$$

percebemos aqui que existe uma correlação algébrica entre pesos e raízes. De fato, as raízes são os pesos da representação adjunta, a qual é irredutível e apenas as raízes nulas são degeneradas (igual ao rank da álgebra). 
Notemos ainda que o espaço das representações $R_{\varphi}$ pode ser decomposto em uma soma direta de subespaços de pesos $R_{\varphi}^{\Lambda}$ tal que

$$
R_{\varphi}=\sum_{\Lambda \in \Delta_{\varphi}} R_{\varphi}^{\Lambda}=R_{\varphi}^{\Lambda_{1}} \oplus \cdots \oplus R_{\varphi}^{\Lambda_{i}} \oplus \cdots R_{\varphi}^{\Lambda l},
$$

onde $\Delta_{\varphi}$ é o conjunto de todos os vetores de peso, e a i-ésima parcela $R_{\varphi}^{\Lambda_{1}}$ define um subespaço de $R_{\varphi}$ gerado por todos estados de peso $\Lambda^{i},\left|u_{\Lambda^{i}}\right\rangle$.Todo vetor de $R_{\varphi}^{\Lambda}$ é chamado um vetor de peso, e da equação (3.46) temos

$$
\begin{aligned}
E_{\beta}\left|\xi_{\Lambda}\right\rangle & =R_{\varphi}^{\Lambda+\beta} & & \text { se } \Lambda+\beta \in \Delta_{\varphi} \\
& =0 & & \text { se } \Lambda+\beta \notin \Delta_{\varphi} .
\end{aligned}
$$

Um peso $\Lambda$ é positivo quando sua primeira componente não nula é positiva e um peso é maior que outro se a diferença entre eles é positiva. Um peso é simples se ele não for uma combinação linear de outros pesos positivos.

Teorema 9 Seja $\Lambda$ um peso e $\alpha$ uma raiz, então $n=2(\Lambda, \alpha) /(\alpha, \alpha)$ é um inteiro e $\Lambda-n \alpha$ é um peso.

Os teoremas seguintes classificam todas as representações irredutíveis:

Teorema 10 Toda representação $\varphi$ tem um peso máximo.

Teorema 11 Se a representação é irredutível, seu peso máximo é simples.

Teorema 12 Duas representações irredutíveis $\varphi_{1}$ e $\varphi_{2}$ são equivalentes se seus pesos máximos são iguais.

Teorema 13 Para $\Lambda$ ser o peso máximo de alguma representação $\varphi$ irredutível de $G$ é necessário e suficiente que todos os números

$$
\Lambda_{\alpha}=2 \frac{(\Lambda, \alpha)}{(\alpha, \alpha)} \quad(\alpha \in \Pi)
$$

sejam inteiros não negativos. 


\section{Capítulo 4}

\section{Determinação das Cadeias Sobreviventes}

Neste capítulo apresentaremos as estratégias e ferramentas usadas para determinar as cadeias sobreviventes, na procura por simetrias no código genético.

O primeiro passo na procura por simetrias no código genético consiste em selecionar uma álgebra de Lie simples $g$ e uma representação irredutível de $g$ em um espaço vetorial de dimensão 64, pois esta representação será atribuída ao espaço dos códons. A razão de escolher uma representação irredutível é que uma representação redutível é um objeto composto e pode ser expressada como a soma de componentes irredutíveis. O uso de representações redutíveis não corresponderia ao ponto de partida do processo de quebra de simetria, mas sim a um estágio posterior em que algum tipo de quebra já tenha ocorrido. Da mesma forma somente álgebras de Lie simples são consideradas, porque elas são os elementos fundamentais para a construção de álgebras de Lie semi-simples. Uma álgebra de Lie semi-simples é uma soma direta de álgebras de Lie simples e corresponderia a uma simetria composta.

\subsection{Representações do Espaço dos Códons}

A determinação de todas as representações, que podemos associar a representações de códons é baseada no celebre teorema da classificação de Cartan, o qual estabelece que as quatro álgebras de Lie clássicas, $\mathrm{A}_{r}=\mathrm{su}(r+1), \mathrm{B}_{r}=\mathrm{so}(2 r+1), \mathrm{C}_{r}=\mathrm{sp}(2 r)$ e $\mathrm{D}_{r}=\mathrm{so}(2 r)$, junto com as cinco álgebras de Lie excepcionais $E_{6}, E_{7}, E_{8}, F_{4}$ e $G_{2}$, esgotam as classes de álgebras de Lie simples. Analisando tais álgebras, percebemos que somente as álgebras de Lie simples su(2), 
su(3), su(4), sp(4), sp(6), so(13), so(14), $G_{2}$ e, obviamente, su(64) e sp(64) admitem uma representação tipo códon.

O resultado dessa análise pode ficar mais claro se observarmos as tabelas de representações irredutíveis das álgebras de Lie clássicas e excepcionais. Listamos na tabela 2 todas as representações irredutíveis das álgebras de Lie clássicas e excepcionais, em termos de seus pesos máximos, junto com suas dimensões d, até uma representação a mais que a de dimensão 64, de acordo com as tabelas de Mckay e Patera ${ }^{10}$.

$\mathrm{Na}$ tabela 2a note que a simetria $\mathrm{A}_{n}$, aparece em pares conjugados complexos, com pesos máximos $\left(a_{1}, a_{2}, \ldots, a_{r-1}, a_{r}\right)$ e $\left(a_{n}, a_{r-1}, \ldots, a_{2}, a_{l}\right)$, para simplificar somente uma das representações é mostrada, mesmo porque elas são equivalentes, exceto quando $a_{1}=a_{n} a_{2}=$ $a_{r-l}, \ldots$ Observa-se também que as dimensões das irreps (representações irredutíveis) crescem monotonicamente, ou seja, não ocilam, e portanto quando se chega a uma representação irredutível de dimensão > 64 sabemos que as demais terão dimensões mais altas. Verificamos que a representação fundamental da álgebra su(12) tem dimensão 12 e a adjunta tem dimensão 66, portanto as álgebras $\operatorname{su}(n)$ estão descartadas para $n>12$ exceto a su(64). Esse mesmo comportamento, alem do crescimento monótono, é observado nas tabelas $2 \mathrm{~b}, 2 \mathrm{c}$ e $2 \mathrm{~d}$, quando $n>15$ para as álgebras $\mathrm{B}_{n}$ e $\mathrm{D}_{n}$ e $n>12$ para as álgebras $\mathrm{C}_{n}$, respectivamente. Na tabela $2 \mathrm{e}$ observamos que as dimensões das irreps das álgebras excepcionais, $E_{6}, E_{7}, E_{8}, F_{4}$ e $G_{2}$, também crescem monotonicamente, e que com exceção de $\mathrm{G}_{2}$ não há irreps de dimensão 64 . 


\begin{tabular}{|c|c|}
\hline $\mathbf{A}_{\mathbf{2}}$ & su(3) \\
\hline Peso & $\mathrm{d}$ \\
Máximo & \\
\hline$(0,1)$ & 3 \\
$(0,2)$ & 6 \\
$(1,1)$ & 8 \\
$(0,3)$ & 10 \\
$(0,4)$ & 15 \\
$(1,2)$ & 15 \\
$(0,5)$ & 21 \\
$(1,3)$ & 24 \\
$(2,2)$ & 27 \\
$(0,6)$ & 28 \\
$(1,4)$ & 35 \\
$(0,7)$ & 36 \\
$(2,3)$ & 42 \\
$(0,8)$ & 45 \\
$(1,5)$ & 48 \\
$(0,9)$ & 55 \\
$(2,4)$ & 60 \\
$(1,6)$ & 63 \\
$(\mathbf{3 , 3})$ & $\mathbf{6 4}$ \\
$(0,10)$ & 66 \\
\hline
\end{tabular}

\begin{tabular}{|c|c|}
\hline $\mathbf{A}_{\mathbf{3}}-$ & su(4) \\
\hline Peso & $\mathrm{d}$ \\
Máximo & \\
\hline$(0,0,1)$ & 4 \\
$(0,1,0)$ & 6 \\
$(0,0,2)$ & 10 \\
$(1,0,1)$ & 15 \\
$(0,0,3)$ & 20 \\
$(0,1,1)$ & 20 \\
$(0,2,0)$ & 20 \\
$(0,0,4)$ & 35 \\
$(1,0,2)$ & 36 \\
$(0,1,2)$ & 45 \\
$(0,3,0)$ & 50 \\
$(0,0,5)$ & 56 \\
$(0,2,1)$ & 60 \\
$(\mathbf{1 , 1 , 1 )}$ & $\mathbf{6 4}$ \\
$(1,0,3)$ & 70 \\
& \\
& \\
& \\
\end{tabular}

\begin{tabular}{|c|c|}
\hline $\mathbf{A}_{4}$ & su(5) \\
\hline Peso & $\mathrm{d}$ \\
Máximo & \\
\hline$(0,0,0,1)$ & 5 \\
$(0,0,1,0)$ & 10 \\
$(0,0,0,2)$ & 15 \\
$(1,0,0,1)$ & 24 \\
$(0,0,0,3)$ & 35 \\
$(0,0,1,1)$ & 40 \\
$(0,1,0,1)$ & 45 \\
$(0,0,2,0)$ & 50 \\
$(0,0,0,4)$ & 70 \\
& \\
& \\
& \\
& \\
& \\
& \\
& \\
\end{tabular}

\begin{tabular}{|c|c|}
\hline $\mathbf{A}_{\mathbf{5}} \cdot$ & su(6) \\
\hline Peso & $\mathrm{d}$ \\
Máximo & \\
\hline$(0,0,0,0,1)$ & 6 \\
$(0,0,0,1,0)$ & 15 \\
$(0,0,1,0,0)$ & 20 \\
$(0,0,0,0,2)$ & 21 \\
$(1,0,0,0,1)$ & 35 \\
$(0,0,0,0,3)$ & 56 \\
$(0,0,0,1,1)$ & 70 \\
& \\
& \\
& \\
& \\
& \\
& \\
& \\
& \\
\end{tabular}

Tabela 2-a ${ }_{1}$ : Pesos máximos e dimensões das representações irredutíveis das álgebras de Lie clássicas $\mathrm{A}_{r}$ de posto baixo. 


\begin{tabular}{|c|c|}
\hline $\mathbf{A}_{\mathbf{6}}$ & - \\
\hline su(7) \\
\hline$(0,0,0,0,0,1)$ & 7 \\
$(0,0,0,0,1,0)$ & 21 \\
$(0,0,0,0,0,2)$ & 28 \\
$(0,0,0,1,0,0)$ & 35 \\
$(1,0,0,0,0,1)$ & 48 \\
$(0,0,0,0,0,3)$ & 84 \\
\hline
\end{tabular}

\begin{tabular}{|c|c|}
\hline $\mathbf{A}_{\mathbf{7}} \cdot \mathbf{s u}^{\mathbf{8}} \mathbf{8}$ \\
\hline Peso Máximo & $\mathrm{d}$ \\
\hline$(0,0,0,0,0,0,1)$ & 8 \\
$(0,0,0,0,0,1,0)$ & 28 \\
$(0,0,0,0,0,0,2)$ & 36 \\
$(0,0,0,0,1,0,0)$ & 56 \\
$(1,0,0,0,0,0,1)$ & 63 \\
$(0,0,0,1,0,0,0)$ & 70 \\
\hline
\end{tabular}

\begin{tabular}{|l|c|}
\hline $\mathbf{A}_{\mathbf{8}}$ & su(9) \\
\hline Peso Máximo & $\mathrm{d}$ \\
\hline$(0,0,0,0,0,0,0,1)$ & 9 \\
$(0,0,0,0,0,0,1,0)$ & 36 \\
$(0,0,0,0,0,0,0,2)$ & 45 \\
$(1,0,0,0,0,0,0,1)$ & 80 \\
& \\
\hline
\end{tabular}

\begin{tabular}{|c|c|}
\hline $\mathbf{A}_{\mathbf{9}}-$ & su(10) \\
\hline Peso Máximo & $\mathrm{d}$ \\
\hline$(0,0,0,0,0,0,0,0,1)$ & 10 \\
$(0,0,0,0,0,0,0,1,0)$ & 45 \\
$(0,0,0,0,0,0,0,0,2)$ & 55 \\
$(1,0,0,0,0,0,0,0,1)$ & 99 \\
\hline
\end{tabular}

\begin{tabular}{|c|c|}
\hline $\mathbf{A}_{\mathbf{1 0}}-$ & su(11) \\
\hline Peso Máximo & $\mathrm{d}$ \\
\hline$(0,0,0,0,0,0,0,0,0,1)$ & 11 \\
$(0,0,0,0,0,0,0,0,1,0)$ & 55 \\
$(0,0,0,0,0,0,0,0,0,2)$ & 66 \\
& \\
\hline
\end{tabular}

\begin{tabular}{|c|c|}
\hline $\mathbf{A}_{11}$ & $\mathbf{s u}(\mathbf{1 2})$ \\
\hline Peso Máximo & $\mathrm{d}$ \\
\hline$(0,0,0,0,0,0,0,0,0,0,1)$ & 12 \\
$(0,0,0,0,0,0,0,0,0,1,0)$ & 66 \\
\hline
\end{tabular}

Tabela 2-ą: Pesos máximos e dimensões das representações irredutíveis das álgebras de Lie clássicas $\mathrm{A}_{r}$ de posto baixo. 


\begin{tabular}{|c|c|}
\hline $\mathbf{B}_{\mathbf{3}}-$ & so(7) \\
\hline $\begin{array}{c}\text { Peso } \\
\text { Máximo }\end{array}$ & $\mathrm{d}$ \\
\hline$(1,0,0)$ & 7 \\
$(0,0,1)$ & 8 \\
$(0,1,0)$ & 21 \\
$(2,0,0)$ & 27 \\
$(0,0,2)$ & 35 \\
$(1,0,1)$ & 48 \\
$(3,0,0)$ & 77 \\
\hline
\end{tabular}

\begin{tabular}{|c|c|}
\hline $\mathbf{B}_{\mathbf{4}} \cdot$ & so(9) \\
\hline $\begin{array}{c}\text { Peso } \\
\text { Máximo }\end{array}$ & $\mathrm{d}$ \\
\hline$(1,0,0,0)$ & 9 \\
$(0,0,0,1)$ & 16 \\
$(0,1,0,0)$ & 36 \\
$(2,0,0,0)$ & 44 \\
$(0,0,1,0)$ & 84 \\
& \\
\hline
\end{tabular}

\begin{tabular}{|c|c|}
\hline $\mathbf{B}_{\mathbf{5}}$ & \multicolumn{1}{c|}{ so(11) } \\
\hline Peso & $\mathrm{d}$ \\
Máximo & \\
\hline$(1,0,0,0,0)$ & 11 \\
$(0,0,0,0,1)$ & 32 \\
$(0,1,0,0,0)$ & 55 \\
$(2,0,0,0,0)$ & 65 \\
& \\
& \\
\hline
\end{tabular}

\begin{tabular}{|c|c|}
\hline B $_{\mathbf{6}}$ & so(13) \\
\hline Peso Máximo & d \\
\hline$(1,0,0,0,0,0)$ & 13 \\
$(\mathbf{0 , 0 , 0 , 0 , 0 , 1 )}$ & $\mathbf{6 4}$ \\
$(0,1,0,0,0,0)$ & 78 \\
\hline
\end{tabular}

\begin{tabular}{|c|c|}
\hline $\mathbf{B}_{7}$ & so(15) \\
\hline Peso Máximo & $\mathrm{d}$ \\
\hline$(1,0,0,0,0,0,0)$ & 11 \\
$(0,1,0,0,0,0,0)$ & 105 \\
\hline
\end{tabular}

Tabela 2-b: Pesos máximos e dimensões das representações irredutíveis das álgebras de Lie clássicas $\mathrm{B}_{r}$ de posto baixo. 


\begin{tabular}{|c|c|}
\hline $\mathbf{C}_{\mathbf{2}}-$ & $\mathbf{s p}(\mathbf{4})$ \\
\hline $\begin{array}{c}\text { Peso } \\
\text { Máximo }\end{array}$ & $\mathrm{d}$ \\
\hline$(1,0)$ & 4 \\
$(0,1)$ & 5 \\
$(2,0)$ & 10 \\
$(0,2)$ & 14 \\
$(1,1)$ & 16 \\
$(3,0)$ & 20 \\
$(0,3)$ & 30 \\
$(2,1)$ & 35 \\
$(4,0)$ & 35 \\
$(1,2)$ & 40 \\
$(0,4)$ & 55 \\
$(5,0)$ & 56 \\
$(\mathbf{3 , 1}$ & $\mathbf{6 4}$ \\
$(1,3)$ & 80 \\
\hline
\end{tabular}

\begin{tabular}{|c|c|}
\hline \multicolumn{1}{|c|}{$\mathbf{C}_{\mathbf{3}}-$} & $\mathbf{s p}(\mathbf{6})$ \\
\hline Peso & $\mathrm{d}$ \\
Máximo & \\
\hline$(1,0,0)$ & 6 \\
$(0,1,0)$ & 14 \\
$(0,0,1)$ & 14 \\
$(2,0,0)$ & 21 \\
$(3,0,0)$ & 56 \\
$(\mathbf{1 , 1 , 0 )}$ & $\mathbf{6 4}$ \\
$(1,0,1)$ & 70 \\
& \\
& \\
& \\
& \\
\end{tabular}

\begin{tabular}{|c|c|}
\hline \multicolumn{1}{|c|}{$\mathbf{C}_{4}-$} & sp(8) \\
\hline Peso & $\mathrm{d}$ \\
Máximo & \\
\hline$(1,0,0,0)$ & 8 \\
$(0,1,0,0)$ & 27 \\
$(2,0,0,0)$ & 36 \\
$(0,0,0,1)$ & 42 \\
$(0,0,1,0)$ & 48 \\
$(3,0,0,0)$ & 120 \\
& \\
& \\
& \\
& \\
\end{tabular}

\begin{tabular}{|c|c|}
\hline $\mathbf{C}_{5}$ & sp $(\mathbf{1 0})$ \\
\hline Peso Máximo & $\mathrm{d}$ \\
\hline$(1,0,0,0,0)$ & 10 \\
$(0,1,0,0,0)$ & 44 \\
$(2,0,0,0,0)$ & 55 \\
$(0,0,1,0,0)$ & 110 \\
\hline
\end{tabular}

\begin{tabular}{|c|c|}
\hline $\mathbf{C}_{6}$ & $\mathbf{s p}(\mathbf{1 2})$ \\
\hline Peso Máximo & $\mathrm{d}$ \\
\hline$(1,0,0,0,0,0)$ & 12 \\
$(0,1,0,0,0,0)$ & 65 \\
& \\
& \\
\hline
\end{tabular}

Tabela 2-c: Pesos máximos e dimensões das representações irredutíveis das álgebras de Lie clássicas $\mathbf{C}_{r}$ de posto baixo. 


\begin{tabular}{|c|c|}
\hline $\mathbf{D}_{4} \quad-$ & so(8) \\
\hline Peso & $\mathrm{d}$ \\
Máximo & \\
\hline$(1,0,0,0)$ & 8 \\
$(0,0,1,0)$ & 8 \\
$(0,0,0,1)$ & 8 \\
$(0,1,0,0)$ & 28 \\
$(2,0,0,0)$ & 35 \\
$(0,0,2,0)$ & 35 \\
$(0,0,0,2)$ & 35 \\
$(1,0,1,0)$ & 56 \\
$(1,0,0,1)$ & 56 \\
$(0,0,1,1)$ & 56 \\
$(3,0,0,0)$ & 112 \\
$(0,0,3,0)$ & 112 \\
$(0,0,0,3)$ & 112 \\
\hline
\end{tabular}

\begin{tabular}{|c|c|}
\hline $\mathbf{D}_{\mathbf{5}}-$ & so(10) \\
\hline Peso & $\mathrm{d}$ \\
Máximo & \\
\hline$(1,0,0,0,0)$ & 10 \\
$(0,0,0,1,0)$ & 16 \\
$(0,0,0,0,1)$ & 16 \\
$(0,1,0,0,0)$ & 45 \\
$(2,0,0,0,0)$ & 54 \\
$(0,0,1,0,0)$ & 120 \\
& \\
& \\
& \\
& \\
& \\
\end{tabular}

\begin{tabular}{|c|c|}
\hline $\mathbf{D}_{\mathbf{6}}$ & \multicolumn{1}{c|}{ so(12) } \\
\hline Peso & $\mathrm{d}$ \\
Máximo & \\
\hline$(1,0,0,0,0,0)$ & 12 \\
$(1,0,0,0,1,0)$ & 32 \\
$(0,0,0,0,0,1)$ & 32 \\
$(0,1,0,0,0,0)$ & 66 \\
& \\
& \\
& \\
& \\
& \\
& \\
\end{tabular}

\begin{tabular}{|c|c|}
\hline $\mathbf{D}_{7} \quad$ - & so(14) \\
\hline Peso Máximo & d \\
\hline$(1,0,0,0,0,0,0)$ & 14 \\
$(\mathbf{0 , 0 , 0 , 0 , 0 , 1 , 0 )}$ & 64 \\
$(\mathbf{0 , 0 , 0 , 0 , 0 , 0 , 1 )}$ & 64 \\
$(0,1,0,0,0,0,0)$ & 91 \\
\hline
\end{tabular}

\begin{tabular}{|c|c|}
\hline $\mathbf{D}_{\mathbf{8}}$ & so(16) \\
\hline Peso Máximo & $\mathrm{d}$ \\
\hline$(1,0,0,0,0,0,0,0)$ & 16 \\
$(0,1,0,0,0,0,0,0)$ & 120 \\
& \\
\hline
\end{tabular}

Tabela 2-d: Pesos máximos e dimensões das representações irredutíveis das álgebras de Lie clássicas $\mathrm{D}_{r}$ de posto baixo. 


\begin{tabular}{|c|c|}
\hline \multicolumn{2}{|c|}{$\mathbf{E}_{6}$} \\
\hline Peso Máximo & d \\
\hline$(0,0,0,0,1,0)$ & 27 \\
$(0,0,0,0,0,1)$ & 78 \\
\hline
\end{tabular}

\begin{tabular}{|c|c|}
\hline \multicolumn{2}{|c|}{$\mathbf{E}_{7}$} \\
\hline Peso Máximo & $\mathrm{d}$ \\
\hline$(0,0,0,0,0,1,0)$ & 56 \\
$(1,0,0,0,0,0,0)$ & 133 \\
\hline
\end{tabular}

\begin{tabular}{|c|c|}
\hline \multicolumn{2}{|c|}{$\mathbf{E}_{\mathbf{8}}$} \\
\hline Peso Máximo & d \\
\hline$(1,0,0,0,0,0,0,0)$ & 248 \\
\hline
\end{tabular}

\begin{tabular}{|c|c|}
\hline \multicolumn{2}{|c|}{$\mathbf{F}_{4}$} \\
\hline Peso Máximo & $\mathrm{d}$ \\
\hline$(0,0,0,1)$ & 26 \\
$(1,0,0,0)$ & 52 \\
$(0,0,1,0)$ & 273 \\
& \\
\hline
\end{tabular}

\begin{tabular}{|c|c|}
\hline \multicolumn{2}{|c|}{$\mathbf{G}_{\mathbf{2}}$} \\
\hline Peso Máximo & $\mathrm{d}$ \\
\hline$(0,1)$ & 7 \\
$(1,0)$ & 14 \\
$(0,2)$ & 27 \\
$(\mathbf{1 , 1})$ & $\mathbf{6 4}$ \\
$(0,3)$ & $\mathbf{7 7}$ \\
\hline
\end{tabular}

Tabela 2-e: Pesos máximos e dimensões das representações irredutíveis das álgebras de Lie excepcionais $\mathrm{E}_{6}, \mathrm{E}_{7}, \mathrm{E}_{8}, \mathrm{~F}_{4}$ e $\mathrm{G}_{2}$. 
Analisando a tabela 2, selecionamos as álgebras com representações irredutíveis de dimensão 64 e chegamos a tabela 3 como a completa lista de representações do tipo códon.

\begin{tabular}{|c|c|c|}
\hline Classificação de Cartan & Álgebra de lie Simples & Peso Máximo \\
\hline $\mathrm{A}_{1}$ & $\mathrm{su}(2)$ & 63 \\
\hline $\mathrm{A}_{2}$ & $\mathrm{su}(3)$ & $(3,3)$ \\
\hline $\mathrm{C}_{2}$ & $\mathrm{sp}(4)$ & $(3,1)$ \\
\hline $\mathrm{G}_{2}$ & & $(1,1)$ \\
\hline $\mathrm{A}_{3}$ & $\mathrm{su}(4)$ & $(1,1,1)$ \\
\hline $\mathrm{C}_{3}$ & $\mathrm{sp}(6)$ & $(1,1,0)$ \\
\hline $\mathrm{B}_{6}$ & $\mathrm{so}(13)$ & $(0,0,0,0,0,1)$ \\
\hline $\mathrm{D}_{7}$ & $\mathrm{so}(14)$ & $(0,0,0,0,0,0,1,0)$ \\
\cline { 2 - 3 } & & $(0,0,0,0,0,0,0,1)$ \\
\hline $\mathrm{C}_{32}$ & $\mathrm{sp}(64)$ & $(1,0, \ldots, 0)$ \\
\hline $\mathrm{D}_{32}$ & $\mathrm{so}(64)$ & $(1,0, \ldots, 0)$ \\
\hline $\mathrm{A}_{63}$ & $\mathrm{su}(64)$ & $(1,0, \ldots, 0)$ \\
\hline
\end{tabular}

Tabela 3: Representações do tipo códon de álgebras de Lie simples.

Concluímos esta seção observando que consideraremos somente as representações do tipo códon das álgebras de Lie de posto baixo, pois as de posto alto, su(64), so(64) e sp(64), listadas na tabela 3 , tem um número grande de geradores e um número enorme de possibilidades de cadeias e podem então reproduzir qualquer degenerescência, tal que um esquema de quebra de simetria baseado em uma dessas álgebras é desprovido de força de previsão. Por exemplo no caso do su(64), pode-se reproduzir qualquer distribuição de multipletos quebrando em subálgebras da forma $\mathrm{su}\left(n_{1}\right) \oplus \ldots \oplus \mathrm{su}\left(n_{k}\right)$ com $k$ igual ao número de multipletos e $n_{l}, \ldots, n_{k}$ (com $\left.n_{l}+\ldots+n_{k}=64\right)$ igual a multiplicidade deles.

\subsection{Quebra de Simetria por Cadeias de Subálgebras}

Começando por uma das representações do tipo códon listadas na tabela 2, o primeiro passo da análise consiste em estabelecer regras de ramificação de maneira que a álgebra de Lie simples original reduza para qualquer de suas subálgebras maximais semi-simples, as quais foram classificadas por Dynkin ${ }^{23}$. As subálgebras maximais das álgebras de Lie simples mostradas na tabela 2, estão na tabela 4. 


\begin{tabular}{|c|c|c|}
\hline $\begin{array}{c}\text { Classificação de } \\
\text { Cartan }\end{array}$ & Álgebras de Lie Simples & Subálgebras Maximais Semi-Simples \\
\hline $\mathrm{A}_{1}$ & $\mathrm{su}(2)$ & - \\
\hline $\mathrm{A}_{2}$ & $\mathrm{su}(3)$ & $\mathrm{su}(2), \mathrm{su}(2)$ \\
\hline $\mathrm{C}_{2}$ & $\mathrm{sp}(4)$ & $\mathrm{su}(2) \oplus \mathrm{su}(2), \mathrm{su}(2)$ \\
\hline $\mathrm{G}_{2}$ & $\mathrm{G}_{2}$ & $\mathrm{su}(3), \mathrm{su}(2) \oplus \mathrm{su}(2), \mathrm{su}(2)$ \\
\hline $\mathrm{A}_{3}$ & $\mathrm{su}(4)$ & $\mathrm{su}(3), \mathrm{sp}(4), \mathrm{su}(2) \oplus \mathrm{su}(2)$ \\
\hline $\mathrm{C}_{3}$ & $\mathrm{sp}(6)$ & $\begin{array}{l}\mathrm{sp}(4) \oplus \mathrm{su}(2), \mathrm{su}(3) \\
\mathrm{su}(2) \oplus \mathrm{su}(2), \mathrm{su}(2)\end{array}$ \\
\hline $\mathrm{B}_{6}$ & so(13) & $\begin{array}{c}\mathrm{so}(12), \mathrm{su}(4) \oplus \mathrm{so}(7), \mathrm{sp}(4) \oplus \mathrm{so}(8) \\
\mathrm{su}(2) \oplus \mathrm{so}(10) \\
\mathrm{su}(2) \oplus \mathrm{su}(2) \oplus \mathrm{so}(9), \mathrm{su}(2)\end{array}$ \\
\hline$D_{7}$ & so(14) & $\begin{array}{c}\mathrm{su}(4) \oplus \mathrm{so}(8), \mathrm{su}(2) \oplus \mathrm{su}(2) \oplus \mathrm{so}(10) \\
\mathrm{su}(7), \mathrm{so}(13), \mathrm{so}(2) \oplus \mathrm{so}(11) \\
\mathrm{so}(7) \oplus \mathrm{so}(7), \mathrm{sp}(4) \oplus \mathrm{so}(9) \\
\mathrm{sp}(6), \mathrm{sp}(4), \mathrm{G}_{2}\end{array}$ \\
\hline
\end{tabular}

Tabela 4: Subálgebras maximais semi-simples de algumas álgebras de Lie simples.

As regras de ramificação para representações irredutíveis sob redução de simetria são conhecidas, elas tem sido tema de longas e intensivas investigações por muitos autores, usando uma variedade de técnicas, e muitos dos resultados (para álgebras de Lie simples de posto $\leq 8$, que são as que nos interessam) estão sumarizados nas tabelas de Mckay e Patera ${ }^{10}$.

Este processo de redução de simetria para subálgebras maximais pode ser repetido e conduzido para desenvolver cadeias de subálgebras, onde cada qual é maximal na subálgebra anterior. Para todas essas cadeias, a distribuição resultante de multiplicidades deve ser comparada com o que observamos no código genético, sumarizado na tabela 5. 


\begin{tabular}{|c|c|c|}
\hline $\begin{array}{c}\text { Degenerescência } \\
\text { do multipleto }\end{array}$ & $\begin{array}{c}\text { Número de } \\
\text { Multipletos }\end{array}$ & Aminoácidos \\
\hline 6 & 3 & Arg, Leu, Ser \\
4 & 5 & Ala, Gli, Pro, Trn, Val \\
3 & 2 & Ile, Fim \\
2 & 9 & Asn, Asp, Cis, Gli, \\
& & Glu, His, Lis, Fen, Tir \\
1 & 2 & Met, Trp \\
\hline
\end{tabular}

Tabela 5: Dimensões e multiplicidades do código genético padrão.

Mais precisamente, a estratégia é proceder ao longo de cada cadeia passo a passo e analisar, após cada passo, se a degenerescência é no entanto compatível com a do código genético. Se não for, a cadeia é não-sobrevivente e pode simplesmente ser desconsiderada sem mais análises. Se ela for compatível com a degenerescência do código genético, a cadeia é classificada como sobrevivente (até o estagio considerado), o que significa que devemos prosseguir com a análise nos próximos passos de quebras de simetrias. É claro que o número de cadeias que devem ser consideradas é a priori muito grande, principalmente para as álgebras so(13) e so(14). Dessa forma foi importante formular critérios que identificassem cadeias nãosobreviventes, para poder reduzir significativamente o número de cadeias que tiveram que ser submetidas a uma análise mais cuidadosa.

Para começar, observe que quando qualquer um dos critérios abaixo for satisfeito, a quebra de simetria deve ser terminada no estagio considerado.

- Mais que 21 multipletos.

- Mais que dois singletos.

- Mais que quatro multipletos de dimensão impar.

- Número insuficiente de multipletos de multiplicidade $\geq 6$ ou $\geq 4$.

Estes resultados vem do fato que, como se procede ao longo de qualquer cadeia dada, o número total de multipletos, o número de singletos e o número de multipletos de dimensão impar nunca 
podem diminuir (um multipleto de dimensão impar sempre quebrará em outros multipletos de dimensão impar). Similarmente o ultimo critério expressa a necessidade de existirem subespaços com dimensões suficientemente altas para conseguirmos através de quebras posteriores os três sextupletos e os cinco quadrupletos observados no código genético.

Por outro lado, é claro que quando um dos critérios abaixo é satisfeito a quebra de simetria não deve ser terminada mas sim proceder até o próximo estágio.

- Menos que 21 multipletos.

- Existência de multipletos de multiplicidade $\geq 7$.

- Existência de multipletos de multiplicidade 5.

- Mais que três multipletos de multiplicidade 6.

É o conflito entre um critério da primeira lista e um da segunda lista que nos possibilita classificar muitas cadeias como não-sobreviventes.

Um outro critério muito usado para classificar cadeias como não-sobreviventes é o

- Emparelhamento total.

Isto significa que o processo de redução levou a uma situação em que todas as representações irredutíveis da subálgebra considerada aparecem em pares conjugados ou, no caso de representações auto-conjugadas, com multiplicidade par. É claro que um tal emparelhamento de representações não pode ser removido por alguma quebra posterior. Isso leva a esquemas em que todas as multiplicidades são pares e é impossível obter três sextupletos, cinco quadrupletos ou nove dubletos, como são observados no código genético.

Formulamos então a procura por simetria no código genético, de acordo com a seguinte estratégia.

1. Seleciona-se uma das representações do tipo códon da tabela 2.

2. Submete-se esta representação a quebra de simetria através de todas as cadeias de subálgebras maximais possíveis, começando com uma das possibilidades listadas na tabela 3.

3. Após cada passo, analisa-se o reșultado e descartam-se todas as cadeias que são nãosobreviventes, de acordo com os critérios acima estabelecidos, antes de prosseguir para o próximo estágio. 
Ademais, é conveniente dividir este procedimento em duas fases.

Fase 1: Quebra da simetria primordial para a simetria su(2) ${ }^{p}$.

Durante a primeira fase, a quebra de simetria procede através de cadeias maximais de subálgebras semi-simples. Todas essas cadeias necessariamente terminarão em uma soma direta de $p$ cópias de su(2), a mais elementar de todas as álgebras de Lie simples, onde $p$ pode variar de 1 até o posto da álgebra de Lie original.

Fase 2: Quebra da simetria su(2)

A segunda fase consiste em quebrar uma ou várias das subálgebras su(2), usando operadores de quebra $\left(L_{z}\right.$ e $L_{z}^{2}$ ), depois da fase 1 ter sido completada.

\subsection{Quebra da Simetria Primordial para a Simetria su $(2)^{p}$}

Nesta seção mostraremos, através de exemplos, como é feita a quebra da simetria primordial para a simetria $\mathrm{su}(2)^{p}$, usando as regras de ramificação e identificando quais das cadeias são sobreviventes. Vamos começar considerando as cinco álgebras de Lie simples de posto baixo que admitem uma representaçāo do tipo códon, $\mathrm{su}(3), \mathrm{sp}(4), G_{2}$, su(4) e $\mathrm{sp}(6)$, para termos uma base de como procede essa análise. Depois mostraremos como proceder no caso das duas álgebras de Lie simples de posto médio, so(13) e so(14), que nos proporcionam centenas de cadeias, e que foi a parte crucial desta pesquisa.

As sub-representaçōes irredutíveis que aparecem no processo de redução serão tabeladas pelos seus pesos máximos, os quais no caso de $\operatorname{su}(2)$ são iguais a $2 s$, sendo $s$ o spin.

1. Cadeias de su(3): Existem duas cadeias possíveis:

- Cadeia 1: $\operatorname{su}(3) \supset \operatorname{su}(2)$

- Cadeia 2: su(3) つ so(3)

As regras de ramificação para a representação irredutível de su(3) de peso máximo $(3,3)$ levam às duas decomposiçōes mostradas na tabela 6 . Em ambos os casos, temos menos que 21 subespaços, de forma que a quebra de simetria deve continuar, mas as duas cadeias apresentam 8 subespaços de dimensão impar, logo nenhuma delas é sobrevivente. 


\begin{tabular}{|c|c|}
\hline \multicolumn{2}{|c|}{ su(2) } \\
\hline $2 \mathrm{~s}$ & $\mathrm{~d}$ \\
\hline 6 & 7 \\
\hline 5 & 6 \\
\hline 5 & 6 \\
\hline 4 & 5 \\
\hline 4 & 5 \\
\hline 4 & 5 \\
\hline 3 & 4 \\
\hline 3 & 4 \\
\hline 3 & 4 \\
\hline 3 & 4 \\
\hline 2 & 3 \\
\hline 2 & 3 \\
\hline 2 & 3 \\
\hline 1 & 2 \\
\hline 1 & 2 \\
\hline 0 & 1 \\
\hline
\end{tabular}

\begin{tabular}{|c|c|}
\hline \multicolumn{2}{|c|}{ so(3) } \\
\hline $2 \mathrm{~s}$ & $\mathrm{~d}$ \\
\hline 12 & 13 \\
\hline 10 & 11 \\
\hline 8 & 9 \\
\hline 8 & 9 \\
\hline 6 & 7 \\
\hline 6 & 7 \\
\hline 4 & 5 \\
\hline 2 & 3 \\
\hline
\end{tabular}

Tabela 6: Quebra da representação do tipo códon de su(3) nas cadeias não-sobreviventes $\operatorname{su}(3) \supset \operatorname{su}(2)$ e $\operatorname{su}(3) \supset \operatorname{so}(3)$.

2. Cadeias de sp(4): Novamente existe duas possíveis cadeias:

- Cadeia 1: $\operatorname{sp}(4) \supset \operatorname{su}(2)$

- Cadeia 2: $\operatorname{sp}(4) \supset \operatorname{su}(2) \oplus \operatorname{su}(2)$

As regras de ramificação aplicadas à representação irredutível de peso máximo $(3,1)$ de $s p(4)$ resultam em duas decomposições, as quais são mostradas na tabela 7. Em ambos os casos, temos somente 8 subespaços, não existem subespaços de dimensão impar, mas na cadeia 2 existem subespaços de dimensões suficientemente altas para gerar os 3 sextupletos e os 5 quadrupletos necessários para reproduzir a degenerescência do código genético, logo a cadeia 2 é sobrevivente e podemos prosseguir com a quebra de simetria, enquanto que a cadeia 1 é eliminada. 


\begin{tabular}{|c|c|}
\hline \multicolumn{2}{|c|}{$\mathbf{s u}(2)$} \\
\hline $2 \mathrm{~s}$ & $\mathrm{~d}$ \\
\hline 13 & 14 \\
\hline 11 & 12 \\
\hline 9 & 10 \\
\hline 7 & 8 \\
\hline 7 & 8 \\
\hline 5 & 6 \\
\hline 3 & 4 \\
\hline 1 & 2 \\
\hline
\end{tabular}

\begin{tabular}{|c|c|}
\hline \multicolumn{2}{|c|}{$\mathbf{s u}(2) \oplus \mathbf{s u}(2)$} \\
\hline$\left(2 \mathbf{s}_{1}, 2 \mathbf{s}_{2}\right)$ & $\mathrm{d}$ \\
\hline$(3,2)$ & 12 \\
\hline$(2,3)$ & 12 \\
\hline$(4,1)$ & 10 \\
\hline$(1,4)$ & 10 \\
\hline$(2,1)$ & 6 \\
\hline$(1,2)$ & 6 \\
\hline$(3,0)$ & 4 \\
\hline$(0,3)$ & 4 \\
\hline
\end{tabular}

Tabela 7: Quebra da representação do tipo códon de $\mathrm{sp}(4)$ na cadeia sobrevivente $\mathrm{sp}(4) \supset \mathrm{su}(2) \oplus \mathrm{su}(2)$ e na cadeia não-sobrevivente $\mathrm{sp}(4) \supset \mathrm{su}(2)$.

3. Cadeias de $G_{2}$ : Existem agora 4 cadeias possíveis:

- Cadeia 1: $G_{2} \supset \mathrm{su}(2) \oplus \operatorname{su}(2)$

- Cadeia 2: $G_{2} \supset \operatorname{su}(3) \supset \operatorname{su}(2)$

- Cadeia 3: $G_{2} \supset \operatorname{su}(3) \supset \operatorname{so}(3)$

- Cadeia 4: $G_{2} \supset \operatorname{su}(2)$

As regras de ramificação para à representação irredutível de peso máximo $(1,1)$ de $\mathrm{G}_{2}$ sob redução para suas subálgebras maximais dá as três decomposições mostradas na tabela 8 . A ultima delas, a cadeia 4, é eḷiminada porque exibe 6 subespaços de dimensão impar, enquanto que a cadeia 2 e a cadeia 3 são eliminadas devido ao emparelhamento total desde o inicio do nível su(3). Dessa forma somente a cadeia 1 é sobrevivente. 


\begin{tabular}{|c|c|}
\hline \multicolumn{2}{|c|}{$\mathbf{s u}(\mathbf{2}) \oplus \mathbf{s u}(\mathbf{2})$} \\
\hline$\left(2 \mathrm{~s}_{1}, 2 \mathrm{~s}_{2}\right)$ & $\mathrm{d}$ \\
\hline$(2,4)$ & 15 \\
\hline$(1,5)$ & 12 \\
\hline$(2,2)$ & 9 \\
\hline$(3,1)$ & 8 \\
\hline$(1,3)$ & 8 \\
\hline$(0,4)$ & 5 \\
\hline$(1,1)$ & 4 \\
\hline$(0,2)$ & 3 \\
\hline
\end{tabular}

\begin{tabular}{|c|c|}
\hline \multicolumn{2}{|c|}{$\mathbf{s u ( 3 )}$} \\
\hline Peso Máximo & $\mathrm{d}$ \\
\hline$(2,1)$ & 15 \\
\hline$(1,2)$ & 15 \\
\hline$(1,1)$ & 8 \\
\hline$(1,1)$ & 8 \\
\hline$(2,0)$ & 6 \\
\hline$(0,2)$ & 6 \\
\hline$(1,0)$ & 3 \\
\hline$(0,1)$ & 3 \\
\hline
\end{tabular}

\begin{tabular}{|c|c|}
\hline \multicolumn{2}{|c|}{$\mathbf{s u ( 2 )}$} \\
\hline $2 \mathrm{~s}$ & $\mathrm{~d}$ \\
\hline 16 & 17 \\
\hline 14 & 15 \\
\hline 10 & 11 \\
\hline 8 & 9 \\
\hline 6 & 7 \\
\hline 4 & 5 \\
\hline
\end{tabular}

Tabela 8: Quebra da representação do tipo códon de $G_{2}$ na cadeia sobrevivente $G_{2} \supset \operatorname{su}(2) \oplus \operatorname{su}(2)$ e nas cadeias não-sobreviventes $G_{2} \supset \operatorname{su}(3)$ e $G_{2} \supset \operatorname{su}(2)$.

4. Cadeias de su(4): Existem 5 cadeias a serem consideradas:

- Cadeia 1: $\operatorname{su}(4) \supset \operatorname{su}(3) \supset \operatorname{su}(2)$

- Cadeia 2: $\operatorname{su}(4) \supset \operatorname{su}(3) \supset \operatorname{so}(3)$

- Cadeia 3: $\operatorname{su}(4) \supset \operatorname{sp}(4) \supset \operatorname{su}(2)$

- Cadeia 4: $\operatorname{su}(4) \supset \operatorname{sp}(4) \supset \operatorname{su}(2) \oplus \operatorname{su}(2)$

- Cadeia 5: $\operatorname{su}(4) \supset \operatorname{su}(2) \oplus \operatorname{su}(2)$

Usando as regras de ramificação para a representação irredutível de peso máximo $(1,1,1)$ de su(4), temos 5 cadeias, as quais são todas eliminadas: as duas primeiras devido ao emparelhamento total ao nível su(3) e as outras três devido ao aparecimento de menos que 21 subespaços (cadeia 3: 10, cadeia 4: 14, cadeia 5: 8) e mais que 4 subespaços de dimensão impar (cadeia 3: 10, cadeia 4:8, cadeia 5: 8).

5. Cadeias de $\mathrm{sp}(6)$ : Neste caso temos 6 cadeias:

- Cadeia 1: $\operatorname{sp}(6) \supset \operatorname{su}(3) \supset \operatorname{su}(2)$

- Cadeia 2: $\operatorname{sp}(6) \supset \operatorname{su}(3) \supset \mathrm{so}(3)$

- Cadeia 3: $\operatorname{sp}(6) \supset \mathrm{sp}(4) \oplus \mathrm{su}(2) \supset \mathrm{su}(2) \oplus \mathrm{su}(2)$ 
- Cadeia 4: $\mathrm{sp}(6) \supset \mathrm{sp}(4) \oplus \mathrm{su}(2) \supset \mathrm{su}(2) \oplus \mathrm{su}(2) \oplus \mathrm{su}(2)$

- Cadeia 5: $\operatorname{sp}(6) \supset \operatorname{su}(2)$

- Cadeia 6: $\mathrm{sp}(6) \supset \mathrm{su}(2) \oplus \mathrm{su}(2)$

Usando as regras de ramificação para a representação irredutível de peso máximo $(1,1,0)$ de $\mathrm{sp}(6)$, eliminamos as cadeias 1 e 2 devido ao emparelhamento total ao nível su(3). As cadeias 5 e 6 , mostradas nas tabelas 9-a, são não-sobreviventes por não apresentarem subespaços em número suficiente para gerar os tripletos e sextupletos do código genético, enquanto que as cadeias 3 e 4 mostradas na tabela 9-b são sobreviventes: elas tem todos os requisitos para reproduzirem a degenerescência do código genético proveniente do processo de quebra de simetria.

\begin{tabular}{|c|c|}
\hline \multicolumn{2}{|c|}{$\mathbf{s u}(2)$} \\
\hline $2 \mathrm{~s}$ & $\mathrm{~d}$ \\
\hline 13 & 14 \\
\hline 11 & 12 \\
\hline 9 & 10 \\
\hline 7 & 8 \\
\hline 7 & 8 \\
\hline 5 & 6 \\
\hline 3 & 4 \\
\hline 1 & 2 \\
\hline
\end{tabular}

\begin{tabular}{|c|c|}
\hline \multicolumn{2}{|c|}{$\mathbf{s u}(2) \oplus \mathbf{s u}(2)$} \\
\hline$\left(2 \mathbf{s}_{1}, 2 \mathbf{s}_{2}\right)$ & $\mathrm{d}$ \\
\hline$(3,4)$ & 20 \\
\hline$(1,6)$ & 14 \\
\hline$(3,2)$ & 12 \\
\hline$(1,4)$ & 10 \\
\hline$(1,2)$ & 6 \\
\hline$(1,0)$ & 2 \\
\hline
\end{tabular}

Tabela 9-a: Quebra da representação do tipo códon de $\mathrm{sp}(6)$ nas cadeias não-sobreviventes $\mathrm{sp}(6) \supset \mathrm{su}(2)$ e $\mathrm{sp}(6) \supset \mathrm{su}(2) \oplus \mathrm{su}(2)$. 


\begin{tabular}{|c|c|c|c|}
\hline \multicolumn{2}{|l|}{$\operatorname{sp}(4) \oplus \operatorname{su}(2)$} & \multicolumn{2}{|c|}{$\operatorname{su}(2) \oplus \operatorname{su}(2) \oplus \operatorname{su}(2)$} \\
\hline Peso Máximo & $d$ & $\left(2 \mathrm{~s}_{1}, 2 \mathrm{~s}_{2}, 2 \mathrm{~s}_{3}\right)$ & $\mathrm{d}$ \\
\hline \multirow[t]{3}{*}{$((2,0), 1)$} & 20 & $(1,1,1)$ & 8 \\
\hline & & $(2,0,1)$ & 6 \\
\hline & & $(0,2,1)$ & 6 \\
\hline \multirow[t]{4}{*}{$((1,1), 0)$} & 16 & $(2,1,0)$ & 6 \\
\hline & & $(1,2,0)$ & 6 \\
\hline & & $(1,0,0)$ & 2 \\
\hline & & $(0,1,0)$ & 2 \\
\hline \multirow[t]{2}{*}{$((1,0), 1)$} & 12 & $(1,0,2)$ & $\overline{6}$ \\
\hline & & $(0,1,2)$ & 6 \\
\hline \multirow[t]{2}{*}{$((0,1), 1)$} & 10 & $(1,1,1)$ & 8 \\
\hline & & $(0,0,1)$ & 2 \\
\hline \multirow[t]{2}{*}{$((1,0), 0)$} & 4 & $(1,0,0)$ & 2 \\
\hline & & $(0,1,0)$ & 2 \\
\hline$((0,0), 1)$ & 2 & $(0,0,1)$ & 2 \\
\hline
\end{tabular}

\begin{tabular}{|c|c|c|c|}
\hline \multicolumn{2}{|c|}{$\mathbf{s p}(4) \oplus \mathbf{s u}(2)$} & \multicolumn{2}{|c|}{$\mathbf{s u}(2) \oplus \mathbf{s u}(2)$} \\
\hline Peso Máximo & $\mathrm{d}$ & $2 \mathrm{~s}_{1}-2 \mathrm{~s}_{2}$ & $\mathrm{~d}$ \\
\hline$(2,0), 1$ & 20 & 6,1 & 14 \\
\cline { 3 - 4 } & & 2,1 & 6 \\
\hline$(1,1), 0$ & \multirow{2}{*}{16} & 7,0 & 8 \\
\cline { 3 - 4 } & & 5,0 & 6 \\
\cline { 3 - 4 } & & 1,0 & 2 \\
\hline$(1,0), 2$ & 12 & 3,2 & 12 \\
\hline$(0,1), 1$ & 10 & 4,1 & 10 \\
\hline$(1,0), 0$ & 4 & 3,0 & 4 \\
\hline$(0,0), 1$ & 2 & 0,1 & 2 \\
\hline
\end{tabular}

Tabela 9-b: Quebra da representação do tipo códon de $\mathrm{sp}(6)$ nas cadeias sobreviventes $\mathrm{sp}(6) \supset \mathrm{sp}(4) \oplus \mathrm{su}(2) \supset \mathrm{su}(2) \oplus \mathrm{su}(2) \oplus \mathrm{su}(2)$ e $\mathrm{sp}(6) \supset \mathrm{sp}(4) \oplus \mathrm{su}(2) \supset \mathrm{su}(2) \oplus \mathrm{su}(2)$. 


\subsection{Análise das Álgebras $B_{6}$ e $D_{7}$ na Fase 1}

$\mathrm{Na}$ seção anterior foi explicada toda a metodologia usada na procura por simetrias no código genético. Como um primeiro exemplo mostramos a análise das álgebras de Lie simples de posto baixo, $\mathrm{su}(3), \mathrm{sp}(4), G_{2}$, su(4) e $\mathrm{sp}(6)$, através das quais foi possível explicar como as cadeias de subálgebras de Lie, candidatas a reproduzirem as degenerescências do código genético, foram selecionadas e analisadas na fase 1 , ou seja a quebra até uma soma de $A_{1}$ 's, usando apenas as tabelas de Mckay e Patera ${ }^{10}$ e os critérios de eliminação. Usaremos agora os métodos e os critérios de eliminação já mencionados, para analisar as álgebras $\mathrm{B}_{6}$ (so(13)) e $\mathrm{D}_{7}$ (so(14)), cuja análise é muito mais complexa devido ao grande número de cadeias.

Podemos dizer que está foi a parte principal e a mais trabalhosa deste trabalho, pois para as álgebras de posto mais baixo, temos poucas cadeias, então a análise se torna fácil, mas quando se trata de álgebras de posto maior (como so(13) e so(14)), temos um número muito maior de cadeias a serem analisadas, daí a necessidade de estabelecer rigorosos critérios de eliminação que tornassem possível descartar as cadeias não-sobreviventes com alguma base teórica. Na fase 1 dessa análise, além dos critérios de eliminação, fizemos uma espécie de mapa das cadeias (figura 5), no qual listamos todas as decomposições das álgebras so(13) e so(14) até a subálgebra su(2), o que foi muito útil para nos organizarmos melhor. 


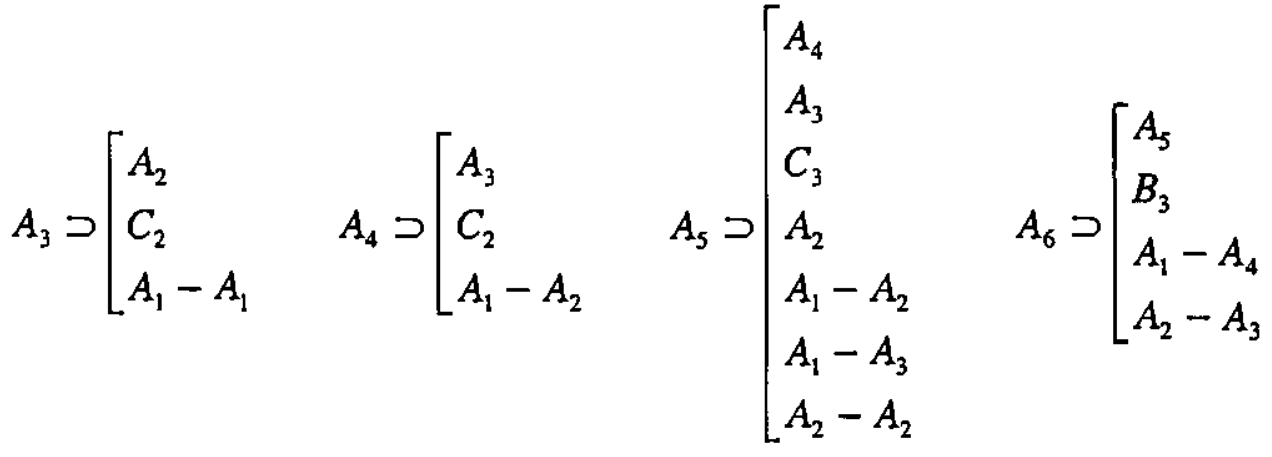

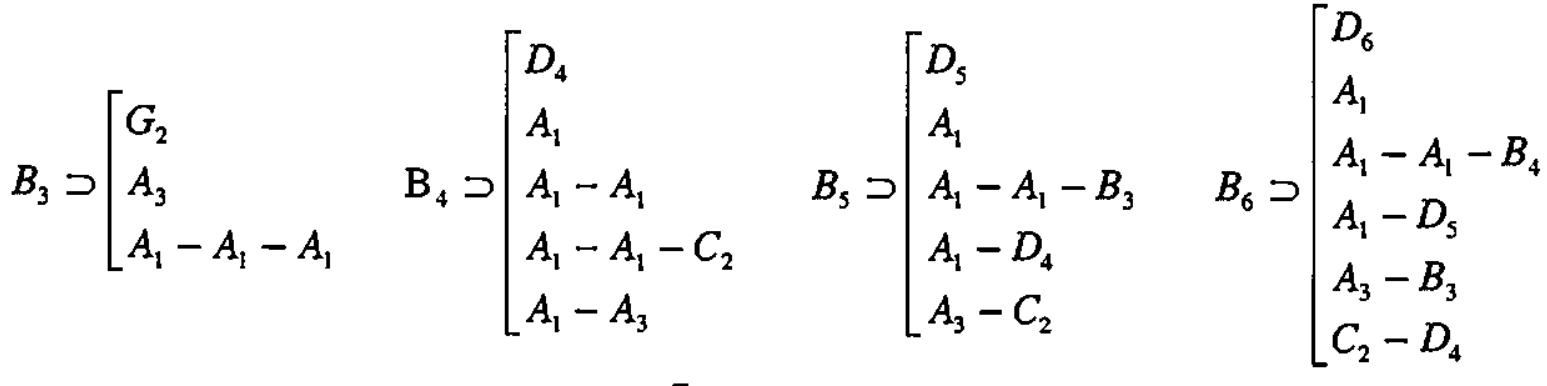

$$
\begin{aligned}
& C_{2} \supset\left[\begin{array}{l}
A_{1} \\
A_{1}-A_{1}
\end{array}\right. \\
& C_{3} \supset\left[\begin{array}{l}
A_{2} \\
A_{1} \\
A_{1}-A_{1} \\
A_{1}-C_{2}
\end{array}\right.
\end{aligned}
$$

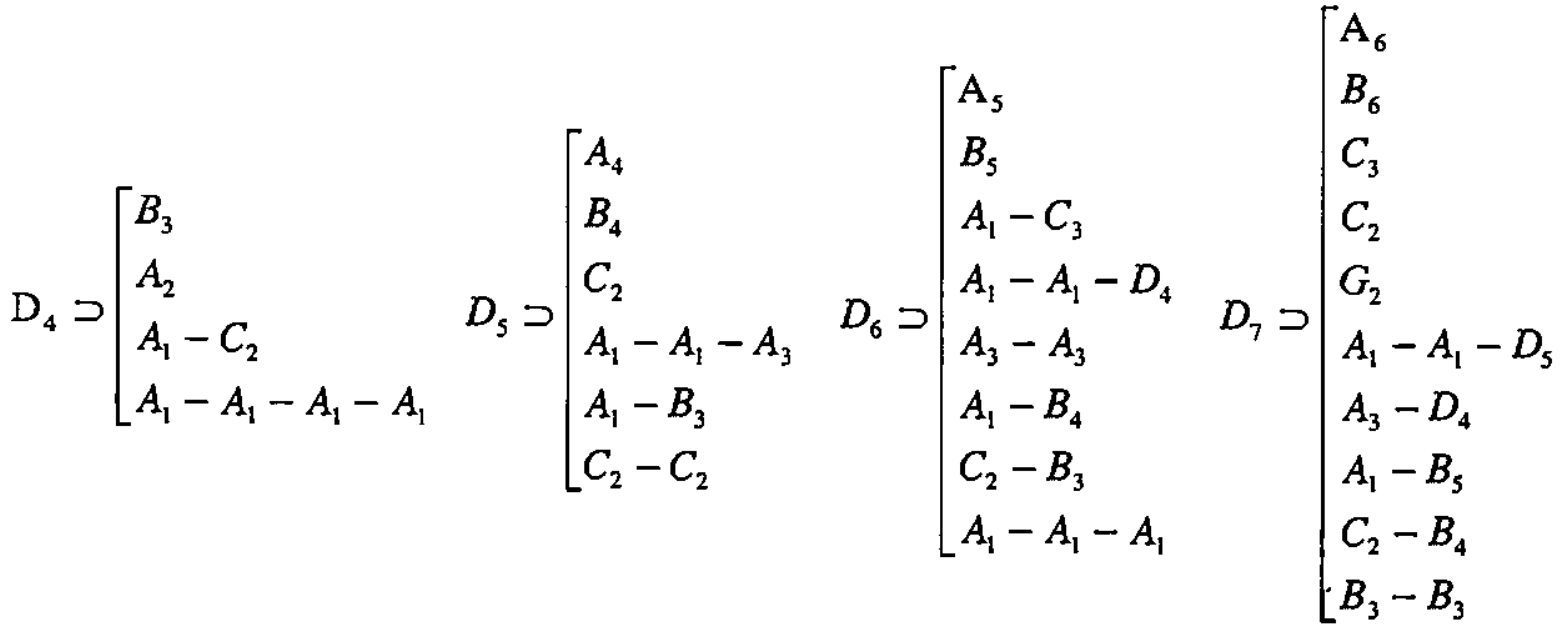$$
\mathrm{G}_{2} \supset\left[\begin{array}{l}
A_{2} \\
A_{1} \\
A_{1}-A_{1}
\end{array}\right.
$$

Figura 5: Mapa das Cadeias 


\section{Álgebra $B_{6}$}

A álgebra $\mathrm{B}_{6}$ como podemos observar acima no mapa das cadeias, quebra nas seguintes cadeias de subálgebras:

- Cadeia 1: $\mathrm{B}_{6} \supset \mathrm{D}_{6} \supset \ldots$

- Cadeia 2: $\mathrm{B}_{6} \supset \mathrm{A}_{1} \oplus \mathrm{A}_{1} \oplus \mathrm{B}_{4} \supset \ldots$

- Cadeia 3: $\mathrm{B}_{6} \supset \mathrm{A}_{1} \oplus \mathrm{D}_{5} \supset \ldots$

- Cadeia 4: $\mathrm{B}_{6} \supset \mathrm{A}_{3} \oplus \mathrm{B}_{3} \supset \ldots$

- Cadeia 5: $\mathrm{B}_{6} \supset \mathrm{C}_{2} \oplus \mathrm{D}_{4} \supset \ldots$

- Cadeia 6: $\mathrm{B}_{6} \supset \mathrm{A}_{1}$.

Continuando a quebra na fase 1, dividiremos agora as cadeias em sub-cadeias. A cadeia 1 é a maior delas e gera as seguintes sub-cadeias:

Sub-cadeias 1a: $\mathrm{B}_{6} \supset \mathrm{D}_{6} \supset \mathrm{A}_{5} \supset \ldots$

Sub-cadeias $1 \mathrm{~b}: \mathrm{B}_{6} \supset \mathrm{D}_{6} \supset \mathrm{B}_{5} \supset \ldots$

Sub-cadeias 1c: $\mathrm{B}_{6} \supset \mathrm{D}_{6} \supset \mathrm{A}_{1} \oplus \mathrm{C}_{3} \supset \ldots$

Sub-cadeias $1 \mathrm{~d}: \mathrm{B}_{6} \supset \mathrm{D}_{6} \supset \mathrm{A}_{1} \oplus \mathrm{A}_{1} \oplus \mathrm{D}_{4} \supset \ldots$

Sub-cadeias 1e: $\mathrm{B}_{6} \supset \mathrm{D}_{6} \supset \mathrm{A}_{3} \oplus \mathrm{A}_{3} \supset \ldots$

Sub-cadeias 1f: $\mathrm{B}_{6} \supset \mathrm{D}_{6} \supset \mathrm{A}_{1} \oplus \mathrm{B}_{4} \supset \ldots$

Sub-cadeias 1g: $B_{6} \supset D_{6} \supset C_{2} \oplus B_{3} \supset \ldots$

Sub-cadeia 1h: $\mathbf{B}_{6} \supset \mathbf{D}_{6} \supset \mathbf{A}_{1} \oplus \mathbf{A}_{1} \oplus \mathbf{A}_{1}$ 
Analisando as sub-cadeias 1a temos que elas são sobreviventes, então devemos prosseguir a quebra:

- $\mathrm{B}_{6} \supset \mathrm{D}_{6} \supset \mathrm{A}_{5} \supset \mathrm{A}_{4} \supset \ldots$

- $\mathrm{B}_{6} \supset \mathrm{D}_{6} \supset \mathrm{A}_{5} \supset \mathrm{A}_{3} \supset \ldots$

- $\mathrm{B}_{6} \supset \mathrm{D}_{6} \supset \mathrm{A}_{5} \supset \mathrm{C}_{3} \supset \ldots$

- $\mathrm{B}_{6} \supset \mathrm{D}_{6} \supset \mathrm{A}_{5} \supset \mathrm{A}_{2} \supset \ldots$

- $\mathrm{B}_{6} \supset \mathrm{D}_{6} \supset \mathrm{A}_{5} \supset \mathrm{A}_{1} \oplus \mathrm{A}_{2} \supset \ldots$

- $\mathrm{B}_{6} \supset \mathrm{D}_{6} \supset \mathrm{A}_{5} \supset \mathrm{A}_{1} \oplus \mathrm{A}_{3} \supset \ldots$

- $\mathrm{B}_{6} \supset \mathrm{D}_{6} \supset \mathrm{A}_{5} \supset \mathrm{A}_{2} \oplus \mathrm{A}_{2} \supset \ldots$

Observando as cadeias acima, não precisamos chegar até a soma de $A_{1}$ 's (su(2)), para classificá-las como não-sobreviventes, pois já neste estágio todas apresentam excesso de representações com dimensão 1 , logo todas são desclassificadas.

Nas sub-cadeias lb não precisamos dar mais nenhum passo no processo de quebra, pois já neste estágio verificamos que as representações aparecem emparelhadas e se prosseguirmos a quebra, o emparelhamento continuará, portanto podemos eliminá-las.

As sub-cadeias 1c são sobreviventes, então devemos prosseguir a quebra:

- $B_{6} \supset D_{6} \supset A_{1} \oplus C_{3} \supset A_{1} \oplus A_{1}$

- $\mathbf{B}_{6} \supset \mathbf{D}_{6} \supset A_{1} \oplus \mathrm{C}_{3} \supset A_{1} \oplus A_{1} \oplus A_{1}$

- $\mathrm{B}_{6} \supset \mathrm{D}_{6} \supset \mathrm{A}_{1} \oplus \mathrm{C}_{3} \supset \mathrm{A}_{1} \oplus \mathrm{A}_{2} \supset \mathrm{A}_{1} \oplus \mathrm{A}_{1}$

- $\mathrm{B}_{6} \supset \mathrm{D}_{6} \supset \mathrm{A}_{1} \oplus \mathrm{C}_{3} \supset \mathrm{A}_{1} \oplus \mathrm{A}_{1} \oplus \mathrm{C}_{2} \supset \mathrm{A}_{1} \oplus \mathrm{A}_{1} \oplus \mathrm{A}_{1}$

- $\mathrm{B}_{6} \supset \mathrm{D}_{6} \supset \mathrm{A}_{1} \oplus \mathrm{C}_{3} \supset \mathrm{A}_{1} \oplus \mathrm{A}_{1} \oplus \mathrm{C}_{2} \supset \mathrm{A}_{1} \oplus \mathrm{A}_{1} \oplus \mathrm{A}_{1} \oplus \mathrm{A}_{1}$

Analisando as cadeias acima verificamos que as duas primeiras são sobreviventes e devem ser submetidas à fase 2 , enquanto que as três ultimas são eliminadas pois a terceira cadeia 
apresenta excesso de representações de dimensão 1 , e as duas ultimas não contêm um número suficiente de representações de dimensão 6 para produzir os 3 sextupletos e os 2 tripletos.

Analisando as sub-cadeias 1d, verificamos que elas são sobreviventes e portanto devemos prosseguir a quebra:

- $\mathrm{B}_{6} \supset \mathrm{D}_{6} \supset \mathrm{A}_{1} \oplus \mathrm{A}_{1} \oplus \mathrm{D}_{4} \supset \mathrm{A}_{1} \oplus \mathrm{A}_{1} \oplus \mathrm{B}_{3}$

- $\mathrm{B}_{6} \supset \mathrm{D}_{6} \supset \mathrm{A}_{1} \oplus \mathrm{A}_{1} \oplus \mathrm{D}_{4} \supset \mathrm{A}_{1} \oplus \mathrm{A}_{1} \oplus \mathrm{A}_{2}$

- $\mathrm{B}_{6} \supset \mathrm{D}_{6} \supset \mathrm{A}_{1} \oplus \mathrm{A}_{1} \oplus \mathrm{D}_{4} \supset \mathrm{A}_{1} \oplus \mathrm{A}_{1} \oplus \mathrm{A}_{1} \oplus \mathrm{C}_{2} \supset \mathrm{A}_{1} \oplus \mathrm{A}_{1} \oplus \mathrm{A}_{1} \oplus \mathrm{A}_{1}$

- $\mathrm{B}_{6} \supset \mathrm{D}_{6} \supset \mathrm{A}_{1} \oplus \mathrm{A}_{1} \oplus \mathrm{D}_{4} \supset \mathrm{A}_{1} \oplus \mathrm{A}_{1} \oplus \mathrm{A}_{1} \oplus \mathrm{C}_{2} \supset \mathrm{A}_{1} \oplus \mathrm{A}_{1} \oplus \mathrm{A}_{1} \oplus \mathrm{A}_{1} \oplus \mathrm{A}_{1}$

- $\mathrm{B}_{6} \supset \mathrm{D}_{6} \supset \mathrm{A}_{1} \oplus \mathrm{A}_{1} \oplus \mathrm{D}_{4} \supset \mathrm{A}_{1} \oplus \mathrm{A}_{1} \oplus \mathrm{A}_{1} \oplus \mathrm{A}_{1} \oplus \mathrm{A}_{1} \oplus \mathrm{A}_{1}$

Analisando as cadeias acima verificamos que todas são eliminadas: as duas primeiras por emparelhamento, mesmo antes de atingir a soma de $A_{1}$ 's, e as três ultimas por falta de irreps de dimensão $\geq 6$ para produzir os três sextupletos necessários.

Nas sub-cadeias le, lf e lg, não precisamos dar mais nenhum passo no processo de quebra, pois já neste estágio verificamos que as representações aparecem emparelhadas e se prosseguirmos a quebra, o emparelhamento continuará, portanto podemos eliminá-las, enquanto que a sub-cadeia $1 \mathrm{~h}$ é sobrevivente e deve ser submetida à fase 2 .

Analisaremos agora a cadeia 2 que contêm as seguintes sub-cadeias:

Sub-cadeias 2a: $B_{6} \supset A_{1} \oplus A_{1} \oplus B_{4} \supset A_{1} \oplus A_{1} \oplus D_{4} \supset \ldots$

Sub-cadeias $2 b: B_{6} \supset A_{1} \oplus A_{1} \oplus B_{4} \supset A_{1} \oplus A_{1} \oplus A_{1} \oplus A_{3} \supset \ldots$

Sub-cadeias 2c: $\mathrm{B}_{6} \supset \mathrm{A}_{1} \oplus \mathrm{A}_{1} \oplus \mathrm{B}_{4} \supset \mathrm{A}_{1} \oplus \mathrm{A}_{1} \oplus \mathrm{A}_{1} \oplus \mathrm{A}_{1} \oplus \mathrm{C}_{2} \supset \ldots$

Sub-cadeia 2d: $B_{6} \supset A_{1} \oplus A_{1} \oplus B_{4} \supset A_{1} \oplus A_{1} \oplus A_{1} \oplus A_{1}$

Sub-cadeia 2e: $\mathrm{B}_{6} \supset \mathrm{A}_{1} \oplus \mathrm{A}_{1} \oplus \mathrm{B}_{4} \supset \mathrm{A}_{1} \oplus \mathrm{A}_{1} \oplus \mathrm{A}_{1}$ 
Verificando as cadeias acima podemos eliminar as sub-cadeias $2 \mathrm{~d}$ e $2 \mathrm{e}$, pois já chegaram à soma de $\mathrm{A}_{1}$ 's e não apresentam resultados satisfatórios (a sub-cadeia $2 \mathrm{~d}$ apresenta emparelhamento e a sub-cadeia 2e não reproduz os tripletos e sextupletos necessários).

Analisando as sub-cadeias 2a verificamos que elas são sobreviventes, então devemos prosseguir a quebra:

- $\mathrm{B}_{6} \supset \mathrm{A}_{1} \oplus \mathrm{A}_{1} \oplus \mathrm{B}_{4} \supset \mathrm{A}_{1} \oplus \mathrm{A}_{1} \oplus \mathrm{D}_{4} \supset \mathrm{A}_{1} \oplus \mathrm{A}_{1} \oplus \mathrm{B}_{3} \supset \ldots$

- $\mathrm{B}_{6} \supset \mathrm{A}_{1} \oplus \mathrm{A}_{1} \oplus \mathrm{B}_{4} \supset \mathrm{A}_{1} \oplus \mathrm{A}_{1} \oplus \mathrm{D}_{4} \supset \mathrm{A}_{1} \oplus \mathrm{A}_{1} \oplus \mathrm{A}_{2} \supset \ldots$

- $\mathrm{B}_{6} \supset \mathrm{A}_{1} \oplus \mathrm{A}_{1} \oplus \mathrm{B}_{4} \supset \mathrm{A}_{1} \oplus \mathrm{A}_{1} \oplus \mathrm{D}_{4} \supset \mathrm{A}_{1} \oplus \mathrm{A}_{1} \oplus \mathrm{A}_{1} \oplus \mathrm{C}_{2} \supset \mathrm{A}_{1} \oplus \mathrm{A}_{1} \oplus \mathrm{A}_{1} \oplus \mathrm{A}_{1}$

- $\mathrm{B}_{6} \supset \mathrm{A}_{1} \oplus \mathrm{A}_{1} \oplus \mathrm{B}_{4} \supset \mathrm{A}_{1} \oplus \mathrm{A}_{1} \oplus \mathrm{D}_{4} \supset \mathrm{A}_{1} \oplus \mathrm{A}_{1} \oplus \mathrm{A}_{1} \oplus \mathrm{C}_{2}$

$$
\supset A_{1} \oplus A_{1} \oplus A_{1} \oplus A_{1} \oplus A_{1}
$$

- $\mathrm{B}_{6} \supset \mathrm{A}_{1} \oplus \mathrm{A}_{1} \oplus \mathrm{B}_{4} \supset \mathrm{A}_{1} \oplus \mathrm{A}_{1} \oplus \mathrm{D}_{4} \supset \mathrm{A}_{1} \oplus \mathrm{A}_{1} \oplus \mathrm{A}_{1} \oplus \mathrm{A}_{1} \oplus \mathrm{A}_{1} \oplus \mathrm{A}_{1}$

Analisando as cadeias acima verificamos que todas são eliminadas, as duas primeiras por apresentar emparelhamento, mesmo antes de concluir a quebra até a soma de $\mathrm{A}_{1}$ 's e as três ultimas por falta de irreps que reproduzam subespaços de dimensão $\geq 6$.

As sub-cadeias $2 \mathrm{~b}$ são sobreviventes, então devemos prosseguir a quebra:

- $\mathrm{B}_{6} \supset \mathrm{A}_{1} \oplus \mathrm{A}_{1} \oplus \mathrm{B}_{4} \supset \mathrm{A}_{1} \oplus \mathrm{A}_{1} \oplus \mathrm{A}_{1} \oplus \mathrm{A}_{3} \supset \mathrm{A}_{1} \oplus \mathrm{A}_{1} \oplus \mathrm{A}_{1} \oplus \mathrm{A}_{2} \supset \ldots$

- $\mathrm{B}_{6} \supset \mathrm{A}_{1} \oplus \mathrm{A}_{1} \oplus \mathrm{B}_{4} \supset \mathrm{A}_{1} \oplus \mathrm{A}_{1} \oplus \mathrm{A}_{1} \oplus \mathrm{A}_{3} \supset \mathrm{A}_{1} \oplus \mathrm{A}_{1} \oplus \mathrm{A}_{1} \oplus \mathrm{C}_{2} \supset \ldots$

- $\mathrm{B}_{6} \supset \mathrm{A}_{1} \oplus \mathrm{A}_{1} \oplus \mathrm{B}_{4} \supset \mathrm{A}_{1} \oplus \mathrm{A}_{1} \oplus \mathrm{A}_{1} \oplus \mathrm{A}_{3} \supset \mathrm{A}_{1} \oplus \mathrm{A}_{1} \oplus \mathrm{A}_{1} \oplus \mathrm{A}_{1} \oplus \mathrm{A}_{1}$

As cadeias acima são eliminadas, até as duas primeiras que ainda não atingiram a soma de $A_{1}$ 's, pois todas apresentam emparelhamento.

As sub-cadeias $2 \mathrm{c}$ também são sobreviventes e devem ser quebradas novamente:

- $\mathrm{B}_{6} \supset \mathrm{A}_{1} \oplus \mathrm{A}_{1} \oplus \mathrm{B}_{4} \supset \mathrm{A}_{1} \oplus \mathrm{A}_{1} \oplus \mathrm{A}_{1} \oplus \mathrm{A}_{1} \oplus \mathrm{C}_{2} \supset \mathrm{A}_{1} \oplus \mathrm{A}_{1} \oplus \mathrm{A}_{1} \oplus \mathrm{A}_{1} \oplus \mathrm{A}_{1}$ 
- $\mathrm{B}_{6} \supset \mathrm{A}_{1} \oplus \mathrm{A}_{1} \oplus \mathrm{B}_{4} \supset \mathrm{A}_{1} \oplus \mathrm{A}_{1} \oplus \mathrm{A}_{1} \oplus \mathrm{A}_{1} \oplus \mathrm{C}_{2} \supset \mathrm{A}_{1} \oplus \mathrm{A}_{1} \oplus \mathrm{A}_{1} \oplus \mathrm{A}_{1} \oplus \mathrm{A}_{1} \oplus \mathrm{A}_{1}$

As duas cadeias acima devem ser eliminadas pois em nenhuma delas é possível obter os tripletos e os sextupletos necessários.

Analisando as cadeias 3 e 4, verificamos que elas apresentam emparelhamento no primeiro estágio da quebra, logo podemos classificá-las como não-sobreviventes, pois mesmo se prosseguirmos a quebra o emparelhamento se mantêm.

Analisaremos agora a cadeia 5, a qual é sobrevivente, e portanto precisamos continuar a quebra. Temos então as seguintes sub-cadeias:

Sub-cadeias 5a: $\mathrm{B}_{6} \supset \mathrm{C}_{2} \oplus \mathrm{D}_{4} \supset \mathrm{C}_{2} \oplus \mathrm{B}_{3} \supset \ldots$

Sub-cadeias 5b: $\mathrm{B}_{6} \supset \mathrm{C}_{2} \oplus \mathrm{D}_{4} \supset \mathrm{C}_{2} \oplus \mathrm{A}_{2} \supset \ldots$

Sub-cadeias 5c: $\mathrm{B}_{6} \supset \mathrm{C}_{2} \oplus \mathrm{D}_{4} \supset \mathrm{C}_{2} \oplus \mathrm{A}_{1} \oplus \mathrm{C}_{2} \supset \ldots$

Sub-cadeias 5d: $\mathrm{B}_{6} \supset \mathrm{C}_{2} \oplus \mathrm{D}_{4} \supset \mathrm{C}_{2} \oplus \mathrm{A}_{1} \oplus \mathrm{A}_{1} \oplus \mathrm{A}_{1} \oplus \mathrm{A}_{1} \supset \ldots$

As sub-cadeias $5 a$ e $5 b$ são eliminadas por emparelhamento. As sub-cadeias 5c são sobreviventes neste estágio, então devemos prosseguir a quebra:

- $\mathrm{B}_{6} \supset \mathrm{C}_{2} \oplus \mathrm{D}_{4} \supset \mathrm{C}_{2} \oplus \mathrm{A}_{1} \oplus \mathrm{C}_{2} \supset \mathrm{C}_{2} \oplus \mathrm{A}_{1} \oplus \mathrm{A}_{1} \supset \mathrm{A}_{1} \oplus \mathrm{A}_{1} \oplus \mathrm{A}_{1}$

- $\mathrm{B}_{6} \supset \mathrm{C}_{2} \oplus \mathrm{D}_{4} \supset \mathrm{C}_{2} \oplus \mathrm{A}_{1} \oplus \mathrm{C}_{2} \supset \mathrm{C}_{2} \oplus \mathrm{A}_{1} \oplus \mathrm{A}_{1} \supset \mathrm{A}_{1} \oplus \mathrm{A}_{1} \oplus \mathrm{A}_{1} \oplus \mathrm{A}_{1}$

- $\mathrm{B}_{6} \supset \mathrm{C}_{2} \oplus \mathrm{D}_{4} \supset \mathrm{C}_{2} \oplus \mathrm{A}_{1} \oplus \mathrm{C}_{2} \supset \mathrm{C}_{2} \oplus \mathrm{A}_{1} \oplus \mathrm{A}_{1} \oplus \mathrm{A}_{1} \supset \mathrm{A}_{1} \oplus \mathrm{A}_{1} \oplus \mathrm{A}_{1} \oplus \mathrm{A}_{\mathrm{I}}$

- $\mathrm{B}_{6} \supset \mathrm{C}_{2} \oplus \mathrm{D}_{4} \supset \mathrm{C}_{2} \oplus \mathrm{A}_{1} \oplus \mathrm{C}_{2} \supset \mathrm{C}_{2} \oplus \mathrm{A}_{1} \oplus \mathrm{A}_{1} \oplus \mathrm{A}_{1} \supset \mathrm{A}_{1} \oplus \mathrm{A}_{1} \oplus \mathrm{A}_{1} \oplus \mathrm{A}_{1} \oplus \mathrm{A}_{1}$

Analisando as cadeias acima verificamos que em todas elas não é possível obter os sextupletos e os tripletos necessários, portanto elas são eliminadas.

Finalizando, temos as sub-cadeias $5 d$ que são sobreviventes e, continuando a quebra, geram as seguintes cadeias:

- $\mathrm{B}_{6} \supset \mathrm{C}_{2} \oplus \mathrm{D}_{4} \supset \mathrm{C}_{2} \oplus \mathrm{A}_{1} \oplus \mathrm{A}_{1} \oplus \mathrm{A}_{1} \oplus \mathrm{A}_{1} \supset \mathrm{A}_{1} \oplus \mathrm{A}_{1} \oplus \mathrm{A}_{1} \oplus \mathrm{A}_{1} \oplus \mathrm{A}_{1}$ 
- $\mathrm{B}_{6} \supset \mathrm{C}_{2} \oplus \mathrm{D}_{4} \supset \mathrm{C}_{2} \oplus \mathrm{A}_{1} \oplus \mathrm{A}_{1} \oplus \mathrm{A}_{1} \oplus \mathrm{A}_{1} \supset \mathrm{A}_{1} \oplus \mathrm{A}_{1} \oplus \mathrm{A}_{1} \oplus \mathrm{A}_{1} \oplus \mathrm{A}_{1} \oplus \mathrm{A}_{1}$

Verificamos que em nenhuma das duas acima, é possível gerar os sextupletos e tripletos, logo são eliminadas.

A última das cadeias de subálgebras do $\mathrm{B}_{6}$, a cadeia 6 , também é eliminada pois possui 5 subespaços de dimensão impar. Com isso chegamos ao final da análise da álgebra $\mathrm{B}_{6}$, e dentre centenas de cadeias apenas 3 cadeias sobreviveram à fase $1 \mathrm{e}$ devem ser submetidas à fase 2 :

- $\mathrm{B}_{6} \supset \mathrm{D}_{6} \supset \mathrm{A}_{1} \oplus \mathrm{C}_{3} \supset \mathrm{A}_{1} \oplus \mathrm{A}_{1}$

- $\mathrm{B}_{6} \supset \mathrm{D}_{6} \supset \mathrm{A}_{1} \oplus \mathrm{A}_{1} \oplus \mathrm{A}_{1}$

- $\mathrm{B}_{6} \supset \mathrm{D}_{6} \supset \mathrm{A}_{1} \oplus \mathrm{C}_{3} \supset \mathrm{A}_{1} \oplus \mathrm{A}_{1} \oplus \mathrm{A}_{1}$

Observamos que a segunda e a terceira cadeia reproduzem as mesma degenerescências, devido a isso submeteremos apenas a primeira e segunda cadeia à fase 2, pois a ultima reproduziria o mesmo resultado. 


\section{Álgebra $\mathbf{D}_{7}$}

Analisaremos agora a álgebra $D_{7}$ na fase 1 . Com o auxilio do mapa das cadeias. listamos suas cadeias de subálgebras :

- Cadeia 1: $D_{7} \supset B_{6} \supset \ldots$

- Cadeia 2: $D_{7} \supset C_{3} \supset \ldots$

- Cadeia 3: $D_{7} \supset C_{2} \supset \ldots$

- Cadeia 4: $D_{7} \supset G_{2} \supset \ldots$

- Cadeia 5: $\mathrm{D}_{7} \supset \mathrm{A}_{6} \supset \ldots$

- Cadeia 6: $D_{7} \supset A_{1} \oplus A_{1} \oplus D_{5} \supset \ldots$

- Cadeia 7: $\mathrm{D}_{7} \supset \mathrm{A}_{3} \oplus \mathrm{D}_{4} \supset \ldots$

- Cadeia 8: $\mathrm{D}_{7} \supset \mathrm{A}_{1} \oplus \mathrm{B}_{5} \supset \ldots$

- Cadeia 9: $D_{7} \supset C_{2} \oplus B_{4} \supset \ldots$

- Cadeia 10: $D_{7} \supset B_{3} \oplus B_{3} \supset \ldots$

Como podemos ver as cadeias 1, 2, 3 e 4 já foram analisadas nas seções anteriores, então começamos pela cadeia 5 , que a princípio é sobrevivente, então devemos prosseguirmos a quebra, daí as seguintes sub-cadeias:

Sub-cadeias $5 \mathrm{a}: \mathrm{D}_{7} \supset \mathrm{A}_{6} \supset \mathrm{A}_{5} \supset \ldots$

Sub-cadeias 5b: $D_{7} \supset A_{6} \supset B_{3} \supset \ldots$

Sub-cadeias 5c: $D_{7} \supset A_{6} \supset A_{1} \oplus A_{4} \supset \ldots$

Sub-cadeias 5d: $D_{7} \supset A_{6} \supset A_{2} \oplus A_{3} \supset \ldots$

Analisando as sub-cadeias acima temos que elas são sobreviventes, então devemos prosseguir a quebra, em todas elas: 
Sub-cadeias 5a:

- $\mathrm{D}_{7} \supset \mathrm{A}_{6} \supset \mathrm{A}_{5} \supset \mathrm{A}_{4} \supset \ldots$

- $\mathrm{D}_{7} \supset \mathrm{A}_{6} \supset \mathrm{A}_{5} \supset \mathrm{A}_{3} \supset \ldots$

- $\mathrm{D}_{7} \supset \mathrm{A}_{6} \supset \mathrm{A}_{5} \supset \mathrm{C}_{3} \supset \ldots$

- $\mathrm{D}_{7} \supset \mathrm{A}_{6} \supset \mathrm{A}_{5} \supset \mathrm{A}_{2} \supset \ldots$

- $\mathrm{D}_{7} \supset \mathrm{A}_{6} \supset \mathrm{A}_{5} \supset \mathrm{A}_{1} \oplus \mathrm{A}_{2} \supset \ldots$

- $\mathrm{D}_{7} \supset \mathrm{A}_{6} \supset \mathrm{A}_{5} \supset \mathrm{A}_{1} \oplus \mathrm{A}_{3} \supset \ldots$

- $\mathrm{D}_{7} \supset \mathrm{A}_{6} \supset \mathrm{A}_{5} \supset \mathrm{A}_{2} \oplus \mathrm{A}_{2} \supset \ldots$

Verificando as cadeias acima temos que todas podem ser eliminadas mesmo antes da quebra atingir a soma de $A_{1}$ 's, a segunda e a quarta por emparelhamento $e$ as demais por apresentarem irreps com dimensão 1 em excesso, ou seja, mais de que duas.

Sub-cadeias 5b:

- $\mathrm{D}_{7} \supset \mathrm{A}_{6} \supset \mathrm{B}_{3} \supset \mathrm{G}_{2} \supset \ldots$

- $\mathrm{D}_{7} \supset \mathrm{A}_{6} \supset \mathrm{B}_{3} \supset \mathrm{A}_{3} \supset \ldots$

- $D_{7} \supset A_{6} \supset B_{3} \supset A_{1} \oplus A_{1} \oplus A_{1}$

As cadeias acima também são eliminadas, a primeira por emparelhamento, a segunda por apresentar 6 subespaços de dimensão impar e a terceira por apresentar 8 subespaços de dimensão impar.

Sub-cadeias 5c:

- $D_{7} \supset A_{6} \supset A_{1} \oplus A_{4} \supset A_{1} \oplus A_{3} \supset \ldots$

- $\mathrm{D}_{7} \supset \mathrm{A}_{6} \supset \mathrm{A}_{1} \oplus \mathrm{A}_{4} \supset \mathrm{A}_{1} \oplus \mathrm{C}_{2} \supset \ldots$

- $\mathrm{D}_{7} \supset \mathrm{A}_{6} \supset \mathrm{A}_{1} \oplus \mathrm{A}_{4} \supset \mathrm{A}_{1} \oplus \mathrm{A}_{1} \oplus \mathrm{A}_{2} \supset \ldots$ 
A primeira e a ultima cadeia podem ser eliminadas neste estágio pois apresentam 4 singletos, já a segunda é sobrevivente, então precisamos dar mais um passo na quebra:

- $D_{7} \supset A_{6} \supset A_{1} \oplus A_{4} \supset A_{1} \oplus C_{2} \supset A_{1} \oplus A_{1}$

- $D_{7} \supset A_{6} \supset A_{1} \oplus A_{4} \supset A_{1} \oplus C_{2} \supset A_{1} \oplus A_{1} \oplus A_{1}$

Após essa quebra aparecem na primeira cadeia 8 subespaços de dimensão impar e na segunda aparecem 4 singletos, logo podemos descartá-las.

\section{Sub-cadeias 5d:}

- $D_{7} \supset A_{6} \supset A_{2} \oplus A_{3} \supset A_{2} \oplus A_{2} \supset \ldots$

- $D_{7} \supset A_{6} \supset A_{2} \oplus A_{3} \supset A_{2} \oplus C_{2} \supset \ldots$

- $D_{7} \supset A_{6} \supset A_{2} \oplus A_{3} \supset A_{2} \oplus A_{1} \oplus A_{1} \supset \ldots$

Analisando as cadeias acima verificamos que as três apresentam excesso de subespaços de dimensão impar e, além disso, as duas primeiras apresentam excesso de singletos, portanto as três são eliminadas.

Com isso terminamos a análise nas sub-cadeias da cadeia 5 e verificamos que não existe nenhuma cadeia sobrevivente. Faremos agora a análise da cadeia 6, para isso listaremos todas as suas sub-cadeias:

Sub-cadeias 6a: $D_{7} \supset A_{1} \oplus A_{1} \oplus D_{5} \supset A_{1} \oplus A_{1} \oplus A_{4} \supset \ldots$

Sub-cadeias 6b: $D_{7} \supset A_{1} \oplus A_{1} \oplus D_{5} \supset A_{1} \oplus A_{1} \oplus B_{4} \supset \ldots$

Sub-cadeias 6c: $D_{7} \supset A_{1} \oplus A_{1} \oplus D_{5} \supset A_{1} \oplus A_{1} \oplus C_{2} \supset \ldots$

Sub-cadeias 6d: $D_{7} \supset A_{1} \oplus A_{1} \oplus D_{5} \supset A_{1} \oplus A_{1} \oplus A_{1} \oplus A_{1} \oplus A_{3} \supset \ldots$

Sub-cadeias 6e: $D_{7} \supset A_{1} \oplus A_{1} \oplus D_{5} \supset A_{1} \oplus A_{1} \oplus A_{1} \oplus B_{3} \supset \ldots$

Sub-cadeias 6f: $D_{7} \supset A_{1} \oplus A_{1} \oplus D_{5} \supset A_{1} \oplus A_{1} \oplus C_{2} \oplus C_{2} \supset \ldots$ 
Neste estágio de quebra todas as sub-cadeias acima são sobreviventes, então devemos prosseguir as quebras.

\section{Sub-cadeias 6a:}

- $\mathrm{D}_{7} \supset \mathrm{A}_{1} \oplus \mathrm{A}_{1} \oplus \mathrm{D}_{5} \supset \mathrm{A}_{1} \oplus \mathrm{A}_{1} \oplus \mathrm{A}_{4} \supset \mathrm{A}_{1} \oplus \mathrm{A}_{1} \oplus \mathrm{A}_{3} \supset A_{1} \oplus A_{1} \oplus A_{2} \supset \ldots$

- $\mathrm{D}_{7} \supset \mathrm{A}_{1} \oplus \mathrm{A}_{1} \oplus \mathrm{D}_{5} \supset \mathrm{A}_{1} \oplus \mathrm{A}_{1} \oplus \mathrm{A}_{4} \supset \mathrm{A}_{1} \oplus \mathrm{A}_{1} \oplus \mathrm{A}_{3} \supset \mathrm{A}_{1} \oplus \mathrm{A}_{1} \oplus \mathrm{C}_{2}$

$$
\supset A_{1} \oplus A_{1} \oplus A_{1} \oplus A_{1}
$$

- $\mathrm{D}_{7} \supset \mathrm{A}_{1} \oplus \mathrm{A}_{1} \oplus \mathrm{D}_{5} \supset \mathrm{A}_{1} \oplus \mathrm{A}_{1} \oplus \mathrm{A}_{4} \supset \mathrm{A}_{1} \oplus \mathrm{A}_{1} \oplus \mathrm{A}_{3} \supset \mathrm{A}_{1} \oplus \mathrm{A}_{1} \oplus \mathrm{C}_{2}$

$$
\supset A_{1} \oplus A_{1} \oplus A_{1}
$$

- $\mathrm{D}_{7} \supset \mathrm{A}_{1} \oplus \mathrm{A}_{1} \oplus \mathrm{D}_{5} \supset \mathrm{A}_{1} \oplus \mathrm{A}_{1} \oplus \mathrm{A}_{4} \supset \mathrm{A}_{1} \oplus \mathrm{A}_{1} \oplus \mathrm{A}_{3} \supset \mathrm{A}_{1} \oplus \mathrm{A}_{1} \oplus \mathrm{A}_{1} \oplus A_{1}$

- $\mathrm{D}_{7} \supset \mathrm{A}_{1} \oplus \mathrm{A}_{1} \oplus \mathrm{D}_{5} \supset \mathrm{A}_{1} \oplus \mathrm{A}_{1} \oplus \mathrm{A}_{4} \supset \mathrm{A}_{1} \oplus \mathrm{A}_{1} \oplus \mathrm{C}_{2} \supset \mathrm{A}_{1} \oplus \mathrm{A}_{1} \oplus \mathrm{A}_{1}$

- $\mathrm{D}_{7} \supset \mathbf{A}_{1} \oplus \mathbf{A}_{1} \oplus \mathrm{D}_{5} \supset \mathbf{A}_{1} \oplus \mathbf{A}_{1} \oplus \mathbf{A}_{4} \supset \mathbf{A}_{1} \oplus \mathbf{A}_{1} \oplus \mathbf{C}_{2} \supset \mathbf{A}_{1} \oplus \mathbf{A}_{1} \oplus \mathbf{A}_{1} \oplus \mathbf{A}_{1}$

- $\mathbf{D}_{7} \supset \mathbf{A}_{1} \oplus \mathbf{A}_{1} \oplus \mathbf{D}_{5} \supset \mathbf{A}_{1} \oplus \mathbf{A}_{1} \oplus \mathbf{A}_{\mathbf{4}} \supset \mathbf{A}_{1} \oplus \mathbf{A}_{1} \oplus \mathbf{A}_{1} \oplus \mathbf{A}_{2} \supset \mathbf{A}_{1} \oplus \mathbf{A}_{1} \oplus \mathbf{A}_{1} \oplus \mathbf{A}_{1}$

- $\mathrm{D}_{7} \supset \mathrm{A}_{1} \oplus \mathrm{A}_{1} \oplus \mathrm{D}_{5} \supset \mathrm{A}_{1} \oplus \mathrm{A}_{1} \oplus \mathrm{A}_{4} \supset \mathrm{A}_{1} \oplus \mathrm{A}_{1} \oplus \mathrm{A}_{1} \oplus \mathrm{A}_{2} \supset \mathrm{A}_{1} \oplus \mathrm{A}_{1} \oplus \mathrm{A}_{1} \oplus \mathrm{A}_{1}^{*}$

A primeira e a quarta dessas sub-cadeias são não-sobreviventes pois neste estágio da quebra as representações aparecem emparelhadas. A segunda, a terceira, a quinta e a oitava também são não-sobreviventes, pois em nenhuma delas conseguimos obter os sextupletos e tripletos. A sexta e a sétima são sobreviventes e devem ser submetidas à fase 2 .

\section{Sub-cadeias 6b:}

- $D_{7} \supset A_{1} \oplus A_{1} \oplus D_{5} \supset A_{1} \oplus A_{1} \oplus B_{4} \supset A_{1} \oplus A_{1} \oplus D_{4} \supset A_{1} \oplus A_{1} \oplus B_{3} \supset \ldots$

- $\mathrm{D}_{7} \supset \mathrm{A}_{1} \oplus \mathrm{A}_{1} \oplus \mathrm{D}_{5} \supset \mathrm{A}_{1} \oplus \mathrm{A}_{1} \oplus \mathrm{B}_{4} \supset \mathrm{A}_{1} \oplus \mathrm{A}_{1} \oplus \mathrm{D}_{4} \supset \mathrm{A}_{1} \oplus \mathrm{A}_{1} \oplus \mathrm{A}_{2} \supset \ldots$

- $\mathrm{D}_{7} \supset \mathrm{A}_{1} \oplus \mathrm{A}_{1} \oplus \mathrm{D}_{5} \supset \mathrm{A}_{1} \oplus \mathrm{A}_{1} \oplus \mathrm{B}_{4} \supset \mathrm{A}_{1} \oplus \mathrm{A}_{1} \oplus \mathrm{D}_{4} \supset \mathrm{A}_{1} \oplus \mathrm{A}_{1} \oplus \mathrm{A}_{1} \oplus \mathrm{C}_{2} \supset \ldots$

- $\mathrm{D}_{7} \supset \mathrm{A}_{1} \oplus \mathrm{A}_{1} \oplus \mathrm{D}_{5} \supset \mathrm{A}_{1} \oplus \dot{A}_{1} \oplus \mathrm{B}_{4} \supset \mathrm{A}_{1} \oplus \mathrm{A}_{1} \oplus \mathrm{D}_{4}$

$$
\supset \mathrm{A}_{1} \oplus \mathrm{A}_{1} \oplus \mathrm{A}_{1} \oplus \mathrm{A}_{1} \oplus \mathrm{A}_{1} \oplus \mathrm{A}_{1}
$$


- $\mathrm{D}_{7} \supset \mathrm{A}_{1} \oplus \mathrm{A}_{1} \oplus \mathrm{D}_{5} \supset \mathrm{A}_{1} \oplus \mathrm{A}_{1} \oplus \mathrm{B}_{4} \supset \mathrm{A}_{1} \oplus \mathrm{A}_{1} \oplus \mathrm{A}_{1} \oplus \mathrm{A}_{3} \supset \ldots$

- $\mathrm{D}_{7} \supset \mathrm{A}_{1} \oplus \mathrm{A}_{1} \oplus \mathrm{D}_{5} \supset \mathrm{A}_{1} \oplus \mathrm{A}_{1} \oplus \mathrm{B}_{4} \supset \mathrm{A}_{1} \oplus \mathrm{A}_{1} \oplus \mathrm{A}_{1} \oplus \mathrm{A}_{1} \oplus \mathrm{C}_{2} \supset \ldots$

- $\mathrm{D}_{7} \supset \mathrm{A}_{1} \oplus \mathrm{A}_{1} \oplus \mathrm{D}_{5} \supset \mathrm{A}_{1} \oplus \mathrm{A}_{1} \oplus \mathrm{B}_{4} \supset \mathrm{A}_{1} \oplus \mathrm{A}_{1} \oplus \mathrm{A}_{1} \oplus \mathrm{A}_{1}$

- $\mathrm{D}_{7} \supset \mathrm{A}_{1} \oplus \mathrm{A}_{1} \oplus \mathrm{D}_{5} \supset \mathrm{A}_{1} \oplus \mathrm{A}_{1} \oplus \mathrm{B}_{4} \supset \mathrm{A}_{1} \oplus \mathrm{A}_{1} \oplus \mathrm{A}_{1}$

Esta família de sub-cadeias é toda eliminada: a primeira, a segunda e a quinta são eliminadas por emparelhamento e as demais por não apresentarem representações capazes de reproduzirem os sextupletos e tripletos exigidos.

\section{Sub-cadeias 6c:}

- $\mathrm{D}_{7} \supset \mathrm{A}_{1} \oplus \mathrm{A}_{1} \oplus \mathrm{D}_{5} \supset \mathrm{A}_{1} \oplus \mathrm{A}_{1} \oplus \mathrm{C}_{2} \supset \mathrm{A}_{1} \oplus \mathrm{A}_{1} \oplus \mathrm{A}_{1}$

- $\mathbf{D}_{7} \supset \mathbf{A}_{1} \oplus \mathbf{A}_{1} \oplus \mathbf{D}_{5} \supset \mathbf{A}_{1} \oplus \mathbf{A}_{1} \oplus \mathbf{C}_{2} \supset \mathbf{A}_{1} \oplus \mathbf{A}_{1} \oplus \mathbf{A}_{1} \oplus \mathbf{A}_{1}$

A primeira cadeia é eliminada pois não é possível obter os tripletos e sextupletos necessários e a segunda é sobrevivente.

\section{Sub-cadeias 6d:}

- $\mathrm{D}_{7} \supset \mathrm{A}_{1} \oplus \mathrm{A}_{1} \oplus \mathrm{D}_{5} \supset \mathrm{A}_{1} \oplus \mathrm{A}_{1} \oplus \mathrm{A}_{1} \oplus \mathrm{A}_{1} \oplus \mathrm{A}_{3} \supset \mathrm{A}_{1} \oplus \mathrm{A}_{1} \oplus \mathrm{A}_{1} \oplus \mathrm{A}_{1} \oplus \mathrm{A}_{2} \supset \ldots$

- $\mathrm{D}_{7} \supset \mathrm{A}_{1} \oplus \mathrm{A}_{1} \oplus \mathrm{D}_{5} \supset \mathrm{A}_{1} \oplus \mathrm{A}_{1} \oplus \mathrm{A}_{1} \oplus \mathrm{A}_{1} \oplus \mathrm{A}_{3} \supset \mathrm{A}_{1} \oplus \mathrm{A}_{1} \oplus \mathrm{A}_{1} \oplus \mathrm{A}_{1} \oplus \mathrm{C}_{2} \supset \ldots$

- $\mathrm{D}_{7} \supset \mathrm{A}_{1} \oplus \mathrm{A}_{1} \oplus \mathrm{D}_{5} \supset \mathrm{A}_{1} \oplus \mathrm{A}_{1} \oplus \mathrm{A}_{1} \oplus \mathrm{A}_{1} \oplus \mathrm{A}_{3} \supset \mathrm{A}_{1} \oplus \mathrm{A}_{1} \oplus \mathrm{A}_{1} \oplus \mathrm{A}_{1} \oplus \mathrm{A}_{1} \oplus \mathrm{A}_{1}$

Aqui podemos eliminar todas as sub-cadeias pois a segunda é sobrevivente mas produz um resultado semelhante à cadeia acima em negrito, então podemos descartá-la, e as outras duas não produzem os tripletos e sextupletos necessários.

\section{Sub-cadeias 6e:}

- $\mathrm{D}_{7} \supset \mathrm{A}_{1} \oplus \mathrm{A}_{1} \oplus \mathrm{D}_{5} \supset \mathrm{A}_{1} \oplus \mathrm{A}_{1} \oplus \mathrm{A}_{1} \oplus \mathrm{B}_{3} \supset \mathrm{A}_{1} \oplus \mathrm{A}_{1} \oplus \mathrm{A}_{1} \oplus \mathrm{G}_{2} \supset \ldots$ 
- $\mathrm{D}_{7} \supset \mathrm{A}_{1} \oplus \mathrm{A}_{1} \oplus \mathrm{D}_{5} \supset \mathrm{A}_{1} \oplus \mathrm{A}_{1} \oplus \mathrm{A}_{1} \oplus \mathrm{B}_{3} \supset \mathrm{A}_{1} \oplus \mathrm{A}_{1} \oplus \mathrm{A}_{1} \oplus \mathrm{A}_{3} \supset \ldots$

- $\mathrm{D}_{7} \supset \mathrm{A}_{1} \oplus \mathrm{A}_{1} \oplus \mathrm{D}_{5} \supset \mathrm{A}_{1} \oplus \mathrm{A}_{1} \oplus \mathrm{A}_{1} \oplus \mathrm{B}_{3} \supset \mathrm{A}_{1} \oplus \mathrm{A}_{1} \oplus \mathrm{A}_{1} \oplus \mathrm{A}_{1} \oplus \mathrm{A}_{1} \oplus A_{1}$

A primeira cadeia é sobrevivente, então devemos prosseguir a quebra:

- $\mathrm{D}_{7} \supset \mathrm{A}_{1} \oplus \mathrm{A}_{1} \oplus \mathrm{D}_{5} \supset \mathrm{A}_{1} \oplus \mathrm{A}_{1} \oplus \mathrm{A}_{1} \oplus \mathrm{B}_{3} \supset \mathrm{A}_{1} \oplus \mathrm{A}_{1} \oplus \mathrm{A}_{1} \oplus \mathrm{G}_{2}$ $\supset A_{1} \oplus A_{1} \oplus A_{1} \oplus A_{2} \supset A_{1} \oplus A_{1} \oplus A_{1} \oplus A_{1}$

- $\mathrm{D}_{7} \supset \mathrm{A}_{1} \oplus \mathrm{A}_{1} \oplus \mathrm{D}_{5} \supset \mathrm{A}_{1} \oplus \mathrm{A}_{1} \oplus \mathrm{A}_{1} \oplus \mathrm{B}_{3} \supset \mathrm{A}_{1} \oplus \mathrm{A}_{1} \oplus \mathrm{A}_{1} \oplus \mathrm{G}_{2}$ $\supset \mathrm{A}_{1} \oplus \mathrm{A}_{1} \oplus \mathrm{A}_{1} \oplus \mathrm{A}_{1}$

- $\mathrm{D}_{7} \supset \mathrm{A}_{1} \oplus \mathrm{A}_{1} \oplus \mathrm{D}_{5} \supset \mathrm{A}_{1} \oplus \mathrm{A}_{1} \oplus \mathrm{A}_{1} \oplus \mathrm{B}_{3} \supset \mathrm{A}_{1} \oplus \mathrm{A}_{1} \oplus \mathrm{A}_{1} \oplus \mathrm{G}_{2}$ $\supset A_{1} \oplus A_{1} \oplus A_{1} \oplus A_{1} \oplus A_{1}$

Verificamos que estas cadeias são não-sobreviventes, pois elas não produzem os tripletos e sextupletos necessários. Prosseguindo, a segunda sub-cadeia também é sobrevivente e devemos quebra-la até a soma de $A_{1}$ 's:

- $\mathrm{D}_{7} \supset \mathrm{A}_{1} \oplus \mathrm{A}_{1} \oplus \mathrm{D}_{5} \supset \mathrm{A}_{1} \oplus \mathrm{A}_{1} \oplus \mathrm{A}_{1} \oplus \mathrm{B}_{3} \supset \mathrm{A}_{1} \oplus \mathrm{A}_{1} \oplus \mathrm{A}_{1} \oplus \mathrm{A}_{3}$ $\supset A_{1} \oplus A_{1} \oplus A_{1} \oplus A_{2} \supset A_{1} \oplus A_{1} \oplus A_{1} \oplus A_{1}$

- $\mathrm{D}_{7} \supset \mathrm{A}_{1} \oplus \mathrm{A}_{1} \oplus \mathrm{D}_{5} \supset \mathrm{A}_{1} \oplus \mathrm{A}_{1} \oplus \mathrm{A}_{1} \oplus \mathrm{B}_{3} \supset \mathrm{A}_{1} \oplus \mathrm{A}_{1} \oplus \mathrm{A}_{1} \oplus \mathrm{A}_{3}$ $\supset A_{1} \oplus A_{1} \oplus A_{1} \oplus C_{2} \supset A_{1} \oplus A_{1} \oplus A_{1} \oplus A_{1}$

- $\mathrm{D}_{7} \supset \mathrm{A}_{1} \oplus \mathrm{A}_{1} \oplus \mathrm{D}_{5} \supset \mathrm{A}_{1} \oplus \mathrm{A}_{1} \oplus \mathrm{A}_{1} \oplus \mathrm{B}_{3} \supset \mathrm{A}_{1} \oplus \mathrm{A}_{1} \oplus \mathrm{A}_{1} \oplus \mathrm{A}_{3}$ $\supset A_{1} \oplus A_{1} \oplus A_{1} \oplus C_{2} \supset A_{1} \oplus A_{1} \oplus A_{1} \oplus A_{1} \oplus A_{1}$

- $\mathrm{D}_{7} \supset \mathrm{A}_{1} \oplus \mathrm{A}_{1} \oplus \mathrm{D}_{5} \supset \mathrm{A}_{1} \oplus \mathrm{A}_{1} \oplus \mathrm{A}_{1} \oplus \mathrm{B}_{3} \supset \mathrm{A}_{1} \oplus \mathrm{A}_{1} \oplus \mathrm{A}_{1} \oplus \mathrm{A}_{3}$ $\supset A_{1} \oplus A_{l} \oplus A_{1} \oplus A_{1} \oplus A_{l}$

Verificamos que essas cadeias são não-sobreviventes, pois elas não produzem os tripletos e sextupletos necessários. Por fim, vetificamos que a ultima sub-cadeia das sub-cadeias 6e também é eliminada por esse mesmo motivo. 


\section{Sub-cadeias 6f:}

- $\mathrm{D}_{7} \supset \mathrm{A}_{1} \oplus \mathrm{A}_{1} \oplus \mathrm{D}_{5} \supset \mathrm{A}_{1} \oplus \mathrm{A}_{1} \oplus \mathrm{C}_{2} \oplus \mathrm{C}_{2} \supset \mathrm{A}_{1} \oplus \mathrm{A}_{1} \oplus \mathrm{A}_{1} \oplus \mathrm{A}_{1}$

- $\mathrm{D}_{7} \supset \mathrm{A}_{1} \oplus \mathrm{A}_{1} \oplus \mathrm{D}_{5} \supset \mathrm{A}_{1} \oplus \mathrm{A}_{1} \oplus \mathrm{C}_{2} \oplus \mathrm{C}_{2} \supset \mathrm{A}_{1} \oplus \mathrm{A}_{1} \oplus \mathrm{A}_{1} \oplus \mathrm{A}_{1} \oplus \mathrm{A}_{1}$

- $\mathrm{D}_{7} \supset \mathrm{A}_{1} \oplus \mathrm{A}_{1} \oplus \mathrm{D}_{5} \supset \mathrm{A}_{1} \oplus \mathrm{A}_{1} \oplus \mathrm{C}_{2} \oplus \mathrm{C}_{2} \supset \mathrm{A}_{1} \oplus \mathrm{A}_{1} \oplus \mathrm{A}_{1} \oplus \mathrm{A}_{1} \oplus \mathrm{A}_{1} \oplus \mathrm{A}_{1}$

Verificamos que essas cadeias são não-sobreviventes, pois elas não produzem os tripletos e sextupletos necessários. Com isso finalizamos a análise da cadeia 6 , da qual obtivemos 3 cadeias sobreviventes, as quais serão analisadas na fase 2 na próxima seção.

Analisaremos agora a cadeia 7 e iniciaremos listando suas sub-cadeias:

Sub-cadeias 7a: $D_{7} \supset A_{3} \oplus D_{4} \supset A_{3} \oplus B_{3}$

Sub-cadeias 7b: $D_{7} \supset A_{3} \oplus D_{4} \supset A_{3} \oplus A_{2}$

Sub-cadeias 7c: $D_{7} \supset A_{3} \oplus D_{4} \supset A_{3} \oplus A_{1} \oplus C_{2}$

Sub-cadeias 7d: $D_{7} \supset A_{3} \oplus D_{4} \supset A_{3} \oplus A_{1} \oplus A_{1} \oplus A_{1} \oplus A_{1}$

Analisando este primeiro estágio da quebra de simetria da cadeia 7, podemos eliminar as subcadeias $7 \mathrm{a}$ e $7 \mathrm{~b}$ pois como as representações no $\mathrm{A}_{3}$ são conjugadas então todas essas cadeias são eliminadas por emparelhamento. As sub-cadeias $7 \mathrm{c}$ e $7 \mathrm{~d}$ são sobreviventes, então devemos prosseguir as quebras:

\section{Sub-cadeias 7c:}

- $\mathbf{D}_{7} \supset \mathbf{A}_{3} \oplus \mathbf{D}_{4} \supset \mathbf{A}_{3} \oplus \mathbf{A}_{1} \oplus \mathbf{C}_{2} \supset \mathbf{A}_{3} \oplus \mathbf{A}_{1} \oplus \mathbf{A}_{1} \supset \mathbf{A}_{2} \oplus \mathbf{A}_{1} \oplus \mathbf{A}_{1} \supset \mathbf{A}_{1} \oplus \mathbf{A}_{1} \oplus \mathbf{A}_{1}$

- $\mathrm{D}_{7} \supset \mathrm{A}_{3} \oplus \mathrm{D}_{4} \supset \mathrm{A}_{3} \oplus \mathrm{A}_{1} \oplus \mathrm{C}_{2} \supset \mathrm{A}_{3} \oplus \mathrm{A}_{1} \oplus \mathrm{A}_{1} \supset \mathrm{A}_{2} \oplus \mathrm{A}_{1} \oplus \mathrm{A}_{1} \supset \mathrm{A}_{1}^{*} \oplus \mathrm{A}_{1} \oplus \mathrm{A}_{1}$

- $\mathrm{D}_{7} \supset \mathrm{A}_{3} \oplus \mathrm{D}_{4} \supset \mathrm{A}_{3} \oplus \mathrm{A}_{1} \oplus \mathrm{C}_{2} \supset \mathrm{A}_{3} \oplus \mathrm{A}_{1} \oplus \mathrm{A}_{1} \supset \mathrm{C}_{2} \oplus \mathrm{A}_{1} \oplus \mathrm{A}_{1} \supset \mathrm{A}_{1} \oplus \mathrm{A}_{1} \oplus \mathrm{A}_{1}$

- $\mathrm{D}_{7} \supset \mathrm{A}_{3} \oplus \mathrm{D}_{4} \supset \mathrm{A}_{3} \oplus \mathrm{A}_{1} \oplus \mathrm{C}_{2} \supset \mathrm{A}_{3} \oplus \mathrm{A}_{1} \oplus \mathrm{A}_{1} \supset \mathrm{C}_{2} \oplus \mathrm{A}_{1} \oplus \mathrm{A}_{1}$ $\supset A_{1} \oplus A_{1} \oplus A_{1} \oplus A_{1}$ 
- $\mathrm{D}_{7} \supset \mathrm{A}_{3} \oplus \mathrm{D}_{4} \supset \mathrm{A}_{3} \oplus \mathrm{A}_{1} \oplus \mathrm{C}_{2} \supset \mathrm{A}_{3} \oplus \mathrm{A}_{1} \oplus \mathrm{A}_{1} \supset \mathrm{A}_{1} \oplus \mathrm{A}_{1} \oplus \mathrm{A}_{1} \oplus \mathrm{A}_{1}$

- $\mathrm{D}_{7} \supset \mathbf{A}_{3} \oplus \mathbf{D}_{4} \supset \mathbf{A}_{3} \oplus \mathbf{A}_{1} \oplus \mathbf{C}_{2} \supset \mathbf{A}_{3} \oplus \mathbf{A}_{\mathbf{1}} \oplus \mathbf{A}_{1} \oplus \mathbf{A}_{1} \supset \mathbf{A}_{\mathbf{2}} \oplus \mathbf{A}_{\mathbf{1}} \oplus \mathbf{A}_{1} \oplus \mathbf{A}_{1}$ $\supset \mathbf{A}_{1} \oplus \mathbf{A}_{1} \oplus \mathbf{A}_{1} \oplus \mathbf{A}_{1}$

- $\mathrm{D}_{7} \supset \mathrm{A}_{3} \oplus \mathrm{D}_{4} \supset \mathrm{A}_{3} \oplus \mathrm{A}_{1} \oplus \mathrm{C}_{2} \supset \mathrm{A}_{3} \oplus \mathrm{A}_{1} \oplus \mathrm{A}_{1} \oplus \mathrm{A}_{1} \supset \mathrm{A}_{2} \oplus \mathrm{A}_{1} \oplus \mathrm{A}_{1} \oplus \mathrm{A}_{1}$ $\supset A_{1}^{*} \oplus A_{1} \oplus A_{1} \oplus A_{1}$

- $\mathrm{D}_{7} \supset \mathrm{A}_{3} \oplus \mathrm{D}_{4} \supset \mathrm{A}_{3} \oplus \mathrm{A}_{1} \oplus \mathrm{C}_{2} \supset \mathrm{A}_{3} \oplus \mathrm{A}_{1} \oplus \mathrm{A}_{1} \oplus \mathrm{A}_{1} \supset \mathrm{C}_{2} \oplus \mathrm{A}_{1} \oplus \mathrm{A}_{1} \oplus \mathrm{A}_{1}$ $\supset A_{1} \oplus A_{1} \oplus A_{1} \oplus A_{1}$

- $\mathrm{D}_{7} \supset \mathrm{A}_{3} \oplus \mathrm{D}_{4} \supset \mathrm{A}_{3} \oplus \mathrm{A}_{1} \oplus \mathrm{C}_{2} \supset \mathrm{A}_{3} \oplus \mathrm{A}_{1} \oplus \mathrm{A}_{1} \oplus \mathrm{A}_{1} \supset \mathrm{C}_{2} \oplus \mathrm{A}_{1} \oplus \mathrm{A}_{1} \oplus \mathrm{A}_{1}$ $\supset A_{1} \oplus A_{1} \oplus A_{1} \oplus A_{1} \oplus A_{l}$

- $D_{7} \supset A_{3} \oplus D_{4} \supset A_{3} \oplus A_{1} \oplus C_{2} \supset A_{3} \oplus A_{1} \oplus A_{1} \oplus A_{1} \supset A_{1} \oplus A_{1} \oplus A_{1} \oplus A_{1} \oplus A_{1}$

Analisando as cadeias acima, temos que a primeira e a sexta cadeia são sobreviventes, mas as demais são eliminadas, pois elas não apresentam subespaços capazes de gerar os tripletos e sextupletos necessários.

\section{Sub-cadeias 7d:}

- $\mathrm{D}_{7} \supset \mathrm{A}_{3} \oplus \mathrm{D}_{4} \supset \mathrm{A}_{3} \oplus \mathbf{A}_{1} \oplus \mathbf{A}_{1} \oplus \mathbf{A}_{1} \oplus \mathbf{A}_{1} \supset \mathbf{A}_{2} \oplus \mathbf{A}_{1} \oplus \mathbf{A}_{1} \oplus \mathbf{A}_{1} \oplus \mathbf{A}_{1}$ $\supset \mathbf{A}_{1} \oplus \mathbf{A}_{1} \oplus \mathbf{A}_{1} \oplus \mathbf{A}_{1} \oplus \mathbf{A}_{1}$

- $\mathrm{D}_{7} \supset \mathrm{A}_{3} \oplus \mathrm{D}_{4} \supset \mathrm{A}_{3} \oplus \mathrm{A}_{1} \oplus \mathrm{A}_{1} \oplus \mathrm{A}_{1} \oplus \mathrm{A}_{1} \supset \mathrm{A}_{2} \oplus \mathrm{A}_{1} \oplus \mathrm{A}_{1} \oplus \mathrm{A}_{1} \oplus \mathrm{A}_{1}$ $\supset A_{1}^{*} \oplus A_{1} \oplus A_{1} \oplus A_{1} \oplus A_{1}$

- $\mathrm{D}_{7} \supset \mathrm{A}_{3} \oplus \mathrm{D}_{4} \supset \mathrm{A}_{3} \oplus \mathrm{A}_{1} \oplus \mathrm{A}_{1} \oplus \mathrm{A}_{1} \oplus \mathrm{A}_{1} \supset \mathrm{C}_{2} \oplus \mathrm{A}_{1} \oplus \mathrm{A}_{1} \oplus \mathrm{A}_{1} \oplus \mathrm{A}_{1}$ $\supset A_{1} \oplus A_{1} \oplus A_{1} \oplus A_{1} \oplus A_{1}$

- $\mathrm{D}_{7} \supset \mathrm{A}_{3} \oplus \mathrm{D}_{4} \supset \mathrm{A}_{3} \oplus \mathrm{A}_{1} \oplus \mathrm{A}_{1} \oplus \mathrm{A}_{1} \oplus \mathrm{A}_{1} \supset \mathrm{A}_{2} \oplus \mathrm{A}_{1} \oplus \mathrm{A}_{1} \oplus \mathrm{A}_{1} \oplus \mathrm{A}_{1}$ $\supset A_{1} \oplus A_{1} \oplus A_{1} \oplus A_{1} \oplus A_{1} \oplus A_{1}$

- $D_{7} \supset A_{3} \oplus D_{4} \supset A_{3} \oplus A_{1} \oplus \cdot A_{1} \oplus A_{1} \oplus A_{1} \supset A_{1} \oplus A_{1} \oplus A_{1} \oplus A_{1} \oplus A_{1} \oplus A_{1}$ 
Analisando as cadeias acima temos que somente a primeira é sobrevivente; as demais são eliminadas por não apresentarem subespaços capazes de reproduzirem os tripletos e sextupletos necessários.

Faremos agora a análise da cadeia 8 , a qual possui as seguintes sub-cadeias:

Sub-cadeias 8a: $D_{7} \supset A_{1} \oplus B_{5} \supset A_{1} \oplus D_{5} \supset \ldots$

Sub-cadeia 8b: $D_{7} \supset A_{1} \oplus B_{5} \supset A_{1} \oplus A_{1}$

Sub-cadeias 8c: $D_{7} \supset A_{1} \oplus B_{5} \supset A_{1} \oplus A_{1} \oplus A_{1} \oplus B_{3} \supset \ldots$

Sub-cadeias 8d: $D_{7} \supset A_{1} \oplus B_{5} \supset A_{1} \oplus A_{1} \oplus D_{4} \supset \ldots$

Sub-cadeias 8e: $\mathrm{D}_{7} \supset \mathrm{A}_{1} \oplus \mathrm{B}_{5} \supset \mathrm{A}_{1} \oplus \mathrm{A}_{3} \oplus \mathrm{C}_{2} \supset \ldots$

Analisando as sub-cadeias acima, observamos que $8 \mathrm{a}, 8 \mathrm{~b}$ e $8 \mathrm{e}$ já são eliminadas neste estágio da quebra, pois as representações das sub-cadeias $8 \mathrm{a}$ e $8 \mathrm{e}$ aparecem emparelhadas e a sub-cadeia 8b chegou a soma de $A_{1}$ 's e não apresenta subespaços capazes de gerar os sextupletos e os tripletos. As sub-cadeias $8 \mathrm{c}$ e $8 \mathrm{~d}$ são sobreviventes e devem continuar quebrando:

\section{Sub-cadeias 8c:}

- $D_{7} \supset A_{1} \oplus B_{5} \supset A_{1} \oplus A_{1} \oplus A_{1} \oplus B_{3} \supset A_{1} \oplus A_{1} \oplus A_{1} \oplus G_{2} \supset \ldots$

- $\mathrm{D}_{7} \supset \mathrm{A}_{1} \oplus \mathrm{B}_{5} \supset \mathrm{A}_{1} \oplus \mathrm{A}_{1} \oplus \mathrm{A}_{1} \oplus \mathrm{B}_{3} \supset \mathrm{A}_{1} \oplus \mathrm{A}_{1} \oplus \mathrm{A}_{1} \oplus \mathrm{A}_{3} \supset \ldots$

- $\mathrm{D}_{7} \supset \mathrm{A}_{1} \oplus \mathrm{B}_{5} \supset \mathrm{A}_{1} \oplus \mathrm{A}_{1} \oplus \mathrm{A}_{1} \oplus \mathrm{B}_{3} \supset \mathrm{A}_{1} \oplus \mathrm{A}_{1} \oplus \mathrm{A}_{1} \oplus \mathrm{A}_{1} \oplus \mathrm{A}_{1} \oplus \mathrm{A}_{1}$

Analisando as cadeias acima podemos eliminar a segunda, devido ao emparelhamento, e a terceira por não gerar os tripletos e sextupletos, enquanto que a primeira é sobrevivente $\mathrm{e}$ devemos prosseguir a quebra:

- $\mathrm{D}_{7} \supset \mathrm{A}_{1} \oplus \mathrm{B}_{5} \supset \mathrm{A}_{1} \oplus \mathrm{A}_{1} \oplus \mathrm{A}_{1} \oplus \mathrm{B}_{3} \supset \mathrm{A}_{1} \oplus \mathrm{A}_{1} \oplus \mathrm{A}_{1} \oplus \mathrm{G}_{2} \supset \mathrm{A}_{1} \oplus \mathrm{A}_{1} \oplus \mathrm{A}_{1} \oplus \mathrm{A}_{2}$

- $\mathrm{D}_{7} \supset \mathrm{A}_{1} \oplus \mathrm{B}_{5} \supset \mathrm{A}_{1} \oplus \mathrm{A}_{1} \oplus \mathrm{A}_{1} \oplus \mathrm{B}_{3} \supset \mathrm{A}_{1} \oplus \mathrm{A}_{1} \oplus \mathrm{A}_{1} \oplus \mathrm{G}_{2} \supset \mathrm{A}_{1} \oplus \mathrm{A}_{1} \oplus \mathrm{A}_{1} \oplus A_{1}$ 
- $\mathrm{D}_{7} \supset \mathrm{A}_{1} \oplus \mathrm{B}_{5} \supset \mathrm{A}_{1} \oplus \mathrm{A}_{1} \oplus \mathrm{A}_{1} \oplus \mathrm{B}_{3} \supset \mathrm{A}_{1} \oplus \mathrm{A}_{1} \oplus \mathrm{A}_{1} \oplus \mathrm{G}_{2}$

$\supset A_{1} \oplus A_{1} \oplus A_{1} \oplus A_{1} \oplus A_{1}$

Observamos que na primeira cadeia as representaçōes de $A_{2}$ são conjugadas, então ela é eliminada devido ao emparelhamento. A segunda é eliminada por não gerar os tripletos e sextupletos, e a ultima é sobrevivente, mas reproduz resultados semelhantes aos da cadeia 10 , portanto ela pode ser eliminada.

\section{Sub-cadeias 8d:}

- $\mathrm{D}_{7} \supset \mathrm{A}_{1} \oplus \mathrm{B}_{5} \supset \mathrm{A}_{1} \oplus \mathrm{A}_{1} \oplus \mathrm{D}_{4} \supset \mathrm{A}_{1} \oplus \mathrm{A}_{1} \oplus \mathrm{B}_{3} \supset \ldots$

- $\mathrm{D}_{7} \supset \mathrm{A}_{1} \oplus \mathrm{B}_{5} \supset \mathrm{A}_{1} \oplus \mathrm{A}_{1} \oplus \mathrm{D}_{4} \supset \mathrm{A}_{1} \oplus \mathrm{A}_{1} \oplus \mathrm{A}_{2} \supset \ldots$

- $\mathrm{D}_{7} \supset \mathrm{A}_{1} \oplus \mathrm{B}_{5} \supset \mathrm{A}_{1} \oplus \mathrm{A}_{1} \oplus \mathrm{D}_{4} \supset \mathrm{A}_{1} \oplus \mathrm{A}_{1} \oplus \mathrm{A}_{1} \oplus \mathrm{C}_{2} \supset \mathrm{A}_{1} \oplus \mathrm{A}_{1} \oplus \mathrm{A}_{1} \oplus \mathrm{A}_{1}$

- $\mathrm{D}_{7} \supset \mathrm{A}_{1} \oplus \mathrm{B}_{5} \supset \mathrm{A}_{1} \oplus \mathrm{A}_{1} \oplus \mathrm{D}_{4} \supset \mathrm{A}_{1} \oplus \mathrm{A}_{1} \oplus \mathrm{A}_{1} \oplus \mathrm{C}_{2} \supset \mathrm{A}_{1} \oplus \mathrm{A}_{1} \oplus \mathrm{A}_{1} \oplus \mathrm{A}_{1} \oplus \mathrm{A}_{1}$

- $\mathrm{D}_{7} \supset \mathrm{A}_{1} \oplus \mathrm{B}_{5} \supset \mathrm{A}_{1} \oplus \mathrm{A}_{1} \oplus \mathrm{D}_{4} \supset \mathrm{A}_{1} \oplus \mathrm{A}_{1} \oplus \mathrm{A}_{1} \oplus \mathrm{A}_{1} \oplus \mathrm{A}_{1} \oplus \mathrm{A}_{1}$

Todas essas cadeias são eliminadas: a primeira e a segunda por emparelhamento, e as três ultimas por não apresentarem subespaços capazes de reproduzirem os tripletos e sextupletos necessários.

Analisaremos agora a cadeia 9, cujas sub-cadeias são:

Sub-cadeias 9a: $D_{7} \supset C_{2} \oplus B_{4} \supset C_{2} \oplus D_{4} \supset \ldots$

Sub-cadeias $9 \mathrm{~b}: \mathrm{D}_{7} \supset \mathrm{C}_{2} \oplus \mathrm{B}_{4} \supset \mathrm{C}_{2} \oplus \mathrm{A}_{1} \supset \ldots$

Sub-cadeias 9c: $D_{7} \supset C_{2} \oplus B_{4} \supset C_{2} \oplus A_{1} \oplus A_{1} \supset \ldots$

Sub-cadeias 9d: $D_{7} \supset C_{2} \oplus B_{4} \supset C_{2} \oplus A_{1} \oplus A_{1} \oplus C_{2} \supset \ldots$

Sub-cadeias 9e: $\mathrm{D}_{7} \supset \mathrm{C}_{2} \oplus \mathrm{B}_{4} \supset \mathrm{C}_{2} \oplus \mathrm{A}_{1} \oplus \mathrm{A}_{3} \supset \ldots$

As sub-cadeias 9e são eliminadas pois no início do processo de quebra de simetria já apresentam emparelhamento, as demais são sobreviventes e devemos prosseguir as quebras: 
Sub-cadeias 9a:

- $\mathrm{D}_{7} \supset \mathrm{C}_{2} \oplus \mathrm{B}_{4} \supset \mathrm{C}_{2} \oplus \mathrm{D}_{4} \supset \mathrm{C}_{2} \oplus \mathrm{B}_{3} \supset \ldots$

- $\mathrm{D}_{7} \supset \mathrm{C}_{2} \oplus \mathrm{B}_{4} \supset \mathrm{C}_{2} \oplus \mathrm{D}_{4} \supset \mathrm{C}_{2} \oplus \mathrm{A}_{2} \supset \ldots$

- $\mathrm{D}_{7} \supset \mathrm{C}_{2} \oplus \mathrm{B}_{4} \supset \mathrm{C}_{2} \oplus \mathrm{D}_{4} \supset \mathrm{C}_{2} \oplus \mathrm{A}_{1} \oplus \mathrm{C}_{2} \supset \ldots$

- $\mathrm{D}_{7} \supset \mathrm{C}_{2} \oplus \mathrm{B}_{4} \supset \mathrm{C}_{2} \oplus \mathrm{D}_{4} \supset \mathrm{C}_{2} \oplus \mathrm{A}_{1} \oplus \mathrm{A}_{1} \oplus \mathrm{A}_{1} \oplus \mathrm{A}_{1} \supset \ldots$

A primeira e a segunda cadeia apresentam emparelhamento, a terceira reproduz resultados da análise na cadeia 7 (sub-cadeia 7c - terceira cadeia, a qual é eliminada), e a última reproduz resultados da análise da cadeia 7 (sub-cadeia $7 \mathrm{~d}$ - terceira cadeia, que também é eliminada), logo todas são não-sobreviventes.

Sub-cadeias 9b:

- $\mathrm{D}_{7} \supset \mathrm{C}_{2} \oplus \mathrm{B}_{4} \supset \mathrm{C}_{2} \oplus \mathrm{A}_{1} \supset \mathrm{A}_{1} \oplus \mathrm{A}_{1}$

- $\mathrm{D}_{7} \supset \mathrm{C}_{2} \oplus \mathrm{B}_{4} \supset \mathrm{C}_{2} \oplus \mathrm{A}_{1} \supset \mathrm{A}_{1} \oplus \mathrm{A}_{1} \oplus \mathrm{A}_{1}$

As duas cadeias não apresentam representações capazes de gerar os tripletos e os sextupletos, logo são eliminadas.

Sub-cadeias 9c:

- $\mathrm{D}_{7} \supset \mathrm{C}_{2} \oplus \mathrm{B}_{4} \supset \mathrm{C}_{2} \oplus \mathrm{A}_{1} \oplus \mathrm{A}_{1} \supset \mathrm{A}_{1} \oplus \mathrm{A}_{1} \oplus \mathrm{A}_{1}$

- $\mathrm{D}_{7} \supset \mathrm{C}_{2} \oplus \mathrm{B}_{4} \supset \mathrm{C}_{2} \oplus \mathrm{A}_{1} \oplus \mathrm{A}_{1} \supset \mathrm{A}_{1} \oplus \mathrm{A}_{1} \oplus \mathrm{A}_{1} \oplus \mathrm{A}_{1}$

Essas cadeias também são eliminadas por não gerarem os tripletos e os sextupletos.

Sub-cadeias 9d:

- $\mathrm{D}_{7} \supset \mathrm{C}_{2} \oplus \mathrm{B}_{4} \supset \mathrm{C}_{2} \oplus \mathrm{A}_{1} \oplus \mathrm{A}_{1} \oplus \mathrm{C}_{2} \supset \mathrm{C}_{2} \oplus \mathrm{A}_{1} \oplus \mathrm{A}_{1} \oplus \mathrm{A}_{1} \supset \mathrm{A}_{1} \oplus \mathrm{A}_{1} \oplus \mathrm{A}_{1} \oplus \mathrm{A}_{1}$

- $\mathrm{D}_{7} \supset \mathrm{C}_{2} \oplus \mathrm{B}_{4} \supset \mathrm{C}_{2} \oplus \mathrm{A}_{1} \oplus \mathrm{A}_{1} \oplus \mathrm{C}_{2} \supset \mathrm{C}_{2} \oplus \mathrm{A}_{1} \oplus \mathrm{A}_{1} \oplus \mathrm{A}_{1} \oplus \mathrm{A}_{1}$ $\supset A_{1} \oplus A_{1} \oplus A_{1} \oplus A_{1} \oplus A_{1}$ 
- $\mathrm{D}_{7} \supset \mathrm{C}_{2} \oplus \mathrm{B}_{4} \supset \mathrm{C}_{2} \oplus \mathrm{A}_{1} \oplus \mathrm{A}_{1} \oplus \mathrm{C}_{2} \supset \mathrm{C}_{2} \oplus \mathrm{A}_{1} \oplus \mathrm{A}_{1} \oplus \mathrm{A}_{1} \oplus \mathrm{A}_{1}$

$\supset A_{1} \oplus A_{1} \oplus A_{1} \oplus A_{1} \oplus A_{1} \oplus A_{1}$

As cadeias acima não apresentam representações que possam gerar os tripletos e quadrupletos necessários, portanto elas são eliminadas.

Para finalizar a análise na fase 1 das cadeias candidatas a reproduzirem a degenerescência do código genético, faremos agora a análise da cadeia 10, a qual se divide nas seguintes sub-cadeias:

Sub-cadeias 10a: $\mathrm{D}_{7} \supset \mathrm{B}_{3} \oplus \mathrm{B}_{3} \supset \mathrm{B}_{3} \oplus \mathrm{A}_{3} \supset \ldots$

Sub-cadeias 10b: $D_{7} \supset B_{3} \oplus B_{3} \supset B_{3} \oplus G_{2} \supset \ldots$

Sub-cadeias 10c: $D_{7} \supset B_{3} \oplus B_{3} \supset B_{3} \oplus A_{1} \oplus A_{1} \oplus A_{1} \supset \ldots$

Nas sub-cadeias $10 \mathrm{a}$, as representações de $\mathrm{A}_{3}$ são conjugadas, então todas essas sub-cadeias apresentam-se emparelhadas, logo devem ser eliminadas, enquanto que nas outras duas temos que prosseguir a quebra:

\section{Sub-cadeias 10b:}

- $\mathrm{D}_{7} \supset \mathrm{B}_{3} \oplus \mathrm{B}_{3} \supset \mathrm{B}_{3} \oplus \mathrm{G}_{2} \supset \mathrm{A}_{3} \oplus \mathrm{G}_{2} \supset \ldots$

- $\mathrm{D}_{7} \supset \mathrm{B}_{3} \oplus \mathrm{B}_{3} \supset \mathrm{B}_{3} \oplus \mathrm{G}_{2} \supset \mathrm{G}_{2} \oplus \mathrm{G}_{2} \supset \ldots$

- $\mathrm{D}_{7} \supset \mathrm{B}_{3} \oplus \mathrm{B}_{3} \supset \mathrm{B}_{3} \oplus \mathrm{G}_{2} \supset \mathrm{A}_{1} \oplus \mathrm{A}_{1} \oplus \mathrm{A}_{1} \oplus \mathrm{G}_{2} \supset \ldots$

$\mathrm{Na}$ primeira cadeia as representações de $\mathrm{A}_{3}$ vem em pares conjugados, então elas são eliminadas por emparelhamento, enquanto que as duas ultimas são sobreviventes, então devemos continuar a quebra:

- $\mathrm{D}_{7} \supset \mathrm{B}_{3} \oplus \mathrm{B}_{3} \supset \mathrm{B}_{3} \oplus \mathrm{G}_{2} \supset \mathrm{G}_{2} \oplus \mathrm{G}_{2} \supset \mathrm{G}_{2} \oplus \mathrm{A}_{2} \supset \mathrm{G}_{2} \oplus \mathrm{A}_{1} \supset \mathrm{A}_{1} \oplus \mathrm{A}_{1}$

- $\mathrm{D}_{7} \supset \mathrm{B}_{3} \oplus \mathrm{B}_{3} \supset \mathrm{B}_{3} \oplus \mathrm{G}_{2} \supset \mathrm{G}_{2} \oplus \mathrm{G}_{2} \supset \mathrm{G}_{2} \oplus \mathrm{A}_{2} \supset \mathrm{G}_{2} \oplus \mathrm{A}_{1} \supset \mathrm{A}_{1} \oplus \mathrm{A}_{1} \oplus \mathrm{A}_{1}$

- $\mathrm{D}_{7} \supset \mathrm{B}_{3} \oplus \mathrm{B}_{3} \supset \mathrm{B}_{3} \oplus \mathrm{G}_{2} \supset \mathrm{G}_{2} \oplus \mathrm{G}_{2} \supset \mathrm{G}_{2} \oplus \mathrm{A}_{2} \supset \mathrm{G}_{2} \oplus \mathrm{A}_{1} \supset \mathrm{A}_{2} \oplus \mathrm{A}_{1} \supset \mathrm{A}_{1} \oplus \mathrm{A}_{1}$ 
- $\mathrm{D}_{7} \supset \mathrm{B}_{3} \oplus \mathrm{B}_{3} \supset \mathrm{B}_{3} \oplus \mathrm{G}_{2} \supset \mathrm{G}_{2} \oplus \mathrm{G}_{2} \supset \mathrm{G}_{2} \oplus \mathrm{A}_{2} \supset \mathrm{G}_{2} \oplus \mathrm{A}_{1} \supset \mathrm{A}_{2} \oplus \mathrm{A}_{1} \supset \mathrm{A}_{1}^{*} \oplus \mathrm{A}_{1}$

- $\mathrm{D}_{7} \supset \mathrm{B}_{3} \oplus \mathrm{B}_{3} \supset \mathrm{B}_{3} \oplus \mathrm{G}_{2} \supset \mathrm{G}_{2} \oplus \mathrm{G}_{2} \supset \mathrm{G}_{2} \oplus \mathrm{A}_{2} \supset \mathrm{G}_{2} \oplus \mathrm{A}_{1}^{*} \supset \mathrm{A}_{1} \oplus \mathrm{A}_{i}$

- $\mathrm{D}_{7} \supset \mathrm{B}_{3} \oplus \mathrm{B}_{3} \supset \mathrm{B}_{3} \oplus \mathrm{G}_{2} \supset \mathrm{G}_{2} \oplus \mathrm{G}_{2} \supset \mathrm{G}_{2} \oplus \mathrm{A}_{2} \supset \mathrm{G}_{2} \oplus \mathrm{A}_{1}^{*} \supset \mathrm{A}_{1} \oplus \mathrm{A}_{1} \oplus \mathrm{A}_{1}^{*}$

- $\mathrm{D}_{7} \supset \mathrm{B}_{3} \oplus \mathrm{B}_{3} \supset \mathrm{B}_{3} \oplus \mathrm{G}_{2} \supset \mathrm{G}_{2} \oplus \mathrm{G}_{2} \supset \mathrm{G}_{2} \oplus \mathrm{A}_{2} \supset \mathrm{G}_{2} \oplus \mathrm{A}_{1}^{*} \supset \mathrm{A}_{2} \oplus \mathrm{A}_{1}^{*} \supset \mathrm{A}_{1} \oplus \mathrm{A}_{1}^{*}$

- $\mathrm{D}_{7} \supset \mathrm{B}_{3} \oplus \mathrm{B}_{3} \supset \mathrm{B}_{3} \oplus \mathrm{G}_{2} \supset \mathrm{G}_{2} \oplus \mathrm{G}_{2} \supset \mathrm{G}_{2} \oplus \mathrm{A}_{2} \supset \mathrm{G}_{2} \oplus \mathrm{A}_{1}^{*} \supset \mathrm{A}_{2} \oplus \mathrm{A}_{1}^{*} \supset \mathrm{A}_{1}^{*} \oplus \mathrm{A}_{1}^{*}$

- $\mathrm{D}_{7} \supset \mathrm{B}_{3} \oplus \mathrm{B}_{3} \supset \mathrm{B}_{3} \oplus \mathrm{G}_{2} \supset \mathrm{G}_{2} \oplus \mathrm{G}_{2} \supset \mathrm{G}_{2} \oplus \mathrm{A}_{1} \supset \mathrm{A}_{1} \oplus \mathrm{A}_{1}$

- $\mathrm{D}_{7} \supset \mathrm{B}_{3} \oplus \mathrm{B}_{3} \supset \mathrm{B}_{3} \oplus \mathbf{G}_{2} \supset \mathbf{G}_{2} \oplus \mathbf{G}_{2} \supset \mathbf{G}_{2} \oplus \mathrm{A}_{1} \supset A_{1} \oplus A_{1} \oplus A_{1}$

- $\mathrm{D}_{7} \supset \mathrm{B}_{3} \oplus \mathrm{B}_{3} \supset \mathrm{B}_{3} \oplus \mathrm{G}_{2} \supset \mathrm{G}_{2} \oplus \mathrm{G}_{2} \supset \mathrm{G}_{2} \oplus \mathrm{A}_{1} \supset \mathrm{A}_{2} \oplus \mathrm{A}_{1} \supset \mathrm{A}_{1} \oplus \mathrm{A}_{1}$

- $\mathrm{D}_{7} \supset \mathrm{B}_{3} \oplus \mathrm{B}_{3} \supset \mathrm{B}_{3} \oplus \mathrm{G}_{2} \supset \mathrm{G}_{2} \oplus \mathrm{G}_{2} \supset \mathrm{G}_{2} \oplus \mathrm{A}_{1} \supset \mathrm{A}_{2} \oplus \mathrm{A}_{1} \supset \mathrm{A}_{1}^{*} \oplus \mathrm{A}_{1}$

- $\mathrm{D}_{7} \supset \mathrm{B}_{3} \oplus \mathrm{B}_{3} \supset \mathrm{B}_{3} \oplus \mathrm{G}_{2} \supset \mathrm{G}_{2} \oplus \mathrm{G}_{2} \supset \mathrm{G}_{2} \oplus \mathrm{A}_{1} \oplus \mathrm{A}_{1} \supset \mathrm{A}_{1} \oplus \mathrm{A}_{1} \oplus \mathrm{A}_{1}$

- $\mathrm{D}_{7} \supset \mathrm{B}_{3} \oplus \mathrm{B}_{3} \supset \mathrm{B}_{3} \oplus \mathbf{G}_{2} \supset \mathrm{G}_{2} \oplus \mathrm{G}_{2} \supset \mathrm{G}_{2} \oplus \mathrm{A}_{1} \oplus \mathrm{A}_{1} \supset A_{1} \oplus \mathrm{A}_{1} \oplus \mathrm{A}_{1} \oplus \mathrm{A}_{1}$

- $\mathrm{D}_{7} \supset \mathrm{B}_{3} \oplus \mathrm{B}_{3} \supset \mathrm{B}_{3} \oplus \mathrm{G}_{2} \supset \mathrm{G}_{2} \oplus \mathrm{G}_{2} \supset \mathrm{G}_{2} \oplus \mathrm{A}_{1} \oplus \mathrm{A}_{1} \supset \mathrm{A}_{2} \oplus \mathrm{A}_{1} \supset \mathrm{A}_{1} \oplus \mathrm{A}_{1} \oplus \mathrm{A}_{1}$

- $\mathrm{D}_{7} \supset \mathrm{B}_{3} \oplus \mathrm{B}_{3} \supset \mathrm{B}_{3} \oplus \mathrm{G}_{2} \supset \mathrm{G}_{2} \oplus \mathrm{G}_{2} \supset \mathrm{G}_{2} \oplus \mathrm{A}_{1} \oplus \mathrm{A}_{1} \supset \mathrm{A}_{2} \oplus \mathrm{A}_{1} \supset \mathrm{A}_{1}^{*} \oplus \mathrm{A}_{1} \oplus \mathrm{A}_{1}$

- $\mathrm{D}_{7} \supset \mathrm{B}_{3} \oplus \mathrm{B}_{3} \supset \mathrm{B}_{3} \oplus \mathrm{G}_{2} \supset \mathrm{A}_{1} \oplus \mathrm{A}_{1} \oplus \mathrm{A}_{1} \oplus \mathrm{G}_{2} \supset \mathrm{A}_{1} \oplus \mathrm{A}_{1} \oplus \mathrm{A}_{1} \oplus \mathrm{A}_{2}$

$$
\supset A_{1} \oplus A_{1} \oplus A_{1} \oplus A_{1}
$$

- $\mathrm{D}_{7} \supset \mathrm{B}_{3} \oplus \mathrm{B}_{3} \supset \mathrm{B}_{3} \oplus \mathrm{G}_{2} \supset \mathrm{A}_{1} \oplus \mathrm{A}_{1} \oplus \mathrm{A}_{1} \oplus \mathrm{G}_{2} \supset \mathrm{A}_{1} \oplus \mathrm{A}_{1} \oplus \mathrm{A}_{1} \oplus \mathrm{A}_{2}$

$$
\supset A_{1} \oplus A_{1} \oplus A_{1} \oplus A_{i}
$$

- $\mathrm{D}_{7} \supset \mathrm{B}_{3} \oplus \mathrm{B}_{3} \supset \mathrm{B}_{3} \oplus \mathrm{G}_{2} \supset \mathrm{A}_{1} \oplus \mathrm{A}_{1} \oplus \mathrm{A}_{1} \oplus \mathrm{G}_{2} \supset \mathrm{A}_{1} \oplus \mathrm{A}_{1} \oplus \mathrm{A}_{1} \oplus \mathrm{A}_{1}$

- $\mathrm{D}_{7} \supset \mathrm{B}_{3} \oplus \mathrm{B}_{3} \supset \mathrm{B}_{3} \oplus \mathrm{G}_{2} \supset \mathrm{A}_{1} \oplus \mathrm{A}_{1} \oplus \mathrm{A}_{1} \oplus \mathrm{G}_{2} \supset \mathrm{A}_{1} \oplus \mathrm{A}_{1} \oplus \mathrm{A}_{1} \oplus \mathrm{A}_{1} \oplus \mathrm{A}_{1}$

Das cadeias acima somente as que estāo em negrito sāo sobreviventes, enquanto que as demais são eliminadas, algumas por não apresentarem subespaços capazes de gerar os tripletos e 
sextupletos, outras por reproduzirem resultados já obtidos, e algumas por apresentarem mais que 4 subespaços de dimensão impar.

Sub-cadeias 10c:

- $\mathrm{D}_{7} \supset \mathrm{B}_{3} \oplus \mathrm{B}_{3} \supset \mathrm{B}_{3} \oplus \mathrm{A}_{1} \oplus \mathrm{A}_{1} \oplus \mathrm{A}_{1} \supset \mathrm{A}_{3} \oplus \mathrm{A}_{1} \oplus \mathrm{A}_{1} \oplus \mathrm{A}_{1} \supset \ldots$

- $\mathrm{D}_{7} \supset \mathrm{B}_{3} \oplus \mathrm{B}_{3} \supset \mathrm{B}_{3} \oplus \mathrm{A}_{1} \oplus \mathrm{A}_{1} \oplus \mathrm{A}_{1} \supset \mathrm{A}_{1} \oplus \mathrm{A}_{1} \oplus \mathrm{A}_{1} \oplus \mathrm{A}_{1} \oplus \mathrm{A}_{1}$

- $\mathrm{D}_{7} \supset \mathrm{B}_{3} \oplus \mathrm{B}_{3} \supset \mathrm{B}_{3} \oplus \mathrm{A}_{1} \oplus \mathrm{A}_{1} \oplus \mathrm{A}_{1} \supset \mathrm{G}_{2} \oplus \mathrm{A}_{1} \oplus \mathrm{A}_{1} \oplus \mathrm{A}_{1} \supset \ldots$

$\mathrm{Na}$ primeira cadeia as representações de $\mathrm{A}_{3}$ vem em pares conjugados, então elas são eliminadas por emparelhamento, na segunda cadeia não conseguimos gerar os tripletos e sextupletos, e na ultima devemos continuar a quebra, pois ela é sobrevivente:

- $\mathrm{D}_{7} \supset \mathrm{B}_{3} \oplus \mathrm{B}_{3} \supset \mathrm{B}_{3} \oplus \mathrm{A}_{1} \oplus \mathrm{A}_{1} \oplus \mathrm{A}_{1} \supset \mathrm{G}_{2} \oplus \mathrm{A}_{1} \oplus \mathrm{A}_{1} \oplus \mathrm{A}_{1}$ $\supset A_{2} \oplus A_{1} \oplus A_{1} \oplus A_{1} \supset A_{1} \oplus A_{1} \oplus A_{1} \oplus A_{1}$

- $\mathrm{D}_{7} \supset \mathrm{B}_{3} \oplus \mathrm{B}_{3} \supset \mathrm{B}_{3} \oplus \mathrm{A}_{1} \oplus \mathrm{A}_{1} \oplus \mathrm{A}_{1} \supset \mathrm{G}_{2} \oplus \mathrm{A}_{1} \oplus \mathrm{A}_{1} \oplus \mathrm{A}_{1}$ $\supset A_{2} \oplus A_{1} \oplus A_{1} \oplus A_{1} \supset A_{1}^{*} \oplus A_{1} \oplus A_{1} \oplus A_{1}$

- $\mathrm{D}_{7} \supset \mathrm{B}_{3} \oplus \mathrm{B}_{3} \supset \mathrm{B}_{3} \oplus \mathrm{A}_{1} \oplus \mathrm{A}_{1} \oplus \mathrm{A}_{1} \supset \mathrm{G}_{2} \oplus \mathrm{A}_{1} \oplus \mathrm{A}_{1} \oplus \mathrm{A}_{1} \supset \mathrm{A}_{1} \oplus \mathrm{A}_{1} \oplus \mathrm{A}_{1} \oplus \mathrm{A}_{1}$

- $\mathbf{D}_{7} \supset \mathbf{B}_{3} \oplus \mathbf{B}_{3} \supset \mathbf{B}_{3} \oplus \mathbf{A}_{1} \oplus \mathbf{A}_{1} \oplus \mathbf{A}_{1} \supset \mathbf{G}_{2} \oplus \mathbf{A}_{1} \oplus \mathbf{A}_{1} \oplus \mathbf{A}_{1}$

$$
\supset \mathbf{A}_{1} \oplus \mathbf{\mathbf { A } _ { 1 }} \oplus \mathbf{\mathbf { A } _ { 1 }} \oplus \mathbf{\mathbf { A } _ { 1 }} \oplus \mathbf{\mathbf { A } _ { 1 }}
$$

A última cadeia é sobrevivente e as demais são eliminadas por não gerarem os tripletos e sextupletos necessários.

Com isso finalizamos a análise das álgebras candidatas à reproduzirem a degenerescência do código genético, na primeira fase do processo de quebra de simetria, ou seja, quebra das cadeias até uma soma de $A_{1}$ 's, cujas cadeias sobreviventes foram:

- Cadeia 1: $\mathrm{C}_{2} \supset \mathrm{A}_{1} \oplus \mathrm{A}_{1}$

- Cadeia 2: $\mathrm{G}_{2} \supset \mathrm{A}_{1} \oplus \mathrm{A}_{1}$ 
- Cadeia 3: $C_{3} \supset C_{2} \oplus A_{1} \supset A_{1} \oplus A_{1}$

- Cadeia 4: $B_{6} \supset D_{6} \supset A_{1} \oplus C_{3} \supset A_{1} \oplus A_{1}$

- Cadeia 5: $C_{3} \supset C_{2} \oplus A_{1} \supset A_{1} \oplus A_{1} \oplus A_{1}$

- Cadeia 6: $B_{6} \supset D_{6} \supset A_{1} \oplus A_{1} \oplus A_{1}$

- Cadeia 7: $D_{7} \supset B_{3} \oplus B_{3} \supset B_{3} \oplus G_{2} \supset G_{2} \oplus G_{2} \supset G_{2} \oplus A_{1} \supset A_{1} \oplus A_{1} \oplus A_{1}$

- Cadeia 8: $D_{7} \supset A_{3} \oplus D_{4} \supset A_{3} \oplus A_{1} \oplus C_{2} \supset A_{3} \oplus A_{1} \oplus A_{1}$

$$
\supset A_{2} \oplus A_{1} \oplus A_{1} \supset A_{1} \oplus A_{1} \oplus A_{1}
$$

- Cadeia 9: $D_{7} \supset A_{1} \oplus A_{1} \oplus D_{5} \supset A_{1} \oplus A_{1} \oplus C_{2} \supset A_{1} \oplus A_{1} \oplus A_{1} \oplus A_{1}$

- Cadeia 10: $D_{7} \supset A_{1} \oplus A_{1} \oplus D_{5} \supset A_{1} \oplus A_{1} \oplus A_{4} \supset A_{1} \oplus A_{1} \oplus C_{2}$

$$
\supset A_{1} \oplus A_{1} \oplus A_{1} \oplus A_{1}
$$

- Cadeia 11: $D_{7} \supset A_{1} \oplus A_{1} \oplus D_{5} \supset A_{1} \oplus A_{1} \oplus A_{4} \supset A_{1} \oplus A_{1} \oplus A_{1} \oplus A_{2}$

$$
\supset A_{1} \oplus A_{1} \oplus A_{1} \oplus A_{1}
$$

- Cadeia 12: $D_{7} \supset A_{3} \oplus D_{4} \supset A_{3} \oplus A_{1} \oplus C_{2} \supset A_{3} \oplus A_{1} \oplus A_{1} \oplus A_{1}$

$$
\supset A_{2} \oplus A_{1} \oplus A_{1} \oplus A_{1} \supset A_{1} \oplus A_{1} \oplus A_{1} \oplus A_{1}
$$

- Cadeia 13: $D_{7} \supset B_{3} \oplus B_{3} \supset B_{3} \oplus G_{2} \supset G_{2} \oplus G_{2} \supset G_{2} \oplus A_{1} \oplus A_{1}$

$$
\supset A_{1} \oplus A_{1} \oplus A_{1} \oplus A_{1}
$$

- Cadeia 14: $D_{7} \supset B_{3} \oplus B_{3} \supset B_{3} \oplus A_{1} \oplus A_{1} \oplus A_{1} \supset G_{2} \oplus A_{1} \oplus A_{1} \oplus A_{1}$

$$
\supset A_{1} \oplus A_{1} \oplus A_{1} \oplus A_{1} \oplus A_{1}
$$

- Cadeia 15: $D_{7} \supset A_{3} \oplus D_{4} \supset A_{3} \oplus A_{1} \oplus A_{1} \oplus A_{1} \oplus A_{1} \supset A_{2} \oplus A_{1} \oplus A_{1} \oplus A_{1} \oplus A_{1}$ $\supset A_{1} \oplus A_{1} \oplus A_{1} \oplus A_{1} \oplus A_{1}$

Terminamos então a primeira fase da procura por simetrias no código genético. O próximo passo agora é a quebra da simetria su(2) ${ }^{p}$. Dessa forma podemos estabelecer um resultado, que é o primeiro resultado fundamental de nossa investigação. 
Não existe quebra de simetria padrão através de cadeias de subálgebras capaz de reproduzir exatamente as degenerescências do código genético.

À primeira vista, este resultado negativo parece ser um golpe fatal ao método algébrico para decifrar o código genético. Entretanto, como será mostrado mais a frente, existe uma generalização do procedimento de quebra de simetria discutido, que permite a chegar a uma resposta positiva. Ela é baseada na introdução de certos operadores associados às cadeias de subálgebras, semelhantes aos operadores de Casimir, e permite incorporar, com rigor matemático, o fenômeno de um congelamento (parcial) no processo de quebra de simetria durante o ultimo passo, de acordo com o congelamento na evolução do código genético postulado por biologistas e geneticistas (veja capítulo 2). 


\section{Capítulo 5}

\section{Quebra da Simetria su(2)}

Neste capítulo, estudaremos o que acontece quando o processo de quebra de simetria continua além de su(2), isto é, temos que quebrar uma ou várias subálgebras su(2) na subálgebra u(1). Essa quebra é feita usando operadores semelhantes aos operadores de Casimir (veja capítulo 3).

Dada uma álgebra de Lie semi-simples $g$ junto com uma cadeia de subálgebras semisimples $g_{1}, g_{2}, \ldots$,

$$
g \supset g_{1} \supset g_{2} \supset \ldots
$$

a distribuição de multipletos obtidos sucessivamente por decomposição de uma dada representação irredutível de $\boldsymbol{g}$ pode ser encontrada no espéctro de um simples operador $\boldsymbol{H}$ : ele pode ser definido como uma combinação linear genérica dos operadores de Casimir $C_{j}$ das subálgebras simples de $g$ que são as subálgebras semi-simples $g_{l}, g_{2}, \ldots$ que aparecem na cadeia:

$$
H=\sum_{j} \lambda_{j} C_{j}
$$

$\mathrm{Na}$ verdade, devido às relações de inclusão entre os $\boldsymbol{g}_{i}$, os operadores de Casimir $C_{j}$ auto-comutam, e a escolha genérica dos coeficientes $\lambda_{j}$ implica que os auto-espaços do 
operador $H$ coincidem com os auto-espaços comuns dos operadores de Casimir $C_{j}$, os quais po sua vez são os subespaços irredutíveis para a menor subálgebra (a última da cadeia).

Para sermos mais específicos, lembramos que depois da primeira fase do processo de quebra de simetria ter sido completada, a última subálgebra na cadeia é uma soma direta de subálgebras sú(2), tal que a Hamiltoniana $H$ associada a este estágio pode ser escrita na forma

$$
H=\sum_{j} \lambda_{j} C_{j}+\sum_{k=1}^{p} \alpha_{k} L_{k}^{2},
$$

onde $p$, o número total de subálgebras su(2) que aparecem no final da cadeia, varia entre 1 e o posto de $g$, dependendo da cadeia. Os $C_{j}$ são agora os operadores de Casimir associados às subálgebras simples diferentes de $\operatorname{su}(2)$, enquanto que $L_{k}^{2}=L_{k, x}^{2}+L_{k, y}^{2}+L_{k, z}^{2}$ é o operador de Casimir padrão da $k$-ésima subálgebra su(2) $(1 \leq k \leq p)$.

A segunda fase, a qual envolve quebra de uma ou de várias das subálgebras su(2), será implementada por uma Hamiltoniana da forma

$$
H=\sum_{j} \lambda_{j} C_{j}+\sum_{k=1}^{p} \alpha_{k} L_{k}^{2}+\sum_{k=1}^{p} \beta_{k} L_{k, z}^{2}+\sum_{k=1}^{p} \gamma_{k} L_{k, z}
$$

Para explicar o efeito dos novos termos, consideramos o simples caso de um único su(2). Levando em conta o fato de que as representações irredutíveis de su(2), caracterizadas pelo spin $s$ (correspondente ao peso máximo $2 s$ ), formam espaços de dimensão $(2 s+1)$ nos quais o operador de Casimir padrão do su(2), $L^{2}$, assume os valores $s(s+1)$, enquanto que o operador $L_{2}$ tem $2 s+1$ autovalores distintos, temos que:

a) o operador $L_{z}$ proporciona uma completa divisão de um multipleto de dimensão $(2 s+1) \mathrm{em}$ $2 s+1$ singletos, enquanto que,

b) o operador $L_{z}^{2}$ proporciona uma suave divisão de um multipleto de dimensão $(2 s+1)$ em $s$ dubletos e um singleto se sé inteiro, ou $s$ dubletos se $s$ é semi-inteiro.

Somente a primeira possibilidade corresponde a uma quebra de simetria autêntica a nível de álgebras de Lie: da álgebra de Lie su(2) para a subálgebra maximal u(1). Observou-se ${ }^{23}$ que 
ambas as possibilidades levam em conta uma interpretação natural em termos de uma quebra de simetria autêntica a nível de grupos de Lie, ou seja, do grupo correspondente SU(2) para:

a) seu subgrupo maximal $\mathrm{U}(1) \cong \mathrm{SO}(2)$, ou

b) seu subgrupo maximal $Z_{2} \times U(1) \cong O(2)$, um subgrupo de $S U(2)$ formado por dois círculos:

$$
Z_{2} \times U(1)=\left\{\left(\begin{array}{cc}
e^{i \alpha} & 0 \\
0 & e^{-i \alpha}
\end{array}\right) / \alpha \in R\right\} \cup\left\{\left(\begin{array}{cc}
0 & e^{i \beta} \\
e^{-i \beta} & 0
\end{array}\right) / \beta \in R\right\} .
$$

Note que $Z_{2} \times U(1)$ é gerado por $U(1)$ junto com a matriz,

$$
\left(\begin{array}{ll}
0 & 1 \\
1 & 0
\end{array}\right)
$$

que é o gerador do grupo de Weyl de SU(2). Por abuso de notação nos referimos a estas duas reduções da simetria su(2), em forma abreviada, como simetria so(2) e simetria o(2), respectivamente (ainda que os termos "simetria $\mathrm{SO}(2)$ " e "simetria $\mathrm{O}(2)$ " sejam mais apropriadas).

Outro fato importante no processo de quebra de simetria é que os coeficientes $\gamma_{\mathrm{k}}$ da Hamiltoniana $H$ sejam polinômios nos operadores de Casimir $L_{k}^{2}$ das subálgebras su(2), em vez de constantes. Isto possibilita uma interrupção no processo de quebra de simetria no último passo, porque multipletos do penúltimo estagio que normalmente se dividem no último passo não serão quebrados se as suas etiquetas sob os su(2) são tais que o correspondente coeficiente $\gamma$ desaparece. Assim uma interrupção parcial nas diferenciações do código genético está de acordo com seu congelamento na presente forma, proposta por biologistas e geneticistas (veja capítulo 2), supondo-se que este fenômeno deve ocorrer exclusivamente durante o último passo da quebra de simetria. Veremos mais adiante exemplos concretos para um melhor entendimento dessa discussão.

Discutiremos agora a segunda fase do processo de quebra de simetria, durante a qual uma ou varias das subálgebras su(2) são quebradas. Como mencionamos antes, isso pode ser feito de duas maneiras: para o(2) ou para so(2). No segundo caso, a degenerescência é totalmente removida, enquanto que no primeiro caso as representaçōes de dimensões pares 
(spin semi-inteiro) de su(2) produzem somente dubletos e as representações de dimensões impares (spin inteiro) de su(2) produzem uma coleção de dubletos mais um singleto. Representamos, nas tabelas abaixo, os singletos por $2 m(m=-s, \ldots, s)$ e os dubletos por $\pm 2 m$ $(m=0, \ldots, s$, com $m>0)$, onde $m$ é o número quântico magnético.

Inicialmente, introduzimos um novo critério que permite mais uma redução significativa do número de cadeias a serem analisadas. Ele é baseado na observação de que durante a segunda fase, a quebra de simetria não pode gerar um multipleto com dimensão divisível por 3 a partir de um multipleto (maior) com dimensão indivisível por 3. Portanto, é conveniente definir o seguinte número de trialidade, associado a cada estágio durante a fase 2 :

$\mathrm{d}_{3}=$ soma das dimensões de todos os multipletos com dimensão divisível por 3.

Com esta notação, a afirmação é que quando prosseguimos ao longo de cada cadeia, o número de trialidade $d_{3}$ não pode crescer, durante a fase 2 . Como o valor de $d_{3}$ na distribuição final dos multipletos no código genético é 24 , chegamos à conclusão de que qualquer cadeia sobrevivente que, em algum ponto durante a fase 2 do processo de quebra de simetria anterior ao último passo, chega a ferir a estimativa

- $\mathrm{d}_{3} \geq 24$

não será capaz de gerar os sextupletos e tripletos do código genético e, portanto, pode ser descartada. $\mathrm{O}$ critério não se aplica diretamente ao último passo, devido à possibilidade de interromper a perda de multipletos com dimensão divisível por 3, através de um congelamento de tais multipletos, e portanto o critério será na maioria dos casos aplicado aos passos anteriores que podem, por exemplo, ser identificados pela simples condição de que o número total de multipletos ainda não alcançou o valor crítico, 21.

Outra observação, de natureza semelhante, é que pelo menos um su(2) deve permanecer sem quebra ou, se quebra, deve quebrar apenas no último passo e com congelamento, para preservar os multipletos com dimensão divisível por 3 e assim manter a capacidade de gerar os sextupletos e tripletos do código genético. De fato, se todas as subálgebras su(2) quebram em o(2) ou em so(2), sem congelamento, a multiplicidade de todos os multipletos será uma potência de dois. Isso é uma forte restrição aos possíveis esquemas de quebra de simetria da segunda fase: entre outras coisas, elimina todas as cadeias que terminam em um único su(2). 
Existem outras estratégias gerais para reduzir o número de casos a serem analisados, em particular as seguintes:

1. Se na tabela resultante da fase 1 aparecem representações de spin 2 (dimensão 5) ou de spin $\geq 3$ (dimensão $\geq 7$ ), com respeito a algum $A_{1}$, então este $A_{1}$ tem que ser quebrado, no mínimo em $L_{i, z}^{2}$ e talvez até em $L_{i, z}$. Tais quebras serão executadas em primeiro lugar.

2. Se na tabela resultante da fase 1 aparecem só representações de spin 0 (dimensão 1) e de spin $1 / 2$ (dimensão 2 ), com respeito a algum $A_{1}$, digamos o $i$-ésimo, então podemos desconsiderar a quebra em $L_{i, 2}^{2}$, pois ela não produzirá nenhuma quebra; podemos nos restringir à quebra em $L_{i, z}$.

3. Se a tabela resultante da fase 1 é simétrica com respeito a certas permutações entre os $A_{1}$ 's, esta simetria pode ser usada para eliminar certas quebras que não dão nada de novo, comparando com as outras já estudadas.

Veremos em seguida como aplicar estes argumentos, em casos específicos.

\subsection{Análise das Cadeias de $C_{2}, G_{2}$ e $C_{3}$ na Fase 2}

Com as estratégias acima expostas à disposição, vamos investigar a seguir as cadeias sobreviventes originando das álgebras de posto baixo, $\mathrm{C}_{2}, \mathrm{G}_{2}$ e $\mathrm{C}_{3}$. No final da fase 1 , nenhuma destas cadeias atingiu o valor crítico de 21 multipletos; e portanto o processo de quebra deve prosseguir para a fase 2. Há 2 cadeias que terminaram em uma única cópia de $\operatorname{su}(2)\left(C_{2} \supset A_{1}\right.$ e $\left.C_{3} \supset A_{1}\right)$ e uma cadeia que terminou na soma direta de duas cópias de $\operatorname{su}(2)\left(C_{3} \supset A_{1} \oplus A_{1}\right)$ com $d_{3}=18$ : elas podem ser eliminadas imediatamente pois serão incapazes de gerar os sextupletos e tripletos do código genético. Ademais, há 3 cadeias que terminaram na soma direta de duas cópias de su(2) e uma cadeia que terminou na soma direta de três cópias de su(2) com $d_{3} \geq 24$ : essas precisam ser analisadas em mais detalhe.

Cadeia I: $\mathrm{sp}(4) \supset \mathrm{su}(2) \oplus \mathrm{su}(2)$ ou $\mathrm{C}_{2} \supset \mathrm{A}_{1} \oplus \mathrm{A}_{1}$.

Nesta cadeia, a primeira $e$ a segunda álgebra $\mathrm{A}_{1}$ tem que ser quebradas, para eliminar as representações de dimensão 10 , pois não existem códons com degenerescência 5 ou 10. Note também a simetria da tabela resultante da fase 1 com respeito à troca das duas álgebras $\mathrm{A}_{1}$. Mas 
se quebramos em $L_{1.2}^{2}$ ou $L_{2.2}^{2}$, que é a quebra mais suave, geramos somente 14 multipletos com $\mathrm{d}_{3}=18$ (veja a tabela 10 ), o que elimina qualquer possibilidade de prosseguir.

\begin{tabular}{|c|c|c|c|}
\hline $\mathrm{A}_{1} \oplus \mathrm{A}_{1}$ & $\mathrm{~d}$ & $L_{1,2}^{2}$ & $\mathrm{~d}$ \\
\hline$(3,2)$ & \multirow{2}{*}{12} & $( \pm 3,2)$ & 6 \\
\hline & & $( \pm 1,2)$ & 6 \\
\hline$(2,3)$ & 12 & $( \pm 2,3)$ & 8 \\
\hline & & $(0,3)$ & 4 \\
\hline$(4,1)$ & \multirow{2}{*}{10} & $( \pm 4,1)$ & 4 \\
\cline { 3 - 4 } & & $( \pm 2,1)$ & 4 \\
\hline & & $(0,1)$ & 2 \\
\hline$(1,4)$ & 10 & $( \pm 1,4)$ & 10 \\
\hline$(2,1)$ & 6 & $( \pm 2,1)$ & 4 \\
\hline & & $(0,1)$ & 2 \\
\hline$(1,2)$ & 6 & $( \pm 1,2)$ & 6 \\
\hline$(3,0)$ & 4 & $( \pm 3,0)$ & 2 \\
\cline { 3 - 4 } & & $( \pm 1,0)$ & 2 \\
\hline$(0,3)$ & 4 & $(0,3)$ & 4 \\
\hline 8 subespaços & \multicolumn{2}{|c|}{14 subespaços } \\
\hline $\mathrm{d}_{3}=36$ & \multicolumn{3}{|c}{$\mathrm{d}_{3}=18$} \\
\hline
\end{tabular}

Tabela 10: Quebra da representação tipo códon de sp(4) na cadeia

$$
\mathrm{sp}(4) \supset \mathrm{su}(2) \oplus \mathrm{su}(2) \supset \mathrm{o}(2) \oplus \mathrm{su}(2) .
$$

Cadeia 2: $G_{2} \supset \operatorname{su}(2) \oplus \operatorname{su}(2)$ ou $G_{2} \supset A_{1} \oplus A_{1}$.

Nesta cadeia, a segunda álgebra $A_{1}$ tem que ser quebrada, para eliminar as representaçōes de dimensão 10 , pois não existem códons com degenerescência 5 ou 10 . Se quebramos em $L_{2,2}^{2}$, que é a quebra mais suave, geramos 17 multipletos com $d_{3}=24$ (veja as tabelas 11a e 11b), e a quebra deve prosseguir. Quebrando em seguida em $L_{1.2}^{2}$, geramos 23 multipletos com $d_{3}=0$ (veja a tabela 11a), mas teremos que quebrar o octupleto e congelar os três sextupletos e dois tripletos, o que fornece 18 multipletos com três sextupletos, oito quadrupletos, dois tripletos, três dubletos e dois singletos - próximo ao código genético. Quebrando em seguida em $L_{2.2}$, geramos 30 multipletos com $d_{3}=24$ (veja a tabela 11 b). Neste 
caso, os octupletos devem ser quebrados, enquanto que os três sextupletos e dois tripletos devem ser congelados. Se quebramos três dos seis quadrupletos e congelamos os outros três, conforme indicado em negrito na tabela, obtemos exatamente a degenerescência do código genético, como já foi observado ${ }^{23}$.

\begin{tabular}{|c|c|c|c|c|c|}
\hline $\mathrm{A}_{1} \oplus \mathrm{A}_{1}$ & $\mathrm{~d}$ & $L_{2, z}^{2}$ & $\mathrm{~d}$ & $\left(L_{1,2}^{2}, L_{2,2}^{2}\right)$ & d \\
\hline \multirow[t]{6}{*}{$(2,4)$} & \multirow[t]{6}{*}{15} & \multirow[t]{2}{*}{$(2, \pm 4)$} & \multirow[t]{2}{*}{6} & $( \pm 2, \pm 4)$ & 4 \\
\hline & & & & $(0, \pm 4)$ & 2 \\
\hline & & \multirow[t]{2}{*}{$(2, \pm 2)$} & \multirow[t]{2}{*}{6} & $( \pm 2, \pm 2)$ & 4 \\
\hline & & & & $(0, \pm 2)$ & 2 \\
\hline & & \multirow[t]{2}{*}{$(2,0)$} & \multirow[t]{2}{*}{3} & $( \pm 2,0)$ & 2 \\
\hline & & & & $(0,0)$ & 1 \\
\hline \multirow[t]{3}{*}{$(1,5)$} & \multirow[t]{3}{*}{12} & $(1, \pm 5)$ & 4 & $( \pm 1, \pm 5)$ & 4 \\
\hline & & $(1, \pm 3)$ & 4 & $( \pm 1, \pm 3)$ & 4 \\
\hline & & $(1, \pm 1)$ & 4 & $( \pm 1, \pm 1)$ & 4 \\
\hline \multirow[t]{4}{*}{$(2,2)$} & \multirow[t]{4}{*}{9} & \multirow[t]{2}{*}{$(2, \pm 2)$} & \multirow[t]{2}{*}{6} & $( \pm 2, \pm 2)$ & 4 \\
\hline & & & & $(0, \pm 2)$ & 2 \\
\hline & & \multirow[t]{2}{*}{$(2,0)$} & \multirow[t]{2}{*}{3} & $( \pm 2,0)$ & 2 \\
\hline & & & & $(0,0)$ & 1 \\
\hline \multirow[t]{2}{*}{$(3,1)$} & \multirow[t]{2}{*}{8} & \multirow[t]{2}{*}{$(3, \pm 1)$} & \multirow[t]{2}{*}{8} & $( \pm 3, \pm 1)$ & 4 \\
\hline & & & & $( \pm 1, \pm 1)$ & 4 \\
\hline \multirow[t]{2}{*}{$(1,3)$} & \multirow[t]{2}{*}{8} & $(1, \pm 3)$ & 4 & $( \pm 1, \pm 3)$ & 4 \\
\hline & & $(1, \pm 1)$ & 4 & $( \pm 1, \pm 1)$ & 4 \\
\hline \multirow[t]{3}{*}{$(0,4)$} & \multirow[t]{3}{*}{5} & $(0, \pm 4)$ & 2 & $(0, \pm 4)$ & 2 \\
\hline & & $(0, \pm 2)$ & 2 & $(0, \pm 2)$ & 2 \\
\hline & & $(0,0)$ & 1 & $(0,0)$ & 1 \\
\hline$(1,1)$ & 4 & $(1, \pm 1)$ & 4 & $( \pm 1, \pm 1)$ & 4 \\
\hline \multirow[t]{2}{*}{$(0,2)$} & \multirow[t]{2}{*}{3} & $(0, \pm 2)$ & 2 & $(0, \pm 2)$ & 2 \\
\hline & & $(0,0)$ & 1 & $(0,0)$ & 1 \\
\hline \multicolumn{2}{|c|}{8 subespaços } & \multicolumn{2}{|c|}{17 subespaços } & \multicolumn{2}{|c|}{23 subespaços } \\
\hline \multicolumn{2}{|c|}{$\mathrm{d}_{3}=39$} & \multicolumn{2}{|c|}{$\mathrm{d}_{3}=24$} & \multicolumn{2}{|c|}{$\mathrm{d}_{3}=0$} \\
\hline
\end{tabular}

Tabela 11a: Quebra da representação tipo códon de $\mathrm{G}_{2}$ na cadeia

$$
\mathrm{G}_{2} \supset \mathrm{su}(2) \oplus \mathrm{su}(2) \supset \mathrm{su}(2) \oplus \mathrm{o}(2) \supset \mathrm{o}(2) \oplus \mathrm{o}(2) .
$$




\begin{tabular}{|c|c|c|c|c|c|}
\hline $\mathrm{A}_{1} \oplus \mathrm{A}_{1}$ & d & $L_{2, z}^{2}$ & d & $L_{2, z}$ & $\mathrm{~d}$ \\
\hline \multirow[t]{5}{*}{$(2,4)$} & \multirow[t]{5}{*}{15} & \multirow[t]{2}{*}{$(2, \pm 4)$} & \multirow[t]{2}{*}{6} & $(2,+4)$ & 3 \\
\hline & & & & $(2,-4)$ & 3 \\
\hline & & \multirow[t]{2}{*}{$(2, \pm 2)$} & \multirow[t]{2}{*}{6} & $(2,+2)$ & 3 \\
\hline & & & & $(2,-2)$ & 3 \\
\hline & & $(2,0)$ & 3 & $(2,0)$ & 3 \\
\hline \multirow[t]{6}{*}{$(1,5)$} & \multirow[t]{6}{*}{12} & \multirow[t]{2}{*}{$(1, \pm 5)$} & \multirow[t]{2}{*}{4} & $(1,+5)$ & 2 \\
\hline & & & & $(1,-5)$ & 2 \\
\hline & & \multirow[t]{2}{*}{$(1, \pm 3)$} & \multirow[t]{2}{*}{4} & $(1,+3)$ & 2 \\
\hline & & & & $(1,-3)$ & 2 \\
\hline & & \multirow[t]{2}{*}{$(1, \pm 1)$} & \multirow[t]{2}{*}{4} & $(1,+1)$ & 2 \\
\hline & & & & $(1,-1)$ & 2 \\
\hline \multirow[t]{3}{*}{$(2,2)$} & \multirow[t]{3}{*}{9} & \multirow[t]{2}{*}{$(2, \pm 2)$} & \multirow[t]{2}{*}{6} & $(2,+2)$ & 3 \\
\hline & & & & $(2,-2)$ & 3 \\
\hline & & $(2,0)$ & 3 & $(2,0)$ & 3 \\
\hline \multirow[t]{2}{*}{$(3,1)$} & \multirow[t]{2}{*}{8} & \multirow[t]{2}{*}{$(3, \pm 1)$} & \multirow[t]{2}{*}{8} & $(3,+1)$ & 4 \\
\hline & & & & $(3,-1)$ & 4 \\
\hline \multirow[t]{4}{*}{$(1,3)$} & \multirow[t]{4}{*}{8} & \multirow[t]{2}{*}{$(1, \pm 3)$} & \multirow[t]{2}{*}{4} & $(1,+3)$ & 2 \\
\hline & & & & $(1,-3)$ & 2 \\
\hline & & \multirow[t]{2}{*}{$(1, \pm 1)$} & \multirow[t]{2}{*}{4} & $(1,+1)$ & 2 \\
\hline & & & & $(1,-1)$ & 2 \\
\hline \multirow[t]{5}{*}{$(0,4)$} & \multirow[t]{5}{*}{5} & $(0, \pm 4)$ & 2 & $(0,+4)$ & 1 \\
\hline & & & & $(0,-4)$ & 1 \\
\hline & & $(0, \pm 2)$ & 2 & $(0,+2)$ & 1 \\
\hline & & & & $(0,-2)$ & 1 \\
\hline & & $(0,0)$ & 1 & $(0,0)$ & 1 \\
\hline$(1,1)$ & 4 & $(1, \pm 1)$ & 4 & $(1,+1)$ & 2 \\
\hline & & & & $(1,-1)$ & 2 \\
\hline$(0,2)$ & 3 & $(0, \pm 2)$ & 2 & $(0,+2)$ & 1 \\
\hline & & & & $(0,-2)$ & 1 \\
\hline & & $(0,0)$ & 1 & $(0,0)$ & 1 \\
\hline 8 subesp & & 17 subes & & 30 sube & \\
\hline $\mathrm{d}_{3}=3$ & & $\mathrm{~d}_{3}=$ & & $\mathrm{d}_{3}=$ & \\
\hline
\end{tabular}

Tabela 11b: Quebra da representação tipo códon de $G_{2}$ na cadeia

$$
\mathrm{G}_{2} \supset \mathrm{su}(2) \oplus \mathrm{su}(2) \supset \mathrm{su}(2) \oplus \mathrm{o}(2) \supset \mathrm{su}(2) \oplus \mathrm{so}(2) .
$$


Cadeia 3: $\mathrm{sp}(6) \supset \mathrm{sp}(4) \oplus \mathrm{su}(2) \supset \mathrm{su}(2) \oplus \mathrm{su}(2)$ ou $\mathrm{C}_{3} \supset \mathrm{C}_{2} \oplus \mathrm{A}_{1} \supset \mathrm{A}_{1} \oplus \mathrm{A}_{1}$.

Nesta cadeia, a primeira álgebra $A_{1}$ tem que ser quebrada, para eliminar as representações de dimensão 8,10 and 14, pois não existem códons com degenerescência 5 ou 7 ou 10 ou 14. Se quebramos em $L_{1,2}^{2}$, que é a quebra mais suave, geramos 22 multipletos com $\mathrm{d}_{3}=12$ (veja a tabela 12), e a quebra deve terminar. Mesmo levando em conta a possibilidade de congelamento, este esquema não fornece tripletos ou singletos.

\begin{tabular}{|c|c|c|c|}
\hline$A_{1} \oplus A_{1}$ & d & $L_{1, z}^{2}$ & d \\
\hline \multirow[t]{4}{*}{$(6,1)$} & \multirow[t]{4}{*}{14} & $( \pm 6,1)$ & 4 \\
\hline & & $( \pm 4,1)$ & 4 \\
\hline & & $( \pm 2,1)$ & 4 \\
\hline & & $(0,1)$ & 2 \\
\hline \multirow{2}{*}{$(2,1)$} & \multirow[t]{2}{*}{6} & $( \pm 2,1)$ & 4 \\
\hline & & $(0,1)$ & 2 \\
\hline \multirow[t]{4}{*}{$(7,0)$} & \multirow[t]{4}{*}{8} & $( \pm 7,0)$ & 2 \\
\hline & & $( \pm 5,0)$ & 2 \\
\hline & & $( \pm 3,0)$ & 2 \\
\hline & & $( \pm 1,0)$ & 2 \\
\hline \multirow[t]{3}{*}{$(5,0)$} & \multirow[t]{3}{*}{6} & $( \pm 5,0)$ & 2 \\
\hline & & $( \pm 3,0)$ & 2 \\
\hline & & $( \pm 1,0)$ & 2 \\
\hline$(1,0)$ & 2 & $( \pm 1,0)$ & 2 \\
\hline \multirow[t]{2}{*}{$(3,2)$} & \multirow[t]{2}{*}{12} & $( \pm 3,2)$ & 6 \\
\hline & & $( \pm 1,2)$ & 6 \\
\hline \multirow[t]{3}{*}{$(4,1)$} & \multirow[t]{3}{*}{10} & $( \pm 4,1)$ & 4 \\
\hline & & $( \pm 2,1)$ & 2 \\
\hline & & $(0,1)$ & 2 \\
\hline \multirow[t]{2}{*}{$(3,0)$} & \multirow[t]{2}{*}{4} & $( \pm 3,0)$ & 2 \\
\hline & & $( \pm 1,0)$ & 2 \\
\hline$(0,3)$ & 4 & $(0,3)$ & 4 \\
\hline \multicolumn{2}{|c|}{9 subespaços } & \multicolumn{2}{|c|}{22 subespaços } \\
\hline \multicolumn{2}{|c|}{$\mathrm{d}_{3}=24$} & \multicolumn{2}{|c|}{$\mathrm{d}_{3}=12$} \\
\hline
\end{tabular}

Tabela 12: Quebra da representação tipo códon de sp(6) na cadeia $\mathrm{sp}(6) \supset \mathrm{sp}(4) \oplus \mathrm{su}(2) \supset \mathrm{su}(2) \oplus \mathrm{su}(2) \supset \mathrm{o}(2) \oplus \mathrm{su}(2)$. 
Finalmente voltamos para a única cadeia sobrevivente finalizada na soma direta de três cópiaas de su(2):

Cadeia 4: $\mathrm{sp}(6) \supset \mathrm{sp}(4) \oplus \mathrm{su}(2) \supset \mathrm{su}(2) \oplus \mathrm{su}(2) \oplus \mathrm{su}(2)$ ou

$$
\mathrm{C}_{3} \supset \mathrm{C}_{2} \oplus \mathrm{A}_{1} \supset \mathrm{A}_{1} \oplus \mathrm{A}_{1} \oplus \mathrm{A}_{1} \text {. }
$$

Nesta cadeia, uma das três álgebras $A_{1}$ tem que ser quebrada, para eliminar os octupletos. Note também a simetria da tabela resultante da fase 1 com respeito à qualquer permutação das três álgebras $A_{1}$, que simplifica a análise. As possibilidades de uma futura quebra de simetria podem então ser organizadas como abaixo.

1. Quebrando uma ou duas das subálgebras su(2) para o(2) deixará os octupletos, quadrupletos e dubletos invariantes e quebrará dois ou quatro dos sextupletos em quadrupletos mais dubletos, gerando 16 ou 18 subespaços, respectivamente. Dessa forma a quebra de simetria deve proceder além deste ponto: pelo menos uma das subálgebras su(2) deve ser quebrada para so(2). Por outro lado, neste estágio devem permanecer pelo menos três sextupletos, pois congelamento é permitido somente no último passo; portanto, apenas uma das subálgebras su(2) pode ser quebrada para o(2), gerando dois octupletos, quatro sextupletos, dois quadrupletos e oito dubletos.

2. Quebrando uma das subálgebras su(2) diretamente para so(2)

(a) quebra os dois octupletos em quatro quadrupletos,

(b) deixa dois dos seis sextupletos invariantes, quebra dois deles em quatro tripletos e os dois restantes em seis dubletos,

(c) deixa quatro dos seis dubletos invariantes e quebra os dois restantes em quatro singletos,

gerando um total de 24 subespaços. Portanato, a quebra de simetria deve parar neste ponto. Mas se permitirmos o congelamento de um dos sextupletos quebrados em tripletos e um dos dubletos quebrados em singletos, para evitar o aparecimento de mais que dois tripletos ou singletos, não existe um caminho para gerar um quinto quadrupleto: temos somente 4 quadrupletos (ao invés de 5) e 11.dubletos (ao invés de 9). Dessa forma, essas cadeias são eliminadas. 
3. Quebrando uma das subálgebras su(2) para o(2) no primeiro passo e depois para so(2) em um segundo passo, resultará em um esquema de quebra ligeiramente diferente. Resumindo:

(a) os dois octupletos quebram em quatro quadrupletos,

(b) dois dos quatro sextupletos permanecem invariantes, enquanto que os outros dois quebram em quatro tripletos, então um desses dois devem ser congelados,

(c) os dois quadrupletos quebram em quatro dubletos, então um deles deve ser congelado,

(d) seis dos oito dubletos permanecem invariantes, enquanto que os outros dois quebram em quatro singletos, então um desses dois deve ser congelado.

O problema com este esquema é que os dois dubletos quebrados em singletos pertencem à mesma representação irredutível de $\operatorname{su}(2) \oplus \mathrm{su}(2) \oplus \mathrm{su}(2)$ : elas se diferenciam somente pelo peso máximo da representação de $\mathrm{sp}(4) \oplus \mathrm{su}(2)$ da qual originaram. A necessidade de congelar uma delas e de permitir quebrar a outra é inconsistente com o critério para termos de congelamento aceitáveis, então essas cadeias também devem ser eliminadas.

4. Existe agora somente uma possibilidade, a qual consiste em quebrar uma das subálgebras su(2) (digamos, a segunda) para o(2) em um primeiro passo e depois quebrar uma das outros (digamos, a terceira) para so(2) em um segundo passo. Isto dá origem ao esquema apresentado na tabela 13. Como já foi observado nas referências ${ }^{8,22}$, este reproduz exatamente a degenerescência do código genético, proporcionando que os dois sextupletos que quebrariam em dubletos e os dois dubletos que quebrariam em singletos sejam congelados. Novamente, esta interrupção está indicada em negrito na tabela 13. 


\begin{tabular}{|c|c|c|c|c|c|}
\hline$A_{1} \oplus A_{1} \oplus A_{1}$ & d & $L_{2, z}^{2}$ & d & $\left(L_{2, z}^{2}, L_{3, z}\right)$ & d \\
\hline \multirow[t]{2}{*}{$(1,1,1)$} & \multirow[t]{2}{*}{8} & \multirow[t]{2}{*}{$(1, \pm 1,1)$} & \multirow[t]{2}{*}{8} & $(1, \pm 1,+1)$ & 4 \\
\hline & & & & $(1, \pm 1,-1)$ & 4 \\
\hline \multirow[t]{2}{*}{$(2,0,1)$} & \multirow[t]{2}{*}{6} & \multirow[t]{2}{*}{$(2,0,1)$} & \multirow[t]{2}{*}{6} & $(2,0,+1)$ & 3 \\
\hline & & & & $(2,0,-1)$ & 3 \\
\hline \multirow[t]{4}{*}{$(0,2,1)$} & \multirow[t]{4}{*}{6} & \multirow[t]{2}{*}{$(0, \pm 2,1)$} & \multirow[t]{2}{*}{4} & $(0, \pm 2),+1)$ & 2 \\
\hline & & & & $(0, \pm 2,-1)$ & 2 \\
\hline & & \multirow[t]{2}{*}{$(0,0,1)$} & \multirow[t]{2}{*}{2} & $(0,0,(+1))$ & 1 \\
\hline & & & & $(0,0,(-1))$ & 1 \\
\hline$(2,1,0)$ & 6 & $(2, \pm 1,0)$ & 6 & $(2, \pm 1,0)$ & 6 \\
\hline \multirow[t]{2}{*}{$(1,2,0)$} & \multirow[t]{2}{*}{6} & $(1,+2,0)$ & 4 & $(1, \pm 2,0)$ & 4 \\
\hline & & $(1,0,0)$ & 2 & $(1,0,0)$ & 2 \\
\hline$(1,0,0)$ & 2 & $(1,0,0)$ & 2 & $(1,0,0)$ & 2 \\
\hline$(0,1,0)$ & 2 & $(0, \pm 1,0)$ & 2 & $(0, \pm 1,0)$ & 2 \\
\hline \multirow[t]{3}{*}{$(1,0,2)$} & \multirow[t]{3}{*}{6} & \multirow[t]{3}{*}{$(1,0,2)$} & \multirow[t]{3}{*}{6} & $(1,0,+2)$ & $\overline{2}$ \\
\hline & & & & $(1,0,-2)$ & 2 \\
\hline & & & & $(1,0,0)$ & 2 \\
\hline \multirow[t]{3}{*}{$(0,1,2)$} & \multirow[t]{3}{*}{6} & \multirow[t]{3}{*}{$(0, \pm 1,2)$} & \multirow[t]{3}{*}{6} & $(0, \pm 1,+2)$ & 2 \\
\hline & & & & $(0, \pm 1,-2)$ & 2 \\
\hline & & & & $(0, \pm 1,0)$ & 2 \\
\hline \multirow[t]{2}{*}{$(1,1,1)$} & \multirow[t]{2}{*}{8} & \multirow[t]{2}{*}{$(1, \pm 1,1)$} & \multirow[t]{2}{*}{8} & $(1, \pm 1,+2)$ & 4 \\
\hline & & & & $(1, \pm 1,-2)$ & 4 \\
\hline \multirow[t]{2}{*}{$(0,0,1)$} & \multirow[t]{2}{*}{2} & \multirow[t]{2}{*}{$(0,0,1)$} & \multirow[t]{2}{*}{2} & $(0,0,+1)$ & 1 \\
\hline & & & & $(0,0,-1)$ & 1 \\
\hline$(1,0,0)$ & 2 & $(1,0,0)$ & 2 & $(1,0,0)$ & 2 \\
\hline$(0,1,0)$ & 2 & $(0, \pm 1,0)$ & 2 & $(0, \pm 1,0)$ & 2 \\
\hline$(0,0,1)$ & 2 & $(0,0,1)$ & 2 & $(0,0,+1)$ & 1 \\
\hline & & & & $(0,0,-1)$ & 1 \\
\hline
\end{tabular}

Tabela 13: Quebra da representação tipo códon de sp(6) na cadeia $\mathrm{sp}(6) \supset \mathrm{sp}(4) \oplus \mathrm{su}(2) \supset \mathrm{su}(2) \oplus \mathrm{su}(2) \oplus \mathrm{su}(2) \supset \mathrm{su}(2) \oplus \mathrm{o}(2) \oplus \mathrm{su}(2) \supset \mathrm{su}(2) \oplus \mathrm{o}(2) \oplus \mathrm{so}(2)$.

Com este resultado, nossa procura por simetrias usando álgebras de Lie simples de posto baixo está completa. Identificamos duas cadeias como as que reproduzem a degenerescência do código genético, que são:

- $\mathrm{G}_{2} \supset \mathrm{su}(2) \oplus \mathrm{su}(2) \supset \mathrm{su}(2) \oplus \mathrm{o}(2) \supset \mathrm{su}(2) \oplus \mathrm{so}(2)^{\mathrm{f}}$, 
- $\mathrm{sp}(6) \supset \mathrm{sp}(4) \oplus \mathrm{su}(2) \supset \mathrm{su}(2) \oplus \mathrm{su}(2) \oplus \mathrm{su}(2)$

$$
\supset \mathrm{su}(2) \oplus \mathrm{o}(2) \oplus \mathrm{su}(2) \supset \mathrm{su}(2) \oplus \mathrm{o}(2) \oplus \mathrm{so}(2)^{\mathrm{f}},
$$

onde o índice " $\mathrm{f}$ " indica o fato de que a ultima quebra é somente parcial devido ao fenômeno do congelamento, o qual pode ser implementado pelas Hamiltonianas:

$$
H=H_{0}+\alpha_{1} L_{1}^{2}+\alpha_{2} L_{2}^{2}+\beta_{2} L_{2, z}^{2}+\gamma_{2}\left(L_{2}^{2}-2\right)\left(L_{2}^{2}-6\right)\left(L_{2}^{2}-\frac{35}{4}\right) L_{2, z},
$$

para a cadeia de $G_{2}$ e

$$
H=H_{0}+\lambda C_{2}(s p(4))+\alpha_{1} L_{1}^{2}+\alpha_{2} L_{2}^{2}+\alpha_{3} L_{3}^{2}+\beta_{2} L_{2, z}^{2}+\gamma_{3}\left(L_{1}^{2}+L_{2}^{2}\right)\left(L_{3}^{2}-2\right) L_{3,2},
$$

para a cadeia de $\mathrm{sp}(6)$.

\subsection{Análise das Cadeias do $\mathrm{B}_{6}$ e do $\mathrm{D}_{7}$ na Fase 2}

Apresentaremos nesta seção a análise na fase 2 de todas as cadeias de $B_{6}$ e de $D_{7}$, sobreviventes à fase 1 , ou seja, submeteremos essas cadeias a uma segunda análise mais completa, em que as representações do su(2) são quebradas para o(2) e so(2), através dos operadores $L_{z}^{2}$ e $L_{z}$, respectivamente. Aplicamos as mesmas estratégias gerais que já foram utilisadas antes:

\begin{tabular}{|c|c|c|c|c|}
\hline $\mathrm{B}_{6}$ & $D_{6}$ & $\mathrm{~A}_{1} \oplus \mathrm{C}_{3}$ & $\mathrm{~A}_{1} \oplus \mathrm{A}_{1}$ & $\mathrm{~d}$ \\
\hline \multirow[t]{6}{*}{$(000001 ; 64)$} & \multirow[t]{3}{*}{$(000010 ; 32)$} & \multirow[t]{2}{*}{$(0,001 ; 14)$} & $(0,9)$ & 10 \\
\hline & & & $(0,3)$ & 4 \\
\hline & & $(2,100 ; 18)$ & $(2,5)$ & 18 \\
\hline & \multirow[t]{3}{*}{$(000001 ; 32)$} & \multirow[t]{2}{*}{$(1,010 ; 28)$} & $(1,8)$ & 18 \\
\hline & & & $(1,4)$ & 10 \\
\hline & & $(3,000 ; 4)$ & $(3,0)$ & 4 \\
\hline
\end{tabular}

Começamos com a cadeia 5 , listada na tabela 14 , que é a primeira cadeia da álgebra $\mathrm{B}_{6}$, classificada como sobrevivente à fase 1 no capítulo 4 .

Tabela 14: Cadeia 5: $B_{6} \supset D_{6} \supset A_{1} \oplus C_{3} \supset A_{1} \oplus A_{1}$. 
Inicialmente, observamos que a segunda subálgebra $A_{1}$ tem que ser quebrada, para eliminar as representações de dimensão 9,10 e 18, pois não existem códons com degenerescência 5 ou 9 ou 10 ou 18. Mas se quebramos em $L_{2,2}^{2}$, que é a quebra mais suave, geramos 19 multipletos com $d_{3}=18$ (veja a tabela $14 a$ ), o que elimina qualquer possibilidade de prosseguir.

\begin{tabular}{|c|c|c|c|}
\hline$A_{1} \oplus A_{1}$ & d & $L_{2, z}^{2}$ & d \\
\hline \multirow[t]{5}{*}{$(0,9)$} & \multirow[t]{5}{*}{10} & $(0, \pm 9)$ & 2 \\
\hline & & $(0, \pm 7)$ & 2 \\
\hline & & $(0, \pm 5)$ & 2 \\
\hline & & $(0, \pm 3)$ & 2 \\
\hline & & $(0, \pm 1)$ & 2 \\
\hline \multirow[t]{2}{*}{$(0,3)$} & \multirow[t]{2}{*}{4} & $(0, \pm 3)$ & 2 \\
\hline & & $(0, \pm 1)$ & 2 \\
\hline \multirow[t]{3}{*}{$(2,5)$} & \multirow[t]{3}{*}{18} & $(2, \pm 5)$ & 6 \\
\hline & & $(2, \pm 3)$ & 6 \\
\hline & & $(2, \pm 1)$ & 6 \\
\hline \multirow[t]{5}{*}{$(1,8)$} & \multirow[t]{5}{*}{18} & $(1, \pm 8)$ & 4 \\
\hline & & $(1, \pm 6)$ & 4 \\
\hline & & $(1, \pm 4)$ & 4 \\
\hline & & $(1, \pm 2)$ & 4 \\
\hline & & $(1,0)$ & 2 \\
\hline \multirow[t]{3}{*}{$(1,4)$} & \multirow[t]{3}{*}{10} & $(1, \pm 4)$ & 4 \\
\hline & & $(1, \pm 2)$ & 4 \\
\hline & & $(1,0)$ & 2 \\
\hline$(3,0)$ & 4 & $(3,0)$ & 4 \\
\hline \multicolumn{2}{|c|}{6 subespaços } & \multicolumn{2}{|c|}{19 subespaços } \\
\hline \multicolumn{2}{|c|}{$d_{3}=36$} & \multicolumn{2}{|c|}{$\mathrm{d}_{3}=18$} \\
\hline
\end{tabular}

Tabela 14a: Quebra da cadeia 5, em $L_{2, z}^{2}$ (não-sobrevivente).

Analisaremos agora a próxima cadeia de $\mathrm{B}_{6}$ sobrevivente à fase $\mathrm{I}$, que é a cadeia 6 listada na tabela 15.

\begin{tabular}{|c|c|c|c|}
\hline $\mathrm{B}_{6}$ & $\mathrm{D}_{6}$ & $\mathrm{~A}_{1} \oplus \mathrm{A}_{1} \oplus \mathrm{A}_{1}$ & $\mathrm{~d}$ \\
\hline$(000001 ; 64)$ & $(000001 ; 32)$ & $(4,1,0)$ & 10 \\
\cline { 3 - 4 } & & $(2,1,2)$ & 18 \\
\cline { 3 - 4 } & & $(0,3,0)$ & 4 \\
\hline & $(000010 ; 32)$ & $(4,0,1)$ & 10 \\
\cline { 3 - 4 } & & $(2,2,1)$ & 18 \\
\cline { 3 - 4 } & & $(0,0,3)$ & 4 \\
\hline
\end{tabular}

Tabela 15: Cadeia 6: $B_{6} \supset D_{6} \supset A_{1} \oplus A_{1} \oplus A_{1}$. 
Inicialmente, observamos que a primeira subálgebra $A_{1}$ tem que ser quebrada, para eliminar as representações de dimensão 10 , pois não existem códons com degenerescência 5 ou 10. Observamos também que existe simetria sob a troca do segundo e terceiro $A_{1}$. Portanto, quebramos primeiro em $L_{1,2}^{2}$, gerando 12 multipletos com $d_{3}=36$ (veja as tabelas 15a e 15b), e devemos continuar o processo. Quebrando depois em $L_{1,2}$, obtemos 18 multipletos com $\mathrm{d}_{3}=36$ (veja a tabela 15a), mas não há um número suficiente de multipletos de dimensão $\geq 3$ (apenas 6 sextupletos e 2 quadrupletos) para acomodar 3 sextupletos, 5 quadrupletos e 2 tripletos. Por outro lado, quebrando depois em $L_{2, z}^{2}$, por exemplo, obtemos 14 multipletos com $\mathrm{d}_{3}=18$ (veja a tabela 15b).. Como estas são as quebras mais suaves, está eliminada qualquer possibilidade de prosseguir.

\begin{tabular}{|c|c|c|c|c|c|}
\hline $\mathrm{A}_{1} \oplus \mathrm{A}_{1} \oplus \mathrm{A}_{1}$ & $\mathrm{~d}$ & $L_{1 . z}^{2}$ & $\mathrm{~d}$ & $L_{1, z}$ & d \\
\hline \multirow[t]{5}{*}{$\overline{(4,1,0)}$} & \multirow[t]{5}{*}{10} & \multirow[t]{2}{*}{$( \pm 4,1,0)$} & \multirow[t]{2}{*}{4} & $(+4,1,0)$ & 2 \\
\hline & & & & $(-4,1,0)$ & 2 \\
\hline & & \multirow[t]{2}{*}{$( \pm 2,1,0)$} & \multirow[t]{2}{*}{4} & $(+2,1,0)$ & 2 \\
\hline & & & & $(-2,1,0)$ & 2 \\
\hline & & $(0,1,0)$ & 2 & $(0,1,0)$ & 2 \\
\hline \multirow[t]{3}{*}{$(2,1,2)$} & \multirow[t]{3}{*}{18} & \multirow[t]{2}{*}{$( \pm 2,1,2)$} & \multirow[t]{2}{*}{12} & $(+2,1,2)$ & 6 \\
\hline & & & & $(-2,1,2)$ & 6 \\
\hline & & $(0,1,2)$ & 6 & $(0,1,2)$ & 6 \\
\hline$(0,3,0)$ & 4 & $(0,3,0)$ & 4 & $(0,3,0)$ & 4 \\
\hline \multirow[t]{5}{*}{$(4,0,1)$} & \multirow[t]{5}{*}{10} & \multirow[t]{2}{*}{$( \pm 4,0,1)$} & \multirow[t]{2}{*}{4} & $(+4,0,1)$ & 2 \\
\hline & & & & $(-4,0,1)$ & 2 \\
\hline & & \multirow[t]{2}{*}{$( \pm 2,0,1)$} & \multirow[t]{2}{*}{4} & $(+2,0,1)$ & 2 \\
\hline & & & & $(-2,0,1)$ & 2 \\
\hline & & $(0,0,1)$ & 2 & $(0,0,1)$ & 2 \\
\hline \multirow[t]{3}{*}{$(2,2,1)$} & \multirow[t]{3}{*}{18} & \multirow[t]{2}{*}{$( \pm 2,2,1)$} & \multirow[t]{2}{*}{12} & $(+2,2,1)$ & $\frac{5}{6}$ \\
\hline & & & & $(-2,2,1)$ & 6 \\
\hline & & $(0,2,1)$ & 6 & $(0,2,1)$ & 6 \\
\hline$(0,0,3)$ & 4 & $(0,0,3)$ & 4 & $(0,0,3)$ & 4 \\
\hline \multicolumn{2}{|l|}{6 subespaços } & \multicolumn{2}{|c|}{12 subespaços } & \multicolumn{2}{|c|}{18 subespaços } \\
\hline \multicolumn{2}{|l|}{$d_{3}=36$} & \multicolumn{2}{|c|}{$\mathrm{d}_{3}=36$} & \multicolumn{2}{|c|}{$d_{3}=36$} \\
\hline
\end{tabular}

Tabela 15a: Quebra da cadeia 6, primeiro em $L_{1, z}^{2}$ e depois em $L_{1,2}$ (não-sobrevivente). 


\begin{tabular}{|c|c|c|c|c|c|}
\hline $\mathrm{A}_{1} \oplus \mathrm{A}_{1} \oplus \mathrm{A}_{1}$ & d & $L_{1,2}^{2}$ & $\mathrm{~d}$ & $\left(L_{1,2}^{2}, L_{2, z}^{2}\right)$ & $\mathrm{d}$ \\
\hline \multirow[t]{3}{*}{$(4,1,0)$} & \multirow[t]{3}{*}{10} & $( \pm 4,1,0)$ & 4 & $( \pm 4, \pm 1,0)$ & 4 \\
\hline & & $( \pm 2,1,0)$ & 4 & $( \pm 2, \pm 1,0)$ & 4 \\
\hline & & $(0,1,0)$ & 2 & $(0, \pm 1,0)$ & 2 \\
\hline \multirow[t]{2}{*}{$(2,1,2)$} & \multirow[t]{2}{*}{18} & $( \pm 2,1,2)$ & 12 & $( \pm 2, \pm 1,2)$ & 12 \\
\hline & & $(0,1,2)$ & 6 & $(0, \pm 1,2)$ & 6 \\
\hline \multirow[t]{2}{*}{$(0,3,0)$} & \multirow[t]{2}{*}{4} & \multirow[t]{2}{*}{$(0,3,0)$} & \multirow[t]{2}{*}{4} & $(0, \pm 3,0)$ & 2 \\
\hline & & & & $(0, \pm 1,0)$ & 2 \\
\hline \multirow[t]{3}{*}{$(4,0,1)$} & \multirow[t]{3}{*}{10} & $( \pm 4,0,1)$ & 4 & $( \pm 4,0,1)$ & 4 \\
\hline & & $( \pm 2,0,1)$ & 4 & $( \pm 2,0,1)$ & 4 \\
\hline & & $(0,0,1)$ & 2 & $(0,0,1)$ & 2 \\
\hline \multirow[t]{4}{*}{$(2,2,1)$} & \multirow[t]{4}{*}{18} & \multirow[t]{2}{*}{$( \pm 2,2,1)$} & \multirow[t]{2}{*}{12} & $( \pm 2, \pm 2,1)$ & 8 \\
\hline & & & & $( \pm 2,0,1)$ & 4 \\
\hline & & \multirow[t]{2}{*}{$(0,2,1)$} & \multirow[t]{2}{*}{6} & $(0, \pm 2,1)$ & 4 \\
\hline & & & & $(0,0,1)$ & 2 \\
\hline$(0,0,3)$ & 4 & $(0,0,3)$ & 4 & $(0,0,3)$ & 4 \\
\hline \multicolumn{2}{|l|}{6 subespacos } & \multicolumn{2}{|c|}{12 subespaços } & \multicolumn{2}{|c|}{14 subespaços } \\
\hline \multicolumn{2}{|l|}{$d_{3}=36$} & \multicolumn{2}{|c|}{$\mathrm{d}_{3}=36$} & \multicolumn{2}{|c|}{$\mathrm{d}_{3}=18$} \\
\hline
\end{tabular}

Tabela 15b: Quebra da cadeia 6, primeiro em $L_{1,2}^{2}$ e depois em $L_{2,2}^{2}$ (não-sobrevivente).

Analisaremos agora as cadeias de $D_{7}$ sobreviventes à fase 1 , começando com a cadeia 7 listada na tabela 16.

\begin{tabular}{|c|c|c|c|c|c|c|}
\hline $\mathrm{D}_{7}$ & $\mathrm{~B}_{3} \oplus \mathrm{B}_{3}$ & $\mathrm{~B}_{3} \oplus \mathrm{G}_{2}$ & $\mathrm{G}_{2} \oplus \mathrm{G}_{2}$ & $\mathrm{G}_{2} \oplus \mathrm{A}_{1}$ & $\mathrm{~A}_{1} \oplus \mathrm{A}_{1} \oplus \mathrm{A}_{1}$ & d \\
\hline \multirow[t]{6}{*}{$(0000001 ; 64)$} & \multirow[t]{6}{*}{$(001,001 ; 64)$} & \multirow[t]{3}{*}{$(001,01 ; 56)$} & \multirow[t]{2}{*}{$(01,01 ; 49)$} & \multirow[t]{2}{*}{$(01,6 ; 49)$} & $(1,1,6)$ & 28 \\
\hline & & & & & $(0,2,6)$ & 21 \\
\hline & & & $(00,01 ; 7)$ & $(00,6 ; 7)$ & $(0,0,6)$ & 7 \\
\hline & & \multirow[t]{3}{*}{$(001,00 ; 8)$} & \multirow[t]{2}{*}{$(01,00 ; 7)$} & \multirow[t]{2}{*}{$(01,0 ; 7)$} & $(1,1,0)$ & 4 \\
\hline & & & & & $(0,2,0)$ & 3 \\
\hline & & & $(00,00 ; 1)$ & $(00,0 ; 1)$ & $(0,0,0)$ & 1 \\
\hline
\end{tabular}

Tabela 16: Cadeia 7: $D_{7} \supset B_{3} \oplus B_{3} \supset B_{3} \oplus G_{2} \supset G_{2} \oplus G_{2} \supset G_{2} \oplus A_{1} \supset A_{1} \oplus A_{1} \oplus A_{1}$.

Analisando esta cadeia segundo as estratégias estabelecidas para a análise da fase 2, verificamos que o último $\mathrm{A}_{\mathrm{I}}$ tem que ser quebrado, não há quebras em $L_{1,2}^{2}$, o segundo $\mathrm{A}_{1}$ não pode ser quebrado em $L_{2, z}$ e $L_{2.2}^{2}$, pois o primeiro caso, produz um número excessivo de multipletos de dimensão 1 e no segundo caso o $d_{3}$ cai para zero, e o último $A_{1}$ não pode ser quebrado em $L_{3, z}$, pois isto produz um número excessivo de multipletos de dimensão impar (8 
singletos, no total), então as únicas quebras possíveis são em $L_{3, z}^{2}$ e $\left(L_{1, z}, L_{3, z}^{2}\right)$, as quais podem serem vistas nas tabelas $16 \mathrm{a}$ e $16 \mathrm{~b}$.

\begin{tabular}{|c|c|c|c|}
\hline$A_{1} \oplus A_{1} \oplus A_{1}$ & d & $L_{3 . z}^{2}$ & $\mathrm{~d}$ \\
\hline \multirow[t]{4}{*}{$(1,1,6)$} & \multirow[t]{4}{*}{28} & $(1,1, \pm 6)$ & 8 \\
\hline & & $(1,1, \pm 4)$ & 8 \\
\hline & & $(1,1, \pm 2)$ & 8 \\
\hline & & $(1,1,0)$ & 4 \\
\hline \multirow[t]{4}{*}{$(0,2,6)$} & \multirow[t]{4}{*}{21} & $(0,2, \pm 6)$ & $\overline{6}$ \\
\hline & & $(0,2, \pm 4)$ & 6 \\
\hline & & $(0,2, \pm 2)$ & 6 \\
\hline & & $(0,2,0)$ & 3 \\
\hline \multirow[t]{4}{*}{$(0,0,6)$} & \multirow[t]{4}{*}{7} & $(0,0, \pm 6)$ & 2 \\
\hline & & $(0,0, \pm 4)$ & 2 \\
\hline & & $(0,0, \pm 2)$ & 2 \\
\hline & & $(0,0,0)$ & 1 \\
\hline$(1,1,0)$ & 4 & $(1,1,0)$ & 4 \\
\hline$(0,2,0)$ & 3 & $(0,2,0)$ & 3 \\
\hline$(0,0,0)$ & 1 & $(0,0,0)$ & 1 \\
\hline \multicolumn{2}{|c|}{6 subespaços } & \multicolumn{2}{|c|}{15 subespaços } \\
\hline \multicolumn{2}{|c|}{$\mathrm{d}_{3}=24$} & \multicolumn{2}{|c|}{$\mathrm{d}_{3}=24$} \\
\hline
\end{tabular}

Tabela 16a: Quebra da cadeia 7, em $L_{3, z}^{2}$ (não-sobrevivente). 


\begin{tabular}{|c|c|c|c|c|c|}
\hline $\mathrm{A}_{1} \oplus \mathrm{A}_{1} \oplus \mathrm{A}_{1}$ & d & $L_{1,2}$ & $\bar{d}$ & $L_{3.2}^{2}$ & d \\
\hline \multirow[t]{8}{*}{$(1,1,6)$} & \multirow[t]{8}{*}{28} & \multirow[t]{4}{*}{$(+1,1,6)$} & \multirow[t]{4}{*}{14} & $(+1,1, \pm 6)$ & 4 \\
\hline & & & & $(+1,1, \pm 4)$ & 4 \\
\hline & & & & $(+1,1, \pm 2)$ & 4 \\
\hline & & & & $(+1,1,0)$ & 2 \\
\hline & & \multirow[t]{4}{*}{$(-1,1,6)$} & \multirow[t]{4}{*}{14} & $(-1,1, \pm 6)$ & 4 \\
\hline & & & & $(-1,1, \pm 4)$ & 4 \\
\hline & & & & $(-1,1, \pm 2)$ & 4 \\
\hline & & & & $(-1,1,0)$ & 2 \\
\hline \multirow[t]{4}{*}{$(0,2,6)$} & \multirow[t]{4}{*}{21} & \multirow[t]{4}{*}{$(0,2,6)$} & \multirow[t]{4}{*}{21} & $(0,2, \pm 6)$ & 6 \\
\hline & & & & $(0,2, \pm 4)$ & 6 \\
\hline & & & & $(0,2, \pm 2)$ & 6 \\
\hline & & & & $(0,2,0)$ & 3 \\
\hline \multirow[t]{4}{*}{$(0,0,6)$} & \multirow[t]{4}{*}{7} & \multirow[t]{4}{*}{$(0,0,6)$} & \multirow[t]{4}{*}{7} & $(0,0, \pm 6)$ & 2 \\
\hline & & & & $(0,0, \pm 4)$ & 2 \\
\hline & & & & $(0,0, \pm 2)$ & 2 \\
\hline & & & & $(0,0,0)$ & 1 \\
\hline \multirow[t]{2}{*}{$(1,1,0)$} & \multirow[t]{2}{*}{4} & $(+1,1,0)$ & 2 & $(+1,1,0)$ & 2 \\
\hline & & $(-1,1,0)$ & 2 & $(-1,1,0)$ & 2 \\
\hline$(0,2,0)$ & 3 & $(0,2,0)$ & 3 & $(0,2,0)$ & 3 \\
\hline$(0,0,0)$ & 1 & $(0,0,0)$ & 1 & $(0,0,0)$ & 1 \\
\hline \multicolumn{2}{|c|}{6 subespac } & \multicolumn{2}{|c|}{8 subespaços } & \multicolumn{2}{|c|}{20 subespaços } \\
\hline \multicolumn{2}{|c|}{$\mathrm{d}_{3}$} & \multicolumn{2}{|c|}{$\mathrm{d}_{3}$} & \multicolumn{2}{|c|}{$\mathrm{d}_{3}$} \\
\hline
\end{tabular}

Tabela 16b: Quebra da cadeia 7, primeiro em $L_{1, z}$ e depois em $L_{3, z}^{2}$ (não-sobrevivente).

Listaremos agora na tabela 17 outra cadeia sobrevivente à fase 1 , a cadeia 8 , a qual será submetida à fase 2 .

\begin{tabular}{|c|c|c|c|c|c|c|}
\hline $\mathrm{D}_{7}$ & $\mathrm{~A}_{3} \oplus \mathrm{D}_{4}$ & $\mathrm{~A}_{3} \oplus \mathrm{A}_{1} \oplus \mathrm{C}_{2}$ & $\mathrm{~A}_{3} \oplus \mathrm{A}_{1} \oplus \mathrm{A}_{1}$ & $\mathrm{~A}_{2} \oplus \mathrm{A}_{1} \oplus \mathrm{A}_{1}$ & $\mathrm{~A}_{1} \oplus \mathrm{A}_{1} \oplus \mathrm{A}_{1}$ & $\mathrm{~d}$ \\
\hline \multirow{3}{*}{$(0000001 ; 64)$} & $(001,0010 ; 32)$ & $(001,0,01 ; 20)$ & $(001,0,4 ; 20)$ & $(01,0,4 ; 15)$ & $(2,0,4)$ & 15 \\
\cline { 5 - 7 } & & & & $(00,0,4 ; 5)$ & $(0,0,4)$ & 5 \\
\cline { 5 - 7 } & & $(001,2,00 ; 12)$ & $(001,2-0 ; 12)$ & $(01,2,0 ; 9)$ & $(2,2,0)$ & 9 \\
\cline { 5 - 7 } & & & $(00,2,0 ; 3)$ & $(0,2,0)$ & 3 \\
\cline { 5 - 8 } & $(100,0001 ; 32)$ & $(100,1,10 ; 32)$ & $(100,1,3 ; 32)$ & $(10,1,3 ; 24)$ & $(2,1,3)$ & 24 \\
\cline { 5 - 8 } & & & $(00,1,3 ; 8)$ & $(0,1,3)$ & 8 \\
\hline
\end{tabular}

Tabela 17: Cadeia 8: $\mathrm{D}_{7} \supset \mathrm{A}_{3} \oplus \mathrm{D}_{4} \supset \mathrm{A}_{3} \oplus \mathrm{A}_{1} \oplus \mathrm{C}_{2} \supset \mathrm{A}_{3} \oplus \mathrm{A}_{1} \oplus \mathrm{A}_{1} \supset \mathrm{A}_{2} \oplus \mathrm{A}_{1} \oplus \mathrm{A}_{1}$ $\supset A_{1} \oplus A_{1} \oplus A_{1}$.

Observando a cadeia 8 verificamos que a quebra da terceira álgebra $A_{1}$ é inevitável. A existencia de representações de dimensð̃es 5 e 15 só podem e devem serem eliminadas através dessa quebra, pois não existem códons com degenerescências multiplos de 5 e não podemos quebrar diretamente $o$ terceiro $A_{i}$, pois nesta quebra geramos singletos em excesso. Nesta 
cadeia o primeiro ou o segundo $A_{1}$ não pode ser quebrado, pois se quebrarmos o primeiro $A_{1}, 0$ $\mathrm{d}_{3}$ cai para, no máximo, 12, daí o segundo $\mathrm{A}_{1}$ tem que ser quebrado, já que o primeiro não é, para que o multipleto de dimensão 9 seja eliminado. Portanto as quebras possíveis são em $\left(L_{2, z}^{2}, L_{3, z}^{2}\right),\left(L_{2, z}^{2}, L_{3, z}\right)$ e $L_{2, z}$, as quais podem serem vistas nas tabelas $17 \mathrm{a}$ e $17 \mathrm{~b}$.

\begin{tabular}{|c|c|c|c|c|c|c|c|}
\hline $\mathrm{A}_{1} \oplus \mathrm{A}_{1} \oplus \mathrm{A}_{1}$ & $\bar{d}$ & $L_{2, z}^{2}$ & $\bar{d}$ & $\left(L_{2, z}^{2}, L_{3, z}^{2}\right)$ & $\mathrm{d}$ & $\left(L_{2, z}^{2}, L_{3, z}\right)$ & d \\
\hline \multirow[t]{5}{*}{$(2,0,4)$} & \multirow[t]{5}{*}{15} & \multirow[t]{5}{*}{$(2,0,4)$} & \multirow[t]{5}{*}{15} & \multirow[t]{2}{*}{$(2,0, \pm 4)$} & \multirow[t]{2}{*}{6} & $(2,0,+4)$ & 3 \\
\hline & & & & & & $(2,0,-4)$ & 3 \\
\hline & & & & \multirow[t]{2}{*}{$(2,0, \pm 2)$} & \multirow[t]{2}{*}{6} & $(2,0,+2)$ & 3 \\
\hline & & & & & & $(2,0,-2)$ & 3 \\
\hline & & & & $(2,0,0)$ & 3 & $(2,0,0)$ & 3 \\
\hline \multirow[t]{5}{*}{$(0,0,4)$} & \multirow[t]{5}{*}{5} & \multirow[t]{5}{*}{$(0,0,4)$} & \multirow[t]{5}{*}{5} & \multirow[t]{2}{*}{$(0,0, \pm 4)$} & \multirow[t]{2}{*}{2} & $(0,0,+4)$ & 1 \\
\hline & & & & & & $(0,0,-4)$ & 1 \\
\hline & & & & \multirow[t]{2}{*}{$(0,0, \pm 2)$} & \multirow[t]{2}{*}{2} & $(0,0,+2)$ & 1 \\
\hline & & & & & & $(0,0,-2)$ & 1 \\
\hline & & & & $(0,0,0)$ & 1 & $(0,0,0)$ & 1 \\
\hline \multirow[t]{2}{*}{$(2,2,0)$} & \multirow[t]{2}{*}{9} & $(2, \pm 2,0)$ & 6 & $(2, \pm 2,0)$ & 6 & $(2, \pm 2,0)$ & 6 \\
\hline & & $(2,0,0)$ & 3 & $(2,0,0)$ & 3 & $(2,0,0)$ & 3 \\
\hline \multirow[t]{2}{*}{$(0,2,0)$} & \multirow[t]{2}{*}{3} & $(0, \pm 2,0)$ & 2 & $(0, \pm 2,0)$ & 2 & $(0, \pm 2,0)$ & 2 \\
\hline & & $(0,0,0)$ & 1 & $(0,0,0)$ & 1 & $(0,0,0)$ & 1 \\
\hline \multirow[t]{4}{*}{$(2,1,3)$} & \multirow[t]{4}{*}{24} & \multirow[t]{4}{*}{$(2, \pm 1,3)$} & \multirow[t]{4}{*}{24} & \multirow[t]{2}{*}{$(2, \pm 1, \pm 3)$} & \multirow[t]{2}{*}{8} & $(2, \pm 1,+3)$ & 4 \\
\hline & & & & & & $(2, \pm 1,-3)$ & 4 \\
\hline & & & & \multirow[t]{2}{*}{$(2, \pm 1, \pm 1)$} & \multirow[t]{2}{*}{8} & $(2, \pm 1,+1)$ & 4 \\
\hline & & & & & & $(2, \pm 1,-1)$ & 4 \\
\hline \multirow[t]{4}{*}{$(0,1,3)$} & \multirow[t]{4}{*}{8} & \multirow[t]{4}{*}{$(0, \pm 1,3)$} & \multirow[t]{4}{*}{8} & \multirow[t]{2}{*}{$(0, \pm 1, \pm 3)$} & 4 & $(0, \pm 1,+3)$ & 2 \\
\hline & & & & & & $(0, \pm 1,-3)$ & 2 \\
\hline & & & & $(0, \pm 1, \pm 1)$ & 4 & $(0, \pm 1,+1)$ & 2 \\
\hline & & & & & & $(0, \pm 1,-1)$ & 2 \\
\hline 6 subespac & & 8 subesp & & 14 subesp & & 22 subess & \\
\hline $\mathrm{d}_{3}=36$ & & $\mathrm{~d}_{3}=4$ & & $\mathrm{~d}_{3}=2$ & & $d_{3}=2$ & \\
\hline
\end{tabular}

Tabela 17a: Quebra da cadeia 8 primeiro em $L_{2, z}^{2}$ e depois em $L_{3, z}^{2}$ e $L_{3, z}$ (não-sobrevivente). 


\begin{tabular}{|c|c|c|c|}
\hline $\mathrm{A}_{1} \oplus \mathrm{A}_{1} \oplus \mathrm{A}_{1}$ & d & $L_{2.2}$ & $\mathrm{~d}$ \\
\hline$(2,0,4)$ & 15 & $(2,0,4)$ & 15 \\
\hline$(0,0,4)$ & 5 & $(0,0,4)$ & 5 \\
\hline \multirow[t]{3}{*}{$(2,2,0)$} & \multirow[t]{3}{*}{9} & $(2,+2,0)$ & 3 \\
\hline & & $(2,-2,0)$ & 3 \\
\hline & & $(2,0,0)$ & 3 \\
\hline \multirow[t]{3}{*}{$(0,2,0)$} & \multirow[t]{3}{*}{3} & $(0,+2,0)$ & 1 \\
\hline & & $(0,-2,0)$ & 1 \\
\hline & & $(0,0,0)$ & 1 \\
\hline \multirow[t]{2}{*}{$(2,1,3)$} & \multirow[t]{2}{*}{24} & $(2,+1,3)$ & 12 \\
\hline & & $(2,-1,3)$ & 12 \\
\hline \multirow[t]{2}{*}{$(0,1,3)$} & \multirow[t]{2}{*}{8} & $(0,+1,3)$ & 4 \\
\hline & & $(0,-1,3)$ & 4 \\
\hline \multicolumn{2}{|c|}{6 subespaços } & \multicolumn{2}{|c|}{12 subespaços } \\
\hline \multicolumn{2}{|c|}{$\mathrm{d}_{3}=48$} & \multicolumn{2}{|c|}{$\mathrm{d}_{3}=48$} \\
\hline
\end{tabular}

Tabela 17b: Quebra da cadeia $8 \mathrm{em} L_{2, \mathrm{z}}$ (não-sobrevivente).

A próxima cadeia sobrevivente a fase 1 submetida à fase 2 é a cadeia 9 listada na tabela 18 .

\begin{tabular}{|c|c|c|c|c|}
\hline $\mathrm{D}_{7}$ & $\mathrm{~A}_{1} \oplus \mathrm{A}_{1} \oplus \mathrm{D}_{5}$ & $\mathrm{~A}_{1} \oplus \mathrm{A}_{1} \oplus \mathrm{C}_{2}$ & $\mathrm{~A}_{1} \oplus \mathrm{A}_{1} \oplus \mathrm{A}_{1} \oplus \mathrm{A}_{1}$ & $\mathrm{~d}$ \\
\hline$(0000001 ; 64)$ & $(0,1,00010 ; 32)$ & $(0,1,11 ; 32)$ & $(0,1,2,1)$ & 12 \\
\cline { 4 - 5 } & & & $(0,1,1,2)$ & 12 \\
\cline { 4 - 5 } & & & $(0,1,1,0)$ & 4 \\
\cline { 4 - 5 } & $(1,0,00001 ; 32)$ & \multirow{2}{*}{$(1,0,11 ; 32)$} & $(1,0,1,0,1)$ & 4 \\
\cline { 4 - 5 } & & & $(1,0,1,2)$ & 12 \\
\cline { 4 - 5 } & & & $(1,0,1,0)$ & 4 \\
\cline { 4 - 5 } & & & $(1,0,0,1)$ & 4 \\
\hline
\end{tabular}

Tabela 18: Cadeia $9: D_{7} \supset A_{1} \oplus A_{1} \oplus D_{5} \supset A_{1} \oplus A_{1} \oplus C_{2} \supset A_{1} \oplus A_{1} \oplus A_{1} \oplus A_{1}$

Aplicando as estratégias, para a análise da segunda fase, na cadeia 9 verificamos que não há quebras com $L_{1,2}^{2}$ e $L_{2,2}^{2}$. Existe simetria sob a troca do primeiro e segundo $A_{1}$ e do terceiro e quarto $A_{1}$. O terceiro ou quarto $A_{1}$ deve ser quebrado, digamos o terceiro, pois senão, todos os multipletos teriam multiplicidade par, e não teriamos tripletos ou singletos. Portanto as quebras possíveis são em $L_{3, z}^{2}, L_{3, z},\left(L_{3, z}^{2}, L_{1, z}\right)$ e $\left(L_{3, z}, L_{1, z}\right)$, que podem ser em vistas nas tabelas $18 \mathrm{a}$ e $18 \mathrm{~b}$. 


\begin{tabular}{|c|c|c|c|c|c|}
\hline$A_{1} \oplus A_{1} \oplus A_{1} \oplus A_{1}$ & $\mathrm{~d}$ & $L_{3 . z}^{2}$ & d & $\left(L_{3, z}^{2}, L_{1, z}\right)$ & d \\
\hline \multirow[t]{2}{*}{$(0,1,2,1)$} & \multirow[t]{2}{*}{12} & $(0,1, \pm 2,1)$ & 8 & $(0,1, \pm 2,1)$ & 8 \\
\hline & & $(0,1,0,1)$ & 4 & $(0,1,0,1)$ & 4 \\
\hline$(0,1,1,2)$ & 12 & $(0,1, \pm 1,2)$ & 12 & $(0,1, \pm 1,2)$ & 12 \\
\hline$(0,1,1,0)$ & 4 & $(0,1, \pm 1,0)$ & 4 & $(0,1, \pm 1,0)$ & 4 \\
\hline$(0,1,0,1)$ & 4 & $(0,1,0,1)$ & 4 & $(0,1,0,1)$ & 4 \\
\hline \multirow[t]{4}{*}{$(1,0,2,1)$} & \multirow[t]{4}{*}{12} & \multirow[t]{2}{*}{$(1,0, \pm 2,1)$} & \multirow[t]{2}{*}{8} & $(+1,0, \pm 2,1)$ & 4 \\
\hline & & & & $(-1,0, \pm 2,1)$ & 4 \\
\hline & & \multirow[t]{2}{*}{$(1,0,0,1)$} & \multirow[t]{2}{*}{4} & $(+1,0,0,1)$ & 2 \\
\hline & & & & $(-1,0,0,1)$ & 2 \\
\hline \multirow[t]{2}{*}{$(1,0,1,2)$} & \multirow[t]{2}{*}{12} & \multirow[t]{2}{*}{$(1,0, \pm 1,2)$} & \multirow[t]{2}{*}{12} & $(+1,0, \pm 1,2)$ & 6 \\
\hline & & & & $(-1,0, \pm 1,2)$ & 6 \\
\hline \multirow[t]{2}{*}{$(1,0,1,0)$} & \multirow[t]{2}{*}{4} & \multirow[t]{2}{*}{$(1,0, \pm 1,0)$} & \multirow[t]{2}{*}{4} & $(+1,0, \pm 1,0)$ & 2 \\
\hline & & & & $(-1,0, \pm 1,0)$ & 2 \\
\hline \multirow[t]{2}{*}{$(1,0,0,1)$} & \multirow[t]{2}{*}{4} & \multirow[t]{2}{*}{$(1,0,0,1)$} & \multirow[t]{2}{*}{4} & $(+1,0,0,1)$ & 2 \\
\hline & & & & $(-1,0,0,1)$ & 2 \\
\hline \multicolumn{2}{|c|}{8 subespaços } & \multicolumn{2}{|c|}{10 subespaços } & \multicolumn{2}{|c|}{15 subespaços } \\
\hline \multicolumn{2}{|c|}{$\mathrm{d}_{3}=48$} & \multicolumn{2}{|c|}{$\mathrm{d}_{3}=24$} & \multicolumn{2}{|c|}{$\mathrm{d}_{3}=24$} \\
\hline
\end{tabular}

Tabela 18a: Quebra da cadeia 9 em $L_{3, z}^{2}$ e depois em $L_{1, z}$ (não-sobrevivente).

\begin{tabular}{|c|c|c|c|c|c|}
\hline $\mathrm{A}_{1} \oplus \mathrm{A}_{1} \oplus \mathrm{A}_{1} \oplus \mathrm{A}_{1}$ & d & $L_{3,2}$ & d & $\left(L_{3 ., z}, L_{1,2}\right)$ & $\mathrm{d}$ \\
\hline \multirow[t]{3}{*}{$(0,1,2,1)$} & \multirow[t]{3}{*}{12} & $(0,1,+2,1)$ & 4 & $(0,1,+2,1)$ & 4 \\
\hline & & $(0,1,-2,1)$ & 4 & $(0,1,-2,1)$ & 4 \\
\hline & & $(0,1,0,1)$ & 4 & $(0,1,0,1)$ & 4 \\
\hline \multirow[t]{2}{*}{$(0,1,1,2)$} & \multirow[t]{2}{*}{12} & $(0,1,+1,2)$ & 6 & $(0,1,+1,2)$ & 6 \\
\hline & & $(0,1,-1,2)$ & 6 & $(0,1,-1,2)$ & 6 \\
\hline \multirow[t]{2}{*}{$(0,1,1,0)$} & \multirow[t]{2}{*}{4} & $(0,1,+1,0)$ & 2 & $(0,1,+1,0)$ & 2 \\
\hline & & $(0,1,-1,0)$ & 2 & $(0,1,-1,0)$ & 2 \\
\hline$(0,1,0,1)$ & 4 & $(0,1,0,1)$ & 4 & $(0,1,0,1)$ & 4 \\
\hline \multirow[t]{6}{*}{$(1,0,2,1)$} & \multirow[t]{6}{*}{12} & \multirow[t]{2}{*}{$(1,0,+2,1)$} & \multirow[t]{2}{*}{4} & $(+1,0,+2,1)$ & 2 \\
\hline & & & & $(-1,0,+2,1)$ & 2 \\
\hline & & \multirow[t]{2}{*}{$(1,0,-2,1)$} & \multirow[t]{2}{*}{4} & $(+1,0,-2,1)$ & 2 \\
\hline & & & & $(-1,0,-2,1)$ & 2 \\
\hline & & \multirow[t]{2}{*}{$(1,0,0,1)$} & \multirow[t]{2}{*}{4} & $(+1,0,0,1)$ & 2 \\
\hline & & & & $(-1,0,0,1)$ & 2 \\
\hline \multirow[t]{4}{*}{$(1,0,1,2)$} & \multirow[t]{4}{*}{12} & \multirow[t]{2}{*}{$(1,0,+1,2)$} & \multirow[t]{2}{*}{6} & $(+1,0,+1,2)$ & 3 \\
\hline & & & & $(-1,0,+1,2)$ & 3 \\
\hline & & \multirow[t]{2}{*}{$(1,0,-1,2)$} & \multirow[t]{2}{*}{6} & $(+1,0,-1,2)$ & $\overline{3}$ \\
\hline & & & & $(-1,0,-1,2)$ & 3 \\
\hline \multirow[t]{4}{*}{$(1,0,1,0)$} & \multirow[t]{4}{*}{4} & \multirow[t]{2}{*}{$(1,0,+1,0)$} & \multirow[t]{2}{*}{2} & $(+1,0,+1,0)$ & 1 \\
\hline & & & & $(-1,0,+1,0)$ & 1 \\
\hline & & \multirow[t]{2}{*}{$(1,0,-1,0)$} & \multirow[t]{2}{*}{2} & $(+1,0,-1,0)$ & 1 \\
\hline & & & & $(-1,0,-1,0)$ & 1 \\
\hline \multirow[t]{2}{*}{$(1,0,0,1)$} & \multirow[t]{2}{*}{4} & \multirow[t]{2}{*}{$(1,0,0,1)$} & \multirow[t]{2}{*}{4} & $(+1,0,0,1)$ & 2 \\
\hline & & & & $(-1,0,0,1)$ & 2 \\
\hline \multicolumn{2}{|l|}{8 subespaços } & \multicolumn{2}{|c|}{16 subespaços } & \multicolumn{2}{|c|}{24 subespaços } \\
\hline $\mathrm{d}_{3}=48$ & & $\mathrm{~d}_{3}=2$ & & $\mathrm{~d}_{3}=2$ & \\
\hline
\end{tabular}

Tabela 18b: Quebra da cadeia 9 em $L_{3, z}$ e depois em $L_{1, z}$ (não-sobrevivente). 
A próxima cadeia sobrevivente a fase 1 submetida à fase 2 é a cadeia 10 listada na tabela 19.

\begin{tabular}{|c|c|c|c|c|c|}
\hline$D_{7}$ & $A_{1} \oplus A_{1} \oplus D_{5}$ & $\mathrm{~A}_{1} \oplus \mathrm{A}_{1} \oplus \mathrm{A}_{4}$ & $\mathrm{~A}_{1} \oplus \mathrm{A}_{1} \oplus \mathrm{C}_{2}$ & $\mathrm{~A}_{1} \oplus \mathrm{A}_{1} \oplus \mathrm{A}_{1} \oplus \mathrm{A}_{1}$ & d \\
\hline \multirow{12}{*}{$(0000001 ; 64)$} & \multirow[t]{6}{*}{$(0,1,00010 ; 32)$} & \multirow{2}{*}{$(0,1,1000 ; 10)$} & \multirow[t]{2}{*}{$(0,1,01 ; 10)$} & $(0,1,1,1)$ & 8 \\
\hline & & & & $(0,1,0,0)$ & 2 \\
\hline & & \multirow[t]{3}{*}{$(0,1,0010 ; 20)$} & \multirow[t]{3}{*}{$(0,1,20 ; 20)$} & $(0,1,2,0)$ & 6 \\
\hline & & & & $(0,1,1,1)$ & 8 \\
\hline & & & & $(0,1,0,2)$ & 6 \\
\hline & & $(0,1,0000 ; 2)$ & $(0,1,00 ; 2)$ & $(0,1,0,0)$ & 2 \\
\hline & \multirow[t]{6}{*}{$(1,0,00001 ; 32)$} & \multirow[t]{2}{*}{$(1,0,0001 ; 10)$} & \multirow[t]{2}{*}{$(1,0,01 ; 10)$} & $(1,0,1,1)$ & 8 \\
\hline & & & & $(1,0,0,0)$ & 2 \\
\hline & & \multirow[t]{3}{*}{$(1,0,0100 ; 20)$} & \multirow[t]{3}{*}{$(1,0,20 ; 20)$} & $(1,0,2,0)$ & 6 \\
\hline & & & & $(1,0,1,1)$ & 8 \\
\hline & & & & $(1,0,0,2)$ & 8 \\
\hline & & $(1,0,0000 ; 2)$ & $(1,0,00 ; 2)$ & $(1,0,0,0)$ & 2 \\
\hline
\end{tabular}

Tabela 19: Cadeia 10: $D_{7} \supset A_{1} \oplus A_{1} \oplus D_{5} \supset A_{1} \oplus A_{1} \oplus A_{4} \supset A_{1} \oplus A_{1} \oplus C_{2} \supset A_{1} \oplus A_{1} \oplus A_{1} \oplus A_{1}$

Analisando a cadeia 10 verificamos que não há quebras com $L_{1, z}^{2}$ e $L_{2, z}^{2}$. Existe simetria sob a troca do primeiro e segundo $A_{1}$ e do terceiro e quarto $A_{1}, O$ terceiro e quarto $A_{1}$ não podem ser quebrado, pois se um deles é, o $\mathrm{d}_{3}$ já cai para 12 , no máximo. $\mathrm{O}$ primeiro e o segundo $A_{1}$ tem que ser quebrados, pois senão, permanecem octupletos. Então a única quebra possível é em $\left(L_{1, z}, L_{2 . z}\right)$, simultaneamente, que pode ser vista na tabela 19a. 


\begin{tabular}{|c|c|c|c|}
\hline $\mathrm{A}_{1} \oplus \mathrm{A}_{1} \oplus \mathrm{A}_{1} \oplus \mathrm{A}_{1}$ & d & $\left(\mathrm{L}_{1,2}, \mathrm{~L}_{2, \mathrm{z}}\right)$ & $\mathrm{d}$ \\
\hline \multirow{2}{*}{$(0,1,1,1)$} & 8 & $(0,+1,1,1)$ & 4 \\
\hline & & $(0,-1,1,1)$ & 4 \\
\hline \multirow[t]{2}{*}{$(0,1,0,0)$} & 2 & $(0,+1,0,0)$ & 1 \\
\hline & & $(0,-1,0,0)$ & 1 \\
\hline \multirow[t]{2}{*}{$(0,1,2,0)$} & 6 & $(0,+1,2,0)$ & 3 \\
\hline & & $(0,-1,2,0)$ & 3 \\
\hline \multirow[t]{2}{*}{$(0,1,1,1)$} & 8 & $(0,+1,1,1)$ & 4 \\
\hline & & $(0,-1,1,1)$ & 4 \\
\hline \multirow[t]{2}{*}{$(0,1,0,2)$} & 6 & $(0,+1,0,2)$ & 3 \\
\hline & & $(0,-1,0,2)$ & 3 \\
\hline \multirow[t]{2}{*}{$(0,1,0,0)$} & 2 & $(0,+1,0,0)$ & 1 \\
\hline & & $(0,-1,0,0)$ & 1 \\
\hline \multirow[t]{2}{*}{$(1,0,1,1)$} & 8 & $(+1,0,1,1)$ & 4 \\
\hline & & $(-1,0,1,1)$ & 4 \\
\hline \multirow[t]{2}{*}{$(1,0,0,0)$} & 2 & $(+1,0,0,0)$ & 1 \\
\hline & & $(-1,0,0,0)$ & 1 \\
\hline \multirow[t]{2}{*}{$(1,0,2,0)$} & 6 & $(+1,0,2,0)$ & 3 \\
\hline & & $(-1,0,2,0)$ & 3 \\
\hline \multirow[t]{2}{*}{$(1,0,1,1)$} & 8 & $(+1,0,1,1)$ & 4 \\
\hline & & $(-1,0,1,1)$ & 4 \\
\hline \multirow[t]{2}{*}{$(1,0,0,2)$} & 6 & $(+1,0,0,2)$ & 3 \\
\hline & & $(-1,0,0,2)$ & 3 \\
\hline \multirow[t]{2}{*}{$(1,0,0,0)$} & 2 & $(+1,0,0,0)$ & 1 \\
\hline & & $(-1,0,0,0)$ & 1 \\
\hline \multicolumn{2}{|l|}{12 subespacos } & \multicolumn{2}{|c|}{24 subespaços } \\
\hline \multicolumn{2}{|l|}{$\mathrm{d}_{3}=24$} & \multicolumn{2}{|c|}{$\mathrm{d}_{3}=24$} \\
\hline
\end{tabular}

Tabela 19a: Quebra da cadeia 10 em $L_{1, z}$ e em $L_{2, z}$, simultaneamente (não-sobrevivente).

Analisaremos agora a cadeia 11, a qual está listada na tabela 20.

\begin{tabular}{|c|c|c|c|c|c|}
\hline $\mathrm{D}_{7}$ & $A_{1} \oplus A_{1} \oplus D_{5}$ & $\mathrm{~A}_{1} \oplus \mathrm{A}_{1} \oplus \mathrm{A}_{4}$ & $A_{1} \oplus A_{1} \oplus A_{1} \oplus A_{2}$ & $\mathrm{~A}_{1} \oplus \mathrm{A}_{1} \oplus \mathrm{A}_{1} \oplus \mathrm{A}_{1}$ & d \\
\hline \multirow[t]{12}{*}{$(0000001 ; 64)$} & \multirow[t]{6}{*}{$(0,1,00010 ; 32)$} & \multirow[t]{2}{*}{$(0,1,1000 ; 10)$} & $(0,1,0,10 ; 6)$ & $(0,1,0,2)$ & 6 \\
\hline & & & $(0,1,1,00 ; 4)$ & $(0,1,1,0)$ & 4 \\
\hline & & \multirow[t]{3}{*}{$(0,1,0010 ; 20)$} & $(0,1,1,01 ; 12)$ & $(0,1,1,2)$ & 12 \\
\hline & & & $(0,1,0,10 ; 6)$ & $(0,1,0,2)$ & 6 \\
\hline & & & $(0,1,0,00 ; 2)$ & $(0,1,0,0)$ & 2 \\
\hline & & $(0,1,0000 ; 2)$ & $(0,1,0,00 ; 2)$ & $(0,1,0,0)$ & 2 \\
\hline & \multirow[t]{6}{*}{$(1,0,00001 ; 32)$} & \multirow[t]{2}{*}{$(1,0,0001 ; 10)$} & $(1,0,0,01 ; 6)$ & $(1,0,0,2)$ & 6 \\
\hline & & & $(1,0,1,00 ; 4)$ & $(1,0,1,0)$ & 4 \\
\hline & & \multirow[t]{3}{*}{$(1,0,0100 ; 20)$} & $(1,0,1,10 ; 12)$ & $(1,0,1,2)$ & 12 \\
\hline & & & $(1,0,0,01 ; 6)$ & $(1,0,0,2)$ & 6 \\
\hline & & & $(1,0,0,00 ; 2)$ & $(1,0,0,0)$ & 2 \\
\hline & & $(1,0,0000 ; 2)$ & $(1,0,0,00 ; 2)$ & $(1,0,0,0)$ & 2 \\
\hline
\end{tabular}

Tabela 20: Cadeia 11: $D_{7} \supset A_{1} \oplus A_{1} \oplus D_{5} \supset A_{1} \oplus A_{1} \oplus A_{4} \supset A_{1} \oplus A_{1} \oplus A_{1} \oplus A_{2} \supset A_{1} \oplus A_{1} \oplus A_{1} \oplus A_{1}$. 
Analisando a cadeia 11, verificamos que não há quebras com $L_{1, z}^{2}$ ou $L_{2, z}^{2}$ ou $L_{3, z}^{2}$. Existe simetria sob a troca do primeiro e segundo $A_{1}$. $O$ quarto $A_{1}$ não pode ser quebrado, pois senão o $d_{3}$ cai para zero. $O$ primeiro e o segundo $A_{1}$ ou o terceiro tem que ser quebrados, pois senão, permanece pelo menos um multipleto de dimensão 12 , mas o primeiro caso gera pareamento total, então é excluído. Portanto as quebras possíveis são em $L_{3, \mathrm{z}}$ e $\left(L_{1, \mathrm{z}}, L_{3, \mathrm{z}}\right)$, as quais podem serem vistas na tabela 20 a.

\begin{tabular}{|c|c|c|c|}
\hline $\mathrm{A}_{1} \oplus \mathrm{A}_{1} \oplus \mathrm{A}_{1} \oplus \mathrm{A}_{1}$ & $\mathrm{~d}$ & $L_{3, \mathrm{z}}$ & $\mathrm{d}$ \\
\hline$(0,1,0,2)$ & 6 & $(0,1,0,2)$ & 6 \\
\hline$(0,1,1,0)$ & 4 & $(0,1,+1,0)$ & 2 \\
\hline & & $(0,1,-1,0)$ & 2 \\
\hline$(0,1,1,2)$ & 12 & $(0,1,+1,2)$ & 6 \\
\hline & & $(0,1,-1,2)$ & 6 \\
\hline$(0,1,0,2)$ & 6 & $(0,1,0,2)$ & 6 \\
\hline$(0,1,0,0)$ & 2 & $(0,1,0,0)$ & 2 \\
\hline$(0,1,0,0)$ & 2 & $(0,1,0,0)$ & 2 \\
\hline$(1,0,0,2)$ & 6 & $(1,0,0,2)$ & 6 \\
\hline$(1,0,1,0)$ & 4 & $(1,0,+1,0)$ & 2 \\
\cline { 3 - 4 } & & $(1,0,-1,0)$ & 2 \\
\hline$(1,0,1,2)$ & 12 & $(1,0,+1,2)$ & 6 \\
\hline & & $(1,0,-1,2)$ & 6 \\
\hline$(1,0,0,2)$ & 6 & $(1,0,0,2)$ & 6 \\
\hline$(1,0,0,0)$ & 2 & $(1,0,0,0)$ & 2 \\
\hline$(1,0,0,0)$ & 2 & $(1,0,0,0)$ & 2 \\
\hline 12 subespaços & & 16 subespacos \\
\hline d $3=48$ & & $d_{3}=48$ \\
\hline
\end{tabular}

\begin{tabular}{|c|c|c|c|}
\hline$A_{1} \oplus A_{1} \oplus A_{1} \oplus A_{1}$ & $\mathrm{~d}$ & $\left(L_{1 . z}, L_{3 . z}\right)$ & $\mathrm{d}$ \\
\hline$(0,1,0,2)$ & 6 & $(0,1,0,2)$ & 6 \\
\hline \multirow[t]{2}{*}{$(0,1,1,0)$} & \multirow[t]{2}{*}{4} & $(0,1,+1,0)$ & 2 \\
\hline & & $(0,1,-1,0)$ & 2 \\
\hline \multirow[t]{2}{*}{$(0,1,1,2)$} & \multirow[t]{2}{*}{12} & $(0,1,+1,2)$ & 6 \\
\hline & & $(0,1,-1,2)$ & 6 \\
\hline$(0,1,0,2)$ & 6 & $(0,1,0,2)$ & 6 \\
\hline$(0,1,0,0)$ & 2 & $(0,1,0,0)$ & 2 \\
\hline$(0,1,0,0)$ & 2 & $(0,1,0,0)$ & 2 \\
\hline \multirow[t]{2}{*}{$(1,0,0,2)$} & \multirow[t]{2}{*}{6} & $(+1,0,0,2)$ & 3 \\
\hline & & $(-1,0,0,2)$ & 3 \\
\hline \multirow[t]{4}{*}{$(1,0,1,0)$} & \multirow[t]{4}{*}{4} & $(+1,0,+1,0)$ & 1 \\
\hline & & $(-1,0,+1,0)$ & 1 \\
\hline & & $(+1,0,-1,0)$ & 1 \\
\hline & & $(-1,0,-1,0)$ & 1 \\
\hline \multirow[t]{4}{*}{$(1,0,1,2)$} & \multirow[t]{4}{*}{12} & $(1,0,+1,2)$ & 3 \\
\hline & & $(1,0,+1,2)$ & 3 \\
\hline & & $(1,0,-1,2)$ & 3 \\
\hline & & $(1,0,-1,2)$ & 3 \\
\hline \multirow[t]{2}{*}{$(1,0,0,2)$} & \multirow[t]{2}{*}{6} & $(+1,0,0,2)$ & 3 \\
\hline & & $(-1,0,0,2)$ & 3 \\
\hline \multirow[t]{2}{*}{$(1,0,0,0)$} & \multirow[t]{2}{*}{2} & $(+1,0,0,0)$ & 1 \\
\hline & & $(-1,0,0,0)$ & 1 \\
\hline \multirow[t]{2}{*}{$(1,0,0,0)$} & \multirow[t]{2}{*}{2} & $(1,0,0,0)$ & 1 \\
\hline & & $(1,0,0,0)$ & 1 \\
\hline \multicolumn{2}{|l|}{12 subespaços } & \multicolumn{2}{|c|}{24 subespaços } \\
\hline \multicolumn{2}{|l|}{$\mathrm{d}_{3}=48$} & \multicolumn{2}{|l|}{$\mathrm{d}_{3}=48$} \\
\hline
\end{tabular}

Tabela 20a: Quebra da cadeia 11 em $L_{3, z}$ e $L_{3, z}$ e $L_{1,2}$ simultaneamente (não-sobrevivente).

Analisaremos agora a cadeia 12, a qual está listada na tabela 21. 


\begin{tabular}{|c|c|c|c|c|c|c|}
\hline $\mathrm{D}_{7}$ & $\mathrm{~A}_{3} \oplus \mathrm{D}_{4}$ & $\mathrm{~A}_{3} \oplus \mathrm{A}_{1} \oplus \mathrm{C}_{2}$ & $\mathrm{~A}_{3} \oplus \mathrm{A}_{1} \oplus \mathrm{A}_{1} \oplus \mathrm{A}_{1}$ & $A_{2} \oplus A_{1} \oplus A_{1} \oplus A_{1}$ & $\mathrm{~A}_{1} \oplus \mathrm{A}_{1} \oplus \mathrm{A}_{1} \oplus \mathrm{A}_{1}$ & d \\
\hline \multirow[t]{10}{*}{$(0000001 ; 64)$} & \multirow[t]{6}{*}{$(001,0010 ; 32)$} & \multirow[t]{4}{*}{$(001,0,01 ; 20)$} & \multirow[t]{2}{*}{$(001,0,1,1 ; 16)$} & $(01,0,1,1 ; 12)$ & $(2,0,1,1)$ & 12 \\
\hline & & & & $(00,0,1,1 ; 4)$ & $(0,0,1,1)$ & 4 \\
\hline & & & \multirow[t]{2}{*}{$(001,0,0,0 ; 4)$} & $(01,0,0,0 ; 3)$ & $(2,0,0,0)$ & 3 \\
\hline & & & & $(00,0,0,0 ; 1)$ & $(0,0,0,0)$ & 1 \\
\hline & & \multirow[t]{2}{*}{$(001,2,00 ; 12)$} & \multirow[t]{2}{*}{$(001,2,0,0 ; 12)$} & $(01,2,0,0 ; 9)$ & $(2,2,0,0)$ & 9 \\
\hline & & & & $(00,2,0,0 ; 3)$ & $(0,2,0,0)$ & 3 \\
\hline & \multirow[t]{4}{*}{$(100,0001 ; 32)$} & \multirow[t]{4}{*}{$(100,1,10 ; 32)$} & \multirow[t]{2}{*}{$(100,1,1,0 ; 16)$} & $(10,1,1,0 ; 12)$ & $(2,1,1,0)$ & 12 \\
\hline & & & & $(00,1,1,0 ; 4)$ & $(0,1,1,0)$ & 4 \\
\hline & & & \multirow[t]{2}{*}{$(100,1,0,1 ; 16)$} & $(10,1,0,1 ; 12)$ & $(2,1,0,1)$ & 12 \\
\hline & & & & $(00,1,0,1 ; 4)$ & $(0,1,0,1)$ & 4 \\
\hline
\end{tabular}

Tabela 21: Cadeia 12: $D_{7} \supset A_{3} \oplus D_{4} \supset A_{3} \oplus A_{1} \oplus C_{2} \supset A_{3} \oplus A_{1} \oplus A_{1} \oplus A_{1}$ $\supset A_{2} \oplus A_{1} \oplus A_{1} \oplus A_{1} \supset A_{1} \oplus A_{1} \oplus A_{1} \oplus A_{1}$.

Analisando a cadeia 12 verificamos que não há quebras com $L_{3, z}^{2}$ ou $L_{4, z}^{2}$. Existe simetria sob a troca do terceiro e quarto $A_{1}$. $O$ primeiro $A_{1}$ não pode ser quebrado, pois se é, $d_{3}$ cai para, no máximo, 12. O segundo $A_{1}$ tem que ser quebrado, já que o primeiro não é, para que o multipleto de dimensão 9 seja eliminado, e tem que ser quebrado em $L_{2, z}^{2}$, para não produzir um número excessivo de multipletos de dimensão impar (4 tripletos no total). $O$ terceiro e o quarto $A_{1}$ tem que ser quebrados para que os últimos dois multipletos de dimensão 12 sejam eliminados. Mas então o primeiro multipleto de dimensão 12 produz um número excessivo de multipletos de dimensão impar ( 6 tripletos, no total). Portanto podemos observar que nenhuma quebra sobrevive.

A próxima cadeia a ser analisada na fase 2 é a cadeia 13 , listada na tabela 22.

\begin{tabular}{|c|c|c|c|c|c|c|}
\hline $\mathrm{D}_{7}$ & $\mathrm{~B}_{3} \oplus \mathrm{B}_{3}$ & $\mathrm{~B}_{3} \oplus \mathrm{G}_{2}$ & $\mathrm{G}_{2} \oplus \mathrm{G}_{2}$ & $\mathrm{G}_{2} \oplus \mathrm{A}_{1} \oplus \mathrm{A}_{1}$ & $\mathrm{~A}_{1} \oplus \mathrm{A}_{1} \oplus \mathrm{A}_{1} \oplus \mathrm{A}_{1}$ & d \\
\hline \multirow[t]{9}{*}{$(0000001 ; 64)$} & \multirow[t]{9}{*}{$(001,001 ; 64)$} & \multirow[t]{6}{*}{$(001,01 ; 56)$} & \multirow[t]{4}{*}{$(01,01 ; 49)$} & \multirow[t]{2}{*}{$(01,1,1 ; 28)$} & $(1,1,1,1)$ & 16 \\
\hline & & & & & $(0,2,1,1)$ & 12 \\
\hline & & & & \multirow[t]{2}{*}{$(01,0,2 ; 21)$} & $(1,1,0,2)$ & 12 \\
\hline & & & & & $(0,2,0,2)$ & 9 \\
\hline & & & \multirow[t]{2}{*}{$(00,01 ; 7)$} & $(00,1,1 ; 4)$ & $(0,0,1,1)$ & 4 \\
\hline & & & & $(00,0,2 ; 3)$ & $(0,0,0,2)$ & 3 \\
\hline & & \multirow[t]{3}{*}{$(001,00 ; 8)$} & \multirow[t]{2}{*}{$(01,00 ; 7)$} & \multirow[t]{2}{*}{$(01,0,0 ; 7)$} & $(1,1,0,0)$ & 4 \\
\hline & & & & & $(0,2,0,0)$ & 3 \\
\hline & & & $(00,00 ; 1)$ & $(00,0,0 ; 1)$ & $(0,0,0,0)$ & 1 \\
\hline
\end{tabular}

Tabela 22: Cadeia 13: $D_{7} \supset B_{3} \oplus B_{3} \supset B_{3} \oplus G_{2} \supset G_{2} \oplus G_{2} \supset G_{2} \oplus A_{1} \oplus A_{1} \supset A_{1} \oplus A_{1} \oplus A_{1} \oplus A_{1}$. 
Analisando a cadeia 13, verificamos que não há quebras em $L_{l, z}^{2}$ ou $L_{3, z}^{2}$. Existe simetria sob a troca do primeiro mais o segundo $A_{1}$ com o terceiro mais o quarto $A_{1}$. O segundo $A_{1}$ tem que ser quebrado em $L_{2, z}^{2}$, para não produzir um número excessivo de multipletos de dimensão impar (4 tripletos mais 4 singletos, no total). O primeiro e o terceiro $A_{1}$ tem que ser quebrados para que o multipleto de dimensão 16 seja eliminado. $O$ quarto $A_{1}$ tem que ser quebrado em $L_{4, z}^{2}$, para não produzir um número excessivo de multipletos de dimensão 1 , mas quando ele é quebrado primeiro em $L_{4 . z}^{2}$, em qualquer outra quebra posterior o $d_{3}$ cai para zero. Portanto a única quebra possível é $\left(L_{1, z}, L_{2, z}^{2}, L_{3, z}\right)$, a qual pode ser vista na tabela 22a. Podemos ressaltar, que essa cadeia chega bem perto do código genético.

\begin{tabular}{|c|c|c|c|}
\hline $\mathrm{A}_{1} \oplus \mathrm{A}_{1} \oplus \mathrm{A}_{1} \oplus \mathrm{A}_{1}$ & $\mathrm{~d}$ & $\left(L_{1, z}, L_{2, z}^{2}, L_{3, z}\right)$ & $d$ \\
\hline \multirow[t]{4}{*}{$(1,1,1,1)$} & \multirow[t]{4}{*}{16} & $(+1, \pm 1,+1,1)$ & 4 \\
\hline & & $(+1, \pm 1,-1,1)$ & 4 \\
\hline & & $(-1, \pm 1,+1,1)$ & 4 \\
\hline & & $(-1, \pm 1,-1,1)$ & 4 \\
\hline \multirow[t]{4}{*}{$(0,2,1,1)$} & \multirow[t]{4}{*}{12} & $(0, \pm 2,+1,1)$ & 4 \\
\hline & & $(0, \pm 2,-1,1)$ & 4 \\
\hline & & $(0,0,1,+1)$ & 2 \\
\hline & & $(0,0,1,-1)$ & 2 \\
\hline \multirow[t]{2}{*}{$(1,1,0,2)$} & \multirow[t]{2}{*}{12} & $(+1,1,0,2)$ & 6 \\
\hline & & $(-1,1,0,2)$ & 6 \\
\hline \multirow[t]{2}{*}{$(0,2,0,2)$} & \multirow[t]{2}{*}{9} & $(0, \pm 2,0,2)$ & 6 \\
\hline & & $(0,0,0,2)$ & 3 \\
\hline \multirow[t]{2}{*}{$(0,0,1,1)$} & \multirow[t]{2}{*}{4} & $(0,0,+1,1)$ & 2 \\
\hline & & $(0,0,-1,1)$ & 2 \\
\hline$(0,0,0,2)$ & 3 & $(0,0,0,2)$ & 3 \\
\hline \multirow[t]{2}{*}{$(1,1,0,0)$} & \multirow[t]{2}{*}{4} & $(+1, \pm 1,0,0)$ & 2 \\
\hline & & $(+1, \pm 1,0,0)$ & 2 \\
\hline \multirow[t]{2}{*}{$(0,2,0,0)$} & \multirow[t]{2}{*}{3} & $(0, \pm 2,0,0)$ & 2 \\
\hline & & $(0,0,0,0)$ & 1 \\
\hline$(0,0,0,0)$ & 1 & $(0,0,0,0)$ & 1 \\
\hline \multicolumn{2}{|l|}{9 subespaços } & \multicolumn{2}{|c|}{20 subespaços } \\
\hline \multicolumn{2}{|c|}{$\mathrm{d}_{3}=39$} & \multicolumn{2}{|c|}{$\mathrm{d}_{3}=24$} \\
\hline
\end{tabular}

Tabela 22a: Quebra da cadeia $13 \mathrm{em}\left(L_{1, \mathrm{z}}, L_{2, \mathrm{z}}^{2}, L_{3, \mathrm{z}}\right)$ (não-sobrevivente).

Analisaremos agora a cadeia 14, a qual está listada na tabela 23. 


\begin{tabular}{|c|c|c|c|c|c|}
\hline $\mathrm{D}_{7}$ & $\mathrm{~B}_{3} \oplus \mathrm{B}_{3}$ & $B_{3} \oplus A_{1} \oplus A_{1} \oplus A_{1}$ & $G_{2} \oplus A_{1} \oplus A_{1} \oplus A_{1}$ & $\mathbf{A}_{1} \oplus \mathbf{A}_{1} \oplus \mathbf{A}_{1} \oplus \mathbf{A}_{1} \oplus \mathbf{A}_{1}$ & d \\
\hline \multirow{6}{*}{$(0000001 ; 64)$} & \multirow[t]{6}{*}{$(001,001 ; 64)$} & \multirow[t]{3}{*}{$(001,1,0,1 ; 32)$} & \multirow[t]{2}{*}{$(01,1,0,1 ; 28)$} & $(1,1,1,0,1)$ & 16 \\
\hline & & & & $(0,2,1,0,1)$ & 12 \\
\hline & & & $(00,1,0,1 ; 4)$ & $(0,0,1,0,1)$ & 4 \\
\hline & & \multirow[t]{3}{*}{$(001,0,1,1 ; 32)$} & \multirow[t]{2}{*}{$(01,0,1,1 ; 28)$} & $(1,1,0,1,1)$ & 16 \\
\hline & & & & $(0,2,0,1,1)$ & 12 \\
\hline & & & $(00,0,1,1 ; 4)$ & $(0,0,0,1,1)$ & 4 \\
\hline
\end{tabular}

Tabela 23: Cadeia 14: $D_{7} \supset B_{3} \oplus B_{3} \supset B_{3} \oplus A_{1} \oplus A_{1} \oplus A_{1} \supset G_{2} \oplus A_{1} \oplus A_{1} \oplus A_{1}$ $\supset A_{1} \oplus A_{1} \oplus A_{1} \oplus A_{1} \oplus A_{1}$.

Analisando a cadeia 14 , verificamos que não há quebras com $L_{1, z}^{2}, L_{3, z}^{2}, L_{4, z}^{2}$ ou $L_{5, z}^{2}$. Existe simetria sob a troca do terceiro e quarto $A_{1}$. O segundo $A_{1}$ não pode ser quebrado, pois nas duas quebras, $L_{4, z}^{2}$ e $L_{4, z}, \circ d_{3}$ cai para zero. $O$ quinto $A_{1}$ tem que ser quebrado, pois senão, todas as dimensões seriam multipletos de 2 (pares). $O$ terceiro e o quarto $A_{1}$ não podem ser quebrados, para evitar que apareçam 4 ou 8 singletos. $O$ primeiro $A_{1}$ tem que ser quebrado já que o segundo, terceiro e quarto não, para que o multipleto de dimensão 16 seja eliminado. Portanto só é possível a quebra em $\left(L_{1, z}, L_{5,2}\right)$, a qual apresenta somente sextupletos, dubletos e quartetos (tabela 23a).

\begin{tabular}{|c|c|c|c|c|c|}
\hline $\mathbf{A}_{1} \oplus \mathbf{A}_{1} \oplus \mathbf{A}_{1} \oplus \mathbf{A}_{1} \oplus \mathbf{A}_{1}$ & $\mathrm{~d}$ & $L_{\mathrm{I}, \mathrm{z}}$ & d & $\left(L_{1, \mathrm{z}}, L_{S . \mathrm{z}}\right)$ & d \\
\hline \multirow[t]{4}{*}{$(1,1,1,0,1)$} & \multirow[t]{4}{*}{16} & \multirow[t]{2}{*}{$(+1,1,1,0,1)$} & \multirow[t]{2}{*}{8} & $(+1,1,1,0,+1)$ & 4 \\
\hline & & & & $(+1,1,1,0,-1)$ & 4 \\
\hline & & \multirow[t]{2}{*}{$(-1,1,1,0,1)$} & \multirow[t]{2}{*}{8} & $(-1,1,1,0,+1)$ & 4 \\
\hline & & & & $(-1,1,1,0,-1)$ & 4 \\
\hline \multirow[t]{2}{*}{$(0,2,1,0,1)$} & \multirow[t]{2}{*}{12} & \multirow[t]{2}{*}{$(0,2,1,0,1)$} & \multirow[t]{2}{*}{12} & $(0,2,1,0,+1)$ & 6 \\
\hline & & & & $(0,2,1,0,-1)$ & 6 \\
\hline \multirow[t]{2}{*}{$(0,0,1,0,1)$} & \multirow[t]{2}{*}{4} & \multirow[t]{2}{*}{$(0,0,1,0,1)$} & \multirow[t]{2}{*}{4} & $(0,0,1,0,+1)$ & 2 \\
\hline & & & & $(0,0,1,0,-1)$ & 2 \\
\hline \multirow[t]{4}{*}{$(1,1,0,1,1)$} & \multirow[t]{4}{*}{16} & \multirow[t]{2}{*}{$(+1,1,0,1,1)$} & \multirow[t]{2}{*}{8} & $(+1,1,0,1,+1)$ & 4 \\
\hline & & & & $(+1,1,0,1-1)$ & 4 \\
\hline & & \multirow[t]{2}{*}{$(-1,1,0,1,1)$} & \multirow[t]{2}{*}{8} & $(-1,1,0,1,+1)$ & 4 \\
\hline & & & & $(-1,1,0,1,-1)$ & 4 \\
\hline \multirow[t]{2}{*}{$(0,2,0,1,1)$} & \multirow[t]{2}{*}{12} & \multirow[t]{2}{*}{$(0,2,0,1,1)$} & \multirow[t]{2}{*}{12} & $(0,2,0,1,+1)$ & 6 \\
\hline & & & & $(0,2,0,1,-1)$ & 6 \\
\hline \multirow[t]{2}{*}{$(0,0,0,1,1)$} & \multirow[t]{2}{*}{4} & \multirow[t]{2}{*}{$(0,0,0,1,1)$} & \multirow[t]{2}{*}{4} & $(0,0,0,1,+1)$ & $\frac{\sqrt{2}}{2}$ \\
\hline & & & & $(0,0,0,1,-1)$ & 2 \\
\hline \multicolumn{2}{|l|}{6 subespaços } & \multicolumn{2}{|c|}{8 subespaços } & \multicolumn{2}{|c|}{16 subespacos } \\
\hline \multicolumn{2}{|l|}{$d_{3}=24$} & \multicolumn{2}{|c|}{$d_{3}=24$} & \multicolumn{2}{|c|}{$\mathrm{d}_{3}=24$} \\
\hline
\end{tabular}

Tabela 23a: Quebra da cadeia 14 em $L_{1, z}$ e depois em $L_{5, z}$ (não-sobrevivente). 
Analisaremos agora a última cadeia sobrevivente a fase 1 , a cadeia 15 , a qual é mostrada na tabela 24 .

\begin{tabular}{c|c|c|c|c|c|}
\hline $\mathrm{D}_{7}$ & $\mathrm{~A}_{3} \oplus \mathrm{D}_{4}$ & $\mathrm{~A}_{3} \oplus \mathrm{A}_{1} \oplus \mathrm{A}_{1} \oplus \mathrm{A}_{1} \oplus \mathrm{A}_{1}$ & $\mathrm{~A}_{2} \oplus \mathrm{A}_{1} \oplus \mathrm{A}_{1} \oplus \mathrm{A}_{1} \oplus \mathrm{A}_{1}$ & $\mathrm{~A}_{1} \oplus \mathrm{A}_{1} \oplus \mathrm{A}_{1} \oplus \mathrm{A}_{1} \oplus \mathrm{A}_{1}$ & $\mathrm{~d}$ \\
\hline$(0000001 ; 64)$ & $(001,0010 ; 32)$ & $(001,0,1,1,0 ; 16)$ & $(01,0,1,1,0 ; 12)$ & $(2,0,1,1,0)$ & 12 \\
\hline & & & $(00,0,1,1,0 ; 4)$ & $(0,0,1,1,0)$ & 4 \\
\hline & & $(001,1,0,0,1 ; 16)$ & $(01,1,0,0,1 ; 12)$ & $(2,1,0,0,1)$ & 12 \\
\hline & & $(00,1,0,0,1 ; 4)$ & $(0,1,0,0,1)$ & 4 \\
\hline & & & $(10,0,1,0,1 ; 12)$ & $(2,0,1,0,1)$ & 12 \\
\hline & & & $(00,0,1,0,1 ; 4)$ & $(0,0,1,0,1)$ & 4 \\
\hline & & & $(10,1,0,0,1 ; 12)$ & $(2,1,0,0,1)$ & 12 \\
\hline & & $(100,1,0,0,1 ; 16)$ & $(00,1,0,0,1 ; 4)$ & $(0,1,0,0,1)$ & 4 \\
\hline
\end{tabular}

Tabela 24 : Cadeia $15: \mathrm{D}_{7} \supset \mathrm{A}_{3} \oplus \mathrm{D}_{4} \supset \mathrm{A}_{3} \oplus \mathrm{A}_{1} \oplus \mathrm{A}_{1} \oplus \mathrm{A}_{1} \oplus \mathrm{A}_{1} \supset \mathrm{A}_{2} \oplus \mathrm{A}_{1} \oplus \mathrm{A}_{1} \oplus \mathrm{A}_{1} \oplus \mathrm{A}_{1}$ $\supset \mathrm{A}_{1} \oplus \mathrm{A}_{1} \oplus \mathrm{A}_{1} \oplus \mathrm{A}_{1} \oplus \mathrm{A}_{1}$

Analisando a cadeia acima, verificamos que não há quebras em $L_{2.2}^{2}, L_{3.2}^{2}, L_{4.2}^{2}$ ou $L_{5.2}^{2}$. Existe simetria soba troca do segundo e terceiro $A_{1}$ e do quarto e quinto $A_{1}$, assim como dos blocos segundo mais terceiro e quarto mais quinto. $\mathrm{O}$ primeiro $\mathrm{A}_{1}$ não pode ser quebrado, pois o $\mathrm{d}_{3}$ cai pra zero. A única quebra notável é quebrar em $L_{2, z}$ e $L_{3, z}$ e não em $L_{4, z}$ nem em $L_{5, z}$ : senão, permanecem multipletos de dimensão 12 ou não permanecem sextupletos. Portanto só temos a quebra $\left(L_{2, z}, L_{3, z}\right)$, a qual produz somente sextupletos e dubletos (veja tabela 24a).

\begin{tabular}{|c|c|c|c|}
\hline$A_{1} \oplus A_{1} \oplus A_{1} \oplus A_{1} \oplus A_{l}$ & d & $\left(L_{2, z}, L_{3, z}\right)$ & $\mathrm{d}$ \\
\hline \multirow[t]{2}{*}{$(2,0,1,1,0)$} & \multirow[t]{2}{*}{12} & $(2,0,+1,1,0)$ & 6 \\
\hline & & $(2,0,-1,1,0)$ & 6 \\
\hline \multirow[t]{2}{*}{$(0,0,1,1,0)$} & \multirow[t]{2}{*}{4} & $(0,0,+1,1,0)$ & 2 \\
\hline & & $(0,0,-1,1,0)$ & 2 \\
\hline \multirow[t]{2}{*}{$(2,1,0,0,1)$} & \multirow[t]{2}{*}{12} & $(2,+1,0,0,1)$ & 6 \\
\hline & & $(2,-1,0,0,1)$ & 6 \\
\hline \multirow[t]{2}{*}{$(0,1,0,0,1)$} & \multirow[t]{2}{*}{4} & $(0,+1,0,0,1)$ & 2 \\
\hline & & $(0,-1,0,0,1)$ & 2 \\
\hline \multirow[t]{2}{*}{$(2,0,1,0,1)$} & \multirow[t]{2}{*}{12} & $(2,0,+1,0,1)$ & 6 \\
\hline & & $(2,0,-1,0,1)$ & 6 \\
\hline \multirow[t]{2}{*}{$(0,0,1,0,1)$} & \multirow[t]{2}{*}{4} & $(0,0,+1,0,1)$ & 2 \\
\hline & & $(0,0,-1,0,1)$ & 2 \\
\hline \multirow[t]{2}{*}{$(2,1,0,0,1)$} & \multirow[t]{2}{*}{12} & $(2,+1,0,0,1)$ & 6 \\
\hline & & $(2,-1,0,0,1)$ & 6 \\
\hline \multirow[t]{2}{*}{$(0,1,0,0,1)$} & \multirow[t]{2}{*}{4} & $(0,+1,0,0,1)$ & 2 \\
\hline & & $(0,-1,0,0,1)$ & 2 \\
\hline \multicolumn{2}{|l|}{8 subespaços } & \multicolumn{2}{|c|}{16 subespaços } \\
\hline \multicolumn{2}{|l|}{$\mathrm{d}_{3}=48$} & \multicolumn{2}{|c|}{$\mathrm{d}_{3}=48$} \\
\hline
\end{tabular}

Tabela 24a: Quebra da cadeia 15 em $\left(L_{2, z}, L_{3, z}\right)$ (não-sobrevivente). 
Com isso terminamos a análise na fase 2 das cadeias das álgebras $B_{6}$ e $D_{7}$ e concluímos que não existe outras álgebras alem do $G_{2}$ e do $C_{3}(s p(6))$ que reproduzem a degenerescência do código genético. 


\section{Capítulo 6}

\section{Conclusões}

Nessa dissertação foram elaborados critérios baseados nas características das álgebras de Lie, que permitiram a redução de centenas de cadeias de álgebras possíveis a pouco mais de uma dezena. As noções de cadeias sobreviventes e emparelhamento dirnensinal, possibilitaram com rigor a análise da degenerescência de uma álgebra de Lie nurna determinada cadeia de subálgebras maximais.

Após analisar centenas de cadeias, usando critérios de eliminação, verificamos que apenas 13 cadeias foram classificadas como sobrevivente na fase 1 . Dentre estas 13 cadeias, as quais foram submetidas à fase 2 , sornente as álgebras $C_{3}(\operatorname{sp}(6))$ e $G_{2}$ apresentaram cadeias que reproduzern a degenerescência do código genético, isto é, se houver urm congelamento apropriado, confirmando assim, os resultados de Hornos e Hormos, e Forger et all ${ }^{23}$. Dessa forma concluímos a procura por simetrias no código genético padrão e abrimos caminho para o estudo de códigos excepcionais característico das mitocondrias. 


\section{Capítulo 7}

\section{Bibliografia}

[1] M. Hamermesh Group Theory an its Applications to Physical Problems. Addisn-Wesley, 1962.

[2] B. G. Wybourne. Classical Groups for Physicists. John Wiley, 1974.

[3] A. O. Barut and R. Raczka.Theory of Group Representations and Applications. World Scientific, 1986.

[4] D. P. Zelobenco. Compact Lie Groups and their Representations volume 40 of Translations of Mathematical Monographs. AMS, 1978.

[5] R. Gilmore. Lie Groups, Lie Algebras and same of their Applications. John Wiley, 1974.

[6] Jin-Quan Chen. Group Representation Theory for Physicists. World Sientific, 1989.

[7] L. Stryer. Bioquimica, 1988.

[8] J. E. M. Hornos and Y. M. M. Hornos. Algebraic Model for the Evolution of the Genetic Code. Physical Review Letters vol. 71, N. 26.

[9] J. Maddox. The Genetic Code by Numbers. Nature vol. 367.

[10] W. G. Mckay and J. Patera. Tables of Dimennsions, Indices and Branching Rules For Representations of Simple Lie Algebras, Lecture Notes in Pure and Applied Mathematics. Marcel Dikker, vol.69, New York, 1968.

[11] J.D. Watson and F.H. Crick:, Nature 131 (1953).

[12]F.H. Crick, L. Barnett, S. Brenner and R.J. Watts-Tobin: General Nature of the Genetic Code for Proteins, Nature 192 (1961).

[13]M.W. Nirenberg and J.H. Matthaẹi, Proc.Natl.Acad.Sci. USA 47 (1961).

[14]P. Lengyl, J.F. Speyer and S.Ochoa, Proc.Natl.Acad.Sci. USA 47 (1961). 
[15] M.W. Nirenberg, Sci.Am. 208, Vol. 3 (1963)

[16] M.W. Nirenberg and P. Leder, Science 145 (1964).

[17] M. Hausner and J. T. Schwartz, Lie Groups; Lie Algebras, Gordon and Breach, New York, 1968.

[18] E. P. Wigner, Gruppenntheorie, viewig, Brunswick, Germany, 1931; expanded English transl. By J. J. Griffin, Group Theory, Academic Press, New York, 1959.

[19] G. Raacah, "Group Theory and Spectroscopy" Ergeb. Exakt. Naturwiss., 37, 28 (1965).

[20] I. D. Ado. Lie Groups. AMS Translations vol. 3 , 1962.

[21] J. E. Humphreys, Introduction to Lie Álgebras and Representation Theory, Springer, Berlin (1972).

[22] J. E. M. Hornos and Y. M. M. Hornos, A Search for Symmetries in the Genetic Code, J. Biol. Phys. 20 (1994).

[23] J. E. M. Hornos , Y. M. M. Hornos and M. Forger, Symmetry and Symmetry Breaking: An Algebraic Approach to the Genetic Code.

[24] S. Ozawa, T.H. Jukes, K. Watanabe and A. Muto: Recent Evidence for the Evolution of the Genetic Code, Microbiol. Rev. 56 (1992) 229-264.

[25] F.H.C. Crick: The Origen of the Genetic Code, J. Mol. Biol. 38 (1968) 367-379.

[26] M. O. Bertman and Jr. Jungck: Some Unresolved Mathematical Problems in Genetic Coding, Not. AMS 25 (1978) A-174. 